\title{
Der Einfluß von chemischer Konstitution, Symmetrie und molekularer Umgebung auf die intramolekulare Schwingungsrelaxation aromatischer Moleküle
}

\author{
Dissertation \\ zur Erlangung des Doktorgrades \\ der Mathematisch-Naturwissenschaftlichen Fakultäten \\ der Georg-August-Universität zu Göttingen
}

vorgelegt von

Rebekka von Benten

aus Braunschweig

Göttingen 2005 
D 7

Referent: $\quad$ Prof. Dr. Jürgen Troe

Korreferent: Prof. Dr. Peter Botschwina

Tag der mündlichen Prüfung: 18.01.2006 
There is a theory which states that if ever anyone discovers exactly what the Universe is for and why it is here, it will instantly disappear and be replaced by something even more bizarre and inexplicable.

There is another theory which states that this has already happened. Douglas Adams, The Hitchhiker's Guide to the Galaxy 



\section{Dank}

An erster Stelle gebührt mein Dank meinem Doktorvater, Prof. Jürgen Troe. Für die wohlwollende Betreuung meiner Arbeit in diesem kreativen Umfeld bin ich ihm zutiefst verbunden.

Die Dankbarkeit für meinen Chef, Prof. Bernd Abel, kann ich gar nicht in Worte fassen. Er hat mir alle kreativen Freiräume gelassen, die ich brauchte, und mir gleichzeitig immer mit sinnvollem Rat zur Seite gestanden. Viele Ideen zu neuen Experimenten sind uns erst bei den ausführlichen Diskussionen gekommen, für die er trotz seines vollen Terminkalenders noch jedesmal Zeit gefunden hat.

Prof. Dirk Schwarzer möchte ich ebenfalls sehr herzlich für die stete Diskussionsbereitschaft danken. Viele Fehlversuche konnten vermieden werden, weil ich von seiner Erfahrung auf dem Gebiet der Spektroskopie in überkritischen Medien profitieren konnte.

Meinen Büromitinsassen kann ich gar nicht genug danken für die gute Zeit, die wir miteinander verbracht haben. Oliver, Du hast meinen grauen Wissenschaftsalltag bereichert, und das nicht nur im Labor. Esteban, kleiner Doktorand, logg Dich doch nochmal auf dem Lizenz-Server ein... Allen anderen „Trojanern“, ganz besonders aber den übrigen „Abels“ Aleš, Andi, Björn, Evgenij, Manfred, Rosalin und Tim spreche ich ein dickes Dankeschön für ihre Kollegialität, Hilfsbereitschaft und ihren Humor aus.

Björn und Oli haben mir als Korrekturleser dieser Arbeit sehr geholfen; Dr. Jörg Hahns Texpertise war abermals von unschätzbarem Wert.

Dr. Jens Aßmann hat mich nicht nur in die Ultrakurzzeitspektroskopie eingeführt, sondern auch seine gesamte übrige Erfahrung selbstlos an mich weitergegeben. I still respect his authority.

Dem neuen Lasersystem mußten einige Kinderkrankheiten ausgetrieben werden, ehe es seinen Dienst erfüllen konnte. Für die lehrreichen Servicevisits danke ich wärmstens Herrn Hansi Hechler von Spectra Physics. 
Die Konzeption und Anfertigung der Meßzellen und des Hochdruckstandes wären nicht möglich gewesen ohne den unermüdlichen Einsatz unserer Werkstätten. Stellvertretend für alle Werkstattsangehörigen danke ich dafür an dieser Stelle besonders Herrn Meyer und Herrn Knorr vom Institut für Physikalische Chemie sowie Herrn Schmidt vom Max-Planck-Institut für biophysikalische Chemie.

So manches graues Haar vor blauem Bildschirm ist mir erspart geblieben, weil Dr. rer. comp. Rainer Oswald nie weiter als zwei Stockwerke entfernt war.

Christian Reichardt hat die heißen Cary-Spektren für mich aufgenommen und vorausgewertet, für diese Mühe möchte ich ihm herzlich danken.

Für die fleißige Hilfe bei den Messungen der „heißen Phase“ geht ein besonderer Dank an Katrin Siefermann nach Karlsruhe.

Diese Arbeit wäre niemals zustande gekommen, hätte mich nicht meine Familie mit ihrer Liebe und Unterstützung unermüdlich durch die Jahre begleitet. Mama, Papa, Friederike und Oma: Ich danke Euch aus tiefstem Herzen. 


\section{Inhaltsverzeichnis}

$\begin{array}{lr}\text { Zusammenfassung } & 1\end{array}$

1 Einleitung 3

2 Grundlagen $\quad 7$

2.1 Intramolekulare Dynamik . . . . . . . . . . . . . . 8

2.1.1 IVR im isolierten Molekül . . . . . . . . . . . . . . . . . 8

2.1 .2 Das IVR-Stufenmodell . . . . . . . . . . . . . . . . 13

2.1.3 Der Quantenzahl-Zustandsraum . . . . . . . . . . . . 14

2.1.4 Das empirische QNS-Modell . . . . . . . . . . . . . . 16

2.1.5 Simulation von IVR-Prozessen im QNS . . . . . . . . . . . 20

2.1.6 Bekannte Strukturfaktoren . . . . . . . . . . . . . . . . 21

2.2 Intermolekulare Dynamik . . . . . . . . . . . . . . . . . . . . 22

2.2.1 VET in der Gasphase . . . . . . . . . . . . . . . . . . . . 22

2.2.2 VET in dichten molekularen Umgebungen . . . . . . . . 23

2.2.3 IVR im nicht-isolierten Molekül . . . . . . . . . . . . . 24

3 Experimentelles $\quad 27$

3.1 Die IR-Pump-UV-Probe-Spektroskopie . . . . . . . . . . . . . . . 27

3.2 Die Komponenten des Lasersystems . . . . . . . . . . . . . . . . . 31

3.2.1 Das Hurricane ${ }^{\mathrm{TM}}-$ System . . . . . . . . . . . . . . . . . 32

3.2.2 Das NOPA-System: Erzeugung von UV-Pulsen . . . . . . . 40

3.2.3 Das TOPAS-System: Erzeugung von IR-Pulsen . . . . . . 45

3.2.4 Das Pump-Probe-Spektrometer . . . . . . . . . . . 48

3.2.5 Signalaufnahme und Signal-zu-Rausch-Verhältnis . . . . . 52

3.3 Realisierung weiter Druck- und Temperaturbereiche . . . . . . 55 
3.3.1 Die Hochdruck-/Vakuumapparatur . . . . . . . . . . . . 55

3.3 .2 Die Meßzellen . . . . . . . . . . . . . . . . . . 57

4 Modellsysteme $\quad 59$

4.1 Benzol . . . . . . . . . . . . . . . . . . . 62

4.2 Benzol $\mathrm{d}_{1} \ldots \ldots \ldots \ldots \ldots \ldots$

4.3 Chlorbenzol ......................... 74

4.4 Toluol . . . . . . . . . . . . . . . . . . . . . . . . 79

$4.5 \quad \alpha, \alpha, \alpha$-Trifluortoluol . . . . . . . . . . . . . . . . 85

4.6 Zustandsdichten . . . . . . . . . . . . . . . . . . 90

5 Ergebnisse $\quad 93$

5.1 Gasphasenexperimente an quasi-isolierten Molekülen . . . . . . . 94

5.1 .1 Messungen an Benzol . . . . . . . . . . . . . . . . . 94

5.1.2 Messungen an Benzol- $\mathrm{d}_{1} \ldots \ldots \ldots$. . . . . . . 103

5.1 .3 Messungen an Chlorbenzol . . . . . . . . . . . . . . 106

5.1.4 Messungen an Toluol . . . . . . . . . . . . . . . 107

5.1.5 Messungen an $\alpha, \alpha, \alpha$-Trifluortoluol . . . . . . . . . . . . 109

5.1.6 Vergleich der fünf aromatischen Modellsysteme . . . . . . 110

5.2 Experimente in unterschiedlichen molekularen Umgebungen . . . 112

5.2.1 Messungen mit Helium . . . . . . . . . . . . . . . . . . . . 113

5.2 .2 Messungen mit Argon . . . . . . . . . . . . . . . . . 113

5.2.3 Messungen mit Kohlendioxid . . . . . . . . . . . . . . . 113

5.2.4 Messungen mit 1,1,2-Trichlortrifluorethan . . . . . . . . 117

5.2.5 Messungen mit $n$-Perfluorpentan . . . . . . . . . . . 117

5.2 .6 Vergleich der Inertgasumgebungen . . . . . . . . . . . . . . 118

5.3 QNS-Simulationen . . . . . . . . . . . . . . . 121

5.3.1 Generierung der QNS-Signale . . . . . . . . . . . . 121

5.3.2 Kalibration der QNS-Parameter und Simulation experimenteller Absorptions-Zeit-Profile . . . . . . . . . . . . . 122

5.3.3 Vorhersage der IVR-Dynamik nach $2 v_{\mathrm{CH}}$-Anregung . . . . 124

5.3.4 Vorhersage der IVR-Dynamik nach $1 v_{\mathrm{CH}^{-}}$Anregung . . . . 128

6 Auswertung 135

6.1 Grundlagen der Auswertung . . . . . . . . . . . . . . . . 135

6.1.1 Interpretation der Pump-Probe-Signale . . . . . . . . . 135 
Inhaltsverzeichnis

6.1.2 Kinetische Modellierung . . . . . . . . . . . . . . . . . . 139

6.2 Resultate der Auswertung . . . . . . . . . . . . . . . . . . . . 142

6.2.1 Messungen an quasi-isolierten Aromaten . . . . . . . . . . 142

6.2.2 Messungen an Benzol-Inertgas-Mischungen . . . . . . . . . 149

7 Diskussion 155

7.1 IVR in quasi-isolierten Molekülen . . . . . . . . . . . . . 155

7.1 .1 Benzol . . . . . . . . . . . . . . . . . 155

7.1.2 Molekulare Symmetrie und IVR . . . . . . . . . . . . . 162

7.1.3 Interne Rotation und IVR . . . . . . . . . . . . . . . . 174

7.1.4 Beurteilung der relativen Substituenteneffekte . . . . . . . 177

7.2 IVR in unterschiedlichen molekularen Umgebungen . . . . . . . . 181

7.2.1 Dichteabhängigkeit des Energietransfers . . . . . . . . . . 181

7.2.2 Effekt der Stoßgaseffizienz . . . . . . . . . . . . . . . . . 184

7.2.3 Konkurrenz von Substituenten- und Umgebungseinflüssen . 188

$\begin{array}{lr}\text { Anhang } & 191\end{array}$

A.1 Eichparameter der energieabhängigen Absorption . . . . . . . . . 191

A.2 Berechnung der Zustandsdichte eines Moleküls mit internem

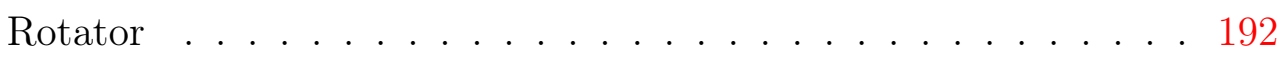

A.3 Parameter der Monte Carlo-Berechnung von g(r) für Benzol-CO ${ }_{2}$. 192

Abbildungsverzeichnis

Tabellenverzeichnis

Literaturverzeichnis 



\section{Zusammenfassung}

Diese Arbeit widmet sich der Aufklärung von Mechanismen der intramolekularen Schwingungsenergieumverteilung (IVR) im elektronischen Grundzustand mittels einer vergleichenden Studie an strukturell eng verwandten aromatischen Molekülen in unterschiedlichen molekularen Umgebungen. Aus Sicht des Chemikers ist ein Verständnis des Energietransfers in Lösung zwingend notwendig. Da hier jedoch intra- und intermolekularer Energietransfer (VET) miteinander konkurrieren, erwies es sich als vorteilhaft, zunächst quasi-isolierte Moleküle in der Gasphase zu untersuchen, wo IVR und VET auf getrennten Zeitskalen ablaufen. Überkritische Lösungsmittel boten dann die Möglichkeit, durch eine Variation der Dichte den Einfluß der Umgebung schrittweise zu verstärken.

Die Experimente wurden mit der Femtosekunden-IR-Pump-UV-Probe-Spektroskopie durchgeführt. Der Fortschritt der Energieumverteilung nach lokaler Anregung hochfrequenter CH-Streckschwingungen wurde anhand der Besetzung niederfrequenter Franck-Condon-aktiver Normalmoden der Moleküle verfolgt. Im Rahmen dieser Arbeit wurde erstmals demonstriert, daß sich diese Technik hervorragend für die Untersuchung desselben Modellsystems in unterschiedlichen Aggregatzuständen eignet.

Die Gasphasenexperimente zeigten, daß die intramolekulare Umverteilung der Anregungsenergie in quasi-isolierten Benzolderivaten einem hierarchischen Kopplungsmechanismus folgt. Der erste Schritt der Umverteilung vollzieht sich auf einer sub-ps-Zeitskala. Für den zweiten Schritt wurden Zeitkonstanten zwischen $48 \pm 5$ ps (Benzol) und $3.8 \pm 0.5$ ps $(\alpha, \alpha, \alpha$-Trifluortoluol) bestimmt. Eine Skalierung der Umverteilungsgeschwindigkeit mit der Gesamtzustandsdichte der Systeme liegt nicht vor.

Die IVR-Beeinflussung durch chemische Substitution wurde mit besonderem Schwerpunkt auf molekularer Symmetrie und interner Rotation der Benzolderivate untersucht. In einem Vergleich der Systeme Benzol und Benzol- $\mathrm{d}_{1}$ wur- 
de eine IVR-Beschleunigung um einen Faktor 3-4 durch die Monodeuterierung beobachtet. Dieser Trend wurde in Computersimulationen des intramolekularen Energieflusses im Schwingungsquantenzahl-Zustandsraum exakt wiedergegeben. Auf dieser Grundlage wurden die relativen Einflüsse von molekularer Symmetrie und Struktur des Schwingungszustandsraums auf den IVR-Prozeß bewertet. Anhand der Systeme Toluol und $\alpha, \alpha, \alpha$-Trifluortoluol konnte gezeigt werden, daß die Beschleunigung der IVR-Dynamik durch einen internen Rotator nach Anregung aromatischer $\mathrm{CH}$-Streckschwingungen weniger ausgeprägt ist als nach Anregung niederfrequenter Ringmoden. Ein Vergleich der relativen Substituenteneffekte sowie Low Order Coupling-Modellierungen lieferten deutliche Hinweise, daß eine starke Entkopplung der Substituentenfreiheitsgrade von der Schwingungsdynamik des Phenylrestes vorliegt, wenn hochfrequente Phenyl-CH-Schwingungen angeregt werden.

Die Experimente in überkritischer Phase deckten eine Beschleunigung der langsamen IVR-Komponente mit zunehmender Lösungsmitteldichte auf. In Monte-Carlo-Rechnungen wurde eine direkte Korrelation zwischen der IVRGeschwindigkeit und der Frequenz von Solvat-Solvens-Stößen gefunden. Durch Variation der molekularen Umgebung wurde gezeigt, daß die mittlere pro Stoß übertragene Energie keinen meßbaren Einfluß auf die IVR-Dynamik besitzt. Darüber hinaus zeigte sich für Benzolderivate mit polyatomarem Substituenten, daß in kondensierter Phase die durch das Lösungsmittel verursachte Kontraktion der langsamen IVR-Zeitskala die Dynamik verschiedener Moleküle sehr ähnlich werden ließ. In den Gasphasenexperimenten etablierte Substituenteneffekte wurden vom Lösungsmittel „maskiert". 


\section{Kapitel 1}

\section{Einleitung}

Die Lokalisierung von Schwingungsenergie spielt eine zentrale Rolle für den Ablauf chemischer Reaktionen. Mit der Erfindung intensiver Laserlichtquellen schien plötzlich eine modenspezifische Photochemie mittels selektiver Schwingungsanregung in greifbare Nähe gerückt. Es zeigte sich jedoch sehr schnell, daß Energietransferprozesse den Erfolg dieses Konzepts limitieren. Die intramolekulare Umverteilung der Anregungsenergie (IVR, intramolecular vibrational energy redistribution) vermindert die Modenselektivität der Photoreaktion, während die intermolekulare Dissipation der Anregungsenergie (VET, vibrational energy redistribution) die Quantenausbeute limitiert. Ein detailliertes Verständnis dieser beiden Energietransferphänomene ist somit unerläßlich, um sie vorherzusagen und dadurch letztendlich eine Kontrolle über den Reaktionsverlauf zu erhalten.

Die ultimative Herausforderung für die physikalische Chemie besteht darin, Geschwindigkeiten, Ausbeuten, Kanäle und Selektivitäten chemischer Reaktionen in Lösung vorherzusagen. In diesem komplexesten aller denkbaren Reaktionsmedien treten IVR- und VET-Prozess in Konkurrenz zueinander auf, wodurch mechanistische Interpretationen des Energietransfers erheblich erschwert werden. Dennoch konnten erste Erkenntnisse über die Zeitskalen von IVR und VET kleiner aliphatischer und aromatischer Moleküle in kondensierter Phase gewonnen werden.

Einen einfacheren Zugang zu den Einzelprozessen des Energietransfers bieten seit jeher Untersuchungen in der Gasphase, da aufgrund der verminderten Stoßfrequenz die Zeitskala des VET stark verlangsamt ist und von der des IVR separiert werden kann. So konnten für eine Vielzahl von Modellsystemen bereits IVR-Mechanismen formuliert werden [1,2]. Eine Korrelation der IVR-Zeitskalen in der Gas- und der kondensierten Phase gestaltete sich bislang allerdings extrem 
schwierig, da die Daten mit unterschiedlichen experimentellen Methoden erhalten wurden. Daher steht bis heute nicht eindeutig fest, wie und in welchem Maße eine Lösungsmittelumgebung die intramolekulare Energieumverteilung beeinflußt.

Im Rahmen dieser Arbeit konnte erstmals demonstriert werden, daß die IR-Pump-UV-Probe-Spektroskopie hervorragend dafür geeignet ist, die IVRDynamik sowohl isolierter als auch solvatisierter aromatischer Moleküle mit derselben experimentellen Technik zeitaufgelöst und nahezu modenselektiv zu studieren. Experimente in überkritischen Lösungsmitteln bieten sogar die Möglichkeit, den Übergang von gasphasenähnlichen zu flüssigkeitsähnlichen Umgebungsdichten $\mathrm{zu}$ erforschen und somit die Zeitskalen eindeutig zu korrelieren, da etwaige Mechanismenwechsel nicht unbemerkt bleiben können. Auf diese Weise lassen sich darüber hinaus auch eventuell konkurrierende Einflüsse von molekularer Umgebung und chemischer Struktur auf die IVR-Dynamik aufdecken. Die der IRPump-UV-Probe-Spektroskopie inhärente effektive Anregung gestattet es hierbei, selbst in der Gasphase Obertonschwingungen als Ausgangszustand zu präparieren.

Ein Schwerpunkt der vorliegenden Arbeit liegt auf der Auswirkung der chemischen Konstitution eines angeregten Moleküls auf den intramolekularen Energieflußs. Seit in den 70er Jahren die ersten quantitativen IVR-Studien [3] präsentiert wurden, ist die Menge an empirischen Daten zu diesem Thema stetig gewachsen. Mit fortschreitender Entwicklung der experimentellen Techniken konnten immer komplexere Modellsysteme erforscht werden, darunter mizelläre Systeme [4], Farbstoffmoleküle [5] oder über aliphatische Ketten verbundene aromatische Chromophore [6]. Dennoch fehlt noch immer ein globaler Erklärungsansatz für den Zusammenhang zwischen chemischer Struktur und Energierelaxation. So wurde beispielsweise der Einfluß molekularer Symmetrie auf die IVR-Dynamik bislang nur qualitativ analysiert. Diese Arbeit stellt u. a. den ersten direkten Vergleich absoluter IVR-Zeitskalen zweier sich nur in ihrer Symmetrie unterscheidender Modellsysteme vor, anhand dessen in eleganter Weise bestehende Annahmen zu Symmetrierestriktionen des intramolekularen Energieflusses überprüft werden können.

Die Möglichkeiten der Substituentenvariation zur Erforschung von Struktureffekten auf den Energietransfer sind unbestreitbar mannigfaltig. Einen kontrovers diskutierten Aspekt stellt die Beschleunigung der intramolekularen Energieumverteilung in Anwesenheit innerer Rotationsfreiheitsgrade eines Moleküls 
Kapitel 1 Einleitung

dar, welcher durch vergleichende Untersuchungen an zwei chemisch ähnlichen Rotatorsystemen im Rahmen dieser Arbeit näher beleuchtet wird. Einen weiteren interessanten Ansatzpunkt bietet die Schweratomsubstitution an aromatischen Molekülen. Auch hierzu werden im folgenden neue Ergebnisse aus Gasphasenexperimenten vorgestellt.

Neben den Substitutenten der aromatischen Modellsysteme wurde auch die molekulare Umgebung der Aromaten variiert, um den Einfluß nicht-angeregter Solvensmoleküle auf die Schwingungsdynamik angeregter Solvatteilchen zu untersuchen. Dabei stand die Frage, inwiefern die chemische Natur der Umgebung die Energieumverteilung in Benzol verändert, im Vordergrund.

Exakte theoretische Berechnungen der Schwingungsenergieumverteilung sind bis dato nur in aufwendigen quantendynamischen oder molekulardynamischen Simulationen möglich. Systeme mit mehr als zehn Atomen erweisen sich bislang noch als zu komplex, um sie in einer volldimensionalen $a b$ initio-Potentialfäche zu simulieren. Es besteht somit dringender Bedarf an vereinfachenden Ansätzen zur Beschreibung des Energietransfers. Die Beschreibung des intramolekularen Energieflusses als Besetzungsdiffusion im Schwingungszustandsraum stellt ein solches Modell dar [7-9]. Für kleine aliphatische Moleküle konnten hiermit experimentelle Pump-Probe-Signale bereits zufriedenstellend angepaßst werden. Diese Arbeit beschäftigt sich u. a. mit der Weiterentwicklung und Anwendung dieses Modells auf aromatische Moleküle, wobei die hier vorliegenden experimentellen Daten erstmals eine Validierung der Parameter für Systeme dieser Größe ermöglichten.

Die Gliederung der Arbeit gestaltet sich wie folgt. In Kapitel 2 werden die theoretischen Grundlagen des Schwingungsenergietransfers für isolierte und solvatisierte Moleküle dargelegt. Ferner wird das Konzept des Schwingungszustandsraums erläutert, auf welchem die Simulationsrechnungen basieren. Nach der Übersicht über die experimentelle Technik in Kapitel 3 werden in Kapitel 4 die aromatischen Modellsysteme nebst ihrer spektroskopischen Charakteristika vorgestellt. Die Ergebnisse der Pump-Probe-Experimente und Simulationen sind in Kapitel 5 zusammengefaßt. In Kapitel 6 erfolgt die Auswertung der experimentellen Daten. Die hieraus resultierenden Zeitkonstanten bilden die Grundlage der abschließenden Diskussion des intra- und intermolekularen Energietransfers in Kapitel 7. 



\section{Kapitel 2}

\section{Grundlagen}

Der Ausgangspunkt der folgenden Betrachtungen zum Schwingungsenergietransfer sei ein lokal schwingungsangeregtes Molekül. Im Allgemeinen handelt es sich bei solch einem Zustand mit lokalisierter Schwingungsenergie um keinen Eigenzustand des Moleküls; vielmehr stehen dem System prinzipiell zwei Möglichkeiten offen, sich zeitlich zu entwickeln. Zum einen kann die Anregungsenergie innerhalb des Moleküls über alle Schwingungsfreiheitsgrade delokalisiert werden. Dieser Prozess der intramolekularen Schwingungsenergieumverteilung wird im folgenden als IVR (intramolecular vibrational energy redistribution) abgekürzt. Zum anderen ist ein Transfer der überschüssigen Energie aus dem angeregten Molekül in seine Umgebung möglich, ein Phänomen, das im folgenden als VET (intermolecular vibrational energy transfer) bezeichnet wird. Je dichter die molekulare Umgebung, desto ausgeprägter treten diese beiden Prozesse in Konkurrenz zueinander.

Dieses Kapitel widmet sich den theoretischen Grundlagen der beiden Energieumverteilungsprozesse. Hierbei liegt das Hauptaugenmerk auf der intramolekularen Dynamik, da sie das zentrale Thema der vorliegenden Arbeit darstellt. Quantenmechanische Formulierungen und mechanistische Konzepte des IVR werden zunächst für den idealisierten Fall des isolierten Moleküls erörtert. Unter den vorgestellten Modellen ist die Beschreibung des intramolekularen Energieflusses im Schwingungszustandsraum besonders hervorzuheben; sie bildet die Grundlage von IVR-Simulationsrechnungen auf empirischer Basis, deren Durchführung ebenfalls in diesem Kapitel beschrieben wird. Anschließend wird kurz auf etablierte Modelle des intermolekulareren Energietransfers eingegangen. In diesem Zusammenhang werden auch mögliche Beeinflussungen des IVR-Prozesses durch die molekulare Umgebung benannt. 


\subsection{Intramolekulare Dynamik}

\subsubsection{IVR im isolierten Molekül}

Der IVR-Prozess wird meistens als irreversible intramolekulare Energieumverteilung eines hellen Zustands auf eine Anzahl isoenergetischer dunkler Zustände desselben Moleküls formuliert. Dabei sind die Begriffe heller und dunkler Zustand in enger Anlehnung an experimentelle IVR-Studien geprägt. Als hell werden Zustände bezeichnet, die direkt durch optische Anregung populiert werden können. In den meisten Fällen ist der Anteil heller Zustände an der Gesamtzustandsdichte eines Moleküls gering, da es sich um Zustände mit großem Übergangsmatrixelement zum Grundzustand handeln muß. Der überwiegende Teil der Zustandsdichte setzt sich aus dunklen Zuständen zusammen, die aufgrund optischer Auswahlregeln und/oder kleinen Überlappintegrals mit dem Grundzustand nicht durch optisch induzierte Übergänge besetzt werden können.

Im mikroskopischen Bild läßst sich der IVR-Prozess als Umverteilung von in einer Bindung lokalisierter Anregungsenergie auf das gesamte molekulare Gerüst veranschaulichen. Die lokale Bewegung der angeregten Bindung breitet sich unweigerlich auf das gesamte Netzwerk gekoppelter Oszillatoren aus. Für ein isoliertes Molekül ist die Umverteilung der Schwingungsenergie abgeschlossen, wenn alle Schwingungsfreiheitsgrade equilibriert und statistisch populiert sind.

\section{Quantenmechanische Formulierung des IVR-Prozesses}

Die quantenmechanische Betrachtung des dynamischen Phänomens IVR fordert, daß der helle Zustand mit lokalisierter Anregungsenergie nicht zu den Eigenzuständen des Systems gehört, da letztere inhärent statisch sind. Jedoch läßst sich ein beliebiger heller Zustand $\Psi$ zum Zeitpunkt der Anregung $(t=0)$ als Superposition einer Menge $i$ solcher Eigenzustände gemäß

$$
\Psi(t=0)=\sum_{i} c_{i} \psi_{i}
$$

formulieren, wobei für die Koeffizienten $c_{i}$, die den Überlapp des jeweiligen Eigenzustands mit dem hellen Zustand beschreiben,

$$
c_{i}=\left\langle\psi_{i} \mid \Psi(0)\right\rangle
$$


gilt. Die zeitabhängige Entwicklung des Superpositionszustandes ist durch

$$
\Psi(t)=\sum_{i} c_{i} \psi_{i} \exp \left(\frac{-\mathrm{i} E_{i} t}{\hbar}\right)
$$

gegeben. $E_{i}$ bezeichnet die Energieeigenwerte der am hellen Zustand beteiligten Eigenfunktionen $\psi_{i}$. Die intramolekulare Dynamik ist dann in der zeitabhängigen Wahrscheinlichkeitsverteilung

$$
\begin{aligned}
P(t) & =\Psi^{*}(t) \Psi(t) \\
& =\sum_{i}\left|c_{i}\right|^{2}\left|\psi_{i}\right|^{2}+\sum_{i>j}\left[c_{i} c_{j}^{*} \psi_{i} \psi_{j}^{*} \exp \left(\frac{-\mathrm{i}\left(E_{j}-E_{i}\right) t}{\hbar}\right)+\text { c. c. }\right]
\end{aligned}
$$

und der zeitabhängigen Autokorrelation

$$
\langle\Psi(t) \mid \Psi(0)\rangle=\sum_{i}\left|c_{i}\right|^{2} \exp \left(\frac{-\mathrm{i} E_{i} t}{\hbar}\right)
$$

impliziert. Für große Moleküle strebt die Überlebenswahrscheinlichkeit des hellen Zustands $\Psi(0)$ zu unendlich langen Zeiten gegen Null.

\section{Helle Zustände nullter Ordnung}

In zeitaufgelösten Untersuchungen zum IVR wird der Anfangszustand $\Psi(0)$ mittels eines ultrakurzen Laserpulses präpariert. Solche Pulse weisen aufgrund der Unschärferelation eine Verbreiterung ihres Spektrums auf. I. A. schließt die spektrale Breite eine Vielzahl nahezu isoenergetischer heller Eigenzustände ein. Dabei ist jeder dieser hellen Eigenzustände als Linearkombination in der gewählten Schwingungsbasis, z. B. der Basis der Normalmoden, darstellbar. Bei ausreichend geringer Pulsdauer erfolgt im Experiment eine kohärente Anregung aller hellen Zustände unter der Einhüllenden des Laserspektrums. Der so präparierte nichtstationäre Schwingungszustand kann als Wellenpaket entsprechend Gl. (2.1) aufgefaßt werden.

Von den kohärent angeregten Eigenzuständen tragen nur diejenigen außer zur Phase auch zur Amplitude des Wellenpakets bei, deren Dipolmoment mit dem einfallenden elektromagnetischen Feld wechselwirken kann. Aus diesem Grund ähnelt der nichtstationäre Schwingungszustand zum Zeitpunkt $t=0$ einem einzigen hellen Zustand, der in der gewählten Basis beschrieben werden kann, z. B. 
dem ersten Oberton einer CH-Streckschwingung. Dieser Zustand, dem das kohäherent erzeugte Wellenpaket bei $t=0$ ähnelt, wird als heller Zustand nullter Ordnung (zeroth order bright state, ZOBS) bezeichnet ${ }^{1}$.

Es sei an dieser Stelle angemerkt, daß viele IVR-Studien in der Frequenzdomäne, bei denen hochaufgelöste Spektren interpretiert werden, mit kontinuierlichen, monochromatischen Laserlichtquellen durchgeführt werden. Mit solchen Lasern werden jedoch typischerweise Eigenzustände des Moleküls angeregt. Das bedeutet, daß der oben beschriebene Zustand $\Psi(0)$ mit lokalisierter Anregung, der mit der zeitabhängigen Relaxation assoziiert ist, in diesen Experimenten zu keinem Zeitpunkt realisiert wird. Vielmehr werden die Natur des hellen Zustands und die damit verbundene intramolekulare Dynamik indirekt aus den Linienaufspaltungen und relativen Linienintensitäten der Spektren erhalten. Hierbei geben die Aufspaltungen Auskunft über die $E_{i}$, während die Intensitäten mit den $\left|c_{i}\right|^{2}$ verknüpft sind.

\section{Realisierung des ZOBS}

Die Natur des hellen Zustands im Experiment und damit auch seine intramolekulare Dynamik werden signifikant von der Art seiner Präparation bestimmt. Im großen und ganzen lassen sich helle Zustände mit ultrakurzen Laserpulsen auf drei Arten realisieren:

- Durch direkte Einphotonenanregung sind experimentell diejenigen Schwingungszustände am besten zugänglich, die eine hohe Frequenz und stark anharmonischen Charakter bei kleiner Änderung der Gesamtquantenzahl im Vergleich zum Grundzustand besitzen. Hierfür kommen vor allem hydridische Streckschwingungen und deren Obertöne in Frage, weswegen experimentelle Studien sich bis dato besonders auf die IVR-Dynamik von RHModen konzentrieren. Auch die IR-Pump-UV-Probe-Spektroskopie, die in dieser Arbeit eingesetzt wurde, arbeitet mit hellen Zuständen dieser Kategorie.

\footnotetext{
${ }^{1}$ Strenggenommen wird der ZOBS somit im Experiment nicht angeregt. Aufgrund der großen Ähnlichkeit der Schwingungsbewegung zum Zeitpunkt $t=0$ wird der Begriff ZOBS im folgenden trotzdem synonym für den experimentell präparierten nichtstationären Schwingungszustand verwendet.
} 
- In Systemen mit bindenden angeregten elektronischen Zuständen kann die Methode des Franck-Condon-Pumpens, kombiniert mit hochaufgelöster Fluoreszenz oder stimulierter Emission, angewandt werden, um helle $\mathrm{Zu}$ stände zu präparieren, die durch direkte Einphotonenabsorption nicht zugänglich sind. Hierbei werden im Zuge der elektronischen Anregung FranckCondon-aktive Moden populiert, die auch nach der Rückkehr des Systems in den Grundzustand signifikant zum hellen Zustand beitragen.

- Die Methode der Zweiphotononenanregung gewährt die größte Flexibilität bei der Wahl des hellen Zustands, ist aber experimentell schwieriger umzusetzen. Hiermit sind u. a. helle Zustände zugänglich, deren Bevölkerung durch Einphotonenabsorption optisch verboten ist. Auch können isoenergetische Kombinationstöne durch geeignete Wahl der Pumpwellenlängen voneinander getrennt präpariert werden.

\section{Symmetrierestriktionen}

Quantenmechanisch betrachtet findet ein Übergang zwischen zwei durch die Wellenfunktionen $\psi_{1}$ und $\psi_{2}$ definierten Zuständen eines Moleküls dann statt, wenn das Integral

$$
\int \psi_{1}^{*} \hat{O} \psi_{2} \mathrm{~d} \mathbf{r}
$$

einen von Null verschiedenen Wert annimmt. $\hat{O}$ steht hierbei für einen beliebigen Operator des Übergangsmoments. Eine analytische Evaluation des Integrals und damit einhergehende rechnerische Bestimmung der Auswahlregeln für Zustandsänderungen ist nur für einfache Systeme bei bekannter funktionaler Form der $\psi_{i}$ möglich. In komplexeren Fällen helfen Symmetriebetrachtungen bei der Herleitung der Auswahlregeln, so daß eine mathematische Lösung von Gl. 2.6 umgangen werden kann. Generell haben Integrale dieses Typs nur dann von Null verschiedene Lösungen, wenn der Integrand totalsymmetrisch ist, d. h. wenn das Produkt der irreduziblen Darstellungen $\Gamma$ von $\psi_{1}, \psi_{2}$ und $\hat{O}$ die totalsymmetrische Darstellung $\Gamma^{(s)}$ der entsprechenden Punktgruppe enthält.

$$
\begin{array}{rlrl} 
& \Gamma_{1} \otimes \Gamma_{2} \otimes \Gamma_{\hat{O}} & \supseteq \Gamma^{(s)} \\
\text { bzw. } & \Gamma_{1} \otimes \Gamma_{2} \supseteq \Gamma_{\hat{O}}
\end{array}
$$


Anharmonische Kopplungen, welche die intramolekulare Energieumverteilung ermöglichen, bedürfen keiner Initiierung durch ein äußeres elektromagnetisches Feld. Für diese strahlungslosen Übergänge entfällt daher im Übergangsintegral (Gl. 2.6) der Operator $\hat{O}$. Es bleibt lediglich das Überlappungsintegral

$$
\int \psi_{1}^{*} \psi_{2} \mathrm{~d} \mathbf{r}
$$

der Wellenfunktionen zu evaluieren. Ein totalsymmetrischer Integrand erfordert in diesem Fall, daß die Symmetrien der Zustände $\psi_{1}$ und $\psi_{2}$ gleich sind. Somit laufen anharmonische Kopplungen in isolierten Molekülen theoretisch unter Symmetrieerhalt ab, wodurch die Anzahl koppelnder dunkler Zustände je nach Symmetrie des Systems und des hellen Zustands eingeschränkt sein kann.

\section{Fermis Goldene Regel}

Die Existenz des Phänomens der intramolekularen Schwingungsenergieumverteilung wurde noch Mitte des 20. Jahrhunderts kontrovers diskutiert. 1968 zeigten Bixon und Jortner anhand der bis dahin gesammelten experimentellen Daten, daß diese Art von strahlungslosen Schwingungsübergängen existieren muß. Sie stellten das erste Modell zur Beschreibung des IVR-Prozesses als über Kopplungen zwischen Zuständen vermittelten Energiefluß vor [10]. Über einen störungstheoretischen Ansatz 1. Ordnung konnten sie eine Fermis Goldener Regel (FGR) entsprechende Geschwindigkeitskonstante

$$
k_{\mathrm{FGR}}=\frac{2 \pi}{\hbar} \rho\langle V\rangle^{2}
$$

formulieren, welche den Energiefluß aus einem hellen Zustand $\Psi(t=0)$ in eine Mannigfaltigkeit von dunklen Hintergrundzuständen der Gesamtzustandsdichte $\rho$, die mit einer mittleren Kopplungsstärke $\langle V\rangle$ an den hellen Zustand koppeln, beschreibt. In diesem Fall nimmt das Betragsquadrat der Autokorrelation (s. Gl. 2.5) monoexponentiell ab:

$$
|\langle\Psi(t) \mid \Psi(0)\rangle|^{2} \propto \exp \left(-k_{\mathrm{FGR}} t\right)
$$

Diese Formulierung der Geschwindigkeitskonstanten ist als oberes statistisches Limit des IVR-Prozesses einzustufen. Es konnte inzwischen gezeigt werden, daßs in den meisten Fällen nicht die Gesamtzustandsdichte, sondern nur ein Teil davon 


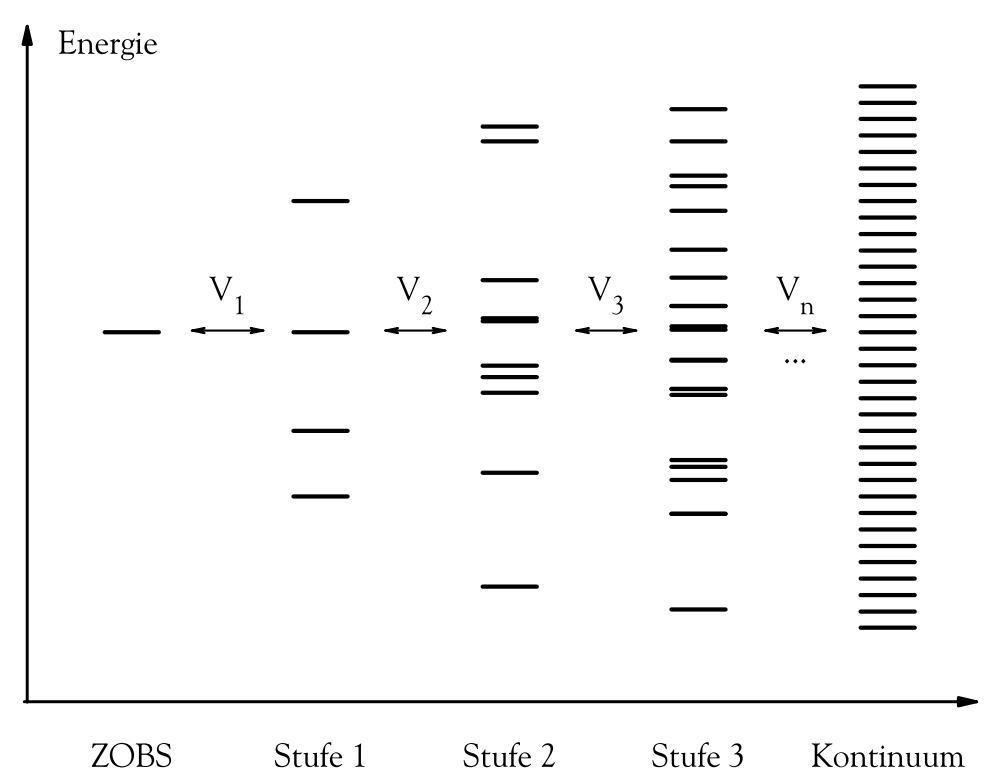

Abbildung 2.1: Graphische Darstellung des IVR-Stufenmodells

für die IVR-Dynamik verantwortlich ist. Zudem weist eine Vielzahl von untersuchten Systemen nicht-monoexponentielles IVR-Verhalten auf, woraus geschlußfolgert werden darf, daß die Relaxation in mehreren Schritten abläuft. Die folgenden Abschnitte 2.1.2 und 2.1.3 stellen Beschreibungsmodelle entsprechender hierarchischer Kopplungsstrukturen vor.

\subsubsection{Das IVR-Stufenmodell}

Im Gegensatz zur FGR-Beschreibung, in der die Schwingungsenergie in einem Schritt auf das gesamte Bad von dunklen Hintergrundzuständen umverteilt wird, wird in hierarchischen IVR-Modellen die Gesamtzustandsdichte sukzessive erschlossen. Einen Ansatz zur Veranschaulichung schrittweiser IVR-Dynamik stellt das ,Stufen-Modell“ (engl. tier model) dar [11], welches inzwischen allgemein akzeptiert ist. Abb. 2.1 hilft, das Konzept zu verdeutlichen.

Die Badzustände werden anhand der Stärke ihrer Kopplung an den ZOBS gruppiert. Aus dem zum Zeitpunkt $t=0$ präparierten hellen Zustand mit lokalisierter 
Kapitel 2 Grundlagen

Anregung fließt die Energie bevorzugt in einige wenige, nahezu isoenergetische Badzustände, weil diese starke anharmonische Kopplungen $\mathrm{V}_{1}$ mit dem ZOBS aufweisen. Diese Untergruppe der Gesamtzustandsdichte stellt den ersten tier dar. In der Literatur werden die Zustände der ersten Umverteilungsstufe z.T. auch als Durchgangszustände (,doorway states“) bezeichnet, da sie unweigerlich passiert werden müssen, bevor die Umverteilung fortschreiten kann. Aus der ersten Stufe fließt die Energie wiederum über anharmonische Kopplungen (großes $\mathrm{V}_{2}$ ) in den zweiten, größeren Satz quasi-isoenergetischer Zustände, von dort in den dritten usw. Auf diese Weise wird sukzessive die Zustandsdichte innerhalb des Energieintervalls bevölkert, bis das Kontinuum aller Zustände erreicht ist. Der sequentielle Energiefluß ist nur gewährleistet, wenn für die Kopplung des ZOBS an die einzelnen tier gilt, daß

$$
\text { ZOBS } \leftrightarrow \text { tier } 1>\text { ZOBS } \leftrightarrow \text { tier } 2>\text { ZOBS } \leftrightarrow \text { tier } 3>\text { etc. }
$$

ist. IVR innerhalb der Zustände einer Umverteilungsstufe wird in diesem Bild einer Relaxationskaskade nicht berücksichtigt.

\subsubsection{Der Quantenzahl-Zustandsraum}

Eine alternative, mit dem IVR-Stufenmodell verwandte Beschreibung schrittweiser IVR-Dynamik bezieht sich auf den Quantenzahl-Zustandsraum (engl.: quantum number space, QNS) [12].

Der QNS ist der von den $s=3 N-6$ Schwingungsfreiheitsgraden aufgespannte Zustandsraum eines Moleküls mit $N$ Atomen. Die Basis der $s$ Freiheitsgrade kann hierbei aus Normalmoden, Lokalmoden oder diagonal anharmonischen Normalmoden bestehen. In dieser Arbeit wurde die harmonische Normalmodenrepräsentation des QNS verwendet.

In Abb. 2.2 ist beispielhaft ein dreidimensionaler QNS $(s=3)$ skizziert. Die im folgenden vorgestellten charakteristischen Eigenschaften des 3D-Modells sind auf beliebig dimensionale Systeme übertragbar.

Die Achsen des QNS skalieren mit der Anzahl $v$ von Schwingungsquanten in einer Normalmode $\nu_{i}(i=1 \ldots s)$. Jeder Schwingungszustand $\left|v\left(\nu_{1}\right) v\left(\nu_{2}\right) v\left(\nu_{3}\right)\right\rangle$ entspricht somit einem kartesischen Gitterpunkt. Den Ursprung des QNS bildet der Grundzustand $|000\rangle$. Isoenergetische Zustände liegen auf einer Energieschale um den Ursprung. In harmonischer Näherung ist diese Schale planar; würden Anharmonizitäten berücksichtigt, erschiene die Schale konkav verformt. 
Die Besonderheit des QNS sind seine Begrenzungen, die es erlauben, drei verschiedene Arten von Gitterpunkten zu unterscheiden.

- Innere Zustände (interior states): Die Schwingungsquantenzahlen $v$ aller Moden sind bei diesen Zuständen von Null verschieden. In polyatomaren Systemen konstituieren bei chemisch signifikanten Energien diese Zustände den Hauptanteil der Zustandsdichte.

- Kantenzustände (edge states): In diesen Zuständen ist nur eine der $s$ Schwingungsquantenzahlen von Null verschieden, so daß die zugehörigen Gitterpunkte auf den Achsen des QNS liegen. Kantenzustände entsprechen reinen Obertonschwingungen, wie sie experimentell leicht zugänglich sind (s. S. 10).

- Oberflächenzustände (surface states): Hier ist mehr als eine Schwingungsquantenzahl von Null verschieden. Zu den Oberflächenzuständen zählen somit Kombinationstöne, an denen maximal $s-1$ Moden beteiligt sind.

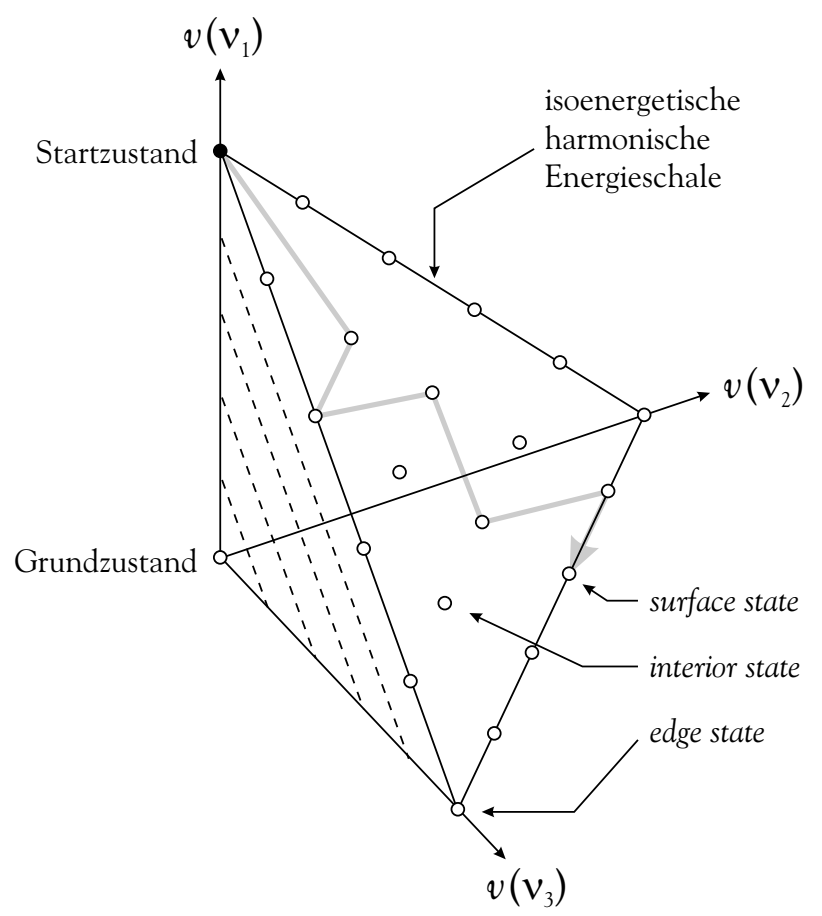

Abbildung 2.2: Schematische Darstellung eines dreidimensionalen QNS 
Die Stärke der Kopplung $V$ zwischen zwei Zuständen kann aus der QNSDarstellung sehr anschaulich abgeleitet werden. Aus störungstheoretischer Sicht skaliert der Überlapp zwischen zwei Zuständen $i$ und $j$ negativ exponentiell mit der Ordnung $\Delta n$ eines Übergangs. Diese ist als die Summe aller Quantenzahlendifferenzen zwischen $i$ und $j$,

$$
\Delta n=\sum_{s}\left|v\left(\nu_{s}\right)_{i}-v\left(\nu_{s}\right)_{j}\right|
$$

definiert. Im QNS entspricht die Ordnung der Kopplung gerade dem geometrischen Abstand der Gitterpunkte $i$ und $j$. Darüber hinaus gilt das Energielückengesetz (engl. energy gap law), daßs eine exponentielle Abnahme der Kopplungsstärke mit zunehmender Energiedifferenz $E_{i}-E_{j}$ festlegt. Dies ist im Bild des QNS als Energietransfer zwischen unterschiedlichen Energieschalen zu verstehen. Damit liegt hier ebenfalls eine Abhängigkeit der Kopplungsstärke vom Abstand der Gitterpunkte, diesmal als Lot zwischen entsprechenden Energieschalen gemessen, vor. Zusammenfassend koppeln also Zustände im QNS umso besser, je kürzer ihr Verbindungsvektor bzw. ihr Abstand im QNS und Energieraum ist.

Als Konsequenz kann der IVR-Prozess als Diffusion der Besetzung eines Startgitterpunktes im QNS aufgefaßt werden. Demzufolge wäre für einen Kombinationston (inneren Zustand) eine schnellere IVR-Dynamik als für einen reinen Oberton (Kantenzustand) zu erwarten, da letzterem nur die Zustände des ersten $2^{s}$-tanden - in Abb. 2.2 des ersten Oktanden - als Kopplungspartner zur Verfügung stehen, während ersterer in alle Richtungen diffundieren kann. In Abb. 2.2 ist beispielhaft die Relaxation des $5 \nu_{1}$-Obertons als zufällige Bewegung (,random walk") durch den QNS skizziert (grauer Pfeil).

Bei genauerer Betrachtung zeigt sich, daß eine 2D-Projektion des QNS zurück zur Stufenrepräsentation des vorigen Abschnitts führt. Eine Projektionsachse stellt die Energie (Normalenvektor der ZOBS-Energieschale) dar, die andere den QNS-Abstand $\Delta n$ ohne Richtungsinformation. Eine Anisotropie der Gitterpunktverteilung im QNS ist hierbei nur indirekt berücksichtigt, indem die Matrixelemente $V_{n}$ variieren können.

\subsubsection{Das empirische QNS-Modell}

Die Beschreibung des intramolekularen Schwingungsenergietransfers als Umverteilung von Zustandsbesetzungen im QNS ermöglicht eine quantitative Modellie- 
rung von IVR-Prozessen. Ein hierauf beruhendes empirisches Modell zur Simulation von IVR im Schwingungszustandsraum wurde in Ref. [13] formuliert und bereits für kleine Alkyliodide (1-3 C-Atome) getestet.

Die Geschwindigkeit des Übergangs von einem Zustand $i$ zu einem Zustand $j$ ist abhängig von der Stärke der Kopplung zwischen diesen Zuständen, ausgedrückt durch die Kopplungskonstante $k_{i j}$. Sind alle Kopplungskonstanten bekannt, kann die zeitliche Änderung der Besetzungswahrscheinlichkeit $p$ des Zustands $i$ als Summe über alle bevölkernden und entvölkernden Prozesse ausgedrückt werden

$$
\frac{\mathrm{d} p_{i}}{\mathrm{~d} t}=\sum_{j}\left[k_{i j} p(j, t)-k_{j i} p(i, t)\right] .
$$

Die Lösung dieser IVR-Mastergleichung ist möglich, wenn die zustandsspezifischen Kopplungskonstanten $k_{i j}$ bestimmt werden können. Im folgenden wird eine empirische Formel für $k_{i j}$ hergeleitet und begründet, auf deren Grundlage IVRProzesse mit relativ geringem Rechenzeitaufwand simuliert werden können.

Ausgangspunkt ist die Wechselwirkung eines hellen Zustands $i$ mit einer Mannigfaltigkeit von Hintergrundzuständen $j$. Nach Bixon und Jortner [10] gilt ein Fermis Goldener Regel analoger Ausdruck für die Entvölkerung des Zustands $i$

$$
k_{\mathrm{FGR}}=\frac{2 \pi}{\hbar} \rho\langle V\rangle^{2}
$$

Gleichung (2.14) enthält neben der Zustandsdichte $\rho$ der Hintergrundzustände auch eine mittlere Kopplungskonstante $\langle V\rangle$, die zusammenfassend die Kopplung des hellen Zustands an die $N$ einzelnen dunklen Zustände beschreibt

$$
\langle V\rangle^{2}=\sum_{j=1}^{N} \frac{V_{i j}^{2}}{N}
$$

Für die Berechnung zustandsspezifischer Kopplungskonstanten ist die Formulierung einer mittleren Kopplungskonstante allerdings zu vereinfachend, daher ist Gleichung (2.15) in Gleichung (2.14) einzusetzen

$$
k_{\mathrm{FGR}}=\sum_{j=1}^{N} k_{i j}=\frac{2 \pi}{\hbar} \sum_{j=1}^{N}\left(\frac{\left|V_{i j}\right|^{2}}{N}\right) \cdot \rho .
$$


Der Vergleich der Summationen in Gleichung (2.16) ergibt die Bestimmungsgleichung der zustandsabhängigen Entvölkerungskonstanten für den Fall isoenergetischer Hintergrundzustände

$$
k_{i j}=\frac{2 \pi}{\hbar} \cdot \frac{\rho}{N} \cdot\left|V_{i j}\right|^{2} .
$$

Die Arbeiten von Parmenter et al. [14] legen eine exponentielle Skalierung der Matrixelemente $V_{i j}$ mit dem Abstand der Schwingungszustände im QNS nahe

$$
V_{i j}=V_{k} \mathrm{e}^{-\frac{1}{2} \gamma[\Delta n-3]} .
$$

$\Delta n$ entspricht als Summe der absoluten Differenzen aller Quantenzahlen der $\mathrm{Zu}$ stände $i$ und $j$ der Ordnung der Kopplung, ist also dem Parameter $Q$ bei Wolynes gleichzusetzen. Der Abklingparameter $\gamma$ gewichtet diese Entfernung der Zustände im QNS bezüglich der Kopplungswahrscheinlichkeit. Im Fall einer kubischen Kopplung $(\Delta n=3)$ gilt $V_{i j}=V_{k}$, daher entspricht $V_{k}$ mit Werten zwischen 3 und $10 \mathrm{~cm}^{-1}$ einem typischen kubischen Kopplungsterm. Einsetzen von Gleichung (2.18) in Gleichung (2.17) ergibt die Kopplungskonstanten $k_{i j}$ für alle Übergänge, einschließlich der Übergänge zwischen Hintergrundzuständen

$$
k_{i j}=\frac{2 \pi}{\hbar} \cdot \frac{\rho}{N} \cdot\left|V_{k}\right|^{2} \mathrm{e}^{-\gamma[\Delta n-3]} .
$$

Der Wert der Zustandsdichte $\rho$ entspricht im Fall isoenergetischer Kopplung dem Wert der Gesamtzahl der Zustände $N$, so daß der zweite Term in Gleichung (2.19) lediglich die Dimension einer reziproken Energie beiträgt.

Wird nun nicht eine isoenergetische "Schale" im QNS, sondern ein Energieintervall der Breite $\Delta E$ betrachtet, muß auch der Abstand der Zustände $\Delta E_{i j}=\left|E_{j}-E_{i}\right|$ berücksichtigt werden (nichtisoenergetischer Fall). Hier gilt das energy gap law (vgl. Abschn. 2.1.3). Es liegt eine exponentielle Abhängigkeit der Kopplung von der Energielücke vor,

$$
k_{i j} \propto \mathrm{e}^{-\lambda\left|\Delta E_{i j}\right|}
$$

wobei der Ausdruck mit $\lambda$ gewichtet ist. Dadurch wird die Zahl $N_{\text {ges }}$ der zugänglichen Zustände begrenzt. Sie ergibt sich näherungsweise aus

$$
N_{\text {ges }}=\int_{E_{i}-\delta}^{E_{i}+\delta} \rho(E) \mathrm{d} E \quad \text { mit } \quad \delta \approx 2 / \lambda .
$$


Unter Annahme einer im betrachteten Energieintervall unabhängigen Zustandsdichte folgt daraus

$$
N_{\text {ges }} \approx \rho \frac{4}{\lambda}
$$

Zusammenfassung der Gleichungen (2.19)-(2.22) liefert einen Ausdruck für alle Kopplungskonstanten $k_{i j}$, der sowohl die Abhängigkeit vom Abstand der koppelnden Zustände im QNS als auch im Energieraum erfaßt

$$
k_{i j}=\frac{2 \pi}{\hbar} \cdot \frac{\lambda}{4} \cdot\left|V_{k}\right|^{2} \cdot \mathrm{e}^{-\gamma[\Delta n-3]} \mathrm{e}^{-\lambda\left|\Delta E_{i j}\right|} .
$$

Die Gesamtentvölkerungskonstante des Zustands $i$ ergibt sich durch Summation über alle entvölkernden Kopplungskonstanten

$$
k_{i i}=-\sum_{j \neq i} k_{i j}
$$

Des weiteren wird vorausgesetzt, daß die Kopplungswahrscheinlichkeiten für beide Richtungen gleich groß sind,

$$
k_{i j}=k_{j i}
$$

d. h. es liegt mikroskopische Reversibilität, aber kein detailliertes Gleichgewicht vor.

Gehört das betrachtete Molekül zu einer nicht-entarteten Punktgruppe, ist die Inkorporierung der auf S. 11 erwähnten Symmetrierestriktion in das QNSDiffusionsmodell leicht zu realisieren. Über die irreduziblen Darstellungen der einzelnen Normalmoden kann die Symmetrie jedes Zustandes ermittelt werden. Für Kopplungen zwischen Zuständen unterschiedlicher Symmetrie $S$ werden die entsprechenden Kopplungskonstanten Null gesetzt. Handelt es sich allerdings um ein Molekül mit entarteter Punktgruppe, gestaltet sich die Bestimmung der Zustandssymmetrien schwieriger. ${ }^{2}$

In Form der Matrix K steht ein kompletter Satz von Kopplungskonstanten zur Berechnung sämtlicher Übergangswahrscheinlichkeiten im betrachteten Teil des QNS zur Verfügung.

\footnotetext{
${ }^{2}$ Die in dieser Arbeit vorgestellten QNS-Simulationen an Benzol und Benzol-d $\mathrm{d}_{1}$ wurden aus diesem Grund unter Vernachlässigung der Symmetrierestriktionen durchgeführt.
} 


\subsubsection{Simulation von IVR-Prozessen im QNS}

Ist die Kopplungsmatrix $\mathbf{K}$ bekannt, läßt sich eine vollständige Simulation des IVR-Prozesses im Bild der Normalmoden durchführen. Eine derartige Simulationsrechnung besteht aus sechs Schritten:

1. Wahl des Startzustands: Für den ZOBS wird die Besetzung der Normalmoden frei gewählt. Dieser Zustand wird zusammen mit seiner Gesamtenergie, die in der Simulation der Pumpenergie entspricht, abgespeichert.

2. Hintergrundzustände: Anschließend sind die Quantenzahlen und Energien aller $N$ dunklen Schwingungszustände zu ermitteln, die im betrachteten Energieintervall zur Zustandsdichte des Moleküls beitragen. Das Ergebnis ist eine Matrix $\mathbf{Q}$ mit $N$ Zeilen, in denen die Normalmodenbesetzungen und die zugehörigen Energien der Hintergrundzustände abgespeichert werden.

2a. Isotrope Reduktion des QNS (optional): Um IVR in Systemen mit hohen Zustandsdichten $\left(\rho>100\right.$ pro $\left.\mathrm{cm}^{-1}\right)$ in vertretbarer Zeit zu simulieren, muß die Dimension der Kopplungsmatrix K auf maximal $12000 \cdot 12000$ limitiert werden. Für Rechenzeiten von weniger als $24 \mathrm{~h}$ empfiehlt es sich, die Dimension von $\mathbf{K}$ auf $<10000^{2}$ zu begrenzen. Eine Möglichkeit der Reduktion stellt die Verkleinerung des Energieintervalls um die Anregungsenergie dar. Für realistische Simulationen sind allerdings Energieschalen von mindestens $10 \mathrm{~cm}^{-1}$ Breite erstrebenswert, daher ist eine isotrope Verdünnung des QNS vorzuziehen. Zu diesem Zweck werden die unter 2. zusammengestellten Hintergrundzustände nach ihrer Energie geordnet und abhängig von der Zustandsdichte jeder $i$-te $(i=2,3,4 \ldots)$ Zustand eliminiert. Die molekülspezifische Struktur des QNS bleibt hierbei erhalten.

3. Berechnung der Kopplungsmatrix: Es erfolgt die Berechnung der Kopplungsmatrixelemente $k_{i j}$ mittels der IVR-Mastergleichung unter Berücksichtigung der Randbedingungen (Gleichungen (2.24) und (2.25)). In der Diagonale der resultierenden $(N \times N)$-Matrix $\mathbf{K}$ stehen die Gesamtentvölkerungskonstanten $k_{i i}$.

4. Präparation der Anfangssituation im QNS: Zur Beschreibung des Anfangszustands dient ein Vektor $\mathbf{p}_{0}$, der die Anfangsbesetzung jedes Zustands im Energieintervall enthält. Die Numerierung der Zustände sollte hierbei 
mit der Numerierung der Zustände in Q übereinstimmen. Zum Zeitpunkt $t=0$ besitzt nur das dem ZOBS entsprechende Element den Wert 1, alle anderen Elemente haben den Wert 0. Dies entspricht der Besetzung eines einzigen Gitterpunkts im QNS.

5. Propagation der Besetzung im QNS: Die Besetzungsverteilung zum Zeitpunkt $t$ ergibt sich aus der Lösung des Differentialgleichungssystems

$$
\frac{\mathrm{d} \mathbf{p}}{\mathrm{d} t}=\mathbf{K} \cdot \mathbf{p} .
$$

Bei $t \rightarrow \infty$ liegt eine homogene Besetzungsverteilung für alle die Symmetrierestriktionen erfüllenden Gitterpunkte mit den Wahrscheinlichkeiten $p_{i}(t \rightarrow \infty)=N^{-1} \forall i$ vor.

6. Berechnung der Besetzung aller Normalmoden: Projektion der Wahrscheinlichkeitsverteilung der Gitterpunkte auf die jeweilige Normalmode $\nu$

$$
P_{\nu}(t)=\sum_{i=1}^{N} p_{i}(t) Q_{i, \nu}
$$

liefert die Besetzung der einzelnen Schwingungsfreiheitsgrade zu jedem Zeitpunkt $t$. Auf diese Weise ist es möglich, die Besetzungsänderungen einer Franck-Condon-aktiven Mode zu simulieren und mit den transienten Absorptionen des Experiments zu vergleichen.

\subsubsection{Bekannte Strukturfaktoren}

Das ultimative Ziel sämtlicher Studien zum IVR-Prozess, sprich die Vorhersage und Steuerung desselben, ist noch lange nicht erreicht. Allerdings konnten in der Vergangenheit einige Faktoren identifiziert werden, die unwiderlegbar einen Einfluß auf den intramolekularen Energiefluß haben. Sowohl dem Chemiker als auch dem Spektroskopiker drängt sich in diesem Zusammenhang das Konzept funktioneller Gruppen auf. Hinweise auf einige solcher Strukturfaktoren wurden bereits gefunden:

Parmenter et al. konnten den IVR-beschleunigenden Effekt eines internen Methylrotators für elektronisch angeregte Sechsringaromaten am Beispiel des 
p-Fluortoluols belegen [15-18]. Mit einem Modell intramolekularer „Stöße“ zwischen der Methylgruppe und den orthoständigen Wasserstoffatomen des Phenylrings konnten die experimentellen Daten semiquantitativ erklärt werden. Durch diese Wechselwirkungen könnte die große Anzahl von Rotationszuständen zur effektiven Zustandsdichte im FGR-Bild beitragen. Allerdings ist in einer Studie an terminalen Acetylenen keine analoge IVR-Beschleunigung beobachtet worden [19-21].

Ähnlich wie die innere Rotation einer Methylgruppe können auch andere Torsionsmoden mit großer Amplitude, z. B. die Rotation um einzelne CO- oder CC-Bindungen, den IVR-Prozess beeinflussen. Der Effekt solcher allgemein als flexible Zentren bezeichneter Strukturmerkmale konnte für einige kurzkettige Kohlenwasserstoffe experimentell nachgewiesen werden [19, 22, 23]. Dabei spielt die räumliche Nähe des flexiblen Zentrums zur lokal angeregten $\mathrm{CH}-$ Streckschwingung eine nachweisliche Rolle [24].

Als Schweratomeffekt wird die Verlangsamung der intramolekularen Energieumverteilung bei Substitution von Gerüstatomen durch schwerere Elemente der gleichen Gruppe bezeichnet. Dieses Phänomen wurde beispielsweise von Lehmann et al. im Falle der Relaxation von $\mathrm{CH}$-angeregten terminalen Acetylenen des Typs $\left(\mathrm{H}_{3} \mathrm{C}\right)_{3} \mathrm{X}-\mathrm{CCH}(\mathrm{X}=\mathrm{C}, \mathrm{Si}, \mathrm{Sn})$ beobachtet $[25,26]$.

\subsection{Intermolekulare Dynamik}

Intermolekularer Schwingungsenergietransfer (VET) von einer angeregten Spezies in seine Umgebung führt ultimativ zu einer Equilibrierung der Anregungsenergie im gesamten System. Die Natur der chemischen Umgebung - Gasphase, überkritische Phase oder flüssige Phase - bestimmt dabei den Mechanismus der Relaxation. Während in der Gasphase zweifellos Stöße zwischen angeregtem Teilchen und Badgasmolekülen die Dynamik dominieren, existieren für VET in dichteren Phasen verschiedene Modellvorstellungen.

\subsubsection{VET in der Gasphase}

Generell ist der Schwingungsenergietransfer durch Stöße in der Gasphase in drei Kategorien einzuteilen: V-V-Transfer, V-R-Transfer und V-T-Transfer (V: Schwingung; R: Rotation; T: Translation). Handelt es sich bei dem Energieakzep- 
tor um ein Edelgas, findet nur V-T-Transfer statt. Landau und Teller formulierten erstmals den idealisierten Energietransfer von einem zweiatomigen schwingungsangeregten Molekül auf eine entlang der Molekülachse stoßende Punktmasse [27]. Sie konnten zeigen, daß der V-T-Transfer umso effizienter wird, je kleiner die reduzierte Masse $\mu$ des Stoßkomplexes und die Schwingungsfrequenz $\nu$ des Oszillators im Vergleich zur Stoßgeschwindigkeit ist. Letzteres ist besonders im Fall inelastischer Stöße gegeben. Eine quantenmechanische Beschreibung desselben Prozesses erfolgte durch Schwartz, Slawsky und Herzfeld (SSH-Theorie [28]), wobei die zentrale Aussage erhalten blieb, daß die Geschwindigkeit des Energietransfers von solchen Stößen bestimmt wird, die auf der Zeitskala der zu relaxierenden Schwingungsbewegung ablaufen.

Für aromatische Moleküle konnte experimentell gezeigt werden, daß der Stoßenergietransfer aus der niederfrequentesten Mode unter kleinstmöglicher Änderung der Schwingungsquantenzahl den bevorzugten Relaxationskanal darstellt [29]. Dieses Ergebnis wurde in SSH-Berechnungen, die für polyatomare Moleküle adaptiert wurden, bestätigt [30].

Die Geschwindigkeit der Umverteilung einer Anregungsenergie $E_{\mathrm{a}}$ ergibt sich aus dem Produkt einer gaskinetischen Stoßzahl mit der Relaxationswahrscheinlichkeit und der mittleren pro Stoß übertragenen Energie $\langle\Delta E\rangle$. Die Wechselwirkung zwischen angeregter Spezies und Badgas kann dabei beispielsweise mit einem Lennard-Jones-Potential beschrieben werden. $\langle\Delta E\rangle$-Werte sind für verschiedene Stoßgase experimentell über einen weiten Energiebereich (bis ca. $50000 \mathrm{~cm}^{-1}$ ) bestimmt worden, beispielsweise mit der KCSI-Methode [31]. Sie hängen von der Anregungsenergie und der Komplexität des Stoßpartners bzw. der Art des Energietransfers (V-V/V-R/V-T) ab.

\subsubsection{VET in dichten molekularen Umgebungen}

Das Konzept der intermolekularen Relaxation durch bimolekulare Stöße ist auch auf überkritische Lösungsmittel übertragbar. Schwarzer et al. konnten mit dem sog. IBC-Modell (isolated binary collisions) [32] den intermolekuren Energietransfer von Azulen an verschiedene überkritische Lösungsmitteln erfolgreich berechnen [33-35]. Unter Annahme unkorrelierter Einzelstöße zwischen Solvat und Solvens äußert sich die erhöhte Umgebungsdichte einzig in einer erhöhten Stoßzahl, während die Relaxationswahrscheinlichkeit und mittlere pro Stoß übertragene 
Energie konstant bleiben. Für eine detaillierte Beschreibung des Modells sei auf [36] verwiesen.

Auch in flüssiger Phase ist das IBC-Modell anwendbar, wenn eine geeignete Repräsentation des Solvat-Solvens-Wechselwirkungspotentials gefunden wird. Darüber hinaus haben sich noch zwei weitere Ansätze zur VET-Beschreibung in Lösung etabliert. Sie seien hier nur kurz erwähnt, da die in dieser Dissertationsschrift zusammengefaßten Untersuchungen größtenteils in der Gasphase stattfanden. Eine ausführlichere Übersicht ist z. B. in [13] dokumentiert.

- Störungstheoretische Erklärungsmodelle konzentrieren sich auf die fluktuierende Reibungskraft, die das Lösungsmittel auf den relaxierenden Oszillator mit Frequenz $\omega$ ausübt [32,37-39]. Die zentrale Größe dieser Theorien stellt die spektrale Dichte $G(\omega)$ des Lösungsmittels dar, welche der fouriertransformierten Autokorrelation der fluktuierenden Kraft entspricht. Der intermolekulare Energietransfer ist am effizientesten, wenn die dissipierte Energie der Frequenz $\omega_{\max }$ entspricht, bei der $G\left(\omega_{\max }\right)$ maximal ist. Da $\omega_{\max }$ typischerweise zwischen 10 und $100 \mathrm{~cm}^{-1}$ liegt, leisten niederfrequente Solvatmoden den Hauptanteil am VET-Prozess.

- Das Modell der Instantanen Normalmoden bietet einen alternativen Zugang zur Reibung des Lösungsmittels [40-42]. Diese Moden beinhalten die kollektiven Bewegungen der Lösungsmittelmoleküle und können (einfach angeregt oder als Obertöne) resonant Energie aus dem Oszillator des Solvats aufnehmen.

\subsubsection{IVR im nicht-isolierten Molekül}

In einer dichten molekularen Umgebung treten sowohl intra- als auch intermolekulare Energieumverteilung auf. Im Verlauf der Relaxation bleibt der Gesamtenergiegehalt des angeregten Moleküls nicht mehr wie im isolierten Fall konstant. Die Equilibrierung der Anregungsenergie beschränkt sich nicht mehr nur auf die $3 N-6$ Schwingungsfreiheitsgrade des Solvates, sondern bezieht die kollektiven Freiheitsgrade der Badmoleküle mit ein. Bei genügend großer Anzahl von Solvensmolekülen kühlt sich das System unter vollständiger Energiedissipation in endlicher Zeit auf die ursprüngliche thermische Besetzung ab. 


\section{Konkurrierende Prozesse}

Den Grad der Konkurrenz zwischen IVR und VET bestimmen u. a. der präparierte ZOBS sowie Dichte und spezifische Kopplungen des Lösungsmittels. Eine starke Konkurrenz bedeutet, daß Umverteilungs- und Transferkanäle parallel genutzt werden, d.h. daß der lokal angeregte Oszillator des Solvats gleichzeitig an das intra- und das intermolekulare Energiebad koppelt. Unter diesen Voraussetzungen gestalten sich mechanistische Studien der einzelnen Transferprozesse schwierig. In zeitaufgelösten Untersuchungen sind daher Bedingungen vorzuziehen, unter denen IVR und VET konsekutiv, d. h. auf unterschiedlichen Zeitskalen ablaufen.

Experimente in der Gasphase stellen den einfachsten Ansatz zur Zeitskalenseparierung dar. Hier werden die intermolekularen Wechselwirkungen und entsprechend VET durch Stöße moderiert. Bei niedrigen Gesamtdrücken und - wenn möglich - niedrigen Temperaturen ist der intermolekulare Transfer durch die stark verringerte Stoßfrequenz hinreichend verlangsamt, um das angeregte Molekül für die Dauer der intramolekularen Umverteilung als isoliert zu betrachten.

Eine zweite Möglichkeit zur Zeitskalenmanipulation bietet der helle Zustand selbst. Wie in den beiden vorhergehenden Abschnitten erläutert, sind intermolekulare Kopplungen besonders effizient, wenn der relaxierende Oszillator mit niedriger Frequenz schwingt und nur wenige Schwingungsquanten ausgetauscht werden. Dies gilt sowohl für die Gas- als auch für eine dichte Lösungsmittelphase. ${ }^{3}$ Aus einem hochfrequenten ZOBS wird die Anregungsenergie hingegen bevorzugt innerhalb des intramolekularen Energiebads umverteilt, da es die größere Anzahl Kopplungen niedriger Ordnung bereitstellt. Dementsprechend können IVR und VET als voneinander getrennt ablaufend aufgefaßt werden, wenn niederfrequente Moden erst im Zuge intramolekularer Relaxation aus hochfrequenten Moden bevölkert werden. In diese Kategorie fällt auch die im Rahmen der vorliegenden Arbeit untersuchte Energieumverteilung aus Obertönen von $\mathrm{CH}-S t r e c k s c h w i n g u n g e n$ mit $6000 \mathrm{~cm}^{-1}$. Daher ist eine vergleichende Interpretation der Pump-Probe-Messungen bei gasphasen- bzw. flüssigkeitsähnlichen Umgebungsdichten auf Basis desselben kinetischen Modells sequentieller Prozesse (s. Kap. 6) zulässig.

\footnotetext{
${ }^{3}$ In der Gasphase trifft dies im Falle gering schwingungsangeregter Spezies $\left(E_{\text {vib }} \lesssim\right.$ $\left.10000 \mathrm{~cm}^{-1}\right) \mathrm{zu}$.
} 
Kapitel 2 Grundlagen

\section{Rolle der molekularen Umgebung bei IVR}

Neben einer bloßen Konkurrenz von IVR und VET in dichten molekularen Umgebungen besteht darüber hinaus die Möglichkeit einer Beeinflussung der intramolekularen Dynamik durch das Lösungsmittel. Dieses Phänomen ist bis dato nicht vollständig verstanden und u. a. Thema dieser Arbeit. Prinzipiell sind zwei Arten der Beteiligung des Solvens am IVR des Solvats zu unterscheiden.

Bei einer indirekten Beeinflussung des IVR-Prozesses wirken die Lösungsmitteloder Badgasmoleküle in Form einer fluktuierenden Kraft auf den lokal angeregten Oszillator. Bimolekulare Stöße stören das relaxierende Molekül und verstärken die vorhandenen intramolekularen Kopplungen. Molekülspezifische Kopplungshierarchien blieben in diesem Fall erhalten, während die intramolekulare Umverteilung insgesamt beschleunigt würde. Die Art des Lösungsmittels/Badgases spielt eine untergeordnete Rolle, solange es sich um elastische Stöße handelt.

Eine direkte Beteiligung der molekularen Umgebung am Energiefluß ist ebenfalls denkbar. In diesem Fall würden Übergänge zwischen nicht-resonanten intramolekularen Schwingungszuständen durch Aufnahme und/oder Abgabe der entsprechenden Energiedifferenzen vom Lösungsmittel ermöglicht. In flüssiger Phase kämen als Akzeptormoden besonders Phononen mit Frequenzen von wenigen $\mathrm{cm}^{-1}$ in Frage, so daß hier eine Beschleunigung von Übergängen erfolgt, welche von vornherein nahezu resonant sind. In der Gasphase könnten je nach Natur des Stoßpartners auch größere Energieüberträge auftreten. Ein Erhalt des IVRMechanismus des isolierten Moleküls wäre dann nicht mehr gesichert. 


\section{Kapitel 3}

\section{Experimentelles}

Die Zielsetzung dieser Arbeit war es, den intramolekularen Schwingungsenergietransfer in aromatischen Molekülen zeitaufgelöst zu untersuchen. Als experimentelle Methode wurde die IR-Pump-UV-Probe-Spektroskopie gewählt, die sich für entsprechende Untersuchungen in der flüssigen Phase bereits bewährt hat $[13,43-$ 46]. Diese Methode konnte im Rahmen der vorliegenden Arbeit erstmals auch auf gasförmige Modellsysteme angewandt werden, wodurch globale Interpretationsansätze für die zugrundeliegenden Mechanismen des Energietransfers gefunden wurden, die vorher nicht zugänglich waren.

Dieses Kapitel widmet sich den experimentellen Details der durchgeführten Studien. Es werden zunächst das Prinzip der transienten Absorptionsspektroskopie sowie die daraus resultierende Observable erörtert. Hierauf folgt eine explizite Beschreibung der verwendeten Femtosekunden-Laserapparatur und der Signalaufnahme. Die gewünschten Meßbedingungen in der Gasphase oder in überkritischen Medien wurden mit Hilfe einer kombinierten Vakuum/Hochdruckapparatur realisiert, welche am Ende des Kapitels ebenfalls vorgestellt wird.

\subsection{Die IR-Pump-UV-Probe-Spektroskopie}

Sowohl die intra- als auch die intermolekulare Dynamik der Schwingungsenergieumverteilung in einem einzigen Experiment zu messen, setzt voraus, daß der Energiegehalt einzelner Schwingungen eines Moleküls zeitaufgelöst verfolgt werden kann. Eine simultane Detektion des Gesamtenergiegehalts aller Schwingungsfreiheitsgrade wäre wenig sinnvoll, da sich dieser idealerweise erst durch intermole- 


$$
\Delta t=0
$$
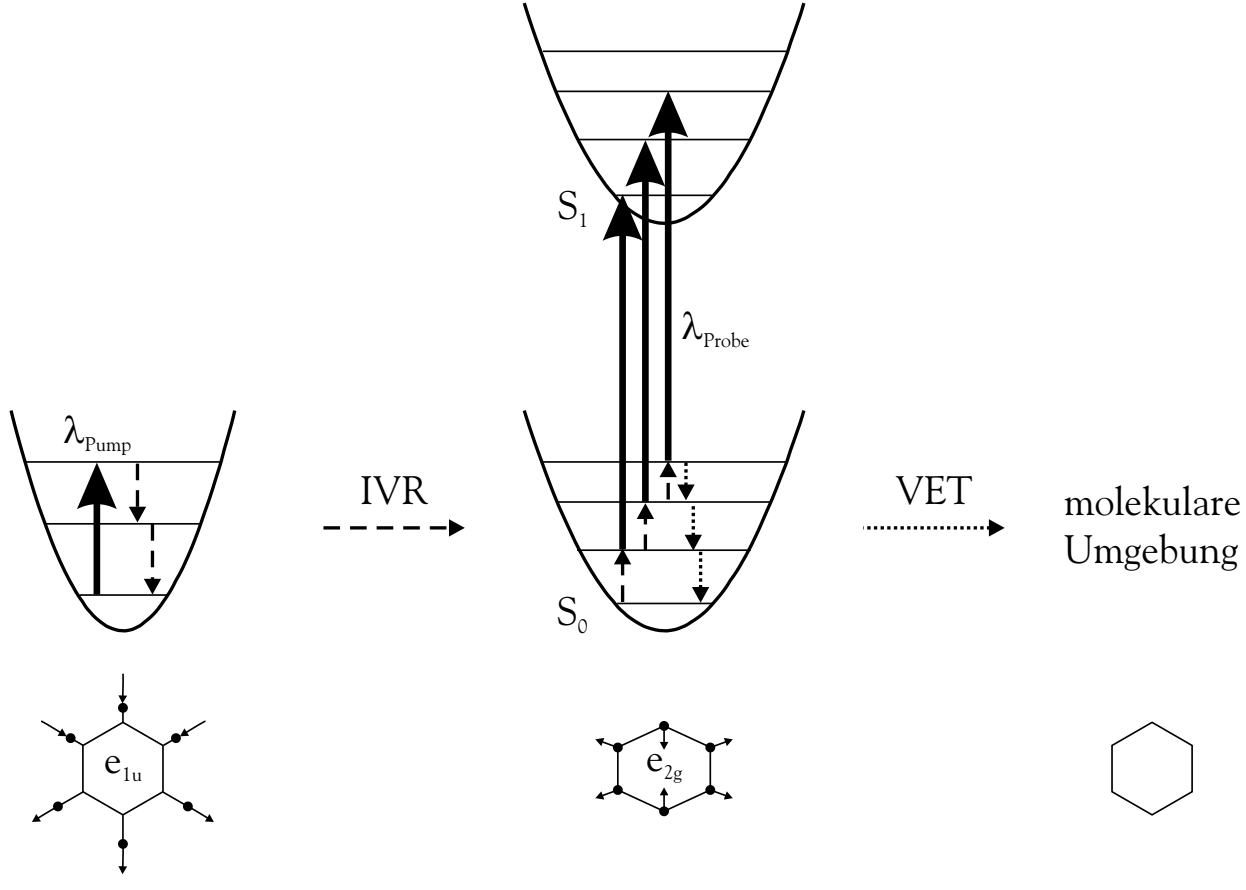

$\Delta t>0$

$\Delta t \gg 0$

Abbildung 3.1: Prinzip der IR-Pump-UV-Probe-Spektroskopie

kularen Energietransfer ändert, während der intramolekularen Umverteilung aber konstant bleibt.

Die fs-IR-Pump-UV-Probe-Spektroskopie bietet für geeignete Modellsysteme eine elegante Möglichkeit, beide Prozesse nahezu modenselektiv zu untersuchen. Das Prinzip dieser Methode soll anhand der Abb. 3.1 veranschaulicht werden. Beispielhaft ist hier der Pump-Probe-Vorgang am Benzol skizziert; dieses Prinzip ist aber aufgrund der Ähnlichkeit der Modellsysteme uneingeschränkt übertragbar.

Die Observable des Experiments ist die zeitliche Änderung der Absorption eines ultravioletten Probepulses, der den Franck-Condon-Übergang vom Grundzustand in den ersten angeregten elektronischen Zustand initiiert. Als $(0,0)$-Übergang ${ }^{1}$ ist

${ }^{1}$ Der Konvention entsprechend bezeichnet $(0,0)$-Übergang den Übergang zwischen den jeweiligen Schwingungsgrundzuständen. 
diese Anregung im Benzol streng symmetrieverboten. Wird aber gleichzeitig die Besetzung bestimmter Normalschwingungen geändert, entspricht die Gesamtsymmetrie des Übergangs der des elektrischen Übergangsdipolmomentes - der Übergang ist dann erlaubt. Diejenigen Normalschwingungen, die auf solche Weise elektronische Übergänge vermitteln, werden im folgenden als Franck-Condon(FC)aktiv bezeichnet. Wird die Energie des UV-Probepulses geringer als die $(0,0)$ Energiedifferenz zwischen $\mathrm{S}_{0}$ und $\mathrm{S}_{1}$ eingestellt, bestimmt die Population der heißen Banden FC-aktiver Moden im Grundzustand die Stärke der Absorption. Aufgrund der limitierten spektralen Breite des Probepulses werden nur Sequenzen der FC-aktiven Mode ${ }^{2}$ abgetastet. Hierbei nimmt die Absorption linear mit steigender Besetzung der Mode zu [47, 48].

Im Experiment wird nun mit einem Femtosekundenpuls im nahen Infrarotbereich eine definierte Anregungsenergie $\left(\tilde{\nu}=6000 \mathrm{~cm}^{-1}\right)$ im Molekül deponiert. Zum Zeitpunkt $t=0$ ist diese Energie im hellen Zustand nullter Ordnung, der in diesem Fall dem ersten Oberton $(v=2)$ der asymmetrischen $\mathrm{CH}-$ Streckschwingung mit $\mathrm{e}_{1 u^{-}}$Symmetrie entspricht, lokalisiert. In diesem Anfangszustand sind lediglich hochfrequente, nicht-FC-aktive Normalmoden populiert. Folglich ist das System für den UV-Probepuls transparent, wenn die zeitliche Verzögerung zum IR-Pumppuls $\Delta t=0$ beträgt.

Der präparierte helle Zustand mit lokalisierter Anregungsenergie gehört nicht zu den Eigenzuständen des Systems, weswegen eine intramolekulare Umverteilung der Energie (IVR, s. Abschn. 2.1) auf isoenergetische dunkle Zustände erfolgen muß. Somit wird die Anzahl an Schwingungsquanten in der asymmetrischen CH-Streckschwingung sukzessive abnehmen, in anderen Normalmoden dafür ansteigen. In Abb. 3.1 ist dies durch gestrichelte Pfeile angedeutet. Generell koppeln dunkle Zustände desto besser an den hellen Zustand, je kleiner die Summe transferierter Schwingungsquanten ist. Daher werden erst im späteren Verlauf des IVR zunehmend Schwingungszustände populiert, an denen eine niederfrequente FCaktive Mode, wie z. B. die Ringdeformationsschwingung mit $\mathrm{e}_{2 \mathrm{~g}}$-Symmetrie, mit einem oder mehreren Quanten beteiligt ist. Die Absorption des UV-Probepulses steigt deswegen mit zunehmender Verzögerung $\Delta t$ zum Pumppuls an, solange die intramolekulare Equilibrierung der Schwingungsenergie fortschreitet.

\footnotetext{
${ }^{2} \mathrm{Als}$ Sequenz werden Übergänge mit konstantem $\Delta \mathrm{v}$ bezeichnet. Im Gegensatz zu den Banden einer Progression sind die Übergänge einer Sequenz um nur wenige $\mathrm{cm}^{-1}$ gegeneinander verschoben.
} 


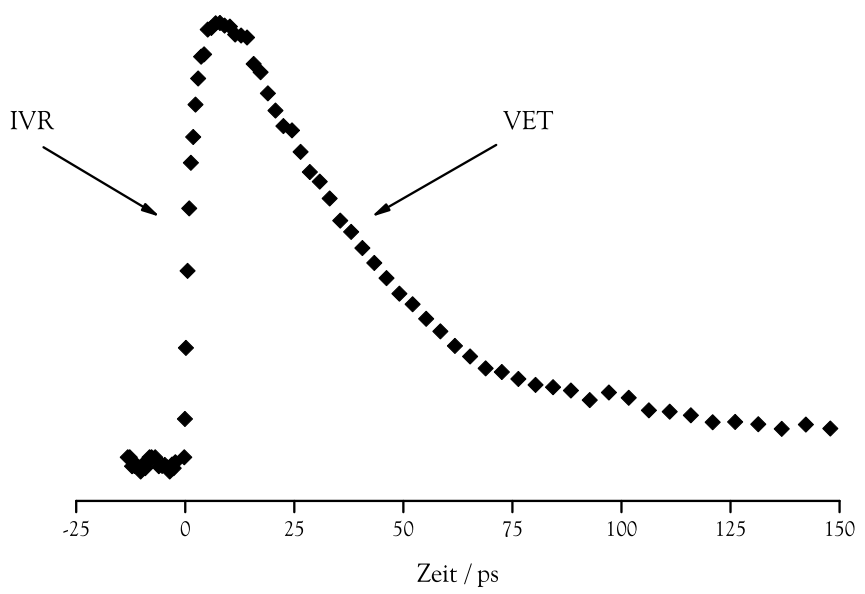

Abbildung 3.2: Typisches transientes Absorptionssignal bei flüssigkeitsähnlicher Umgebungsdichte

Im Falle des isolierten Moleküls endet die Umverteilung, wenn eine intramolekulare Equlibrierung der Anregungsenergie erreicht ist. In Anwesenheit einer molekularen Umgebung tritt jedoch mit fortschreitendem IVR verstärkt intermolekularer Energietransfer (VET, s. Abschn. 2.2) auf. Stöße mit kalten Molekülen führen Energie aus dem angeregten System ab. Diese Abkühlung bewirkt auch einen Rückgang der Population der FC-aktiven Mode, was in Abb. 3.1 durch gepunktete Pfeile dargestellt ist. Dementsprechend geht auch die Absorption des UV-Pulses für sehr große Verzögerungszeiten $(\Delta t \gg 0)$ auf Null zurück.

Aus der Auftragung der Änderung der optischen Dichte des Systems gegen die zeitliche Verzögerung zwischen Anregungs- und Abfragepuls resultiert somit ein transientes Absorptionssignal, welches gleichzeitig Information über intra- und intermolekularen Energietransfer enthält. Ein typisches Signal für flüssigkeitsähnliche Umgebungsdichten ist in Abb. 3.2 abgebildet. Auf die exakte Interpretation der transienten Absorption wird im Abschn. 6.1.1 ausführlicher eingegangen. An dieser Stelle sei nur erwähnt, daß unter Annahme eines im Vergleich zum VET schnellen IVR-Prozesses dem Signalanstieg die intramolekulare Dynamik entnommen werden kann, während der Signalabfall Aufschluß über die Geschwindigkeit des intermolekularen Transfers gibt. 


\subsection{Die Komponenten des Lasersystems}

Die Zeitskalen der intra- und intermolekularen Schwingungsdynamik, die im Rahmen dieser Dissertation untersucht wurden, reichen von hundert Femtosekunden (fs) bis zu einigen hundert Picosekunden (ps). Für die spektroskopische Untersuchung dieser Prozesse in der Zeitdomäne ist somit eine Femtosekundenzeitauflösung erforderlich. Die rasante Entwicklung der Lasertechnologie ermöglicht es inzwischen, mit kommerziell erhältlichen Systemen derart ultrakurze Pulse zu generieren. Optisch parametrische Verstärker erlauben es darüber hinaus, die Wellenlänge der Femtosekundenpulse über weite Bereiche durchzustimmen und so eine Vielzahl von verschiedenen molekularen Systemen mit der gleichen Methode zu untersuchen. Das durchstimmbare fs-Lasersystem, welches für diese Arbeit verwendet wurde, wird in den folgenden Abschnitten detailliert beschrieben. Das System ist in vier Komponenten aufgeteilt, den Hurricane ${ }^{\mathrm{TM}}$-Laser, die beiden optisch parametrischen Verstärker TOPAS und NOPA, sowie das Pump-ProbeSpektrometer. Abb. 3.3 zeigt die prinzipielle Verknüpfung dieser Module. Das Hurricane-System fungiert als Pumplaser der gesamten folgenden Apparatur. In den Verstärkern TOPAS und NOPA werden der infrarote Pumppuls bzw. der ultraviolette Probepuls generiert, die dann zur Messung in das Pump-ProbeSpektrometer eingekoppelt sind. Der Übersichtlichkeit halber wird jede Komponente in einem eigenen Abschnitt beschrieben. Neben den theoretischen Grund-

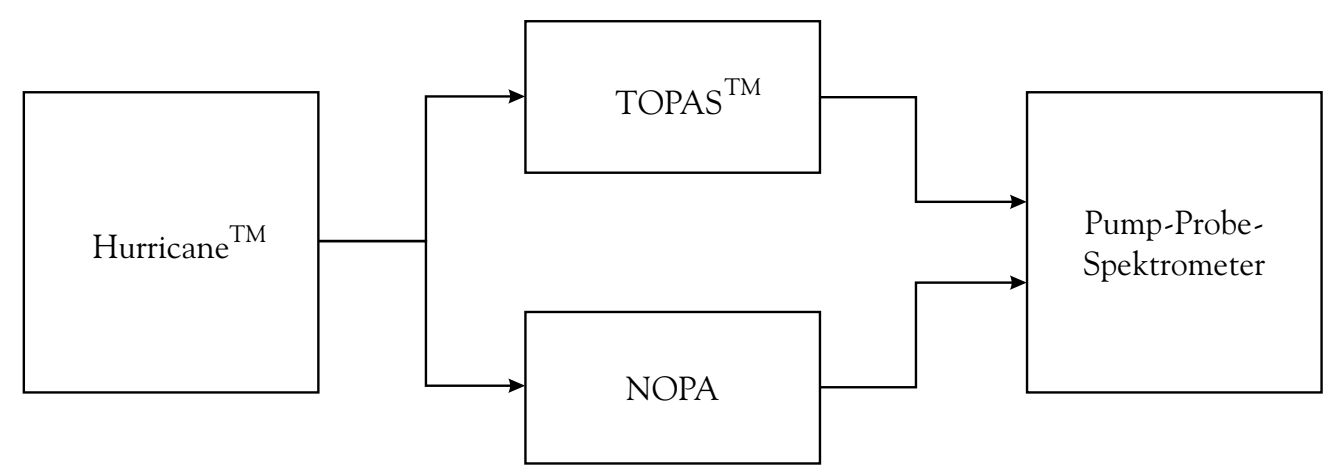

Abbildung 3.3: Prinzipieller Aufbau des Lasersystems 
lagen des jeweils zugrundeliegenden physikalischen Prozesses werden der optische Aufbau, die charakteristischen Pulseigenschaften und die tägliche Justierung erörtert.

\subsubsection{Das Hurricane ${ }^{T M}-$ System}

Das Hurricane ${ }^{\mathrm{TM}}$-Lasersystem (Spectra-Physics) generiert die für die ultraschnelle Spektroskopie erforderlichen Femtosekundenpulse. Es vereint in einem kompakten Aufbau die vier typischen Komponenten eines Kurzpuls-Festkörperlasers: Oszillator, Strecker, regenerativen Verstärker (regenerative amplifier, RA) und Kompressor. Der optische Aufbau der Hurricane-Einheit ist in Abb. 3.4 skizziert. Da es sich um ein abgeschlossenes, vollständig temperierbares System handelt, ist es einem selbstgebauten fs-Laser in puncto Aufwärmzeit, Stabilität und Justierungsbedarf deutlich überlegen. Im folgenden wird eine kurze Einführung in die Erzeugung und Verstärkung der ultrakurzen Laserpulse im Hurricane ${ }^{\mathrm{TM}}$ gegeben. Für eine detailliertere Beschreibung der einzelnen Komponenten inklusive Justierungsanweisungen sei auf die entsprechenden Handbücher [49-51] verwiesen.

\section{Die Erzeugung ultrakurzer Pulse}

Für die zeitaufgelöste Spektroskopie hat die Erfindung gepulster Laser einen großen Fortschritt bedeutet. Durch die Q-Switch-Technik können mit nahezu jedem Laser kurze Pulse mit bis zu Nanosekundendauer realisiert werden. Um noch kürzere Pulse zu erzeugen, wird meistens die Technik der Modenkopplung (mode locking, ml) angewandt. Bei großer Bandbreite des Laserübergangs oszilliert im Resonator der Länge $L$ eine Vielzahl von longitudinalen Moden simultan, deren Frequenzabstand für benachbarte Moden

$$
\Delta \nu=\frac{c}{2 L}
$$

beträgt. Stehen diese Moden in einer festen Phasenbeziehung zueinander, werden sie als gekoppelt bezeichnet. Durch Interferenz der gekoppelten Moden wird die Laserstrahlung als ein kurzer Puls der Halbwertsdauer

$$
\Delta \tau_{P}=\frac{a}{\Delta \nu}
$$

emittiert, der im Resonator umläuft. Voraussetzung hierfür ist selbstverständlich ein Lasermedium mit breitem Emissionsvermögen im Laserübergang, da Pulsdau- 


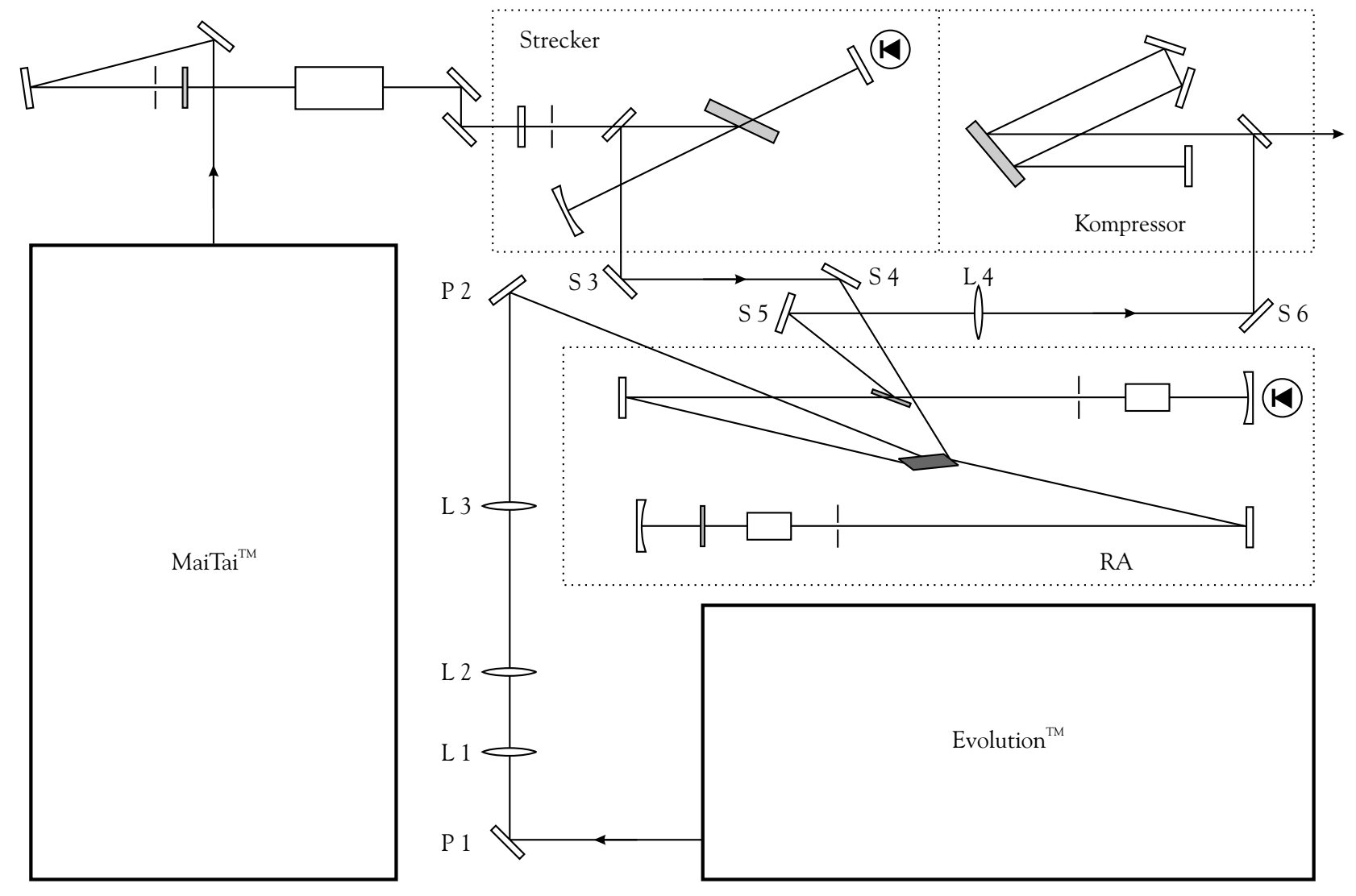

Abbildung 3.4: Optischer Aufbau des Hurricane ${ }^{\mathrm{TM}}-$ Systems 
er und spektrale Breite aufgrund der Heisenbergschen Unschärferelation invers proportional zu einander sind. Festkörperlaser wie der Titan-Saphir(Ti:Sa)-Laser sind hierfür besonders geeignet. Der Proportionalitätsfaktor $a$ der Bandbreite hängt von der Form des Linienprofils ab. Für gaussförmige Pulse gilt

$$
a=\frac{2 \ln 2}{\pi}=0.441
$$

Dies stellt eine untere Grenze dar, anhand derer die Güte der Modenkopplung überprüft werden kann.

Technisch kann die Modenkopplung auf drei Arten realisiert werden: aktiv, passiv oder durch synchrones Pumpen. Aktive Modenkopplung wird durch einen im Resonator positionierten Modulator erreicht, welcher die Verluste im Resonator mit der Frequenz sinusförmig moduliert, die gerade dem Frequenzabstand $\Delta \nu$ benachbarter Resonatormoden entspricht. Als Modulatoren können elektrooptische (Pockelszellen) oder akustooptische Elemente eingesetzt werden. Passive Modenkopplung wird durch einen sättigbaren Absorber innerhalb des Resonators erreicht, der ebenfalls eine Verlustmodulation hervorruft. Diese beruht auf der intensitätsabhängigen Absorption des Absorbers. Ein Spezialfall der passiven Modenkopplung stellt das Kerr-Lens-Modelocking dar, wobei das Lasermedium selbst als Absorber fungiert. Aufgrund des intensitätsabhängigen Brechungsindex des Mediums wird eine Linse ausgebildet, welche zu einem besseren Überlapp von Pumplicht und zu verstärkender Lasermode führt.

\section{Der MaiTai}

Im MaiTai-Modul findet die Erzeugung der modengekoppelten fs-Pulse statt. Das Modul besteht aus zwei getrennten Laserkammern, von denen eine den cwPumplaser, die andere den Ti:Sa-Oszillator enthält. Eine Abbildung des patentierten Systems kann an dieser Stelle leider nicht gezeigt werden.

Der Pumplaser ist ein diodengepumpter Nd:YAG-Laser, dessen Zentralwellenlänge bei $1064 \mathrm{~nm}$ liegt. Innerhalb der Lasercavity befindet sich ein phasenangepasster LBO-Kristall, der diese Frequenz mit hoher Konversionseffizienz verdoppelt. Somit liegt ein sehr stabiles kontinuierliches Pumplicht der Wellenlänge $532 \mathrm{~nm}$ bei einer konstanten Leistung von $5 \mathrm{~W}$ vor, das in die zweite Laserkammer eingekoppelt wird.

In dieser Kammer befindet sich der eigentliche fs-Laser, ein Ti:Saphir-Oszillator 
in gefalteter Geometrie. Dieser wird longitudinal gepumpt, d.h. das Pumplicht wird zu einer schmalen Linie innerhalb des Kristalls fokussiert, deren Durchmesser der zu verstärkenden $\mathrm{TEM}_{00}$-Lasermode angepafst ist.

Die Phasenkopplung der Ti:Sa-Pulse wird aktiv durch einen innerhalb des Resonators befindlichen akustooptischen Modulator erreicht. Die Länge des Resonators resultiert in einer Umlaufzeit des ml-Pulses von ca. $12.8 \mathrm{~ns}$, woraus sich eine eine Repetitionsrate von etwa $80 \mathrm{MHz}$ ergibt.

Die doppelbrechende Eigenschaft des Ti:Sa-Lasermediums erlaubt es, durch Anpassen des Kristallwinkels die Emissionswellenlänge über einen Bereich von 750$850 \mathrm{~nm}$ durchzustimmen. In diesem Fall wurde die Zentralwellenlänge auf $800 \mathrm{~nm}$ eingestellt. Ein Prismenpaar innerhalb des Resonators kompensiert die in den optischen Komponenten auftretende positive Gruppengeschwindigkeitsdispersion und die im Lasermedium induzierte Selbst-Phasenmodulation.

Eine Justierung des MaiTai durch den Benutzer ist weder möglich noch nötig, da Leistung und Stabilität über interne Photodioden überwacht werden, welche direkt an die Diodensteuerung gekoppelt sind. Die Steuerung des gesamten Systems erfolgt über ein Computerinterface.

\section{Der Strecker}

Festkörperlaser wie der Ti:Sa-Laser erlauben es dank ihrer starken Sättigungsfluoreszenz, relativ hohe Pulsenergien aus vergleichsweise kompakten Laseraufbauten zu gewinnen. Darüber hinaus verfügt Ti:Saphir über einen sehr hohen Wirkungsgrad, der für die Verstärkung von sub-Pikosekundenpulsen nötig ist. Allerdings kann es bei zu hohen Spitzenintensitäten zur Selbstfokussierung und damit zur Schädigung des Kristalls kommen. Daher ist es nötig, die Intensität in Verstärkerkristallen auf weniger als $10 \mathrm{GW} / \mathrm{cm}^{2}$ zu beschränken.

Eine Lösung dieses Problems stellt die Verstärkung gechirpter Pulse dar. Die Beziehung zwischen Pulsdauer und spektraler Bandbreite fordert, daß ultrakurze Pulse ein sehr breites Spektrum aufweisen. Solche Pulse können zeitlich gestreckt und ihre Spitzenintensität verringert werden, wenn ihre einzelnen Frequenzen gegeneinander verzögert werden. Hierfür bietet es sich an, die dispersiven Eigenschaften von Prismen oder Gittern auszunutzen. Diese sind in der Lage, durch Verzögerung der blauen spektralen Anteile gegenüber den roten eine positive Gruppengeschwindigkeitsdispersion (group velocity dispersion, GVD, auch positiver Chirp genannt) zu induzieren und den Puls zeitlich zu strecken. Entsprechend 


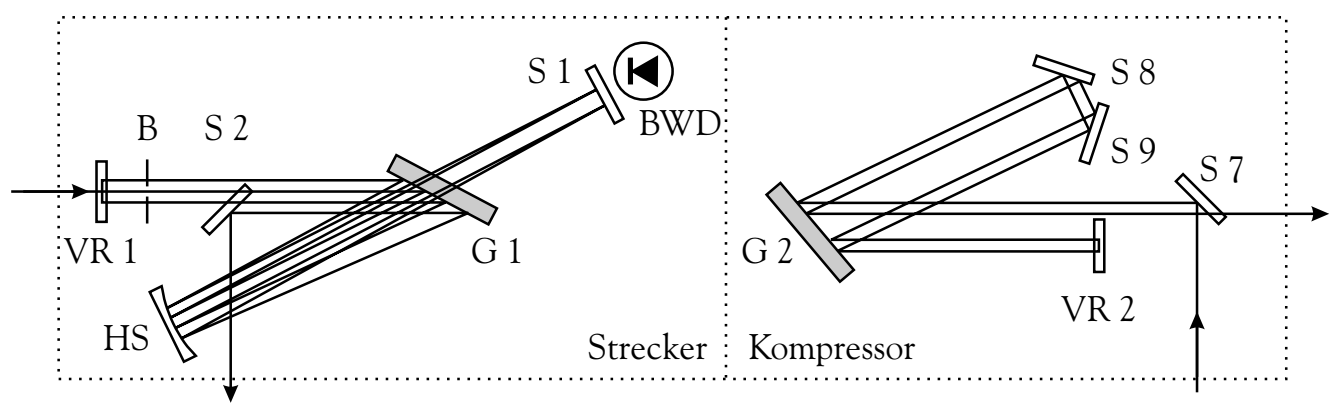

Abbildung 3.5: Optischer Aufbau des Streckers und des Kompressors in Detailansicht (Erläuterung der Abkürzungen siehe Text)

kann durch Komponenten, die einen negativen Chirp induzieren, der gestreckte Puls wieder komprimiert werden.

Im Hurricane ${ }^{\text {TM}}$-Strecker, dargestellt in der linken Hälfte von Abb. 3.5 wird dieses Prinzip in einer gefalteten Streckergeometrie mit einem Gitter (G1) und einem Goldhohlspiegel (HS) angewandt. Die Anordnung mit vertikalem Retroreflektor (VR1) und Faltspiegel (S1) bewirkt, daß die Pulse insgesamt viermal am Gitter gebeugt werden. Dabei erzeugt der Goldspiegel sowohl einen Strahlversatz, der die Auskopplung mittels des Spiegels S2 erleichtert, als auch die gewünschte positive GVD. Eine hinter S1 befindliche Photodiode (BWD) überwacht die Bandbreite des räumlich und zeitlich gestreckten Spektrums.

\section{Der regenerative Verstärker}

In der regenerativen Verstärkereinheit (RA) wird jeweils ein gestreckter Puls selektiert und maximal verstärkt. Die zusätzliche Energie wird ihm in einem zweiten Titan-Saphir-Kristall durch den Evolution ${ }^{\text {TM}}$-Pumplaser zugeführt.

Der Evolution $^{\mathrm{TM}}$ ist ein diodengepumpter, innerhalb der Lasercavity verdoppelter Nd:YLF-Laser, der mittels Q-Switch ca. 50 ns lange Pulse mit einer Zentralwellenlänge von $527 \mathrm{~nm}$ bei ca. $8 \mathrm{~W}$ und einer Repetitionsrate von $1 \mathrm{kHz}$ generiert. Diese werden im Teleskop (L1 und L2, Abb. 3.4) kollimiert, von L3 fokussiert und über die Pumpspiegel P1 und P2 (Abb. 3.4) in den RA eingekoppelt. 


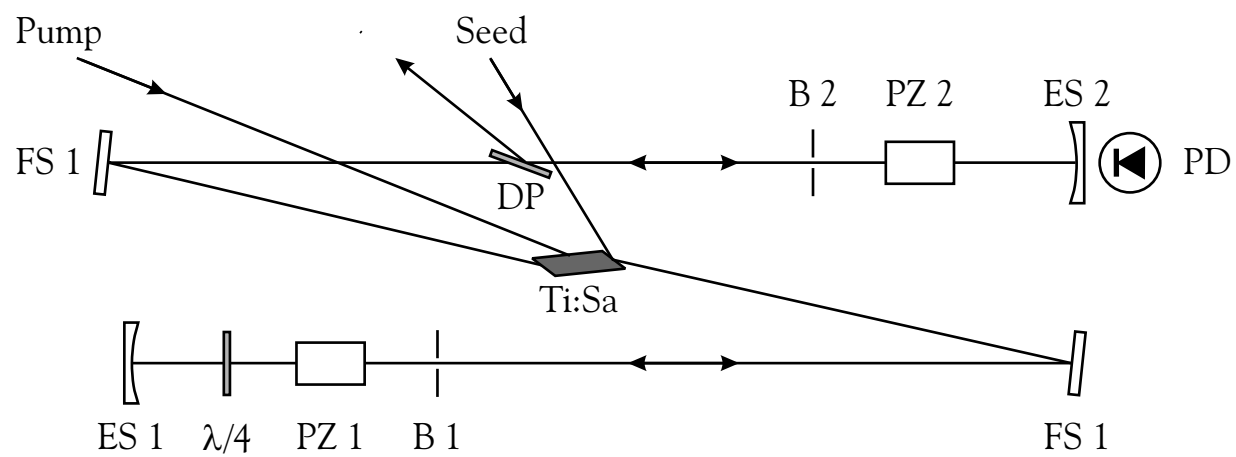

Abbildung 3.6: Optischer Aufbau des regenerativen Verstärkers (RA) in Detailansicht (Erläuterung der Abkürzungen siehe Text)

Der RA selber ist ein Verstärkermodul, dessen Funktionsweise anhand der Detailabbildung 3.6 veranschaulicht werden kann. Die modengekoppelten, zeitlich gestreckten, p-polarisierten Seed-Pulse aus dem MaiTai werden in den RA eingekoppelt, indem sie an der Endfläche des Titan-Saphir-Verstärkerkristalls (Ti:Sa) in Richtung des Faltspiegels FS1 reflektiert werden. Solange die EinkoppelPockelszelle (PZ1) deaktiviert ist, durchlaufen die Pulse den unteren Resonatorarm des RA einmal und verlassen ihn unverstärkt. Wird aber der Evolution ${ }^{\mathrm{TM}}$-QSwitch geöffnet und ein Pumppuls auf den Ti:Sa-Kristall gegeben, aktiviert dies PZ1, um den Verstärkungsprozess zu beginnen. Wieder wird der zu verstärkende Puls durch Reflektion am Ti:Sa-Kristall eingekoppelt und von FS1 reflektiert. Er durchläuft die noch ausgeschaltete PZ1 und das $\lambda / 4$-Plättchen. Der Endspiegel ES1 reflektiert den Puls in sich zurück. Dieser hat nun das $\lambda / 4$-Plättchen zweimal passiert und somit s-Polarisation, daher wird er nun vom Ti:Sa-Kristall und den anderen Resonatoroptiken transmittiert. Sobald der Puls aus PZ1 ausgetreten ist, wird diese durch Anlegen einer Spannung von $3500 \mathrm{~V}$ als $\lambda / 4$-Platte geschaltet, die die Wirkung des $\lambda / 4$-Plättchens dahinter aufhebt und den Puls somit im Resonator fängt. Nach ca. sechzehn Resonatorumläufen ist die Pulsverstärkung maximal (ca. 106-fach). Nun wird die Auskoppel-Pockelszelle (PZ2) aktiviert, die nach zweimaligem Passieren die Polarisation des Pulses um insgesamt $90^{\circ}$ dreht. 
Kapitel 3 Experimentelles

Somit kann er nun über den Dünnschichtpolarisator (DP) aus dem Resonator ausgekoppelt werden. Über die Spiegel S5 und S6 (Abb. 3.4) wird der Strahl schließlich in den Kompressor gelenkt. Eine hinter ES2 angebrachte Photodiode (PD) ermöglicht es, die Güte der Resonatorjustage zu überprüfen sowie den Moment der Auskopplung des verstärkten Pulses zu wählen. Es empfiehlt sich, die Justage des RA-Resonators alle vier Wochen durch Anpassung von ES1 und ES2 auf die Aperturen B1 bzw. B2 zu optimieren.

\section{Der Kompressor}

Ebenso wie der Strecker weist auch der Kompressor im Hurricane ${ }^{\mathrm{TM}}$ dank gefalteter Geometrie eine sehr kompakte Bauweise auf. In der rechten Hälfte von Abb. 3.5 ist der Strahlengang dieser Einheit skizziert. Die verstärkten Pulse werden über S7 eingekoppelt. Mittels des horizontalen (HR) und des vertikalen Retroreflektors (VR2) werden sie viermal auf dem Gitter (G2) abgebildet, das einen negativen Chirp induziert und so die Pulse auf ca. $100 \mathrm{fs}$ komprimiert. Der horizontale Retroreflektor (S8, S9) befindet sich auf einer elektronisch gesteuerten Translationsbühne, so daß die Kompressorlänge für optimale Pulsdauern über eine Fernsteuerung angepaßt werden kann, ohne das System zu öffnen und es schwankenden Umweltbedingungen auszusetzen. Mittels eines Strahlteilers wird die Ausgangsleistung des Hurricanesystems auf die Verstärkermodule TOPAS $(80 \%)$ und NOPA (20\%) aufgeteilt. Die komprimierten Laserpulse haben bei Verlassen des Hurricane ${ }^{\mathrm{TM}}$ eine Zentralwellenlänge von $800 \mathrm{~nm}$. Die Energie beträgt ca. $1 \mathrm{~mJ}$ pro Puls bei einer Repetitionsrate von $1 \mathrm{kHz}$. Abb. 3.7 stellt Spektrum und Autokorrelation der Hurricanepulse dar. Die spektrale Breite der Pulse beträgt $\Delta \lambda=11 \mathrm{~nm}$. Die Pulsdauer kann der Autokorrelation entnommen werden. Für gaufförmige Zeitprofile ist die Breite auf halber Höhe des Maximums des einzelnen Pulses $\Delta \tau_{\text {deconv. }}$ (deconvolution, engl. Entfaltung) um einen Faktor $\sqrt{2}$ kleiner als die der Korrelation. Die Hurricane-Pulse haben somit eine Länge von 85 fs und sind mit $\Delta \tau \cdot \Delta \nu=0.42$ nahezu bandbreitebegrenzt, was für eine sehr effiziente Modenkopplung spricht. Die Flanken der Autokorralation zeigen leichte Schultern, welche durch Optimierung des Kompressorgitterwinkels und der Kompressorlänge minimiert wurden und die Qualität des Experiments nicht weiter beeinflussen. 

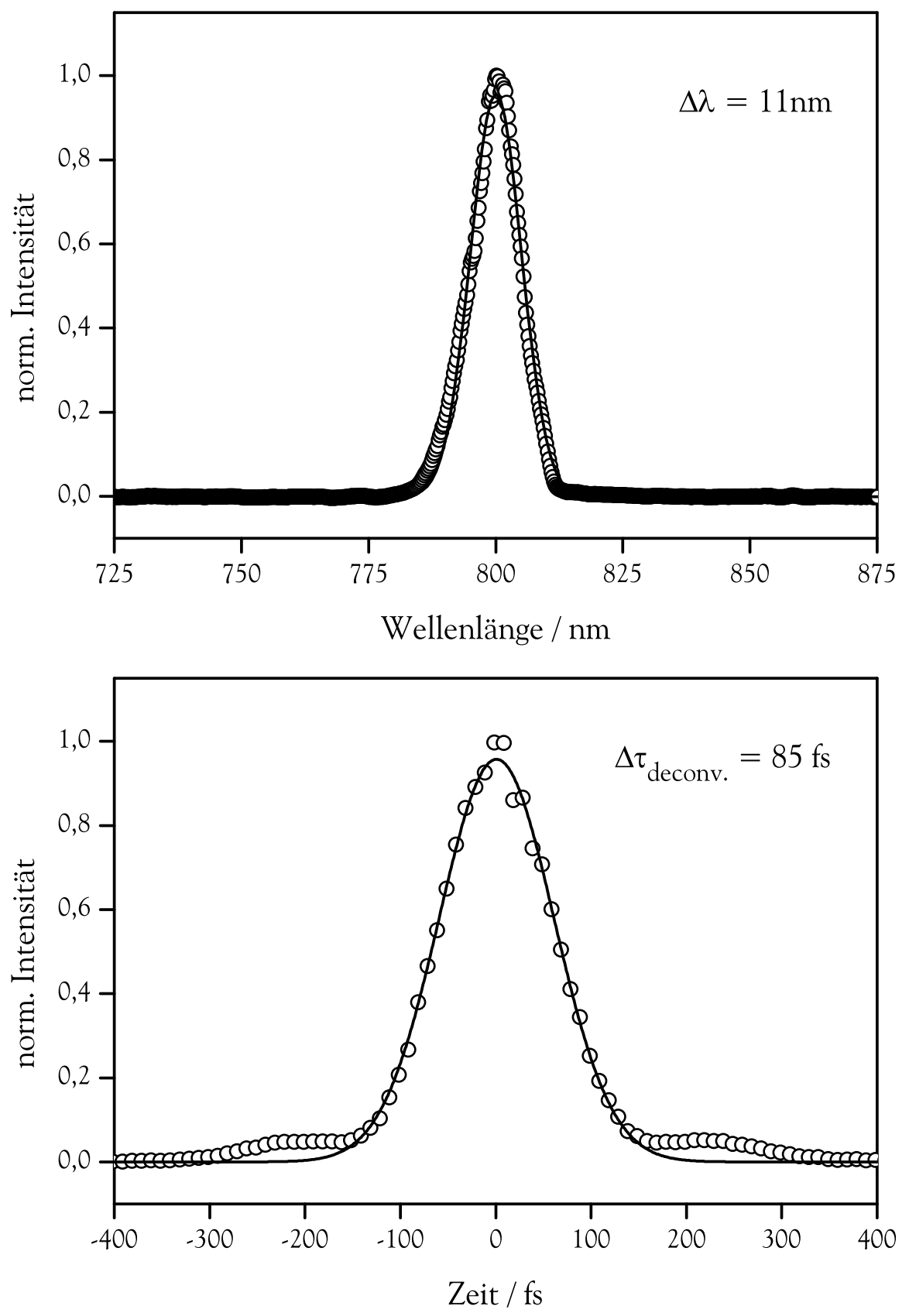

Abbildung 3.7: Spektrum (oben) und Autokorrelation (unten) des Hurricane ${ }^{\mathrm{TM}}$ 


\subsubsection{Das NOPA-System: Erzeugung von UV-Pulsen}

Der NOPA (Noncollinear Optical Parametric Amplifier) stellt einen der beiden optisch-parametrischen Verstärker im Aufbau des Experiments dar. Er dient der Erzeugung eines fs-Pulses mit einer Wellenlänge im UV- und VIS-Bereich von 250-700 nm, der anschließend (siehe Abschnitt 3.2.4) zum UV-Probepuls frequenzverdoppelt wird. Das zugrundeliegende Prinzip der optisch-parametrischen Verstärkung findet auch im TOPAS (siehe Abschnitt 3.2.3) Anwendung und wird daher zunächst in allgemeiner Form beschrieben. Anschließend wird die Adaption dieses Prinzips auf die speziellen Anforderungen der fs-VIS-Photonen-Erzeugung im NOPA dargelegt, bevor der zweistufige optische Aufbau selbst beschrieben wird.

\section{Optisch-parametrische Verstärkung}

Die optische Verstärkung von monochromatischem Laserlicht gehört zu den DreiWellen-Wechselwirkungen. Eine schwache Signal-Welle der Frequenz $\omega_{\mathrm{S}}$ (auch seed genannt) wird in einem nichtlinearen optischen Kristall (z. B. BBO) mit einer starken Pump-Welle der Frequenz $\omega_{\mathrm{P}}$ überlagert. Bei gegebener Einstellung des Kristallwinkels sind die Phasengeschwindigkeiten von Signal und Pump aufeinander angepaßst, und es entsteht eine dritte Lichtwelle der Frequenz $\omega_{\mathrm{I}}$, die als Idler bezeichnet wird. Die Energie bleibt hierbei erhalten, es gilt somit

$$
\omega_{\mathrm{I}}=\omega_{\mathrm{P}}-\omega_{\mathrm{S}}
$$

Desweiteren führt der Impulserhalt zur zweiten Bedingung, daß für phasenangepaßte Wellen mit Wellenvektor $k_{i}$

$$
\Delta k=k_{\mathrm{S}}+k_{\mathrm{I}}-k_{\mathrm{P}}=0
$$

ist. Am Beginn des nichtlinearen Mediums wird $\omega_{\mathrm{S}}$ somit zunächst nur wenig verstärkt, während die Idler-Welle mit $\omega_{\text {I }}$ neu entsteht. Je weiter die Wellen durch den Kristall laufen, desto mehr nimmt die Verstärkung des Signals zu.

In der optisch-parametrischen Verstärkung kann die Wellenlänge von Signalund Idlerwelle durch Anpassung der experimentellen Bedingungen variiert werden. Gleichung (3.4) lautet in diesem Fall

$$
n_{\mathrm{I}} \omega_{\mathrm{I}}=n_{\mathrm{P}} \omega_{\mathrm{P}}-n_{\mathrm{S}} \omega_{\mathrm{S}}
$$


Die Parameter $n_{i}$ hängen empfindlich von der Orientierung des nichtlinearen Kristalls, der Temperatur, dem Druck und/oder einem angelegten elektrischen Feld ab. Oft sind die Frequenzen von Signal- und Idlerwelle so kontinuierlich über einen weiten Bereich durchstimmbar.

\section{Nichtkollineare optisch-parametrische Verstärkung und Superfluoreszenz}

Die Phasenanpassung wird in herkömmlichen Verstärkern meist durch Rotation des Kristalls relativ zur optischen Achse erreicht, auf der die Signal-, Pumpund Idlerwellen kollinear liegen. Allerdings führt dies dazu, daßs die drei Gruppengeschwindigkeiten unterschiedlich sind, wodurch die Pulse mit verschiedenen Geschwindigkeiten durch den Kristall wandern und Signal- und Idlerpuls zeitlich stark verlängert werden, weil die neuen Photonen räumlich hinter der zu verstärkenden Pulsfront generiert werden. Gale et al. [52] zeigten, daß in einem nichtkollinearen Aufbau dieses Problem umgangen werden kann. Für einen Winkel $\theta$ zwischen Seed- und Pumpstrahl ergibt sich ein Winkel $\gamma$ zwischen Seed- und Idlerstrahl, der näherungsweise gemäß

$$
\gamma \approx \theta \cdot\left(1+\frac{\lambda_{\mathrm{I}}}{\lambda_{\mathrm{S}}}\right)
$$

festgelegt ist. Die Gruppengeschwindigkeit des Idlers ist i. A. größer als die des Signals. Es existiert somit ein Winkel $\gamma$, unter dem die Projektion der IdlerGruppengeschwindigkeit $v_{\mathrm{I}}$ auf den Signal-Wellenvektor $k_{\mathrm{S}}$ genau der SignalGruppengeschwindigkeit $v_{\mathrm{S}}$ entspricht, daß also gilt

$$
v_{\mathrm{I}} \cdot \cos \gamma=v_{\mathrm{S}} .
$$

Die Signal-Photonen, die aus der Verstärkung des Idlers resultieren, entstehen so am gleichen Ort wie die aus der Verstärkung des ursprünglichen Signals hervorgehenden, die Pulse werden nicht verlängert.

Im klassischen optischen Bild kann die parametrische Verstärkung nur stattfinden, wenn bei Eintritt in das nichtlineare Medium neben der Pumpwellenlänge noch mindestens eine andere Wellenlänge (Signal oder Idler) vorhanden ist. Mit einer ausreichend hohen Pumpenergie allerdings werden Signal und Idler auch dann generiert, wenn die Zahl der Photonen mit $\omega_{S}$ und $\omega_{I}$ vorher Null war. Dieses Phänomen ist als optische parametrische Superfluoreszenz bekannt. Es kann quantenmechanisch als parametrische Verstärkung der Nullpunktsfluktuationen 

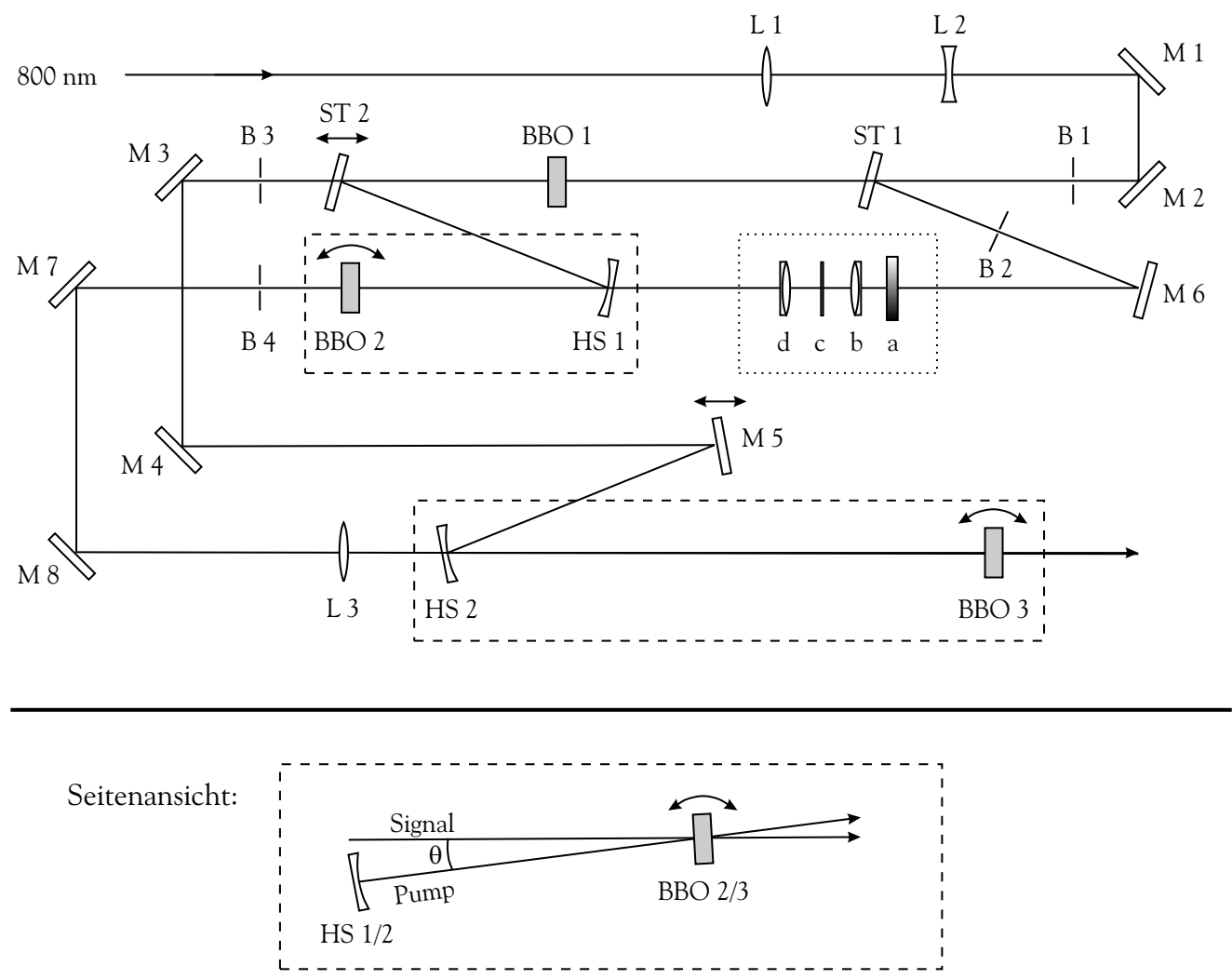

Abbildung 3.8: Optischer Aufbau des NOPA-Systems

des elektrischen Felds um die Gitterpunkte des Kristalls verstanden werden [53]. Im Falle des NOPA kann die Güte der Konversion daran gemessen werden, wieviel superfluoreszente Strahlung in Anwesenheit des Seed-Pulses in verstärkte Strahlung umgewandelt wird.

\section{Der optische Aufbau}

Der in dieser Arbeit verwendete NOPA ist zweistufig aufgebaut. Der ersten Stufe dient ein Weißlichtkontinuum mit positivem Chirp als Seedwelle, aus dem durch Anpassung des zeitlichen Überlapps mit dem Pumppuls jede beliebige Wellenlänge selektiv verstärkt werden kann. Hierbei wird in der ersten Stufe die parametrische Strahlung in niedriger Intensität, aber hoher Qualität des Spektrums 
und des räumlichen Pulsprofils generiert. Dieses Licht dient als Seed-Welle der zweiten Stufe, wo seine Intensität um ein Vielfaches verstärkt wird.

Der optische Aufbau ist in Abb. 3.8 skizziert. Das NOPA-System wird mit $20 \%$ der aus dem Hurricane ${ }^{\mathrm{TM}}$ ausgekoppelten Ti:Sa-Fundamentalen bei $800 \mathrm{~nm}$ gepumpt. Am Eingang des NOPA wird der Strahldurchmesser mit Hilfe eines Teleskops (L1 und L2) von 9 auf $3 \mathrm{~mm}$ reduziert. Die Anordnung der Linsen ist so gewählt, daß eine leichte Fokussierung des Strahls auf den Kristall BBO1 $\left(7 \times 7 x 1 \mathrm{~mm}^{3}\right.$, Typ I Phasenanpassung $\left.\left(30^{\circ}\right)\right)$ erfolgt. Der Strahlengang ist durch Justage der Spiegel M1 und M2 auf die Blenden B1 bzw. B2 auf eine Höhe von $12 \mathrm{~cm}$ über der Tischoberfläche festgelegt. Der Hauptanteil der Ti:SaFundamentalen passiert den Quarzstrahlteiler ST1 und erzeugt im Kristall BBO1 den Pumppuls mit $400 \mathrm{~nm}$ Wellenlänge. Am beweglichen Strahlteiler ST2 wird dieser auf die erste und zweite NOPA-Stufe im Verhältnis 30:70 aufgeteilt.

$4 \%$ der Ti:Sa-Fundamentalen werden an ST1 zur Kontinuumserzeugung abgeteilt. Der Strahlteiler ist keilförmig geschliffen, wodurch die unerwünschte Rückseitenreflektion räumlich leichter abgetrennt werden kann. Die Vorderseitenrefklektion wird mittels der Blende B2 im Durchmesser auf $1 \mathrm{~mm}$ reduziert und über den Spiegel M6 in die Anordnung zur Kontinuumserzeugung (gepunktete Linie in Abb. 3.8) eingekoppelt. Diese besteht aus einem stufenlos anpaßbaren Graufilter (a), einer Achromat-Linse (b), die das intensitätsangepaßte Licht in eine $1 \mathrm{~mm}$ dicke Saphirplanplatte (c) fokussiert. Die Saphirplatte ist so geschliffen, daß die Kristallachse parallel zur optischen Achse verläuft. Unter diesen Bedingungen kann durch stimulierte Ramanübergänge im Kristall ein zentrosymmetrisches Weißlichtkontinuum erzeugt werden, welches mittels einer zweiten Achromatlinse (d) so fokussiert wird, daß die Strahltaille wenige Millimeter vor dem Kristall BBO2 (5x5x1 mm $\mathrm{mm}^{3}$, Typ I Phasenanpassung $\left.\left(32,5^{\circ}\right)\right)$ liegt. Dabei verläuft der Strahl oberhalb des Hohlspiegels HS1 $(r=120 \mathrm{~mm})$, welcher den an ST2 reflektierten Pumpstrahl auf BBO2 bündelt. Der Abstand des Kristalls zum Pumpfokus ist so gewählt, daß mit bloßem Auge im völlig abgedunkelten Labor gerade noch der Superfluoreszenzring wahrgenommen werden kann. Durch Anpassung der zeitlichen Verzögerung (Translation von ST2), des Winkels $\theta$ zwischen Pumpstrahl und Weißlichtkontinuum sowie des Kristallwinkels für optimale Phasenanpassung wird ein zeitlicher und räumlicher Überlapp im Kristall realisiert, der die Generierung einer schwachen Signalwelle im sichtbaren Frequenzbereich bedingt. Die Blende B4 trennt die verbleibende Superfluoreszenz sowie 

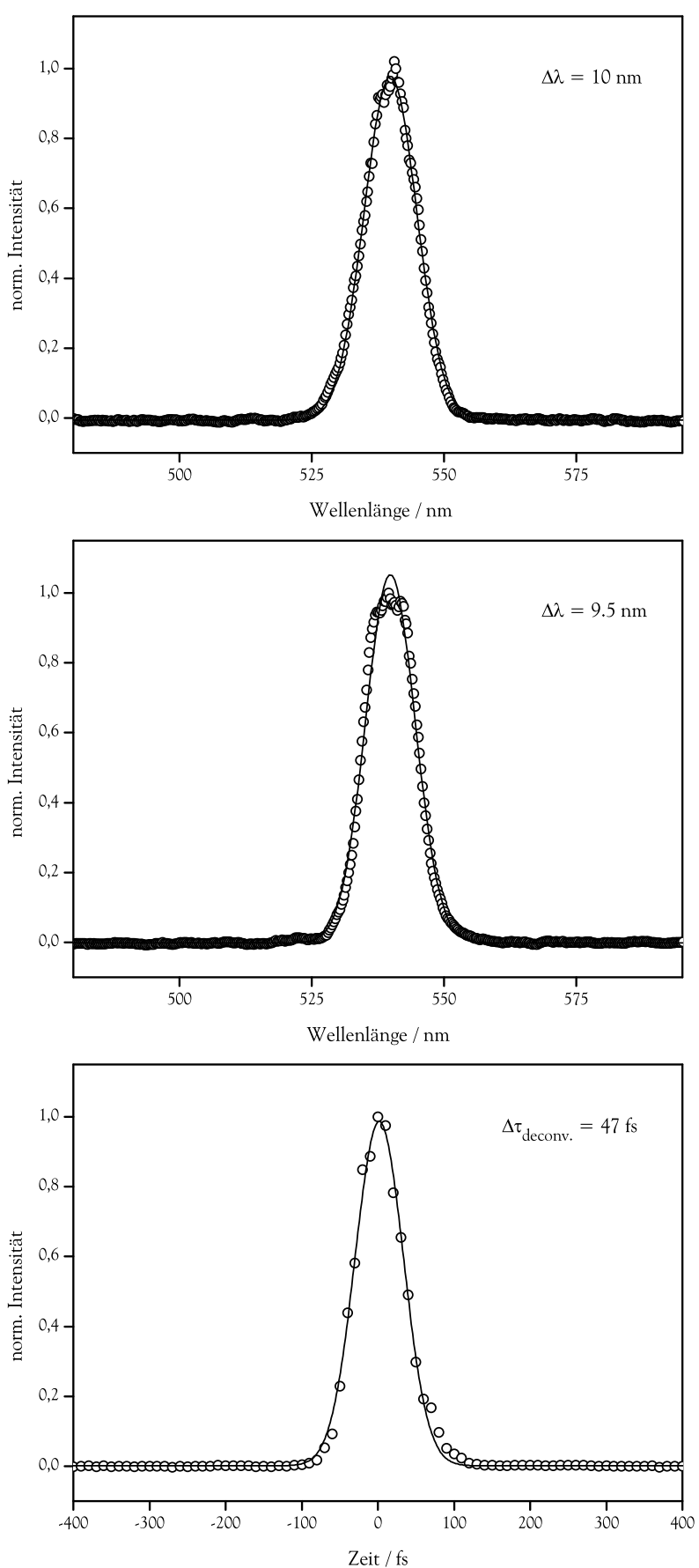

Abbildung 3.9: Spektren (oben: 1. Verstärkungsstufe, Mitte: 2. Verstärkungsstufe) und Autokorrelation (unten) des NOPA-Systems bei $540 \mathrm{~nm}$ 
den Pumpstrahl vom Signal ab. Zur Verstärkung des Signals werden der von ST2 transmittierte Pumpstrahl über M3, M4, M5 und HS2 $(r=200 \mathrm{~mm})$ sowie der Signalstrahl über M7, M8 und L3 im Kristall BBO3 (5x5x2 mm³, Typ I Phasenanpassung $\left.\left(32,5^{\circ}\right)\right)$ überlappt. Der Fokus beider Strahlen liegt auch in der zweiten Stufe einige Millimeter vor dem Kristall. Der Abstand des Kristalls vom Pumpfokus ist so gewählt, daß im beleuchteten Labor ein deutlicher Superfluoreszenzring hinter dem Kristall zu sehen ist. Die Anpassung der zeitlichen Verzögerung erfolgt über Translation von M5. Stimmen sowohl der Winkel $\theta$ zwischen den Strahlen als auch die Orientierung des Kristalls für optimale Phasenanpassung, erfolgt eine Verstärkung des Signals um einen Faktor $10^{3}$.

Mittels einer Glasfaser können die Pulse sowohl der ersten als auch der zweiten Stufe in ein Spektrometer (AvaSpec2048-USB, Avantes) eingekoppelt werden. Während der täglichen Justierung wurden erste und zweite NOPA-Stufe auf identische Zentralwellenlängen und gaußförmige spektrale Profile optimiert. In Abb. 3.9 sind beispielhaft die Spektren für $540 \mathrm{~nm}$, was der für die Messungen am Benzol benutzten Probewellenlänge von $270 \mathrm{~nm}$ entspricht, gegenübergestellt. Die Breite auf halber Höhe des Maximums beträgt $10 \mathrm{~nm}$ in der ersten und $9.5 \mathrm{~nm}$ in der zweiten Stufe (oberer bzw. mittlerer Graph). Im unteren Teil der Abbildung ist die Autokorrelation nach der Kompression der zweiten Stufe (s. S. 51) gezeigt, wobei die Länge der Einzelpulse durch Änderung der Kompressorparameter (Abstand der Prismen, Weglänge im Glas) auf 47 fs minimiert wurde. Die endgültig verstärkten Pulse können mit $\Delta \nu \cdot \Delta \tau=0.48$ als nahezu bandbreitelimitiert bezeichnet werden. Es ist jedoch zu berücksichtigen, daß die Pulse aufgrund der Gruppengeschwindigkeitsdispersion nach Durchlaufen der dispersiven Elemente im folgenden optischen Aufbau (Pump-Probe-Spektrometer, Meßzelle) zeitlich wieder etwas gestreckt werden (s. Zeitauflösung, S. 52).

\subsubsection{Das TOPAS-System: Erzeugung von IR-Pulsen}

Die im nahen Infrarotbereich von 1500 bis 2500 nm durchstimmbaren Pumppulse werden im Travelling Wave Optical Parametric Amlifier of Superfluorescence (TOPAS, Light Conversion Ltd.) erzeugt. Eine sehr detaillierte Beschreibung der damit verbundenen nichtlinearen optischen Prozesse sowie von Aufbau und Funktionsweise des Gerätes ist in [44] zu finden, daher soll hierauf im folgenden nur kurz eingegangen werden. 


\section{Prinzipielle Funktionsweise}

Des Prinzips der Superfluoreszenz zur Generierung von ultrakurzen IR-Pulsen bedienen sich generell zwei Arten von optisch parametrischen Verstärkern. In einem optisch parametrischen Oszillator (OPO) befindet sich das nichtlineare Medium in einem Resonator, welcher für Signal- und/oder Idlerwellenlänge optimiert ist. Hier ist ein einziger Durchgang durch das nichtlineare Medium nicht ausreichend, um eine genügend hohe Superfluoreszenzleistung zu erreichen. Der Resonator ermöglicht daher mehrere Durchgänge von Signal- und/oder Idlerpulsen durch das Medium, wobei jedesmal simultan ein weiterer Pumppuls den Kristall durchläuft, bis eine ausreichende Verstärkung erzielt ist und die Superfluoreszenz ausgekoppelt werden kann. Mit einem einzigen Pumppuls sind dagegen nur Energien unter $10^{-2} \mu \mathrm{J}$ realisierbar $[54,55]$.

Liegt nun die Pumpleistung pro Fläche im $\mathrm{GW} / \mathrm{cm}^{2}$-Bereich, reicht ein einziger Durchgang durch den Kristall, um eine der Pumpleistung entsprechende Superfluoreszenzleistung zu erhalten. Ein solcher Travelling-wave optisch parametrischer Generator (TOPG) benötigt daher keinen Resonator und ist somit besonders für den Einzelpulsbetrieb geeignet. Analog zum OPO kann allerdings die Kohärenz und Stabilität der generierten Pulse durch vermehrte Verstärkungsschritte erhöht werden. Nach diesem Prinzip funktioniert der in diesem Experiment verwendete Verstärker, bei dem in fünf Durchläufen durch einen einzelnen BBO-Kristall IR-Pulse mit Femtosekundenauflösung generiert und verstärkt werden.

Das TOPAS-System wird mit $80 \%$ der aus dem Hurricane ${ }^{\mathrm{TM}}$ ausgekoppelten Ti:Sa-Fundamentalen bei $800 \mathrm{~nm}$ gepumpt. Die für die Änderung der Signal/Idlerwellenlänge erforderliche Anpassung des Kristallswinkels und anderer optischer Komponenten (zeitliche Verzögerung zwischen Seed und Pump, Gitterwinkel) erfolgt computergesteuert anhand gespeicherter Eichkurven der Schrittmotorpositionen. Eine Justierung der optischen Komponenten innerhalb des TOPASAufbaus ist nicht vonnöten, lediglich die Einkoppelspiegel müssen optimiert werden. Desweiteren hängt die Leistung des TOPAS empfindlich von Pumppulslänge und -spektrum ab, die durch Anpassen der Hurricane-Kompressorlänge verbessert werden können. Bei einer Eingangsleistung von $800 \mathrm{~mW}$ (1 kHz Repetitionsrate) werden bei ausgewählten Wellenlängen Gesamtenergien (Signal, Idler, VIS) von $220 \mu \mathrm{J}$ erreicht, die einer Konversionseffizienz von max. $1.5 \%$ im Idler entsprechen. 

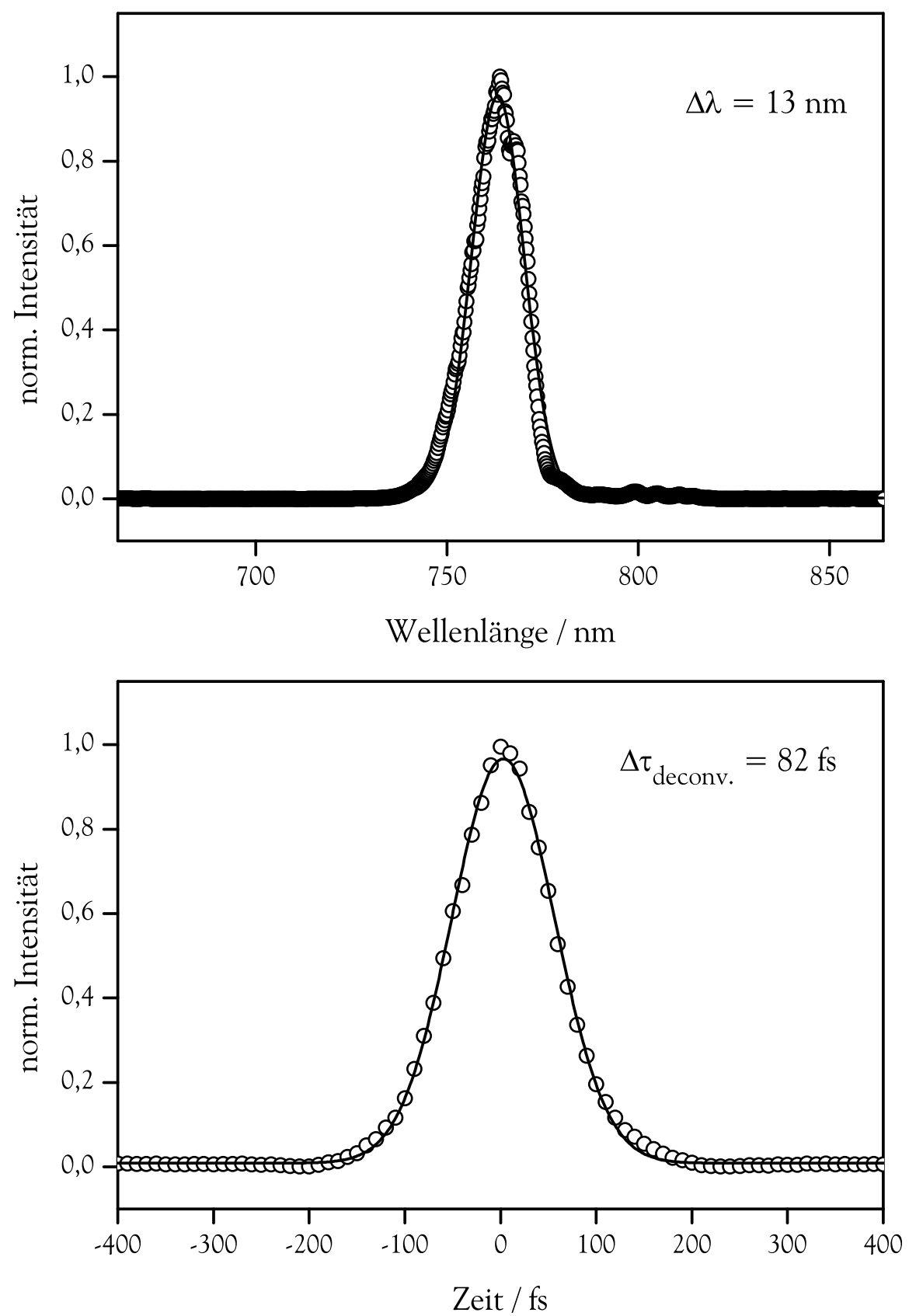

Abbildung 3.10: Spektrum (oben) und Autokorrelation (unten) des TOPASSystems, Zweite Harmonische von $\lambda_{\text {Idler }}=1670 \mathrm{~nm}$ 
Sowohl Signal- als auch Idlerpuls können mittels eines optional am Ausgang des TOPAS zu montierenden BBO-Kristalls (Typ I Phasenanpassung) frequenzverdoppelt werden, wodurch auch der VIS-Spektralbereich zugänglich wird. Diese Option wurde zum einen genutzt, um die Synchronisation des IR-Choppers (Ch in Abb. 3.11) anhand des Stroboskopeffektes zu optimieren. Zum anderen lassen die Charakteristika (Zentralwellenlänge, Spektrum, Autokorrelation) der frequenzverdoppelten Strahlung in diesem Fall direkt auf die Güte der zugrundeliegenden infraroten Pulse rückschließen. Laut Spezifikationen des Herstellers generiert das TOPAS-System über den gesamten zugänglichen Frequenzbereich nahezu bandbreite- und beugungsbegrenzte Pulse. Allerdings konnte dies in der Praxis nur bei ausgewählten Wellenlängen realisiert werden. Im Bereich typischer Anregungswellenlängen des ersten Obertons von aromatischen $\mathrm{CH}-$ Streckschwingungen $(\lambda \approx 1670 \mathrm{~nm})$ betrug $\Delta \tau \cdot \Delta \nu=0.57$. Dieser Wert wurde für die entsprechenden frequenzverdoppelten Idlerpulse mit einer Wellenlänge von $764 \mathrm{~nm}$ gemessen, wobei davon ausgegangen werden kann, daß die Fundamentalwelle sehr ähnliche Charakteristiken aufweist. Das zugehörige Spektrum und die Autokorrelation sind in Abb. 3.10 abgebildet.

\subsubsection{Das Pump-Probe-Spektrometer}

Die Experimente dieser Arbeit beruhen auf dem Prinzip der Pump-ProbeSpektroskopie. Durch Anregung mit einem Pump-Puls geeigneter Wellenlänge wird das System in einen Nichtgleichgewichtszustand gebracht, dessen Relaxation mit einem zeitverzögerten Probe-Puls beobachtet wird. Für die Untersuchungen zur Schwingungsrelaxation wurden ein Pumppuls mit Wellenlängen im nahen Infrarotbereich und ein Probepuls mit ultraviolettem Spektrum verwendet, was im Meßprinzip (vgl. Abschn. 3.1) begründet ist. Die Erzeugung der beiden Pulse erfolgt in den parametrischen Verstärkern, die in den Abschnitten 3.2.2 und 3.2.3 beschrieben wurden. Dieser Abschnitt beschäftigt sich nun mit dem Aufbau des eigentlichen Pump-Probe-Spektrometers. Die beiden Strahlengänge von Pumpund Probepuls sowie die elektronische Synchronisation und Datenaufnahme werden getrennt behandelt. 


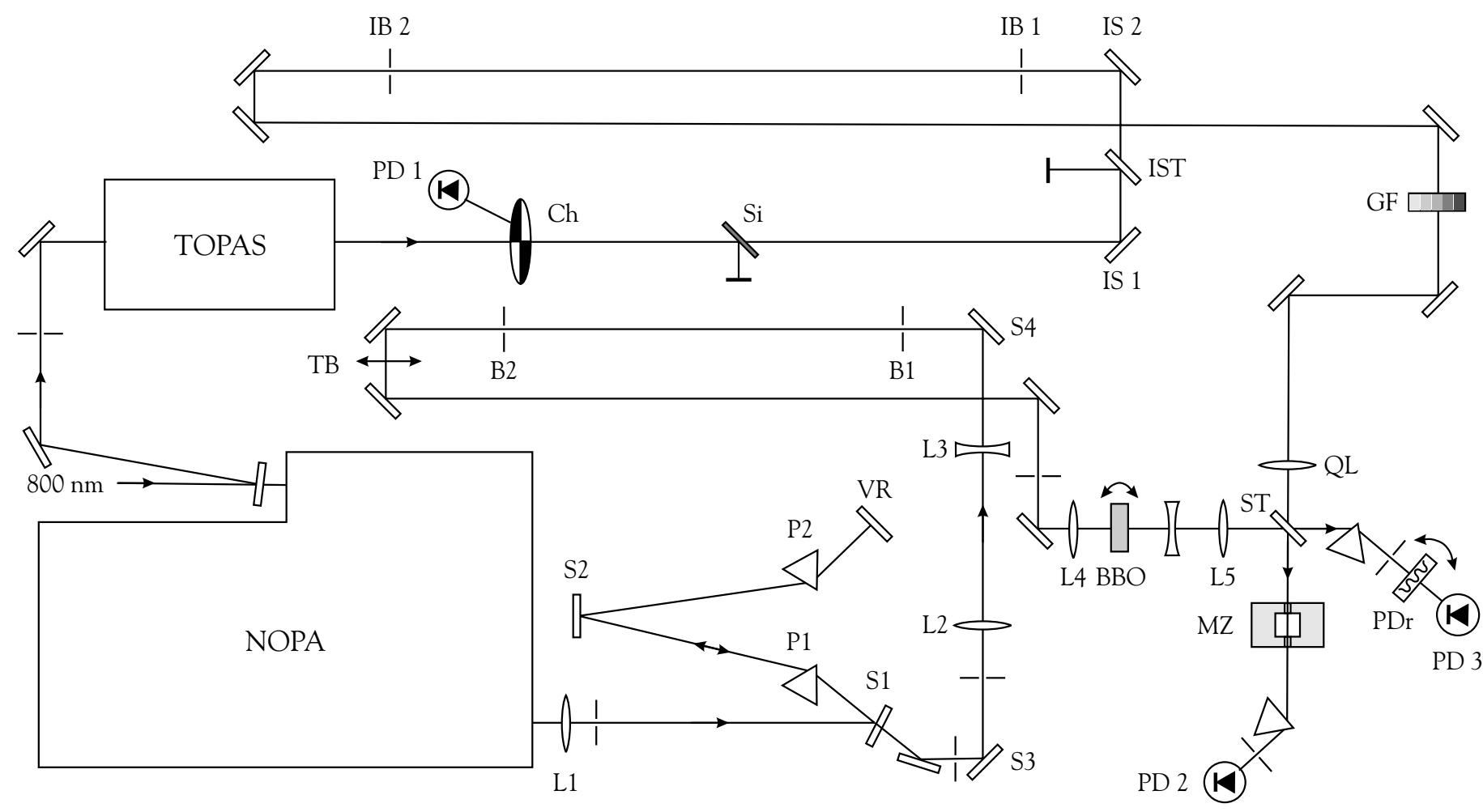

Abbildung 3.11: Optischer Aufbau des Pump-Probe-Spektrometers (Erläuterung der Abkürzungen s. Text) 
Kapitel 3 Experimentelles

\section{Der optische Aufbau}

Der Strahlengang des Pump-Probe-Spektrometers ist in Abb. 3.11 skizziert. Der IR-Pumppuls trifft nach Austreten aus dem TOPAS auf einen synchronisierten Chopper (Ch), der jeden zweiten Puls blockt und somit die Pulsrate auf $500 \mathrm{~Hz}$ halbiert. Die Reflektion des Lichtes am Chopper wird mit einer Photodiode PD1 registriert. Dies dient zur Reduktion des Rauschens bei der Signalaufnahme und wird im entsprechenden Abschnitt 3.2.5 genauer erläutert.

Da der Signal- und Idlerwelle noch Reste des sichtbaren Lichtes (Ti:SaFundamentale, Superfluoreszenz aus dem TOPAS) überlagert sind, wurde ein Siliziumplättchen $(\mathrm{Si})$ von $1 \mathrm{~mm}$ Dicke unter einem Winkel von $45^{\circ}$ in den Strahlengang gestellt, welches alle sichtbaren Anteile reflektiert. Da die Wellenlängen 1600 bis $2500 \mathrm{~nm}$, welche für die Experimente dieser Arbeit verwendet wurden, dem spektralen Bereich des Idlerpulses entsprechen, wird die Signalwelle am Infrarot-Strahlteiler (IST) abgetrennt und verworfen. Durch Justierung mittels der infrarotgeeigneten Goldspiegel IS1 und IS2 auf die Blenden IB1 bzw. IB2 wird der Strahlengang parallel zur Tischoberfläche auf einer den NOPA-Pulsen entsprechenden Höhe von $13 \mathrm{~cm}$ definiert. Für Wellenlängen um $1700 \mathrm{~nm}$ reicht eine IR-Karte zur Bestimmung der Strahlposition aus, im Bereich > $2000 \mathrm{~nm}$ wird ein IR-Sichtgerät zur Hilfe genommen bzw. der Strahl mittels einer Linse auf die IR-Karte fokussiert. Über einen horizontalen Retroreflektor und einige Spiegel, die sowohl zur Kompensation des Weglängenunterschiedes zwischen Pump- und Probestrahl als auch einem kompakten Aufbau dienen, wird der Strahl in die Meßanordnung dirigiert. Ein fünfstufiger Graufilter (GF) dient zur Anpassung der Pumpintensität, um z. B. die Bildung eines Kontinuums in den Fenstern der Meßzelle zu vermeiden. Mittels einer Quarzlinse (QL, $f=100$ oder $70 \mathrm{~mm}$ ) wird der Strahl schließlich in die Meßzelle fokussiert. Transmittiertes IR-Licht wird hinter der Zelle mit einem Strahlblocker aufgefangen.

Die NOPA-Pulse sind aufgrund der dispersiven Elemente im Verstärker zeitlich verlängert worden. Nach Parallelisierung mittels der Linse L1 und Abtrennen von Pumpstrahl und Superfluoreszenz an einer Blende wird das sichtbare Licht daher in einen Prismenkompressor mit gefalteter Geometrie geleitet. Der Spiegel S1 reflektiert das $12 \mathrm{~cm}$ über der Tischoberfläche laufende Licht auf die Spitze des Prismas P1, an dem es in Richtung des Spiegels S2 gebrochen wird. Nach Beugung an einem zweiten Prisma P2 trifft der Strahl auf den vertikalen Retroreflektor VR 
und wird um $1 \mathrm{~cm}$ nach oben versetzt. Anschließend durchläuft der Strahl den Aufbau in umgekehrter Reihenfolge und wird oberhalb von S1 ausgekoppelt.

Ein Teleskop (L2 und L3) reduziert den Strahldurchmesser auf $2 \mathrm{~mm}$. Eine Anpassung des Strahlengangs mittels der Spiegel S3 und S4 unter Zuhilfenahme der Blenden B1 und B2 stellt sicher, daß der Strahl in $13 \mathrm{~cm}$ Höhe und parallel zur Bewegegungsrichtung der linearen Translationsbühne TB (Schrittmotor UE63PP, Steuereinheit MM4000, Newport, Auflösung $1 \mu \mathrm{m}$ ) verläuft. Auf der Verschiebebühne ist ein horizontaler Retroreflektor montiert. Eine Translation der Bühne um $0.3 \mathrm{~mm}$ resultiert wegen dieser gefalteten Geometrie in einer zeitlichen Verzögerung von 2 ps. Anschließend wird der Puls mittels zweier Spiegel zur Linse L4 gelenkt, welche ihn in den BBO-Kristall BBO $\left(7 \times 7 \times 0.2 \mathrm{~mm}^{3}\right.$, Typ I Phasenanpassung $\left(32.5^{\circ}\right)$ ) fokussiert. Hier erfolgt Frequenzverdopplung des sichtbaren Lichtes zur Erzeugung des UV-Probepulses. Dieser wird anschließend mittels der Linse L5 fokussiert und am Strahlteiler ST im Verhältnis 1:1 in Meßs- und Referenzstrahl geteilt. Die Reflektion des Probepulses wird mit dem Pumppuls in der Meßzelle (MZ) überlappt, im Falle der Gasphasenmessungen kollinear, andernfalls unter einem Winkel von 5-15. Sowohl der Meß- als auch der Referenzstrahl werden mit Prismen spektral zerlegt, da beide noch Reste sichtbaren Lichtes aufgrund der unvollständigen Konversion in BBO3 enthalten. Mit Blenden werden jeweils die ultravioletten Anteile selektiert, die dann von Photodioden (S126-8BQ, Hamamatsu) als $I$ (PD2) und $I_{0}$ (PD3) integriert registriert werden. Ein Polarisationsdreher (PDr) vor der $I_{0}$-Diode ermöglicht es, die Signalintensitäten der beiden Dioden durch Abschwächen der Referenzintensität anzugleichen.

\section{Elektronische Synchronisation}

Die elektronischen Komponenten der Signalaufnahme und -verarbeitung müssen exakt miteinander synchronisiert sein. Die Laserpulse verlassen das HurricaneSystem mit einer Repetitionsrate von $1 \mathrm{kHz}$. Ein hierauf abgestimmter Trigger gleicher Frequenz dient als Eingangsignal der elektronischen Verzögerungseinheit (DG 535, Stanford Systems), die die folgenden Komponenten steuert: das DiodenNetzteil, die Chopper-Steuerung, sowie die Analog-Digital-Messkarte (DT3001, Data Translation). Das Dioden-Netzteil erhält ohne Verzögerung einen TriggerTTL-Impuls und aktiviert für $400 \mu$ s die Signal-, Referenz- und Chopperdioden. Um die Drehung der Chopperscheibe auf die TOPAS-Pulse abzustimmen, wird die Chopper-Steuerung ebenfalls mittels des DG535 mit dem Trigger des Hurricane 
Kapitel 3 Experimentelles

synchronisiert. Die Aktivierung der Meßkarte erfolgt $100 \mu$ s nach dem HurricaneTrigger. Innerhalb eines Zeitfensters von $100 \mu$ s liest sie die integrierten Signale der drei Dioden aus. Ein simultan mit der Meßkarte getriggertes Oszilloskop (LeCroy9400A) erlaubt es, die Meßwerte der Signal- und Referenzdiode zu kontrollieren und abzugleichen.

\section{Zeitauflösung}

Die Zeitauflösung des Experiments ist durch die Breite der Kreuzkorrelation zwischen Anregungs- und Abfragepuls begrenzt. Der obere Graph in Abb. 3.12 zeigt die Kreuzkorrelation des IR-Pumppulses bei $1670 \mathrm{~nm}$ und des UV-Probepulses bei $270 \mathrm{~nm}$, die in einem $1 \mathrm{~mm}$ dicken Saphirfenster überlappt wurden. Das temporale Profil kann als annähernd gaussförmig beschrieben werden und besitzt eine Halbwertsbreite von $\Delta \tau=570 \mathrm{fs}$. Damit fällt die Korrelation deutlich breiter aus, als eine Faltung zweier Pulse mit $\Delta \tau \approx 100$ fs erwarten ließe. Diese Diskrepanz ist auf den großen Unterschied der Gruppengeschwindigkeiten von Pump- und Probepuls in optisch dichten Medien $(n>1)$ zurückzuführen. Dementsprechend schmaler ist die Kreuzkorrelation, die im unteren Graphen von Abb. 3.12 abgebildet ist. Sie wurde in einem lediglich $0.2 \mathrm{~mm}$ dicken Saphirfenster aufgenommen, wie es für die Messungen in flüssiger Lösung in $[13,43,44]$ Verwendung fand. Hier beträgt die Halbwertsbreite ca. 140 fs. Es ist zu erwarten, daß die Kreuzkorrelation von Pump- und Probepuls bei kollinearem Strahlengang in der Gasphasenzelle mit $2 \mathrm{~cm}$ optischer Weglänge und $1 \mathrm{~mm}$ dicken Saphirfenstern noch stärker verlängert ist. Hier mußte ein Kompromiß zwischen gerade noch detektierbarer Signalintensität (siehe Abschn. 3.2.5) und optimaler Zeitauflösung eingegangen werden.

\subsubsection{Signalaufnahme und Signal-zu-Rausch-Verhältnis}

Die Observable in diesem Experiment ist die zeitliche Änderung der Absorbanz $A$, auch optische Dichte OD genannt. Sie ist wie folgt definiert

$$
A=\mathrm{OD}=-\lg \left(\frac{I}{I_{0}}\right) .
$$



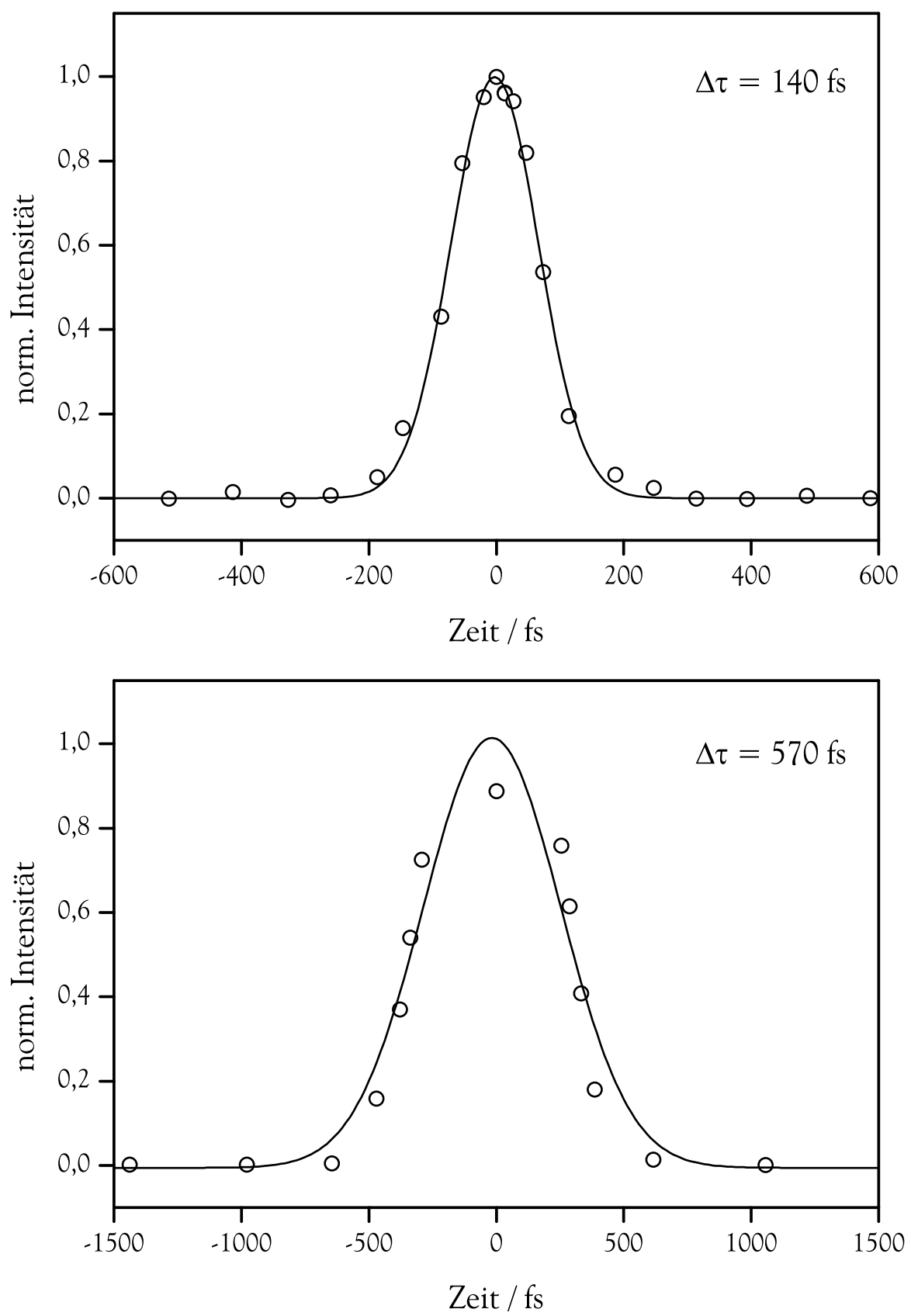

Abbildung 3.12: Kreuzkorrelation von Pump- und Probepuls in $0.2 \mathrm{~mm}$ Saphir (oben) und $1 \mathrm{~mm}$ Saphir (unten) 
Kapitel 3 Experimentelles

Dabei sind dem Lambert-Beer'schen Gesetz entsprechend $I_{0}$ die in ein Medium einfallende Lichtintensität (Referenzstrahl) und $I$ die Intensität des vom Medium transmittierten Lichts. Die zeitliche Abhängigkeit der optischen Dichte wird durch

$$
\mathrm{OD}(t)=-\lg \left(\frac{I(t)}{I_{0}(t)}\right)
$$

beschrieben. Mit der in Abschnitt 3.2.4 beschriebenen Chopper-Anordnung wird abwechselnd die durch die Anregung induzierte Absorption (Situation „Chopper AUF") und die thermische Absorption bei der Meßtemperatur $T_{\text {meß }}$ ohne Anregung („,Chopper ZU“) sowohl für das Probe- als auch das Referenzsignal registriert

$$
\begin{aligned}
\mathrm{OD}_{\mathrm{AUF}} & =\mathrm{OD}(t) \\
\mathrm{OD}_{\mathrm{ZU}} & =\mathrm{OD}_{T_{\mathrm{meB}}} .
\end{aligned}
$$

Auf diese Weise wird die zeitliche Änderung der Intensitäten im Vergleich zum Meßtemperaturwert gemessen. Dazu wird die Differenz

$$
\begin{aligned}
\Delta \mathrm{OD} & =\mathrm{OD}_{\mathrm{AUF}}-\mathrm{OD}_{\mathrm{ZU}} \\
& =-\lg \left(\frac{I_{\mathrm{AUF}}}{I_{0, \mathrm{AUF}}}\right)+\lg \left(\frac{I_{\mathrm{ZU}}}{I_{0, \mathrm{ZU}}}\right) \\
& =-\lg \left(\frac{I(t)}{I(t)_{0}}\right)+\lg \left(\frac{I_{T_{\text {meB }}}}{I_{0, T_{\text {meB }}}}\right)
\end{aligned}
$$

berechnet.

Der Vorteil dieser Methode besteht darin, daß durch kurze Laserschwankungen hervorgerufene Signalstörungen minimiert werden. Trotz der halbierten Anregungsrate wird auf diese Weise eine erhebliche Verbesserung des Signal-zuRausch-Verhältnisses errreicht.

Die Intensitäten der Gasphasensignale betrugen aufgrund der sehr niedrigen Substratkonzentrationen selten mehr als $100 \mu \Delta$ OD. Mit einem durchschnittlichen Rauschen von $\pm 100-150 \mu \Delta$ OD bei 1000 gemittelten Pump-ProbePulspaaren pro Meßpunkt wurden meistens acht Messungen hintereinander durchgeführt und zu einer transienten Spur gemittelt. Eine längere Meßdauer war nicht ratsam, da das Lasersystem sich geringfügig dejustieren und somit den kollinearen Überlapp von Pump- und Probestrahl beeinträchtigen konnte. Die im Kapitel 6 gezeigten Absorptions-Zeit-Profile sind Ergebnisse von zwischen 20 und 40 miteinander gemittelten Spuren. Das Signal-zu-Rausch-Verhältnis konnte auf diese Weise auf über fünfzehn verbessert werden. 


\subsection{Realisierung weiter Druck- und Temperaturbereiche}

Die Zielsetzung, den Einfluß der molekularen Umgebung auf die Schwingungsenergieumverteilung zu erforschen, verlangt nach vergleichenden Experimenten in der Gasphase, der überkritischen Phase und in Lösung. Je nach Lösungsmittel müssen somit weite Temperatur- und Druckbereiche zugänglich gemacht werden, die jeweils einen eigenen apparativen Ansatz erfordern. Für die Messungen bei erhöhten Temperaturen in der Gasphase und in überkritischen Medien stand eine kombinierte Hochdruck-/Vakuumapparatur zur Verfügung, an welche je nach gewünschten Bedingungen zwei verschiedene Meßzellen angeschlossen werden konnten.

\subsubsection{Die Hochdruck-/Vakuumapparatur}

Für diese Arbeit wurden Experimente über einen Druckbereich von 0.5 bis zu einigen hundert Bar durchgeführt. $\mathrm{Zu}$ diesem Zweck wurde eine Kombination aus Hochdruckstand und Vakuumapparatur konzipiert. So war es möglich, ohne Umbauten in einer Art von Meßzelle beliebige Druckbedingungen zu schaffen. Abb. 3.13 zeigt den schematischen Aufbau der Druckapparatur. Im folgenden werden die einzelnen Komponenten in der Reihenfolge beschrieben, in der sie für eine typische Messung eingesetzt werden.

Die Meßzelle (Ze) kann über den Dreiwegehahn V6 und das Ventil V5 mittels einer Vakuumpumpe VK evakuiert werden. Dabei sind alle anderen Ventile geschlossen. Die Verbindung zwischen V5 und der Pumpe wird über einen Teflonschlauch mit $6 \mathrm{~mm}$ Innendurchmesser hergestellt, welcher nur Drücken kleiner als 5 bar ausgesetzt werden darf. Sollte in der Apparatur ein höherer Druck anliegen, muß dieser über das Ventil V4 gemindert werden. Der Druck in der Zelle wird mittels eines Druckaufnehmers DM2 (HTT-25A, Keller) gemessen, der für einen Druckbereich von 0 bis 20 bar ausgelegt ist. Sollen in der Zelle höhere Drücke als 20 bar realisiert werden, wird DM2 durch einen Blindstopfen ersetzt.

Die zu messende Substanz wird in ein Substanzreservoir SR eingefüllt und kann bei Bedarf in mehreren Einfrier-Auftau-Zyklen über V5 und V6 entgast werden. Anschließend wird V5 geschlossen und über V6 das Substanzreservoir mit der Zelle verbunden. Ist durch Erwärmen des Reservoirs in der Zelle der gewünschte 


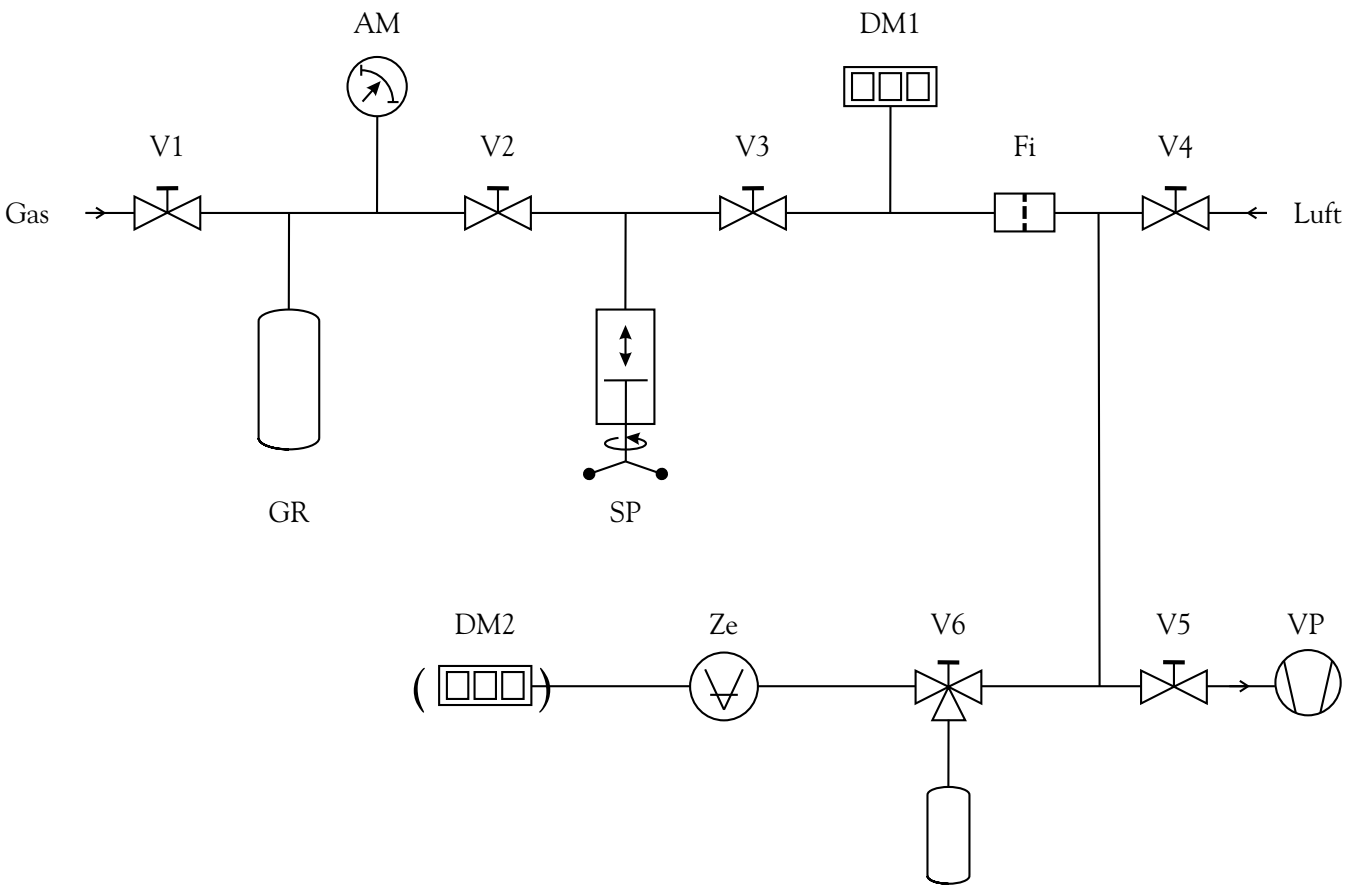

SR

Abbildung 3.13: Schematischer Aufbau der Hochdruck-/Vakuumapparatur (Erklärung der Abkürzungen siehe Text)

Substanzdruck eingestellt, wird es mittels V6 wieder verschlossen. Das Reservoir ist für Drücke bis zu 5 bar konzipiert.

Für die Messungen mit einem inerten Stoßpartner wird der Hochdruckteil der Apparatur verwendet. Bis auf die Verbindung zwischen V5 und der Pumpe sowie das Substanzreservoir sind sämtliche Leitungen für Drücke bis 1000 bar ausgelegt. Im Bereich von V1 bis V3 werden Hochdruckrohre mit 1/16" Innendurchmesser und 1/4" Außendurchmesser verwendet. Von V3 bis zur Zelle sind Hochdruckkapillaren mit $0.25 \mathrm{~mm}$ Innen- und 1/16 Zoll Außendurchmesser eingesetzt worden. Dies dient zum einen dazu, das Volumen der Gasleitung gering zu halten, zum anderen erlaubt es die Flexibilität der Kapillaren, die Zelle zu bewegen.

Für die Hochdruckbefüllung bleiben V4, V5 und V6 zunächst geschlossen. Eine beliebige Menge Gas wird über das Ventil V1 in das Gasreservoir GR geleitet und bei Gasen mit geeignetem Siedepunkt eventuell einkondensiert. Der Druck 
im Gasreservoir wird anhand des Zeigermanometers ZM (Anzeige 0 bis 250 bar) kontrolliert. Bei geschlossenem V3 wird über V2 der Kolben der Spindelpresse SP (NOVA-Swiss) in umkomprimiertem Zustand befüllt. Um einen höheren Vordruck zu erreichen, kann das Gasreservoir vorsichtig erwärmt werden. Bei geschlossenem V2 und offenen V3 erfolgt dann eine Kompression des Gases durch Drehen der Pressenspindel. Der Druck im Kapillarsystem wird über das digitale Manometer DM1 (Serie 10, Keller, Anzeige 0 bis 999 bar) gemessen. Ein HPLC-Filter (Fi) verbindet den Hochdruckstand mit dem Kapillarsystem. Über V6 wird das komprimierte Gas schließlich in die Zelle geleitet. Gegebenenfalls muß die Prozedur des Komprimierens mehrmals wiederholt werden, wenn sehr hohe Enddrücke in der Zelle erreicht werden sollen.

\subsubsection{Die Meßzellen}

Ein großer Vorteil der IR-Pump-UV-Probe-Spektroskopie liegt in ihrer Vielseitigkeit. Durch den Einsatz verschiedener Meßzellen mit variabler Schichtdicke und großzügig wählbaren Temperatur- und Druckbedingungen konnte im Rahmen dieser Arbeit ein- und dieselbe Substanz in verschiedenen Aggregatzuständen untersucht werden.

\section{Die Gasphasen-Zelle}

Für die Experimente in der Gasphase wurde eine neue kompakte Zelle konzipiert und angefertigt. Sie besteht aus einem aus einem Stück gefertigten Stahlkörper, der mit einem passenden Flansch abgedichtet wird. Das eingeschlossene Gasvolumen beträgt ca. $20 \mathrm{ml}$ bei einer Schichtdicke zwischen 20 und $23 \mathrm{~mm}$, abhängig von der Dicke der eingesetzten Saphirfenster. Im Falle der Messungen an reinen gasförmigen Substanzen und Gasmischungen bis zu 30 bar wurde direkt an das Probevolumen ein Drucktransmitter (HTT-25A, Keller) angeflanscht, der für die erforderlichen Betriebstemperaturen von bis zu $160^{\circ} \mathrm{C}$ geeignet ist. Waren höhere Drücke in der Zelle erforderlich, wurde der Transmitter durch einen Blindstopfen ersetzt. Sowohl die Zelle als auch der direkt daran angeschlossene Doppelhahn wurden mit vier bzw. zwei Heizpatronen (50-250 V bzw. $40 \mathrm{~V}$, HotSet) und je einem Pt100-Temperatursensor bestückt und über separate PID-Feedback-Regler (Micromega CN77000, Omega) auf die gewünschte Meßstemperatur eingestellt. Durch die Temperierung des Hahns wurde sichergestellt, daß die Substanz nicht 
Kapitel 3 Experimentelles

zwischen Substanzreservoir und Meßvolumen kondensierte. Die Schwankungen der Zelltemperatur betrugen maximal $\pm 1^{\circ} \mathrm{C}$.

\section{Die Zelle für überkritische Lösungen}

Diese Stahlzelle besteht aus zwei gleich dicken Hälften, die aneinandergesetzt ein Volumen von ca. $1 \mathrm{ml}$ einschließen. Überkritische Lösungen neigen in der Nähe des kritischen Punktes leicht zu Schlierenbildung, wodurch Pump- und Probestrahl unregelmäßig abgelenkt werden und die Detektion einer Absorptionsänderung erheblich erschwert wird. Durch Integration eines Magnetrührers in die Zelle für überkritische Medien konnte dieses Problem behoben werden. Die hohe Dichte des Fluids führt darüber hinaus zu einem ausgeprägten Unterschied der Gruppengeschwindigkeiten von Pump- und Probepulsen, so daß die Schichtdicke der Meßzelle limitiert werden muß. In der vorliegenden Konstruktion sind optische Weglängen von 4-5 mm, je nach Wahl der eingesetzten Saphirfenster, möglich. Auch an diesen Zellkörper läßt sich der oben beschriebene Drucktransmitter bis 30 bar anschließen. Für Drücke bis 750 bar wird der Transmitter wieder durch einen Blindstopfen ersetzt und der Druck im Kapillarsystem (DM1 in Abb. 3.13) gemessen. Bei Bedarf wurde zwischen Hochdruckstand und Zelle der beheizbare Doppelhahn mit separatem Substanzreservoir eingefügt. Substanzen mit geringem Dampfdruck wurden z. T. auch direkt in die Zelle eingefüllt. Anhand vierer über eine PID-Regelung gesteuerter Heizpatronen konnte die Fluidtemperatur auf bis zu $200^{\circ} \mathrm{C}$ mit einer Genauigkeit von $\pm 1{ }^{\circ} \mathrm{C}$ eingestellt werden. 


\section{Kapitel 4}

\section{Modellsysteme}

Die im Rahmen dieser Arbeit angewandte Technik der IR-Pump-UV-ProbeSpektroskopie bietet u. a. die Möglichkeit, durch vergleichende Experimente an chemisch sehr ähnlichen Modellsystemen den Effekt chemischer Substitution auf den Schwingungsenergietransfer zu untersuchen. Dabei sind bei der Wahl der Modellsysteme nur wenige Bedingungen zu beachten, die im folgenden kurz aufgeführt sind.

- Die eindeutige Interpretation der Pump-Probe-Daten auf Grundlage des in Abschn. 3.1 vorgestellten Meßprinzips ist nur möglich, wenn der IR-Pumppuls einen hellen Zustand mit lokalisierter Anregungsenergie präpariert. Dies erfordert eine entsprechend isolierte Absorptionsbande im Infrarotspektrum des Moleküls. Im Fall des ersten Obertons der $\mathrm{CH}-$ Streckschwingungen bei ca. $6000 \mathrm{~cm}^{-1}$ sowie der Kombinationsbanden von CH-Streck- mit CC-Streck- und $\mathrm{CH}$-Biegeschwingungen bei ca. $4500 \mathrm{~cm}^{-1}$ ist diese Bedingung von allen Modellsystemen dieser Arbeit erfüllt.

- Um eine Mindestmenge an deponierter Anregungsenergie und somit eine Mindestintensität des Pump-Probe-Signals zu gewährleisten, sollte der molare Absorptionskoeffizient bei der jeweiligen Pumpwellenlänge mehr als $0.51 \mathrm{~mol}^{-1} \mathrm{~cm}^{-1}$ betragen .

- Der Abfragevorgang mittels eines ultravioletten fs-Laserpulses darf keine photochemische Reaktion wie z. B. eine Isomerisierung oder Photodimerisierung verursachen, die eine zweite absorbierende Spezies generieren würde. 
Kapitel 4 Modellsysteme

- Die Experimente an den reinen gasförmigen Modellsystemen konnten in einem Temperaturbereich bis $150^{\circ} \mathrm{C}$ durchgeführt werden. Um eine vertretbare Signalintensität zu erreichen, muß der Dampfdruck der Substanz bei dieser Temperatur mindestens 0.5 bar betragen.

- Die Kalibration der transienten Absorption über bei Temperaturen zwischen 50 und $500^{\circ} \mathrm{C}$ gemessene UV-Absorptionsspektren setzt voraus, daß das Modellsystem in diesem Temperaturbereich thermisch stabil ist.

Die fünf in dieser Arbeit untersuchten Modellsysteme gehören zur Klasse der Aromaten mit sechsgliedrigem Ring. Neben dem einfachsten Molekül dieser Klasse, Benzol $\left(\mathrm{C}_{6} \mathrm{H}_{6}\right)$, wurden verschiedene monosubstituierte Derivate studiert. Es handelt sich dabei um monodeuteriertes Benzol- $\mathrm{d}_{1}\left(\mathrm{C}_{6} \mathrm{H}_{5} \mathrm{D}\right)$, Chlorbenzol $\left(\mathrm{C}_{6} \mathrm{H}_{5} \mathrm{Cl}\right)$ sowie zwei Derivate mit polyatomarem Substituenten, Toluol $\left(\mathrm{C}_{6} \mathrm{H}_{5} \mathrm{CH}_{3}\right)$ und $\alpha, \alpha, \alpha$-Trifluortoluol $\left(\mathrm{C}_{6} \mathrm{H}_{5} \mathrm{CF}_{3}\right)$. Für Benzol-d $\mathrm{d}_{1}$ und Chlorbenzol stellen die Daten dieser Arbeit die ersten mit der IR-Pump-UV-ProbeSpektroskopie gewonnenen Ergebnisse dar. Die übrigen drei Modellsysteme wurden bereits zuvor mit dieser Technik untersucht, allerdings nicht in der Gasphase, sondern in flüssigen Lösungsmitteln [13,43] oder in überkritischem $\mathrm{CO}_{2}$ [43]. Unter Einbeziehung von [44] bildet diese Arbeit den Abschluß einer vergleichenden Studie des Energietransfers in aromatischen Modellsystemen in verschiedenen molekularen Umgebungen.

Dieses Kapitel widmet sich besonders den spektralen Eigenschaften der fünf Modellsysteme. Detaillierte Analysen der IR-Absorptionsspektren im Energiebereich der Anregungspulse können Aufschluß über die Natur des hellen Zustands nullter Ordnung (vgl. Abschn. 2.1) geben. Ein Vergleich der Normalmoden der Moleküle zeigt darüber hinaus ihre enge Verwandtschaft auf, die es rechtfertigt, die Substitution lediglich als Störung (im Gegensatz zu einer prinzipiellen Veränderung) der Benzolstruktur aufzufassen.

Die Franck-Condon-aktiven Normalmoden der Moleküle, welche die Absorption des Probepulses bedingen, können anhand von UV-Spektren und Fluoreszenzdaten benannt werden. Auch hier kann über eine symmetriebasierte Klassifikation der Normalschwingungen die vergleichende Interpretation der Pump-ProbeSignale gerechtfertigt werden.

Eine Absorbanz im langwelligen Bereich der UV-Spektren ist mit Übergängen aus thermisch schwingungsangeregten Zuständen der Modellsysteme verbunden. 
Ein Anstieg der Absorbanz, wie in den Pump-Probe-Zeitprofilen beobachtet, entspricht demnach einer Erhöhung der Temperatur. Die Temperaturabhängigkeit der Absorptionskoeffizienten bei einer Wellenlänge läßt sich z. B. in Stoßwellenmessungen ermitteln $[13,43,44,56]$. Im Rahmen dieser Arbeit war es erforderlich, mehrere Wellenlängen simultan in temperaturabhängigen Messungen zu erfassen, deswegen wurde hier auf heiße Absorptionsspektren bei Temperaturen bis $800 \mathrm{~K}$ zurückgegriffen. Die statistische Thermodynamik erlaubt eine Umrechnung der Temperatur in die Innere Energie des Moleküls, so daß die Energieabhängigkeit der Absorptionskoeffizienten einzelner Wellenlängen angegeben werden kann. Hierfür kann ausgenutzt werden, daß die Absorptionskoeffizienten eines kanonischen und eines mikrokanonischen Ensembles identisch sind, sofern die mittleren Energien der Verteilungen übereinstimmen [34,57]. Die einzelnen Kalibrationsmessungen sind am Ende jedes Modellsystemabschnitts gezeigt.

Dieses Kapitel schließt mit einem Vergleich der Modellsysteme in Hinblick auf ihre Zustandsdichten und QNS-Struktur. 


\subsection{Benzol}

Von den fünf in dieser Arbeit untersuchten aromatischen Molekülen stellt das Benzol $\mathrm{C}_{6} \mathrm{H}_{6}$ das Referenzsystem dar. Die Auswirkung einer Monosubstitution mit einem Deuterium- oder Chloratom bzw. einer Methylgruppe oder ihrem perfluorierten Analogon auf den IVR-Prozess zeigt sich erst im Vergleich mit dem unsubstituierten Muttermolekül.

Als einfachster Sechsringaromat besitzt Benzol im Grundzustand $\mathrm{D}_{6 \mathrm{~h}}$-Symmetrie. In dieser Punktgruppe faktorisieren die 30 Normalschwingungen des Moleküls in zwölf irreduzible Darstellungen mit der Gewichtung $2 \mathrm{a}_{1 \mathrm{~g}}+\mathrm{a}_{2 \mathrm{~g}}+2 \mathrm{~b}_{1 \mathrm{u}}+2 \mathrm{~b}_{2 \mathrm{u}}+6 \mathrm{e}_{1 \mathrm{u}}+8 \mathrm{e}_{2 \mathrm{~g}}$ und $1 \mathrm{a}_{2 \mathrm{u}}+2 \mathrm{~b}_{2 \mathrm{~g}}+2 \mathrm{e}_{1 \mathrm{~g}}+4 \mathrm{e}_{2 \mathrm{u}}$. Die Bewegungsvektoren der ersten Gruppe von Normalmoden liegen in der Molekülebene, die der zweiten Gruppe außerhalb davon. Diejenigen Schwingungen, die $e_{x x}$-Symmetrie aufweisen, treten zweifach entartet auf, weswegen nur zwanzig verschiedene Fundamentalfrequenzen für dieses Molekül benannt werden müssen. Eine Auflistung dieser Schwingungen samt ihrer Symmetrien ist in Tabelle $4.1 \mathrm{zu}$ finden. Entgegen der sonst gängigen Herzberg-Nomenklatur für Fundamentalschwingungen, bei der die Moden zunächst nach ihrer Symmetrie gruppiert und dann nach absteigender Energie geordnet werden, sind die

\begin{tabular}{|lcll|lcll|}
\hline Mode & $\begin{array}{c}\text { Energie } \\
{\left[\mathbf{c m}^{-\mathbf{1}}\right]}\end{array}$ & Sym. & Wnr. & Mode & $\begin{array}{c}\text { Energie } \\
{\left[\mathbf{c m}^{-1}\right]}\end{array}$ & Sym. & Wnr. \\
\hline \hline$\nu_{1}$ & 3073 & $\mathrm{a}_{1 \mathrm{~g}}$ & 2 & $\nu_{16,17}$ & 1038 & $\mathrm{e}_{1 \mathrm{u}}$ & $18(\mathrm{a}, \mathrm{b})$ \\
$\nu_{2,3}$ & 3064 & $\mathrm{e}_{1 \mathrm{u}}$ & $20(\mathrm{a}, \mathrm{b})$ & $\nu_{18}$ & 1010 & $\mathrm{~b}_{1 \mathrm{u}}$ & 12 \\
$\nu_{4}$ & 3057 & $\mathrm{~b}_{1 \mathrm{u}}$ & 13 & $\nu_{19}$ & 993 & $\mathrm{a}_{1 \mathrm{~g}}$ & 1 \\
$\nu_{5,6}$ & 3056 & $\mathrm{e}_{2 \mathrm{~g}}$ & $7(\mathrm{a}, \mathrm{b})$ & $\nu_{20}$ & 990 & $\mathrm{~b}_{2 \mathrm{~g}}$ & 5 \\
$\nu_{7,8}$ & 1600 & $\mathrm{e}_{2 \mathrm{~g}}$ & $8(\mathrm{a}, \mathrm{b})$ & $\nu_{21,22}$ & 967 & $\mathrm{e}_{2 \mathrm{u}}$ & $17(\mathrm{a}, \mathrm{b})$ \\
$\nu_{9,10}$ & 1483 & $\mathrm{e}_{1 \mathrm{u}}$ & $19(\mathrm{a}, \mathrm{b})$ & $\nu_{23,24}$ & 847 & $\mathrm{e}_{1 \mathrm{~g}}$ & $10(\mathrm{a}, \mathrm{b})$ \\
$\nu_{11}$ & 1350 & $\mathrm{a}_{2 \mathrm{~g}}$ & 3 & $\nu_{25}$ & 707 & $\mathrm{~b}_{2 \mathrm{~g}}$ & 4 \\
$\nu_{12}$ & 1309 & $\mathrm{~b}_{2 \mathrm{u}}$ & 14 & $\nu_{26}$ & 673 & $\mathrm{a}_{2 \mathrm{u}}$ & 11 \\
$\nu_{13,14}$ & 1177 & $\mathrm{e}_{2 \mathrm{~g}}$ & $9(\mathrm{a}, \mathrm{b})$ & $\nu_{27,28}$ & 608 & $\mathrm{e}_{2 \mathrm{~g}}$ & $6(\mathrm{a}, \mathrm{b})$ \\
$\nu_{15}$ & 1149 & $\mathrm{~b}_{2 \mathrm{u}}$ & 15 & $\nu_{29,30}$ & 398 & $\mathrm{e}_{2 \mathrm{u}}$ & $16(\mathrm{a}, \mathrm{b})$ \\
\hline
\end{tabular}

Tabelle 4.1: Die 30 Normalschwingungen des Benzols, nach absteigender Energie sortiert [58], Wnr.: Wilson-Nummer [59] 
Schwingungen in diesem und den folgenden Abschnitten nach absteigender Frequenz numeriert. Dies erleichtert den Vergleich mit den Benzolderivaten anderer Gesamtsymmetrie. Ein weiteres Vergleichskriterium bietet die von Wilson eingeführte Nomenklatur für Benzol [59], die sich weitesgehend auf die Phenylschwingungen der monosubstituierten Derivate übertragen läßt [60]. Die entarteten Normalmoden werden in Wilsons System durch Indizes a oder b gekennzeichnet.

Die Schwingungen des Benzols lassen sich in CH- und CC-Schwingungen unterteilen, wobei letztere als Gerüstschwingungen aufzufassen sind. Für eine äußerst anschauliche dynamische Darstellung der Benzolnormalmoden sei auf [61] verwiesen. Die CH-Streckschwingungen $\nu_{1-6}$ stellen die höchstfrequente Gruppe von Moden dar, ihre Frequenzen liegen im für Aromaten typischen Bereich von 3000 bis $3100 \mathrm{~cm}^{-1}$. Weitere $\mathrm{CH}$-Schwingungen sind die Biegeschwingungen innerhalb der Molekülebene $\left(\nu_{11}\right.$ und $\left.\nu_{13-17}\right)$ mit Energien zwischen 1400 und $1000 \mathrm{~cm}^{-1}$, sowie die Biegeschwingungen $\nu_{20-26}$, in denen die Auslenkung senkrecht zur Molekülebene erfolgt. Die restlichen Moden zählen zu den Gerüstschwingungen und lassen sich als CC-Streckschwingungen $\left(\nu_{7-10}, \nu_{12}, \nu_{19}\right)$ oder CC-Biegeschwingungen klassifizieren. Letztere bewirken eine Deformation des aromatischen Gerüstes parallel zur Molekülebene $\left(\nu_{18}, \nu_{27,28}\right)$ oder eine Verdrillung aus der Ebene heraus $\left(\nu_{20}, \nu_{29,30}\right)$.

$\nu_{1}$ bildet als einzige totalsymmetrische Mode [1:2]-Resonanzen (FermiResonanzen, [62]) mit den ersten Obertönen der CC-Streckschwingungen $\nu_{7}-\nu_{10}$ aus. Resonanzen sind dann symmetrieerlaubt, wenn das direkte Produkt aller beteiligten Moden die totalsymmetrische Darstellung der Punktgruppe - in diesem Fall $\mathrm{A}_{1 \mathrm{~g}}$ - enthält. Eine intensive [1:(1+1)]-Resonanz liegt somit zwischen der Fundamentalen $\nu_{7}$ und dem Kombinationston $\nu_{19}+\nu_{27}$ bei $1600 \mathrm{~cm}^{-1}$ vor. Isoenergetisch ist ebenfalls die CH-Schwingung $\nu_{2}$ mit den Kombinationen $\nu_{9}+\nu_{19}+\nu_{27}$ und $\nu_{7}+\nu_{9}$.

Derartige Resonanzen führen zu einer Verschiebung der betroffenen Energieniveaus gegenüber ihren ungestörten Werten und erschweren die Schwingungsanalyse von IR- und Raman-Spektren. Zudem müssen bei der folgenden Analyse Anharmonizitäten berücksichtigt werden, daher sind die hier dokumentierten $\mathrm{Zu}$ ordnungen als Vorschläge zu betrachten. 


\section{Helle Zustände im Experiment}

Abbildung 4.1 zeigt das IR-Absorptionsspektrum von Benzol bei Wellenlängen zwischen 1500 und $2300 \mathrm{~nm}$, die einem Energiebereich von 4300-6200 $\mathrm{cm}^{-1}$ entsprechen. Es sind zwei Gruppen von Absorptionsbanden um $1670 \mathrm{~nm}\left(6000 \mathrm{~cm}^{-1}\right)$ bzw. $2150 \mathrm{~nm}\left(4600 \mathrm{~cm}^{-1}\right)$ erkennbar, die beide im Experiment angeregt wurden. Aus der Analyse dieser Banden läßst sich die Natur des jeweiligen mit dem IRPump-Puls präparierten hellen Zustands nullter Ordnung herleiten.

Spektrale Signaturen um $6000 \mathrm{~cm}^{-1}$ werden i. A. den Obertönen von $\mathrm{CH}-$ Streckschwingungen zugeordnet. In diesem Fall muß die Natur des angeregten hellen Zustandes jedoch genauer erörtert werden. Die Auswahlregeln für die Punktgruppe $\mathrm{D}_{6 \mathrm{~h}}$ besagen, daß hier nur nur zwei Arten von Infrarotübergängen möglich sind. Liegt das elektrische Übergangsdipolmoment in der Molekülebene, sind senkrechte Übergänge zu Schwingungszuständen mit $\mathrm{E}_{1 \mathrm{u}}$-Symmetrie erlaubt, während mit einem elektrischen Dipolmoment außerhalb der Molekülebene Zustände mit $\mathrm{A}_{1 \mathrm{u}}$-Symmetrie erreicht werden können. Die ungestörten $\mathrm{CH}-$ Streckschwingungen fallen in die erste Kategorie. Somit ist nur eine der vier CH-Streckfundamentalen IR-aktiv $\left(\nu_{2,3}\right)$. Gerade Obertöne $(v=2,4, \ldots)$ sämtlicher Normalmoden des Benzols besitzen $\mathrm{A}_{1 \mathrm{~g}}$-Symmetrie und sind somit Raman-aktiv, als IR-Übergänge jedoch verboten, was sich direkt auf die Existenz eines Inversionszentrums im Molekül zurückführen läßt. Folglich ist der Bereich um $6000 \mathrm{~cm}^{-1}$ im IR-Spektrum nicht Ober-, sondern Kombinationstönen von CH-Streckschwingungen zuzuordnen. Nur drei der denkbaren binären Kombinationen besitzen Komponenten der geforderten $\mathrm{E}_{1 \mathrm{u}}$-Darstellung, und zwar $\nu_{2,3}+\nu_{5,6}, \nu_{1}+\nu_{2,3}$ und $\nu_{4}+\nu_{5,6}$. Iachello und Oss ordneten des weiteren die schwächeren Banden zwischen $1675 \mathrm{~nm}\left(5975 \mathrm{~cm}^{-1}\right)$ und $1680 \mathrm{~nm}\left(5950 \mathrm{~cm}^{-1}\right)$ den Kombinationstönen $\nu_{2}+\nu_{13}+\nu_{15}+\nu_{27}\left(5998 \mathrm{~cm}^{-1}\right)$ sowie $\nu_{2}+\nu_{7}+\nu_{12}\left(5973 \mathrm{~cm}^{-1}\right)$ $\mathrm{zu}[63]$.

Der Vollständigkeit halber sei an dieser Stelle die alternative Beschreibung von Molekülschwingungen mit dem Modell der Lokalmoden erwähnt. Durch Linearkombination stark anharmonisch gekoppelter Normalmoden kann eine Schwingung beschrieben werden, bei der die gesamte Energie in einem Oszillator lokalisiert ist. Benzol besäße in diesem Sinne $6 \mathrm{CH}$-Oszillatoren, die bei steigender CH-Obertonanregung $(v=2,3,4 \ldots)$ sowohl sukzessive aktiviert als auch einzeln mehrfach angeregt werden könnten. Allerdings konnten Lee et al. zeigen, daß die $\mathrm{CH}-$ Streckschwingung mit mindestens drei Quanten populiert sein muß, um die 


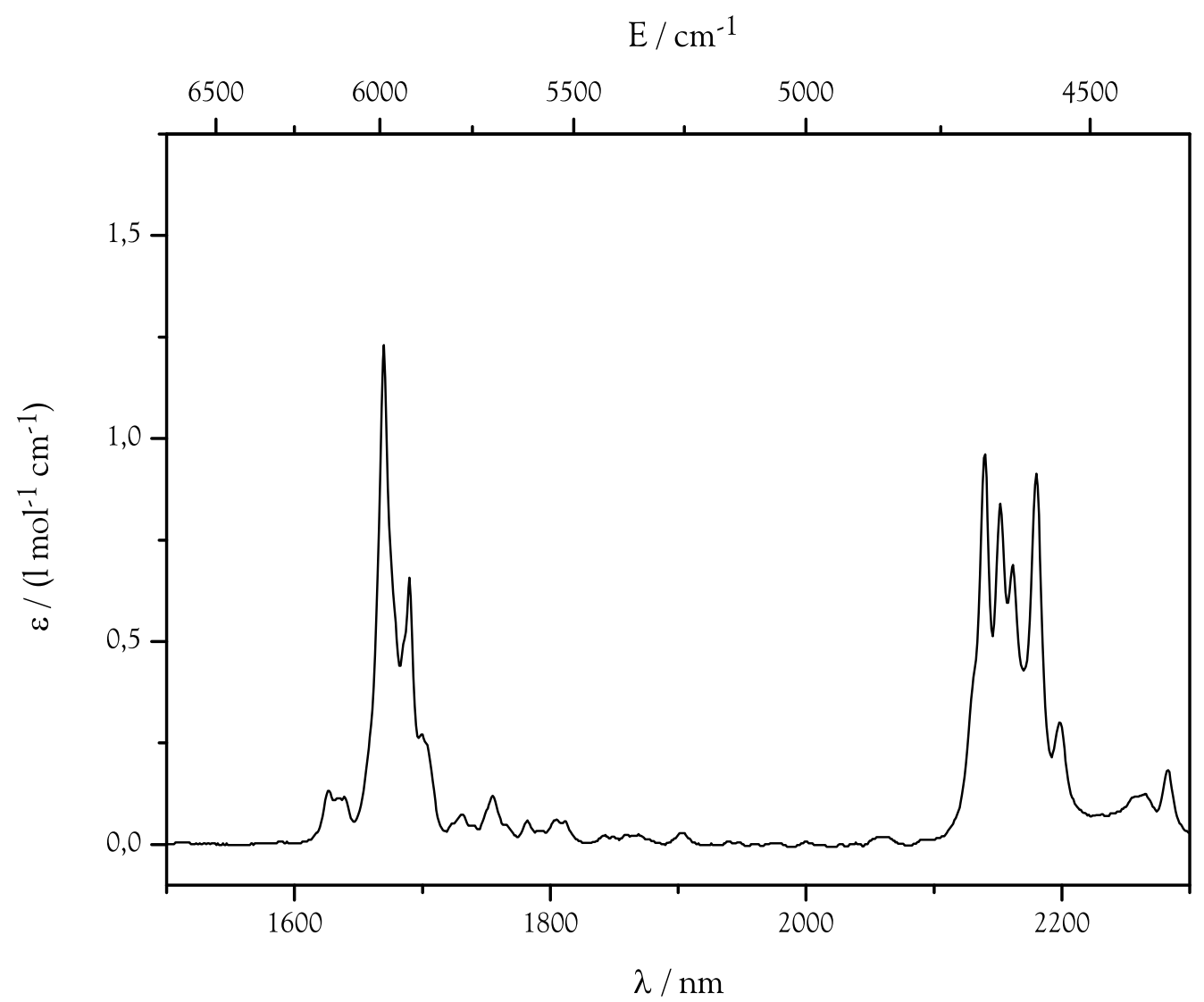

Abbildung 4.1: nIR-Absorptionsspektrum des Benzols

hochaufgelösten Obertonspektren mit dem Lokalmodenmodell ansatzweise reproduzieren zu können [64]. Doch selbst dann ist ein reiner Lokalmodenansatz nicht zufriedenstellend, u. a. weil in theoretischen und experimentellen Studien gezeigt werden konnte, daß für alle CH-Streckmoden ausgeprägte Fermi-Resonanzen mit $\mathrm{CH}$-Biegeschwingungen geeigneter Symmetrie existieren [65,66]. Im Rahmen dieser Arbeit wird der helle Zustand des Benzols bei $6000 \mathrm{~cm}^{-1}$ daher als $v_{\mathrm{CH}}=2$ bezeichnet.

Die Banden des Spektrums um $2170 \mathrm{~nm}\left(4600 \mathrm{~cm}^{-1}\right)$ rühren dagegen vorwiegend von Übergängen zu Kombinationstönen der Moden $\nu_{1}-\nu_{6}$ mit $\nu_{7}-\nu_{12}$ her. Symmetrieerlaubt sind z. B. $\nu_{1}+\nu_{9}\left(2194 \mathrm{~nm}, 4556 \mathrm{~cm}^{-1}\right)$ und $\nu_{4}+\nu_{7}(2147 \mathrm{~nm}$, 
Kapitel 4 Modellsysteme

$\left.4657 \mathrm{~cm}^{-1}\right)$. Ebenfalls als optischer Übergang im Infraroten erlaubt sind [1:2]Kombinationen der CC-Streckschwingungen wie $2 \nu_{7}+\nu_{9}$ bei $2135 \mathrm{~nm}\left(4683 \mathrm{~cm}^{-1}\right)$.

Durch Wahl einer geeigneten IR-Pumpwellenlänge können unter diesen Voraussetzungen selektiv Kombinationstöne von CC- mit CH-Schwingungen $(2140 \mathrm{~nm}$, $\left.4673 \mathrm{~cm}^{-1}\right)$ oder der CH-Streckschwingungen $\left(1670 \mathrm{~nm}, 5988 \mathrm{~cm}^{-1}\right)$ angeregt werden.

\section{FC-aktive Moden}

Die Observable des Experiments ist die Änderung der Absorption eines ultravioletten Probepulses, der den ersten elektronischen Übergang im Modellsystem initiiert (s. Abschn. 3.1). Wie bereits angedeutet (S. 29), verbieten die Auswahlregeln für die Punktgruppe $\mathrm{D}_{6 \mathrm{~h}}$ den rein elektronischen $\left(v^{\prime}=0, v^{\prime \prime}=0\right)$-Übergang ${ }^{1}$ von $\mathrm{S}_{0}$ nach $\mathrm{S}_{1}$. Vibronische Übergänge, die mit der Änderung der Besetzung bestimmter Normalmoden geeigneter Symmetrie einhergehen, sind hingegen erlaubt. Diese als Franck-Condon(FC)-aktiv bezeichneten Schwingungen können anhand von UV-Absorptionsdaten identifiziert werden.

Das Absorptionsspektrum des ersten elektronischen Übergangs von Benzol wurde erstmals von Turkevich et al. hochaufgelöst präsentiert [67]. Es handelt sich um einen ${ }^{1} \mathrm{~B}_{2 u} \leftarrow{ }^{1} \mathrm{~A}_{1 \mathrm{~g}}$-Übergang. Callomon et al. führten eine nahezu vollständige Schwingungs- und Rotationsanalyse dieses sog. $2600 \AA$-Systems durch [68], welche als Grundlage der folgenden FC-Moden-Bestimmung dient. Das Spektrum weist eine Vielzahl von Progressionen auf, die eine eindeutige Zuordnung der zugrundeliegenden Schwingungsübergänge ermöglichen. So sind die intensivsten Übergänge auf der kurzwelligen Flanke des theoretischen (0,0)-Übergangs Kombinationsanregungen gemäß $27_{0}^{1}+19_{0}^{n}(n=1,2, \ldots)$ zuzuschreiben ${ }^{2}$. Dies kann als (1,0)-Übergang von $\nu_{27}$ interpretiert werden, von dem Intensität an die eigentlich symmetrieverbotene Mode $\nu_{19}$ abgegeben wird. Dies ist leicht verständlich, wenn der Charakter von $\nu_{19}$ (symmetrische Ringatmung) und die Geometrie des $\mathrm{S}_{1}$-Benzols (regelmäßiges Sechseck mit leicht gestreckten CC-Bindungen und verkürzten CH-Abständen [68]) verglichen werden.

Der im Experiment eingesetzte UV-Abfragepuls liegt mit $270 \mathrm{~nm}$ deutlich auf der roten Seite des $(0,0)$-Übergangs. In dem von der spektralen Pulsbreite erfaßten

${ }^{1}$ Der Konvention entsprechend bezeichnet $v^{\prime}$ die Schwingungsquantenzahl des elektronisch angeregten Zustands und $v^{\prime \prime}$ die des elektronischen Grundzustandes.

${ }^{2}$ In der Notation $\nu_{a}^{b}$ steht $a$ für $v^{\prime \prime}(\nu)$ und $b$ für $v^{\prime}(\nu)$. 
Bereich zeigt das Gasphasenspektrum die ersten zwei heißen Banden $(v=1,2)$ der Mode $\nu_{27}$, deren Intensitätsverhältnis grob die Boltzmannbesetzung widerspiegelt. Der breite Untergrund der langwelligen Flanke enthält außerdem noch eine Bande, die dem Übergang $29_{2}^{0}$ zuzuordnen ist, aber nur gering ausgeprägt ist.

Demzufolge stellen die FC-aktiven Moden im Experiment hauptsächlich niederfrequente Gerüstschwingungen dar, von denen die in-plane-Ringdeformation $\nu_{27}\left(608 \mathrm{~cm}^{-1}\right.$, Wilsonnr. 6) den überwiegenden Anteil zum Signal beisteuern sollte. Beiträge der Moden $\nu_{26}$ und $\nu_{29}$ sind möglichweise ebenfalls in geringem Maße vorhanden. In jedem Fall gehören die möglichen FC-aktiven Schwingungen zu den niederfrequentesten der 30 Normalmoden. Dadurch eignet sich dieses System hervorragend für die Untersuchung des gesamten IVR-Prozesses mit Hilfe der IR-Pump-UV-Probe-Spektroskopie, da die abgefragten Moden im statistischen Mittel vermehrt am Ende der Relaxationskaskade populiert werden (siehe Abschn. 2.1).

Abb. 4.2 stellt im oberen Teil die temperaturabhängigen Absorptionsspektren im Bereich des ersten elektronischen Übergangs für Benzol dar. Das Temperaturinkrement zwischen aufeinanderfolgenden Spektren beträgt ca. $50^{\circ} \mathrm{C}$. Trotz geringer Auflösung ist in dem Spektrum bei $68^{\circ} \mathrm{C}$ (grau hinterlegt) die oben beschriebene Progressionsstruktur deutlich zu erkennen. Mit steigender Temperatur nimmt die Fläche unter der gesamten Absorptionsbande kontinuierlich zu, ein isospestischer Punkt kann nicht definiert werden. Dies ist auf die zunehmende Störung der $\mathrm{D}_{6 \mathrm{~h}}$-Symmetrie zurückzuführen, wodurch das Verbot für diesen Übergang generell geschwächt wird.

Über die statistische Thermodynamik kann die Beziehung zwischen optischer Dichte und mittlerer Innerer Energie aufgestellt werden. Die Temperaturabhängigkeit der Inneren Energie wird durch

$$
E(T)=\sum_{i=0}^{N} \frac{\tilde{\nu}_{i}}{\exp \left(\tilde{\nu}_{i} / k T\right)-1}
$$

mit $\quad N$ : Anzahl der Normalmoden

$\tilde{\nu}_{i}$ : Energie der Normalmoden in $\mathrm{cm}^{-1}$

$k$ : Boltzmann-Konstante 

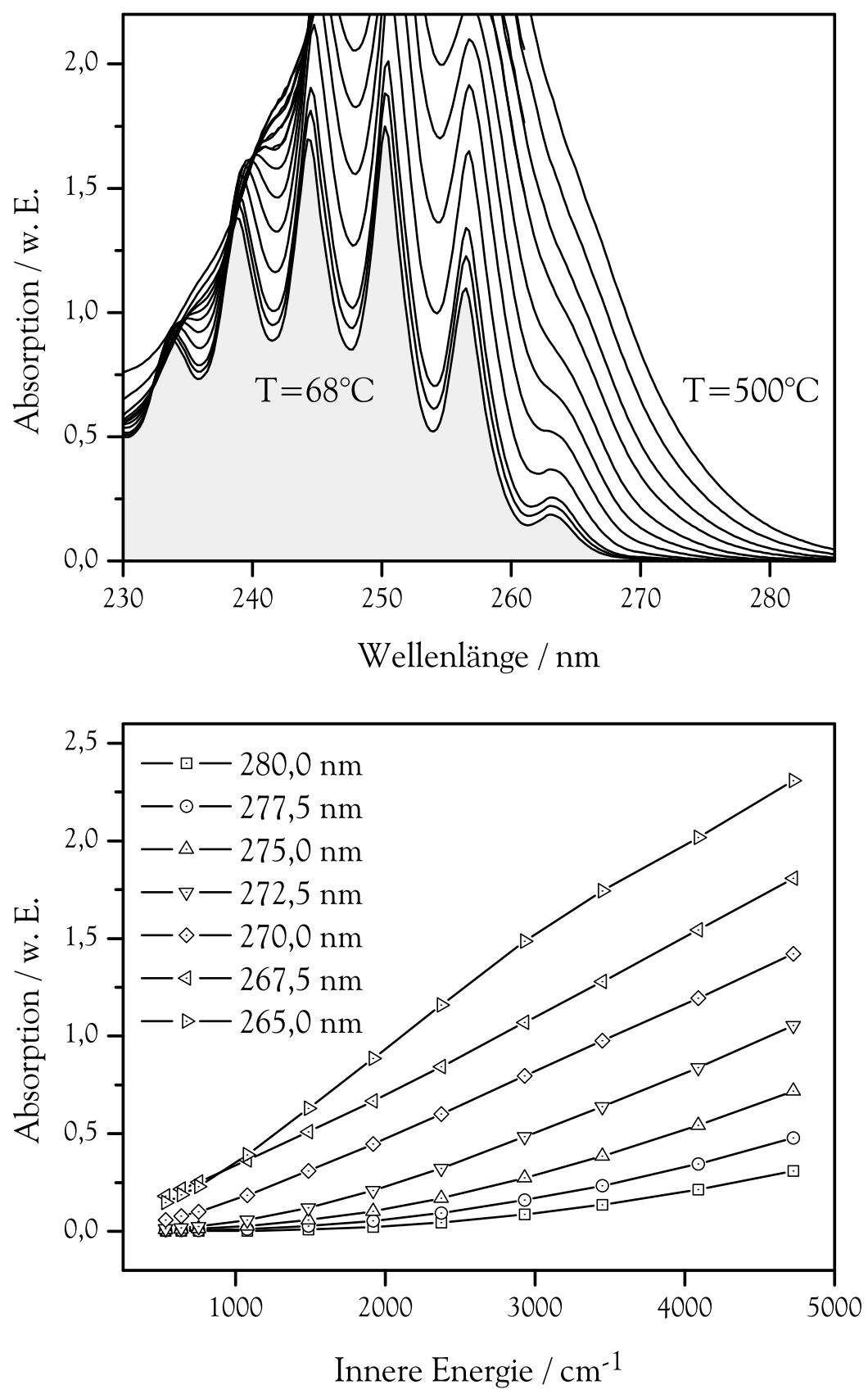

Abbildung 4.2: Temperaturabhängige UV-Absorptionsspektren des Benzols (oben) und daraus resultierende Energieabhängigkeit der Absorption (unten) 
festgelegt. Hiermit können die Absorptionen einzelner Wellenlängen auf der roten Flanke des (0,0)-Übergangs gegen die Innere Energie aufgetragen werden, wie in der unteren Hälfte von Abb. 4.2 geschehen. Es liegt demzufolge eine nahezu lineare Energieabhängigkeit des Absorptionskoeffizienten bei der Probewellenlänge $(270 \mathrm{~nm})$ vor. Die Anpassung der Eichkurven mit einem Polynom 3. Grades ist in Anhang A.1 zu finden.

\subsection{Benzol- $\mathrm{d}_{1}$}

Die 30 Normalmoden des $\mathrm{C}_{6} \mathrm{H}_{5} \mathrm{D}$ bilden die Darstellungen $11 \mathrm{a}_{1}+3 \mathrm{a}_{2}+6 \mathrm{~b}_{1}+10 \mathrm{~b}_{2}$ in der Punktgruppe $\mathrm{C}_{2 \mathrm{v}}$. Wird das Koordinatensystem so gewählt, daß die x-Achse senkrecht zur Molekülebene verläuft, beschreiben die $a_{1}$ - und $b_{2}$-Darstellungen Schwingungen in der Molekülebene. Die Auswahlregeln erlauben im IR-Spektrum nur Übergänge der $a_{1^{-}}, b_{1^{-}}$und $b_{2}$-Darstellungen, wohingegen alle Schwingungen Raman-aktiv sind. Eine sehr genaue Zuordnung der 30 Fundamentalfrequenzen anhand von Frequenzberechnungen, polarisierten Ramanspektren und IRSpektren von gasförmigem und flüssigen Deuterobenzol wurde von J. E. Bertie et al. vorgenommen [69].

Die Monodeuterierung des Benzols bewirkt eine Abnahme der molekularen Symmetrie, ohne die übrigen Eigenschaften des Moleküls stark zu verändern. Dies ist deutlich an den Frequenzen der 30 Normalmoden von Benzol- $\mathrm{d}_{1}$ zu sehen, die in Tab. 4.2 nach absteigender Energie sortiert aufgeführt sind. Auffälligstes Resultat der Symmetriereduktion ist die Aufspaltung vorher entarteter Schwingungsenergien. Darüber hinaus zeigt der Großteil der Moden im substituierten Molekül jedoch nur geringe Änderungen ihrer Frequenzen, wie aus einem Vergleich mit Tab. 4.1 unter Einbeziehung der Wilsonnomenklatur schnell ersichtlich wird. Lediglich vier Schwingungen weichen stark in ihrer Frequenz vom Benzolwert ab. So ist von den sechs CH-Streckschwingungen die Mode $\nu_{6}$ (Wilsonnr. 13) um $800 \mathrm{~cm}^{-1}$ verringert und liegt nun als einzige Schwingung bei $2300 \mathrm{~cm}^{-1}$ vor. Diese Mode stellt im unsubstituierten Benzol eine reine CH-Streckschwingung dar, bei der in einer halben Schwingungsperiode die CH-Bindungen umlaufend um den Ring alternierend gestreckt bzw. gestaucht werden. Die große Abnahme der Frequenz infolge der Monodeuterierung ist direkt auf die kleinere Kraftkonstante der CD-Bindung zurückzuführen. Ebenfalls in ihrer Frequenz um $200 \mathrm{~cm}^{-1}$ verringert ist $\nu_{22}$ (Wilson-Nr. 9b). Es handelt sich hierbei um eine CH-Biegeschwingung, bei 
der das D-Atom (Position 1) sowie die H-Atome in Position 3, 4 und 6 große Bewegungsamplituden parallel zur Molekülebene besitzen. Die korrespondierende Wilson-Mode 9a $\left(\nu_{13}\right)$ bleibt dagegen von der Deuterierung unbeeinflußt, da hier nur die H-Atome in Position 2, 3, 5 und 6 eine Auslenkung erfahren. Eine Abnahme der Frequenz um jeweils ca. $70 \mathrm{~cm}^{-1}$ zeigen schließlich noch die Moden $\nu_{24}$ (10b) und $\nu_{27}$ (11), welche beide als Biegeschwingungen der H/D-Atome senkrecht zur Molekülebene bei näherungsweise starrer Gerüstgeometrie aufgefaßt werden können.

Da die Frequenzen der übrigen Moden näherungsweise erhalten bleiben, dürfen die Schlußfolgerungen über intramolekulare Kopplungen, die für das Benzol getroffen wurden, übertragen werden. So sind z. B. auch für Benzol- $\mathrm{d}_{1}$ ausgeprägte Fermiresonanzen zwischen den $\mathrm{CH}-S t r e c k-$ und in-plane-CH-Biegeschwingungen zu erwarten.

\begin{tabular}{|cccc|cccc|}
\hline Mode & $\begin{array}{c}\text { Energie } \\
{\left[\mathbf{c m}^{-1}\right]}\end{array}$ & Sym. & Wnr. & Mode & $\begin{array}{c}\text { Energie } \\
{\left[\mathbf{c m}^{-\mathbf{1}}\right]}\end{array}$ & Sym. & Wnr. \\
\hline \hline$\nu_{1}$ & 3078 & $\mathrm{~b}_{2}$ & $20 \mathrm{~b}$ & $\nu_{16}$ & 1032 & $\mathrm{a}_{1}$ & $18 \mathrm{a}$ \\
$\nu_{2}$ & 3075 & $\mathrm{a}_{1}$ & $20 \mathrm{a}$ & $\nu_{17}$ & 1006 & $\mathrm{a}_{1}$ & 12 \\
$\nu_{3}$ & 3070 & $\mathrm{a}_{1}$ & $7 \mathrm{a}$ & $\nu_{18}$ & 1001 & $\mathrm{~b}_{1}$ & 5 \\
$\nu_{4}$ & 3053 & $\mathrm{a}_{1}$ & 2 & $\nu_{19}$ & 994 & $\mathrm{a}_{1}$ & 1 \\
$\nu_{5}$ & 3043 & $\mathrm{~b}_{2}$ & $7 \mathrm{~b}$ & $\nu_{20}$ & 979 & $\mathrm{a}_{2}$ & $17 \mathrm{a}$ \\
$\nu_{6}$ & 2282 & $\mathrm{a}_{1}$ & 13 & $\nu_{21}$ & 944 & $\mathrm{~b}_{1}$ & $17 \mathrm{~b}$ \\
$\nu_{7}$ & 1595 & $\mathrm{a}_{1}$ & $8 \mathrm{a}$ & $\nu_{22}$ & 859 & $\mathrm{~b}_{2}$ & $9 \mathrm{~b}$ \\
$\nu_{8}$ & 1591 & $\mathrm{~b}_{2}$ & $8 \mathrm{~b}$ & $\nu_{23}$ & 853 & $\mathrm{a}_{2}$ & $10 \mathrm{a}$ \\
$\nu_{9}$ & 1474 & $\mathrm{a}_{1}$ & $19 \mathrm{a}$ & $\nu_{24}$ & 784 & $\mathrm{~b}_{1}$ & $10 \mathrm{~b}$ \\
$\nu_{10}$ & 1451 & $\mathrm{~b}_{2}$ & $19 \mathrm{~b}$ & $\nu_{25}$ & 692 & $\mathrm{~b}_{1}$ & 4 \\
$\nu_{11}$ & 1327 & $\mathrm{~b}_{2}$ & 3 & $\nu_{26}$ & 606 & $\mathrm{a}_{1}$ & $6 \mathrm{a}$ \\
$\nu_{12}$ & 1296 & $\mathrm{~b}_{2}$ & 14 & $\nu_{27}$ & 605 & $\mathrm{~b}_{1}$ & 11 \\
$\nu_{13}$ & 1172 & $\mathrm{a}_{1}$ & $9 \mathrm{a}$ & $\nu_{28}$ & 604 & $\mathrm{~b}_{2}$ & $6 \mathrm{~b}$ \\
$\nu_{14}$ & 1157 & $\mathrm{~b}_{2}$ & 15 & $\nu_{29}$ & 400 & $\mathrm{a}_{2}$ & $16 \mathrm{a}$ \\
$\nu_{15}$ & 1080 & $\mathrm{~b}_{2}$ & $18 \mathrm{~b}$ & $\nu_{30}$ & 378 & $\mathrm{~b}_{1}$ & $16 \mathrm{~b}$ \\
\hline
\end{tabular}

Tabelle 4.2: Die 30 Normalschwingungen des Benzol- $d_{1}$, nach absteigender Energie sortiert [69,70]; Wnr.: Wilson-Nummer 


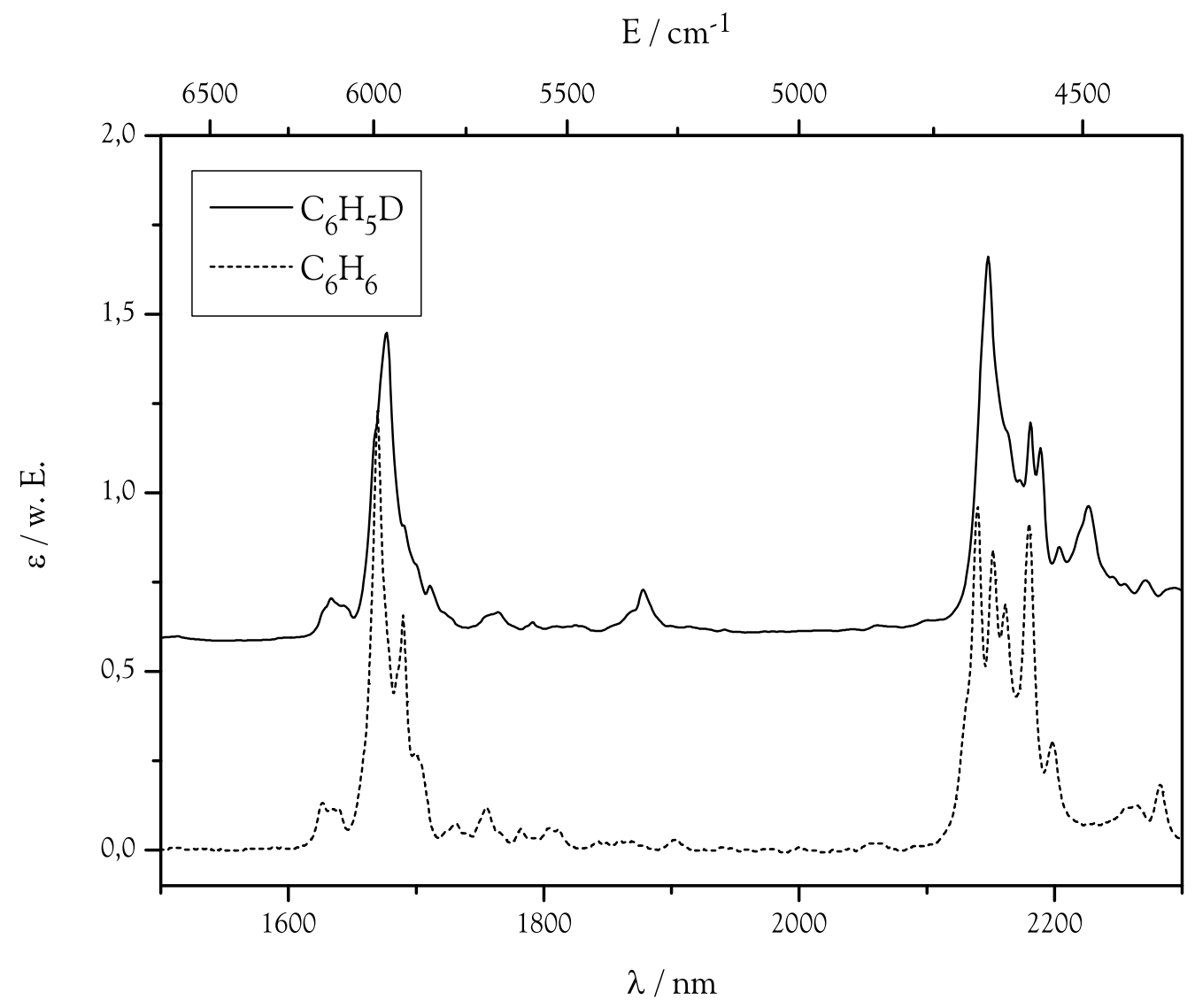

Abbildung 4.3: nIR-Absorptionsspektrum des Monodeutero-Benzols

\section{Helle Zustände im Experiment}

In Abb. 4.3 sind die nIR-Absorptionsspektren von unsubstituiertem und monodeuteriertem Benzol in verdünnter 1,1,2-Trichlortrifluorethan-Lösung gegenübergestellt. Die beiden Substanzen zeigen eine auffallend ähnliche Struktur ihrer Spektren. Es ist somit zu erwarten, daß auch die im Experiment präparierten hellen Zustände des $\mathrm{C}_{6} \mathrm{H}_{5} \mathrm{D}$ denen des $\mathrm{C}_{6} \mathrm{H}_{6}$ entsprechen. Die Zuordnung der $\mathrm{C}_{6} \mathrm{H}_{5} \mathrm{D}$ Banden wird erheblich dadurch erleichtert, daß für nahezu alle Kombinations- und Obertöne von Monodeuterobenzol die beobachteten Wellenzahlen mit der Summe der Fundamentalfrequenzen übereinstimmen. Lediglich im Bereich des ersten 
Kapitel 4 Modellsysteme

Obertons der CH- und CD-Streckschwingung gestaltet sich die Analyse etwas schwieriger.

Im Gegensatz zum Benzol mit $\mathrm{D}_{6 \mathrm{~h}}$-Punktgruppe sind im monodeuterierten Derivat auch die geraden Obertöne aller Schwingungen parallel zur Molekülebene IR-aktiv, da das entsprechende Übergangsdipolmoment in der Punktgruppe $\mathrm{C}_{2 \mathrm{v}}$ totalsymmetrisch transformiert. Somit wird die breite Bande mit Maximum bei $1677 \mathrm{~nm}\left(5963 \mathrm{~cm}^{-1}\right)$ hauptsächlich durch die Zustände $2 \nu_{1-5}$ verursacht. Die Rotverschiebung um ca. $100 \mathrm{~cm}^{-1}$ kann auf die bei CH-Streck-Obertönen auftretenden Anharmonizitäten zurückgeführt werden. Darüber hinaus tragen auch hier CH-Streck-Kombinationstöne, wie sie beim Benzol dominieren, zur Absorption bei. Im breiten Untergrund dieser Bande liegen schließlich noch Kombinationstöne aus drei und mehr Moden, welche zu zahlreich sind, um eine eindeutige $\mathrm{Zu}-$ ordnung zuzulassen [69]. Es ist jedoch anzunehmen, daß CH-Streckoszillationen mit $v=2$ den Charakter des hellen Zustands nullter Ordnung definieren, wenn dieser mit einem IR-Anregungspuls der Wellenlänge $1677 \mathrm{~nm}$ präpariert wird. Die CD-Streckschwingung ist in diesem spektralen Bereich des Spektrums vernachlässigbar.

Der zweite Komplex von Banden im $\mathrm{C}_{6} \mathrm{H}_{5} \mathrm{D}$-Spektrum besitzt ein Maximum bei $2148 \mathrm{~nm}\left(4655 \mathrm{~cm}^{-1}\right)$, welches primär von Kombinationstönen der CH-Streckschwingungen $\nu_{2-4}$ mit den CC-Streckschwingungen $\nu_{7}$ und $\nu_{8}$ verursacht wird. Die beiden Maxima bei 2181 bzw. $2189 \mathrm{~nm}$ sind höheren Kombinationsbanden zuzuschreiben, die allerdings aufgrund der Vielzahl von möglichen symmetrieerlaubten Modenkombinationen nicht genauer identifiziert werden können. Eine weitere Bande bei $2226 \mathrm{~nm}\left(4492 \mathrm{~cm}^{-1}\right)$, die im Benzolspektrum nicht erscheint, kann dem ersten Oberton der CD-Streckschwingung zugeordnet werden. Eine Anregung dieses Zustands fand im Experiment jedoch nicht statt, da die spektrale Breite des Pumppulses bei $2148 \mathrm{~nm}$ diese Bande nicht mit einschließt.

\section{FC-aktive Moden}

Eine detaillierte Analyse des $\mathrm{S}_{1} \leftarrow \mathrm{S}_{0}$-Übergangs wie für Benzol in [68] existiert für das monodeuterierte Derivat nicht. Da jedoch, wie in den vorigen Abschnitten dargelegt, die spektroskopischen Eigenschaften des Muttermoleküls von der Substitution überwiegend unbeeinflußt bleiben, ist auch im Falle des UVAbsorptionsspektrums ein direkter Vergleich von $\mathrm{C}_{6} \mathrm{H}_{6}$ und $\mathrm{C}_{6} \mathrm{H}_{5} \mathrm{D}$ zulässig. Daher sind in Abb. 4.4 die Spektren von Benzol, verdünnt in Tetrachlormethan, 


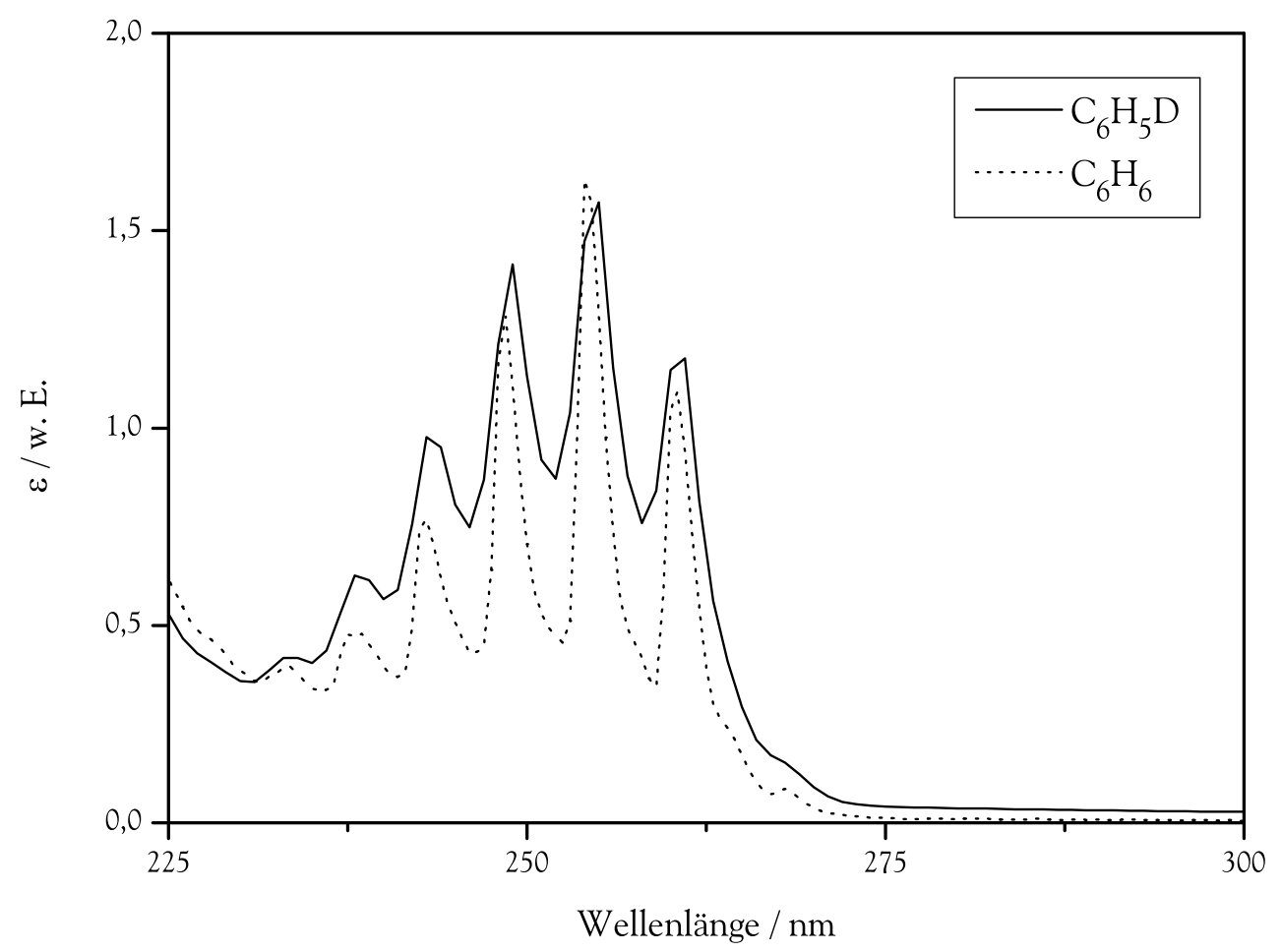

Abbildung 4.4: UV-Absorptionsspektrum des verdünnten Monodeuterobenzols

und Monodeuterobenzol, verdünnt in Trichlortrifluorethan, gegenübergestellt. Es liegt offensichtlich die gleiche Progressionsstruktur vor, welche im Falle des Benzols lediglich etwas stärker ausgeprägt ist. Auch konnte gezeigt werden, daß in $\mathrm{C}_{5} \mathrm{H}_{6} \mathrm{D}$ der Beginn der Progression von $27 \mathrm{a}, \mathrm{b}_{0}^{1}+19 \mathrm{a}, \mathrm{b}_{0}^{n}(n=1,2, \ldots)$ um nur $0.2 \mathrm{~nm}$ im Vergleich zum undeuterierten System verschoben ist [71]. Somit ist die Annahme, daß auch auf der langwelligen Flanke des (0,0)-Übergangs bei der Probewellenlänge $270 \mathrm{~nm}$ die benzolanalogen FC-aktiven Moden als heiße Banden absorbieren, naheliegend, zumal die Monodeuterierung auf diese Moden mit den Wilsonnummern 6 und 16 keinen großen Einfluß hat (vgl. S. 69). Die Aufhebung der Entartung geht zwar mit einer Änderung der irreduziblen Darstellung einher, doch werden auch die Auswahlregeln entsprechend relaxiert. Ein direkter Vergleich der transienten Absorptionssignale von Benzol und Benzol- $\mathrm{d}_{1}$ bei glei- 
Kapitel 4 Modellsysteme

cher Probewellenlänge ist somit zulässig. Auch die Temperaturabhängigkeit der Absorptionskoeffizienten (s. Abb. 4.2) darf als ähnlich angenommen werden.

\subsection{Chlorbenzol}

Chlorbenzol $\mathrm{C}_{6} \mathrm{H}_{5} \mathrm{Cl}$ besitzt eine zu Monodeuterobenzol isomorphe Struktur und gehört somit ebenfalls zur Punktgruppe $\mathrm{C}_{2 \mathrm{v}}$. Entsprechend faktorisieren die 30 Normalmoden des Moleküls auch hier gemäß $11 \mathrm{a}_{1}+3 \mathrm{a}_{2}+6 \mathrm{~b}_{1}+10 \mathrm{~b}_{2}$ in die irreduziblen Darstellungen dieser Symmetrieklasse. Weiterhin gelten die gleichen Auswahlregeln für optische Übergänge wie im vorhergehenden Abschnitt, so daß auch bei diesem Modellsystem lediglich Zustände mit $\mathrm{A}_{2}$-Gesamtsymmetrie nicht optisch bevölkert werden können. Die Auswirkung der Monochlorierung auf die Normalmodenfrequenzen kann anhand von Tabelle 4.3, in der die 30 Fundamentalschwingungen nach ihrer Energie sortiert sind, diskutiert werden. Hierfür erweist sich die Wilsonnomenklatur erneut als hilfreich, da sie einen Vergleich mit den Frequenzen analoger Schwingungen in Benzol (Tab. 4.1) und Benzol- $\mathrm{d}_{1}$ (Tab. 4.2) zuläßt.

Durch die Symmetriereduktion treten auch im Chlorbenzol keine entarteten Schwingungen mehr auf. Interessanterweise beeinflußt die Substitution eines Wasserstoffatoms durch das 35 mal schwerere Chloratom den Großteil der Fundamentalfrequenzen kaum, sie verschieben sich um nicht mehr als $70 \mathrm{~cm}^{-1}$. Nur die fünf Normalmoden mit den Wilsonnummern 1, 6a, 13, 10b und 15 weisen starke Frequenzänderungen auf. Die $\mathrm{CH} / \mathrm{CCl}$-Streckschwingung 13 gehört im unsubstituierten Molekül zu den sechs hochfrequentesten Moden, ist aber durch die Chlorierung um $2000 \mathrm{~cm}^{-1}$ auf $1085 \mathrm{~cm}^{-1}$ reduziert. Ein ähnlicher Effekt wurde für diese Schwingung schon beim Deuterobenzol beschrieben (s. S. 69), nur daß sich die kleinere Kraftkonstante des CCl-Oszillators noch stärker auf die Gesamtfrequenz auswirkt. Um $300 \mathrm{~cm}^{-1}$ verringert sich die Frequenz der Wilsonmode 1, die in $\mathrm{C}_{6} \mathrm{H}_{6}$ als totalsymmetrische CC-Streckschwingung unter Beibehalt der CH-Bindungslängen auftritt. Auch die a $a_{1}$-Mode Nr. 6a, in deren Verlauf der Kohlenstoffring eine Stauchung entlang der z-Achse und eine Streckung senkrecht dazu erfährt, ist um $200 \mathrm{~cm}^{-1}$ abgesenkt. Durch die Energieabnahme der Moden $15 \mathrm{um} 850 \mathrm{~cm}^{-1}$ und 10b um $650 \mathrm{~cm}^{-1}$ besitzt Chlorbenzol im Vergleich zu Benzol und Benzol- $\mathrm{d}_{1}$ schließlich zwei weitere sub- $400 \mathrm{~cm}^{-1}$-Fundamentale, wodurch die Zustandsdichte des Moleküls erheblich ansteigt (s. Abschn. 4.6). Die Wilsonmode 
$15\left(\nu_{29}\right)$ läßt sich als in-plane-Biegeschwingung der aromatischen Substituenten beschreiben, bei der das Chloratom eine relativ große Auslenkung erfährt. Dagegen bezeichnet die Wilsonnummer 10b $\left(\nu_{30}\right)$ eine out-of-plane-Biegeschwingung, die hauptsächlich die Substituenten in 1- und 4-Position betrifft.

\section{Helle Zustände im Experiment}

Im Falle des Chlorbenzols wurden nur Pump-Probe-Experimente mit einer Pumpwellenlänge im Bereich des ersten Obertons der CH-Streckschwingungen $\nu_{1-5}$ durchgeführt. Abb. 4.5 zeigt daher nur den entsprechenden Teil des nIRAbsorptionsspektrums im Bereich von ca. $5500-6500 \mathrm{~cm}^{-1}$, aufgenommen in verdünnter 1,1,2-Trichlortrifluorethanlösung. Es handelt sich um eine inhomogen verbreiterte Bande mit zwei Maxima im Verhältnis 3:2 bei 1663 und $1676 \mathrm{~nm}$. Darüber hinaus ist ein ca. $120 \mathrm{~nm}$ breiter Untergrund erkennbar. Die zweige-

\begin{tabular}{|cccc|cccc|}
\hline Mode & $\begin{array}{c}\text { Energie } \\
{\left[\mathbf{c m}^{-1}\right]}\end{array}$ & Sym. & Wnr. & Mode & $\begin{array}{c}\text { Energie } \\
{\left[\mathbf{c m}^{-\mathbf{1}}\right]}\end{array}$ & Sym. & Wnr. \\
\hline \hline$\nu_{1}$ & 3086 & $\mathrm{a}_{1}$ & $20 \mathrm{a}$ & $\nu_{16}$ & 1026 & $\mathrm{a}_{1}$ & $18 \mathrm{a}$ \\
$\nu_{2}$ & 3071 & $\mathrm{a}_{1}$ & 2 & $\nu_{17}$ & 1003 & $\mathrm{a}_{1}$ & 12 \\
$\nu_{3}$ & 3052 & $\mathrm{~b}_{2}$ & $7 \mathrm{~b}$ & $\nu_{18}$ & 985 & $\mathrm{~b}_{1}$ & 5 \\
$\nu_{4}$ & 3050 & $\mathrm{~b}_{2}$ & $20 \mathrm{~b}$ & $\nu_{19}$ & 965 & $\mathrm{a}_{2}$ & $17 \mathrm{a}$ \\
$\nu_{5}$ & 3029 & $\mathrm{a}_{1}$ & $7 \mathrm{a}$ & $\nu_{20}$ & 902 & $\mathrm{~b}_{1}$ & $17 \mathrm{~b}$ \\
$\nu_{6}$ & 1580 & $\mathrm{a}_{1}$ & $8 \mathrm{a}$ & $\nu_{21}$ & 830 & $\mathrm{a}_{2}$ & $10 \mathrm{a}$ \\
$\nu_{7}$ & 1570 & $\mathrm{~b}_{2}$ & $8 \mathrm{~b}$ & $\nu_{22}$ & 740 & $\mathrm{~b}_{1}$ & 11 \\
$\nu_{8}$ & 1477 & $\mathrm{a}_{1}$ & $19 \mathrm{a}$ & $\nu_{23}$ & 701 & $\mathrm{a}_{1}$ & 1 \\
$\nu_{9}$ & 1445 & $\mathrm{~b}_{2}$ & $19 \mathrm{~b}$ & $\nu_{24}$ & 682 & $\mathrm{~b}_{1}$ & 4 \\
$\nu_{10}$ & 1326 & $\mathrm{~b}_{2}$ & 3 & $\nu_{25}$ & 616 & $\mathrm{~b}_{2}$ & $6 \mathrm{~b}$ \\
$\nu_{11}$ & 1271 & $\mathrm{~b}_{2}$ & 14 & $\nu_{26}$ & 467 & $\mathrm{~b}_{1}$ & $16 \mathrm{~b}$ \\
$\nu_{12}$ & 1174 & $\mathrm{a}_{1}$ & $9 \mathrm{a}$ & $\nu_{27}$ & 420 & $\mathrm{a}_{1}$ & $6 \mathrm{a}$ \\
$\nu_{13}$ & 1157 & $\mathrm{~b}_{2}$ & $9 \mathrm{~b}$ & $\nu_{28}$ & 400 & $\mathrm{a}_{2}$ & $16 \mathrm{a}$ \\
$\nu_{14}$ & 1085 & $\mathrm{a}_{1}$ & 13 & $\nu_{29}$ & 297 & $\mathrm{~b}_{2}$ & 15 \\
$\nu_{15}$ & 1068 & $\mathrm{~b}_{2}$ & $18 \mathrm{~b}$ & $\nu_{30}$ & 196 & $\mathrm{~b}_{1}$ & $10 \mathrm{~b}$ \\
\hline
\end{tabular}

Tabelle 4.3: Die 30 Normalschwingungen des Chlorbenzols, nach absteigender Energie sortiert [72]. 


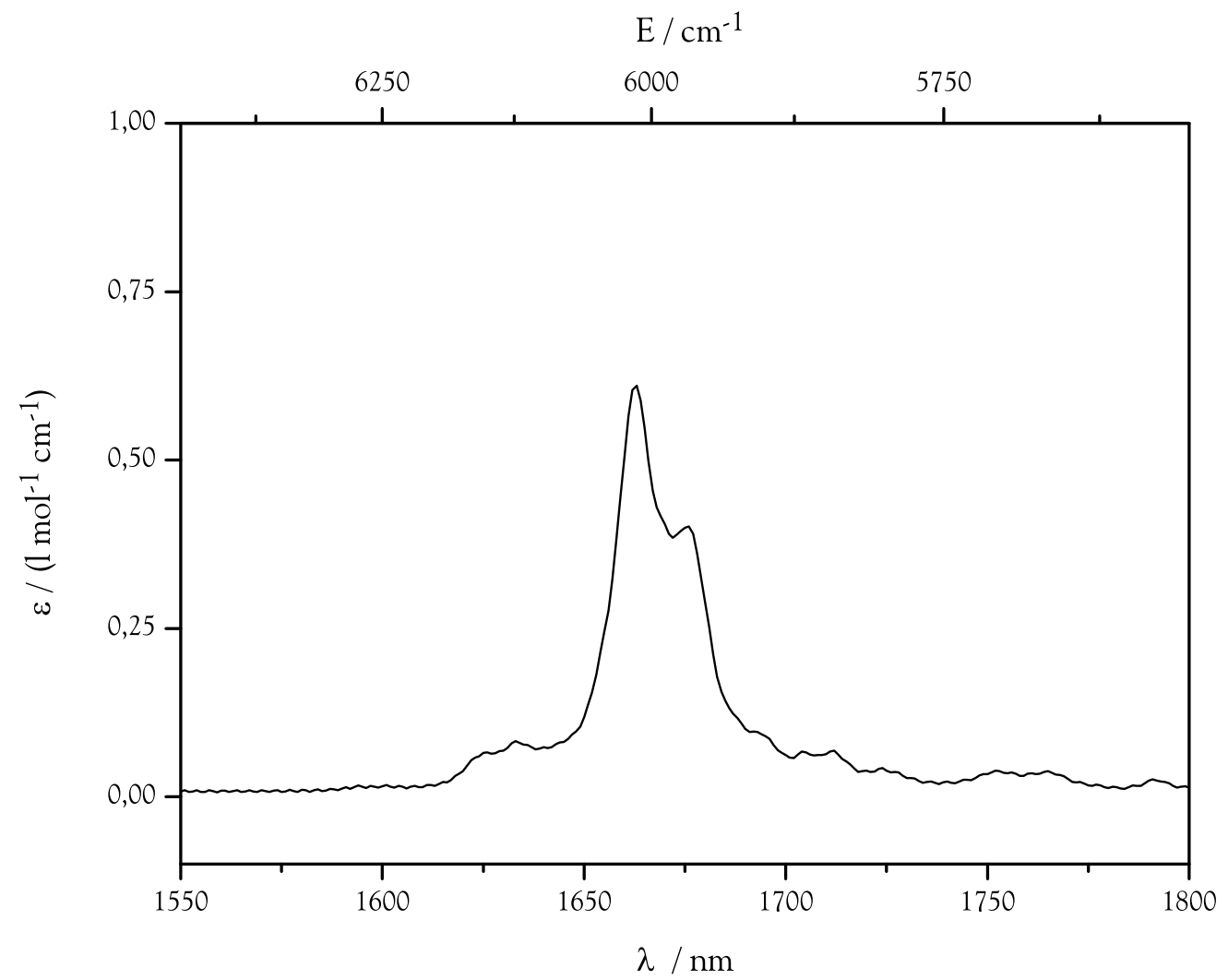

Abbildung 4.5: nIR-Absorptionsspektrum des Chlorbenzols

teilte Struktur der Bande geht bei höherer Obertonanregung $\left(v_{\mathrm{CH}}=2,3, \ldots\right)$ in einen einzigen asymmetrischen Peak über [73], was durch den ausgeprägten Lokalmodencharakter der hohen CH-Streckobertöne [74, 75] erklärt werden kann. Eine Schwingungsanalyse der $6000 \mathrm{~cm}^{-1}$-Bande existiert in der Literatur nicht; es ist allerdings zu erwarten, daß auch beim Chlorbenzol die totalsymmetrischen CH-Streckschwingungen $2 \nu_{1-5}$ den Hauptanteil an der Absorption in diesem Bereich ausmachen. Aufgrund von Anharmonizitäten ist für die reinen Obertöne eine Rotverschiebung gegenüber den Kombinationstönen der Moden $\nu_{1-5}$ wahrscheinlich. Somit ist ersteren das Absorptionsmaximum bei $6015 \mathrm{~cm}^{-1}$ $(1663 \mathrm{~nm})$ und letzteren die Schulter bei $6125 \mathrm{~cm}^{-1}$ (ca. $\left.1630 \mathrm{~nm}\right)$ zuzuordnen. In den Absorptionsbereich des zweiten Maximums bei $5968 \mathrm{~cm}^{-1}(1676 \mathrm{~nm})$ lassen 
sich Kombinationstöne der CH-Streck- mit CH-Biegeschwingungen einordnen, so z. B. $\nu_{3-5}+2 \nu_{8,9}$. Weiterhin sind Kombinationen höherer Ordnung denkbar, die jedoch eine geringere Übergangswahrscheinlichkeit besitzen und eher zum langwelligen Untergrund beitragen dürften.

Mit einem Anregungspuls der Wellenlänge $1663 \mathrm{~nm}$ wird demnach wie auch im Falle des Benzols und Monodeuterobenzols in erster Näherung ein Zustand mit $v_{\mathrm{CH}}=2$ präpariert.

\section{FC-aktive Moden}

Petruska konnte in einer Studie an mehr als 30 Benzolderivaten - darunter auch Chlorbenzol - zeigen, daß in allen Fällen die Progressionsstruktur der $\mathrm{S}_{1} \leftarrow \mathrm{S}_{0^{-}}$ Absorptionsbande des unsubstitutierten Moleküls erhalten bleibt, aber rotverschoben wird [76]. Für Chlorbenzol beträgt die Rotverschiebung $1030 \mathrm{~cm}^{-1}$. Außerdem traten in nicht-zentrosymmetrischen Systemen eine (0,0)-Bande sowie weitere Progressionsbanden auf, da in diesem Fall der Übergang optisch erlaubt ist und nicht wie beim Benzol von einer $\mathrm{e}_{2 \mathrm{~g}}$-Schwingung initiiert werden muß. Der Bandenursprung des Chlorbenzols liegt bei $37052 \mathrm{~cm}^{-1}(270 \mathrm{~nm})$ [77]. Cvitas und Hollas identifizierten in rotationskontur-aufgelösten UV-Absorptionsspektren vier heiße Banden, die sie den Moden $\nu_{23}, \nu_{25}, \nu_{27}$ und $\nu_{29}$ zuordnen. Diese $\mathrm{Zu}-$ ordnungen wurden in dispersed fluorescence-Experimenten bestätigt, mittels derer darüber hinaus die Moden $\nu_{26}, \nu_{28}$ und $\nu_{30}$ als FC-aktiv identifiziert wurden. Der im Experiment eingesetzte Probepuls mit $283 \mathrm{~nm}$ Wellenlänge liegt so weit auf der langwelligen Flanke des (0,0)-Übergangs, daß sehr wahrscheinlich auch höhere Obertöne dieser Schwingungen zur Änderung der optischen Dichte beitragen. Wieder zeigt sich die Verwandtschaft der Modellsysteme darin, daß auch im Chlorbenzol die Wilsonmoden 6a, 6b sowie 16a und 16b stark am ersten elektronischen Übergang beteiligt sind.

Die Temperaturabhängigkeit der UV-Absorption im Bereich von 250-300 nm ist im oberen Teil von Abb. 4.6 veranschaulicht. Die nullinienkorrigierten Spektren wurden zwischen 75 und $500^{\circ} \mathrm{C}$ in $25^{\circ} \mathrm{C}$-Schritten aufgezeichnet. In den kalten Spektren ist die Ursprungsbande bei $270 \mathrm{~nm}$ deutlich zu erkennen. Bei kürzeren Wellenlängen sind eine große Zahl von Progressionen und Sequenzen überlagert, so daß eine Zuordnung der Maxima bei dieser Auflösung nicht möglich ist.

Mit ansteigender Temperatur nimmt die Bandenintensität im gesamten Wellenlängenbereich zu. Der untere Graph in Abb. 4.6 zeigt Absorptionen bei ausge- 

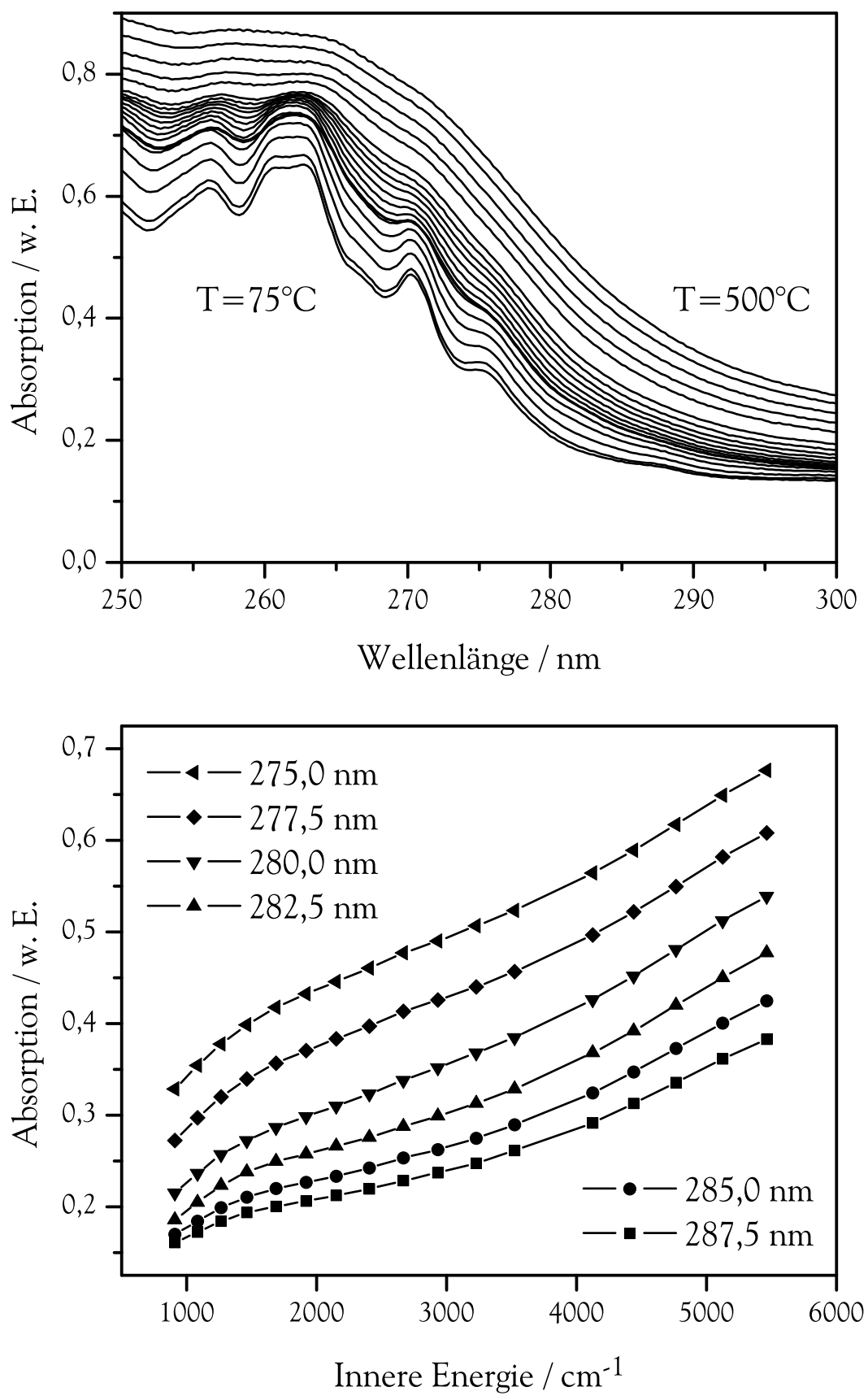

Abbildung 4.6: Temperaturabhängige UV-Absorptionsspektren des Chlorbenzols (oben) und daraus resultierende Energieabhängigkeit der Absorption (unten) 
wählten Wellenlängen in Abhängigkeit von der Inneren Energie des Chlorbenzols. Die molekülspezifische Umrechnung der Temperatur in die Innere Energie erfolgte mit Hilfe von Gl. 4.1. Die Parameter der Anpassung für $283 \mathrm{~nm}$, die in die Auswertung der transienten Absorptions-Zeit-Profile einfließen, sind in Anhang A.1 aufgeführt.

\subsection{Toluol}

Toluol $\left(\mathrm{C}_{6} \mathrm{H}_{5} \mathrm{CH}_{3}\right)$ weist als hervorstechendes Strukturmerkmal einen internen Rotator - die Methylgruppe - auf. Eine Festlegung der Molekülsymmetrie ist in diesem Fall eng an die Freiheit der Rotation gekoppelt. Im Falle stark gehinderter Rotation besitzen Moleküle des Typs $\mathrm{C}_{6} \mathrm{H}_{5} \mathrm{CY}_{3}$ lediglich eine Spiegelebene senkrecht zur Molekülebene und gehören zur Punktgruppe $\mathrm{C}_{\mathrm{s}}$. Andernfalls kann die $\mathrm{CY}_{3}$-Gruppe als Punktmasse gewertet werden und die Symmetrie erhöht sich auf $\mathrm{C}_{2 \mathrm{v}}$. Für Toluol wurde die sechsfache Barriere $V_{6}$ der inneren Rotation von Rudolph et al. zu $4.88 \mathrm{~cm}^{-1}$ bestimmt [78, 79]. Dieser niedrige Wert klassifiziert die interne Rotation als nahezu frei, womit Toluol zu den $\mathrm{C}_{5} \mathrm{H}_{6} \mathrm{X}$-Benzolderivaten $\left(\mathrm{X}=\mathrm{CH}_{3}\right)$ mit $\mathrm{C}_{2 \mathrm{v}}$-Symmetrie zu zählen ist. Als Konsequenz reduziert sich die Zahl der Schwingungsfreiheitsgrade, die rein rechnerisch $3 \mathrm{~N}-6=39$ beträgt, um die Torsionsschwingung der Methylgruppe auf 38, die sich im Verhältnis $13 \mathrm{a}_{1}+3 \mathrm{a}_{2}+13 \mathrm{~b}_{1}+9 \mathrm{~b}_{2}$ auf die irreduziblen Darstellungen der Punktgruppe aufteilen. Davon entfallen $2 a_{1}+3 b_{2}+3 b_{3}$ Schwingungen auf die Methylgruppe. Die übrigen $11 a_{1}+3 a_{2}+10 b_{1}+6 b_{2}$ Moden sind im Phenylring lokalisiert und entsprechen somit den aromatischen Schwingungen von Benzol- $\mathrm{d}_{1}$ und Chlorbenzol. Da das Bezugskoordinatensystem für die irreduziblen Darstellungen im Toluol anders gewählt wird als bei den beiden eben genannten Molekülen sind die Benennungen $b_{1}$ und $b_{2}$ vertauscht.

Tabelle 4.4 enthält eine nach abnehmender Frequenz sortierte Auflistung der Normalmoden des Toluols. Die strukturelle Ähnlichkeit zum Benzol erlaubt auch hier die Klassifizierung der Phenylschwingungen nach Wilson. Die Energien stimmen in der Gasphase und in kondensierter Phase bis auf wenige $\mathrm{cm}^{-1}$ überein [80].

Die acht Schwingungen der Methylgruppe können nicht nach Wilson bezeichnet werden. Es handelt sich hierbei um die Moden $\nu_{6}-\nu_{8}, \nu_{13}, \nu_{15}, \nu_{16}$ sowie die isoenergetischen Schwingungen $\nu_{22}$ und $\nu_{23}$. Die methylischen CH-Streckschwingungen 
$\nu_{6}-\nu_{8}$ sind von den übrigen Substituentenmoden energetisch deutlich abgesetzt und liegen im typischen Alkyl-CH-Bereich von $2900-3000 \mathrm{~cm}^{-1}$.

Daß die Interpretation des Methylsubstituenten als punktförmiges Quasiatom gerechtfertigt ist, erweist sich daran, daß sein Effekt auf die Wilsonfrequenzen 1, 10b und 15 dem des Chlorsubstituenten gleicht. Die CX-Streckschwingung 13 ist ebenfalls wieder energetisch abgesenkt, wegen der kleineren Substituentenmasse jedoch etwas schwächer als im Falle der Chlorierung.

Die acht hochfrequenten CH-Streckschwingungen des Toluols sind nahezu isoenergetisch mit Kombinations- oder Obertönen der Moden $\nu_{9-15}$. Allerdings

\begin{tabular}{|cccc|cccc|}
\hline Mode & $\begin{array}{c}\text { Energie } \\
{\left[\mathbf{c m}^{-1}\right]}\end{array}$ & Sym. & Wnr. & Mode & $\begin{array}{c}\text { Energie } \\
{\left[\mathbf{c m}^{-\mathbf{1}}\right]}\end{array}$ & Sym. & Wnr. \\
\hline \hline$\nu_{1}$ & 3087 & $\mathrm{a}_{1}$ & $7 \mathrm{a}$ & $\nu_{20}$ & 1154 & $\mathrm{~b}_{1}$ & $9 \mathrm{~b}$ \\
$\nu_{2}$ & 3063 & $\mathrm{a}_{1}$ & $20 \mathrm{a}$ & $\nu_{21}$ & 1080 & $\mathrm{~b}_{1}$ & $18 \mathrm{~b}$ \\
$\nu_{3}$ & 3055 & $\mathrm{a}_{1}$ & 2 & $\nu_{22}$ & 1040 & $\mathrm{~b}_{1}$ & - \\
$\nu_{4}$ & 3039 & $\mathrm{~b}_{1}$ & $7 \mathrm{~b}$ & $\nu_{23}$ & 1040 & $\mathrm{~b}_{2}$ & - \\
$\nu_{5}$ & 3029 & $\mathrm{~b}_{1}$ & $20 \mathrm{~b}$ & $\nu_{24}$ & 1030 & $\mathrm{a}_{1}$ & $18 \mathrm{a}$ \\
$\nu_{6}$ & 2979 & $\mathrm{~b}_{2}$ & - & $\nu_{25}$ & 1003 & $\mathrm{a}_{1}$ & 12 \\
$\nu_{7}$ & 2952 & $\mathrm{~b}_{1}$ & - & $\nu_{26}$ & 978 & $\mathrm{~b}_{2}$ & 5 \\
$\nu_{8}$ & 2921 & $\mathrm{a}_{1}$ & - & $\nu_{27}$ & 964 & $\mathrm{a}_{2}$ & $17 \mathrm{a}$ \\
$\nu_{9}$ & 1605 & $\mathrm{a}_{1}$ & $8 \mathrm{a}$ & $\nu_{28}$ & 893 & $\mathrm{~b}_{2}$ & $17 \mathrm{~b}$ \\
$\nu_{10}$ & 1586 & $\mathrm{~b}_{1}$ & $8 \mathrm{~b}$ & $\nu_{29}$ & 843 & $\mathrm{a}_{2}$ & $10 \mathrm{a}$ \\
$\nu_{11}$ & 1494 & $\mathrm{a}_{1}$ & $19 \mathrm{a}$ & $\nu_{30}$ & 784 & $\mathrm{a}_{1}$ & 1 \\
$\nu_{12}$ & 1494 & $\mathrm{~b}_{1}$ & 14 & $\nu_{31}$ & 728 & $\mathrm{~b}_{2}$ & 11 \\
$\nu_{13}$ & 1460 & $\mathrm{~b}_{1}$ & - & $\nu_{32}$ & 695 & $\mathrm{~b}_{2}$ & 4 \\
$\nu_{14}$ & 1460 & $\mathrm{~b}_{1}$ & $19 \mathrm{~b}$ & $\nu_{33}$ & 623 & $\mathrm{~b}_{1}$ & $6 \mathrm{~b}$ \\
$\nu_{15}$ & 1460 & $\mathrm{~b}_{2}$ & - & $\nu_{34}$ & 521 & $\mathrm{a}_{1}$ & $6 \mathrm{a}$ \\
$\nu_{16}$ & 1379 & $\mathrm{a}_{1}$ & - & $\nu_{35}$ & 464 & $\mathrm{~b}_{2}$ & $16 \mathrm{~b}$ \\
$\nu_{17}$ & 1312 & $\mathrm{~b}_{1}$ & 3 & $\nu_{36}$ & 408 & $\mathrm{a}_{2}$ & $16 \mathrm{a}$ \\
$\nu_{18}$ & 1208 & $\mathrm{a}_{1}$ & 13 & $\nu_{37}$ & 344 & $\mathrm{~b}_{1}$ & 15 \\
$\nu_{19}$ & 1175 & $\mathrm{a}_{1}$ & $9 \mathrm{a}$ & $\nu_{38}$ & 217 & $\mathrm{~b}_{2}$ & $10 \mathrm{~b}$ \\
\hline
\end{tabular}

Tabelle 4.4: Die 38 Normalschwingungen des Toluols, nach absteigender Energie sortiert [80]; Wnr.: Wilson-Nummer. Die Methylgruppe wird als freier Rotator angesehen. 
ist die Zahl der möglichen Fermi-Resonanzen stark eingeschränkt. So ist im Falle der nicht-totalsymmetrischen Normalmoden $\nu_{4-7}$ die Ausbildung dieser [1:2]-Resonanzen symmetrieverboten. Zudem sind Wechselwirkungen zwischen Methyl- und Arylstreckschwingungen ebenfalls zu vernachlässigen: Bei einem Vergleich der Spektren von Toluol mit Spektren partiell deuterierter Derivate wurden keine Hinweise auf derartige Kopplungen gefunden [81].

Diese Restriktionen beachtend, besitzen auch die Normalmoden $\nu_{1-3}$ nicht die Möglichkeit, Fermi-Resonanzen auszubilden. Die symmetrische Methylstreckschwingung $\nu_{8}$ dagegen wird intensiv an die isoenergetischen Obertöne der Moden $\nu_{13,14}$ koppeln.

Die Anzahl möglicher [1:(1+1)]-Resonanzen ist für die Moden $\nu_{1-8}$ ebenfalls sehr überschaubar. Von den nicht-totalsymmetrischen Moden $\nu_{4-7}$ sind derartige Kopplungen nur zwischen $\nu_{4}$ und $1 \nu_{9} 1 \nu_{15}$ bzw. $\nu_{5}$ und $1 \nu_{9} 1 \nu_{15}$ oder $1 \nu_{10} 1 \nu_{11}$. Die Normalmoden $\nu_{1-3}$ sind nahezu isoenergetisch mit dem Kombinationston $1 \nu_{9} 1 \nu_{11}$.

Experimentelle Nachweise konnten zudem für eine intensive Wechselwirkung innerhalb der Gruppe der $\mathrm{CH}_{3}$-Streckschwingungen sowie für eine Kopplung dieser Moden an Torsionsmoden des Toluols erbracht werden [82, 83].

\section{Helle Zustände im Experiment}

Das nIR-Absorptionsspektrum des Toluols im Wellenlängenbereich zwischen 1500 und $1800 \mathrm{~nm}$ ist in Abb. 4.7 abgebildet. Es wurde in verdünnter 1,1,2Trichlortrifluorethanlösung aufgenommen, kann aber trotzdem als Interpretationsgrundlage der Messungen in der Gasphase dienen, da die spektrale Verschiebung zwischen den Phasen weniger als $10 \mathrm{~cm}^{-1}$ beträgt und somit weitaus geringer als die spektrale Breite des Anregungspulses ausfällt. Die $6000 \mathrm{~cm}^{-1}$-Bande ist von weiteren nIR-Absorptionen, die Übergängen zu Kombinationstönen zuzuordnen sind, klar separiert. Sie besitzt ein intensives Maximum bei $1678 \mathrm{~nm}$. Dieses Maximum ist auf die Streckschwingungen ringgebundener Wasserstoffatome zurückzuführen, welche auch in höheren Obertönen stets eine intensive Absorbanz zeigen und gegenüber den Methylstreckschwingungen blauverschoben auftreten [81-85]. Der breite Untergrund der Bande enthält oberhalb von $1650 \mathrm{~nm}$ die Kombinationsmoden der Arylstreckschwingungen, zwischen $1715 \mathrm{~nm}$ und $1800 \mathrm{~nm}$ liegen die Obertöne der Methylstreckschwingungen vor [84]. Somit werden mit einem Anregungspuls der Wellenlänge $1678 \mathrm{~nm}$ bei einer Pulsbreite von ca. $30 \mathrm{~nm}$ vorwiegend Zustände mit zwei Quanten in den arylischen CH-Streckschwingungen 


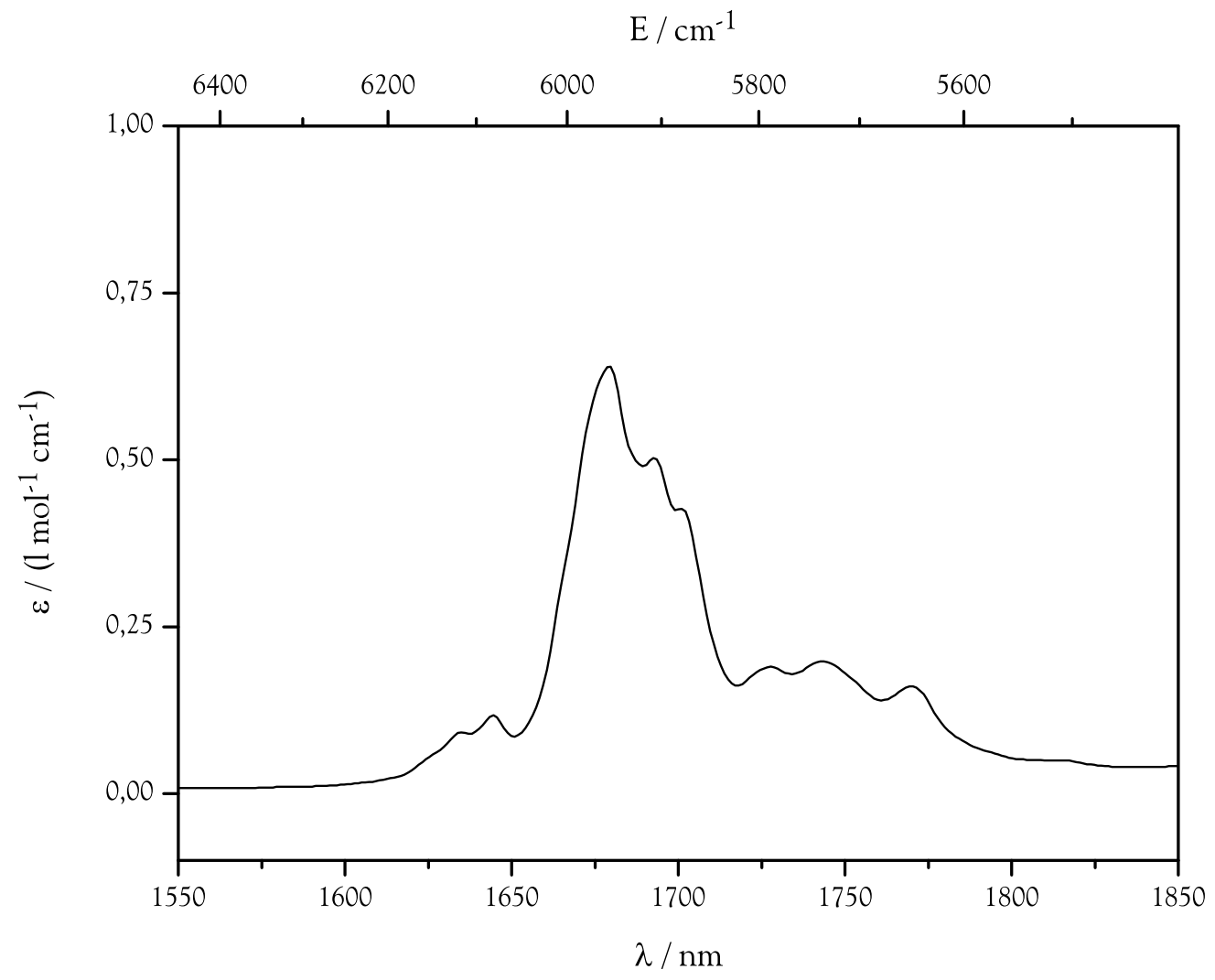

Abbildung 4.7: nIR-Absorptionsspektrum des Toluols

$\nu_{1-5}$ populiert. Eine partielle Lokalisierung der Anregungsenergie in methylischen $\mathrm{CH}-$ Streckschwingungen ist allerdings nicht völlig auszuschließen.

\section{FC-aktive Moden}

Der Bandenursprung des $\mathrm{S}_{1} \leftarrow \mathrm{S}_{0}$-Übergangs des Toluols liegt bei $37477 \mathrm{~cm}^{-1}$ $(267 \mathrm{~nm})$. Es handelt sich um einen $\mathrm{B}_{1} \leftarrow \mathrm{A}_{1}$-Übergang. Es liegt keine detaillierte Analyse der UV-Absorption auf der langwelligen Seite der (0,0)-Bande vor. Allerdings führte Bass eine ausführliche Fluoreszenzstudie durch, auf Grundlage derer die FC-aktiven Moden des Moleküls identifiziert werden können [86]. Dabei müssen zwei Annahmen vorausgesetzt werden: 
1. Die Minima der zweidimensionalen Potentialkurven aller FC-Moden liegen in $S_{1}$ und $S_{0}$ bei verschiedenen Gleichgewichtsabständen. Unter dieser Bedingung ist eine Änderung der Besetzung einzelner Moden beim Übergang wahrscheinlich, d.h. daß auf der roten Flanke des Bandenursprungs Absorptionsübergänge des Typs $\left(v^{\prime}=0, v^{\prime \prime}>0\right)$ auftreten. Da die Geometrie des $\mathrm{S}_{1}$-Toluols im Vergleich zum $\mathrm{S}_{0}$-Zustand insgesamt aufgeweitet ist, ist diese Annahme erfüllt.

2. Die Fluoreszenz erfolgt aus dem Schwingungsgrundzustand des $\mathrm{S}_{1}$. Somit kann die Wellenlänge der Fluoreszenzemission direkt mit der Wellenlänge der Absorption heißer Banden korreliert werden.

Im Experiment beträgt die Wellenlänge des UV-Probepulses $280 \mathrm{~nm}$. Im Fluoreszenzspektrum treten in diesem Bereich die Emissionslinien der sechs Moden $\nu_{18}, \nu_{19}, \nu_{25}, \nu_{33}, \nu_{34}$ und $\nu_{36}$ deutlich hervor. Die erhöhte Anzahl von FC-aktiven Moden im Vergleich zu den vorigen Modellsystemen ist wahrscheinlich auf die größere Komplexität des Moleküls zurückzuführen. Nichtsdestotrotz sind die Systeme so ähnlich, daß weiterhin die Schwingungen mit den Wilsonnummern 6 $\left(\nu_{33}\right)$ und $16\left(\nu_{36}\right)$ zum Franck-Condon-Übergang beitragen.

Eine verstärkte Absorption der heißen FC-Banden, wie sie in den Pump-ProbeExperimenten durch intramolekularen Energietransfer in die niederfrequenten Moden auftritt, kann auch durch eine vermehrte thermische Besetzung dieser Moden herbeigeführt werden. Dies wird zur Eichung der experimentellen Daten ausgenutzt. Abb. 4.8 enthält im oberen Teil nulllinienkorrigierte Absorptionsspektren, die von 50 bis $500^{\circ} \mathrm{C}$ in Schritten von ca. $25^{\circ} \mathrm{C}$ aufgezeichnet wurden. Das Maximum bei $267 \mathrm{~nm}$, welches dem (0,0)-Übergang zuzuschreiben ist, tritt in den Niedertemperaturspektren noch sehr deutlich auf, ebenso die benzolähnliche Progression zu kürzeren Wellenlängen hin. Mit steigender Temperatur findet eine zunehmende Verbreiterung des Spektrums sowie eine Rotverschiebung des globalen Absorptionsmaximums um $2 \mathrm{~nm}$ statt, die Schwingungsstruktur verschwindet schließlich ganz.

Den „heißen“ Spektren kann die Temperaturabhängigkeit der Absorptionskoeffizienten einzelner Wellenlängen entnommen und mit Gl. 4.1 in eine Energieabhängigkeit übersetzt werden. Dies führt zu den im unteren Teil von Abb. 4.8 dargestellten Eichkurven. Die Anpassungsparameter der für die Auswertung relevanten Kurve bei $280 \mathrm{~nm}$ sind in Anhang A.1 tabelliert. 

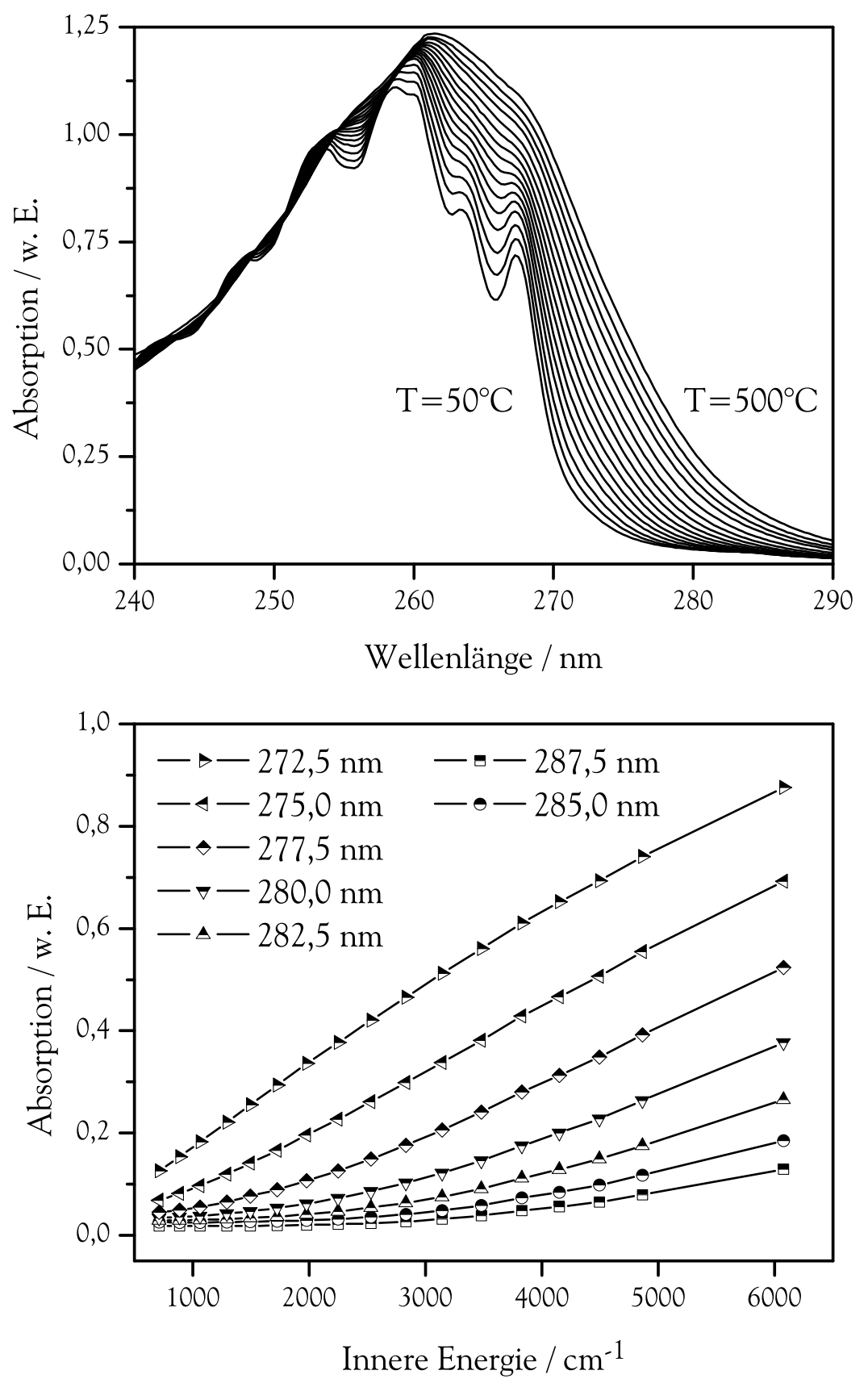

Abbildung 4.8: Temperaturabhängige UV-Absorptionsspektren des Toluols (oben) und daraus resultierende Energieabhängigkeit der Absorption (unten) 


\section{$4.5 \alpha, \alpha, \alpha$-Trifluortoluol}

In Analogie zum Toluol (vgl. Abschn. 4.4) gehört $\alpha, \alpha, \alpha$-Trifluortoluol $\left(\mathrm{C}_{6} \mathrm{H}_{5} \mathrm{CF}_{3}\right)$ ebenfalls zu den monosubstituierten $\mathrm{C}_{6} \mathrm{H}_{5} \mathrm{X}$-Benzolderivaten mit $C_{2 v}$-Symmetrie. Die $V_{6}$-Barriere des internen Rotators ist auch bei diesem Molekül mit $3.57 \mathrm{~cm}^{-1}$ so niedrig, daß von einer freien Rotation gesprochen werden kann [87]. Daher fehlt auch in diesem Fall die Torsionsschwingung der perfluorierten Methylgruppe in der Liste der Normalmoden des Moleküls, die in Tab. 4.5 mitsamt ihrer Symmetrie und - wo möglich - Wilsonnummer aufgeführt sind. Die Fundamentalen können wie beim Toluol in 30 Phenylschwingungen der irreduziblen Darstellungen $11 a_{1}+$ $3 a_{2}+10 b_{1}+6 b_{2}$ und acht Fragmentschwingungen mit $2 a_{1}+3 b_{2}+3 b_{3}$ unterschieden werden.

$\mathrm{Zu}$ letzteren zählen drei CF-Streckschwingungen $\left(\nu_{10}, \nu_{14}, \nu_{16}\right)$, drei Knickschwingungen $\left(\nu_{25}, \nu_{29}, \nu_{31}\right)$ und zwei Pendelschwingungen $\left(\nu_{34}, \nu_{36}\right)$.

Der Vergleich der Arylschwingungsfrequenzen des $\alpha, \alpha, \alpha$-Trifluortoluols mit denen der übrigen Modellsysteme anhand des Wilsonsystems gestaltet sich schwierig, da die Zuordnung der Wilsonnummern einiger Schwingungen in der Literatur nicht einstimmig ist $[60,88]$. Dies betrifft hauptsächlich Moden mit Frequenzen oberhalb von $1000 \mathrm{~cm}^{-1}$, daher ist die vergleichende Diskussion der FC-aktiven Moden aller Modellsysteme weiterhin zulässig.

Die Fluorierung des Methylrotators vergrößert die Anzahl niederfrequenter Moden des Moleküls im Vergleich zu Toluol signifikant. Die Auswirkung auf die Zustandsdichte dieses Modellsystems wird in Abschnitt 4.6 erläutert. Dennoch ist $\mathrm{zu}$ erwarten, daß auch im $\mathrm{C}_{6} \mathrm{H}_{5} \mathrm{CF}_{3}$ die nahezu isoenergetischen CH-Streckschwingungen $\nu_{1-5}$ und Kombinationstöne der Moden $\nu_{6-9}$ in Resonanz miteinander stehen, da deren Frequenzen vom Substituenten weitesgehend unbeeinträchtigt sind.

\section{Helle Zustände im Experiment}

Mit der Perfluorierung der Methylgruppe entfallen im nIR-Spektrum zwischen 1500 und $1800 \mathrm{~cm}^{-1}$ diejenigen Absorptionsbanden, die beim Toluol den Ober- bzw. Kombinationstönen der alkylischen CH-Streckschwingungen zugeschrieben werden konnten. Bei einem Vergleich des Absorptionsspektrums des $\alpha, \alpha, \alpha$-Trifluortoluols(Abb. 4.9) mit dem des Toluols (Abb. 4.7) fällt besonders die Abwesenheit der breiten Schulter um $5800 \mathrm{~cm}^{-1}$ auf. Darüber hinaus ist die 
gesamte spektrale Signatur schmaler und homogener. Das Maximum der Absorption liegt bei $1664 \mathrm{~nm}\left(6010 \mathrm{~cm}^{-1}\right)$. Analog zu den anderen drei Modellsystemen mit $\mathrm{C}_{2 v}$-Symmetrie ist diese Bande in erster Linie den Obertönen $2 \nu_{1-5}$ der phenylischen CH-Streckschwingungen zuzuordnen. Der schwache Untergrund der Bande enthält desweiteren Kombinationstöne der CH-Streckschwingungen untereinander $\left(>6100 \mathrm{~cm}^{-1}\right)$ und mit CC-Gerüstschwingungen $\left(<5900 \mathrm{~cm}^{-1}\right)$. Die Wellenlänge des IR-Anregungspulses in den Pump-Probe-Experimenten an $\alpha, \alpha, \alpha$-Trifluortoluol wird auf das Maximum der $2 v_{\mathrm{CH}}$-Bande bei $1664 \mathrm{~nm}$ eingestellt.

\begin{tabular}{|cccc|cccc|}
\hline Mode & $\begin{array}{c}\text { Energie } \\
{\left[\mathbf{c m}^{-1}\right]}\end{array}$ & Sym. & Wnr. & Mode & $\begin{array}{c}\text { Energie } \\
{\left[\mathbf{c m}^{-\mathbf{1}}\right]}\end{array}$ & Sym. & Wnr. \\
\hline \hline$\nu_{1}$ & 3096 & $\mathrm{a}_{1}$ & $20 \mathrm{a}$ & $\nu_{20}$ & 1004 & $\mathrm{a}_{1}$ & 1 \\
$\nu_{2}$ & 3096 & $\mathrm{~b}_{1}$ & $20 \mathrm{~b}$ & $\nu_{21}$ & 990 & $\mathrm{~b}_{2}$ & 5 \\
$\nu_{3}$ & 3076 & $\mathrm{a}_{1}$ & 2 & $\nu_{22}$ & 970 & $\mathrm{a}_{2}$ & $17 \mathrm{a}$ \\
$\nu_{4}$ & 3076 & $\mathrm{~b}_{1}$ & $7 \mathrm{~b}$ & $\nu_{23}$ & 923 & $\mathrm{~b}_{2}$ & $17 \mathrm{~b}$ \\
$\nu_{5}$ & 3022 & $\mathrm{a}_{1}$ & 13 & $\nu_{24}$ & 844 & $\mathrm{a}_{2}$ & $10 \mathrm{a}$ \\
$\nu_{6}$ & 1614 & $\mathrm{~b}_{1}$ & $8 \mathrm{~b}$ & $\nu_{25}$ & 770 & $\mathrm{a}_{1}$ & - \\
$\nu_{7}$ & 1593 & $\mathrm{a}_{1}$ & $8 \mathrm{a}$ & $\nu_{26}$ & 770 & $\mathrm{~b}_{2}$ & $10 \mathrm{~b}$ \\
$\nu_{8}$ & 1459 & $\mathrm{a}_{1}$ & $19 \mathrm{a}$ & $\nu_{27}$ & 695 & $\mathrm{~b}_{2}$ & 4 \\
$\nu_{9}$ & 1459 & $\mathrm{~b}_{1}$ & $19 \mathrm{~b}$ & $\nu_{28}$ & 657 & $\mathrm{a}_{1}$ & 12 \\
$\nu_{10}$ & 1328 & $\mathrm{a}_{1}$ & - & $\nu_{29}$ & 657 & $\mathrm{~b}_{1}$ & - \\
$\nu_{11}$ & 1328 & $\mathrm{~b}_{1}$ & 14 & $\nu_{30}$ & 618 & $\mathrm{~b}_{1}$ & $6 \mathrm{~b}$ \\
$\nu_{12}$ & 1253 & $\mathrm{~b}_{1}$ & 3 & $\nu_{31}$ & 596 & $\mathrm{~b}_{2}$ & - \\
$\nu_{13}$ & 1187 & $\mathrm{a}_{1}$ & $9 \mathrm{a}$ & $\nu_{32}$ & 485 & $\mathrm{~b}_{2}$ & $16 \mathrm{~b}$ \\
$\nu_{14}$ & 1179 & $\mathrm{~b}_{1}$ & - & $\nu_{33}$ & 398 & $\mathrm{a}_{2}$ & $16 \mathrm{a}$ \\
$\nu_{15}$ & 1161 & $\mathrm{~b}_{1}$ & $9 \mathrm{~b}$ & $\nu_{34}$ & 398 & $\mathrm{~b}_{1}$ & - \\
$\nu_{16}$ & 1152 & $\mathrm{~b}_{2}$ & - & $\nu_{35}$ & 336 & $\mathrm{a}_{1}$ & $6 \mathrm{a}$ \\
$\nu_{17}$ & 1072 & $\mathrm{a}_{1}$ & $7 \mathrm{a}$ & $\nu_{36}$ & 317 & $\mathrm{~b}_{2}$ & - \\
$\nu_{18}$ & 1072 & $\mathrm{~b}_{1}$ & 15 & $\nu_{37}$ & 169 & $\mathrm{~b}_{1}$ & $18 \mathrm{~b}$ \\
$\nu_{19}$ & 1028 & $\mathrm{a}_{1}$ & $18 \mathrm{a}$ & $\nu_{38}$ & 139 & $\mathrm{~b}_{2}$ & 11 \\
\hline
\end{tabular}

Tabelle 4.5: Die 38 Normalschwingungen des $\alpha, \alpha, \alpha$-Trifluortoluols, nach absteigender Energie sortiert [88]; Wnr.: Wilson-Nummer. Die Methylgruppe wird als freier Rotator angesehen. 


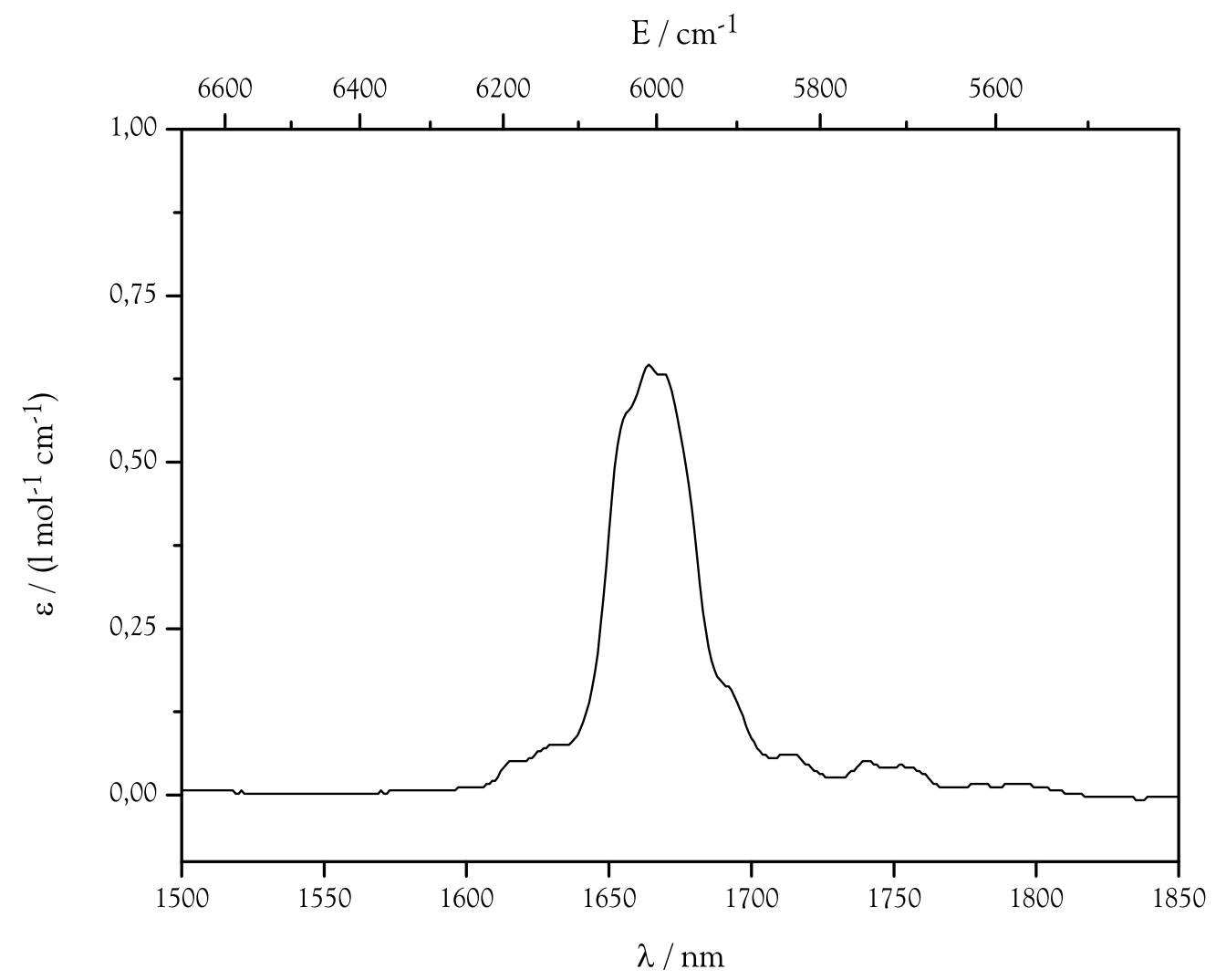

Abbildung 4.9: nIR-Absorptionsspektrum des $\alpha, \alpha, \alpha$-Trifluortoluols

\section{FC-aktive Moden}

Der erste elektronische Übergang des $\alpha, \alpha, \alpha$-Trifluortoluols ist vom Typ $\mathrm{B}_{1} \leftarrow \mathrm{A}_{1}$ und hat seinen Ursprung bei $37819 \mathrm{~cm}^{-1}(264 \mathrm{~nm})$. Mit Pulsen, deren Energie kleiner als die (0,0)-Differenz ist, werden $\mathrm{S}_{1} \leftarrow \mathrm{S}_{0}$-Übergänge aus schwingungsangeregten Zuständen des Moleküls realisiert. Die zugrundeliegenden FC-aktiven Moden konnten für $\alpha, \alpha, \alpha$-Trifluortoluolanhand kombinierter Absorptions- und Fluoreszenzstudien eindeutig identifiziert werden, da dieses Molekül die auf S. 82 geforderten Bedingungen nachweislich erfüllt [89]. Es handelt sich um die Moden $\nu_{20}, \nu_{26}, \nu_{28}, \nu_{30}, \nu_{35}$ und $\nu_{38}$. Höherfrequente Normalmoden des Modellsystems zeigen keine UV-Aktivität. Eine intensive Fluoreszenzemission bei der Wellenlän- 
ge des Probepulses $(275 \mathrm{~nm})$ rührt von einer Kombinationsanregung der Moden $\nu_{20}$ (Wilsonnr. 1), $\nu_{35}$ (6a) und $\nu_{38}$ (11) her. Somit tragen wie bei allen anderen in dieser Arbeit untersuchten Aromaten die beiden aus der entarteten Wilsonmode 6 des Benzols entstandenen Schwingungen 6a und 6b signifikant zum Pump-ProbeSignal bei.

Die Auswirkung einer thermischen Anregung des Moleküls auf sein UVAbsorptionsspektrum ist in der oberen Graphik der Abb. 4.10 veranschaulicht. Gezeigt sind Gasphasenspektren, die bei Temperaturen von 50 bis $500{ }^{\circ} \mathrm{C}$ in $25^{\circ} \mathrm{C}$ Schritten aufgezeichnet wurden. Bei niedrigen Temperaturen ist eine klare Progression auf der blauen Flanke der (0,0)-Bande erkennbar, sowie das Absorptionsmaximum der heißen Banden auf der roten Seite. Diese Strukturierung verschwindet mit steigender Temperatur zunehmend. Die Intensität des Übergangs nimmt zu höheren Temperaturen über die gesamte Breite des Spektrums zu, ein isospestischer Punkt ist nicht zu erkennen.

Schnitte durch die Temperaturprofile zeigen die Temperaturabhängigkeit der wellenlängenspezifischen Absorptionskoeffizienten, die mittels Gl. 4.1 in die Abhängigkeit von der inneren Energie umgerechnet werden kann. Die energieabhängigen optischen Dichten ausgewählter Wellenlängen, darunter der Probewellenlänge, sind in der unteren Hälfte von Abb. 4.10 dargestellt. Diese können mittels eines Polynoms 3. Grades angepaßst und als Eichkurven in die Auswertung der transienten Absorptionen (s. Kap. 6) einfließen. Die Parameter der Polynomanpassung sind in Anhang A.1 aufgelistet. 

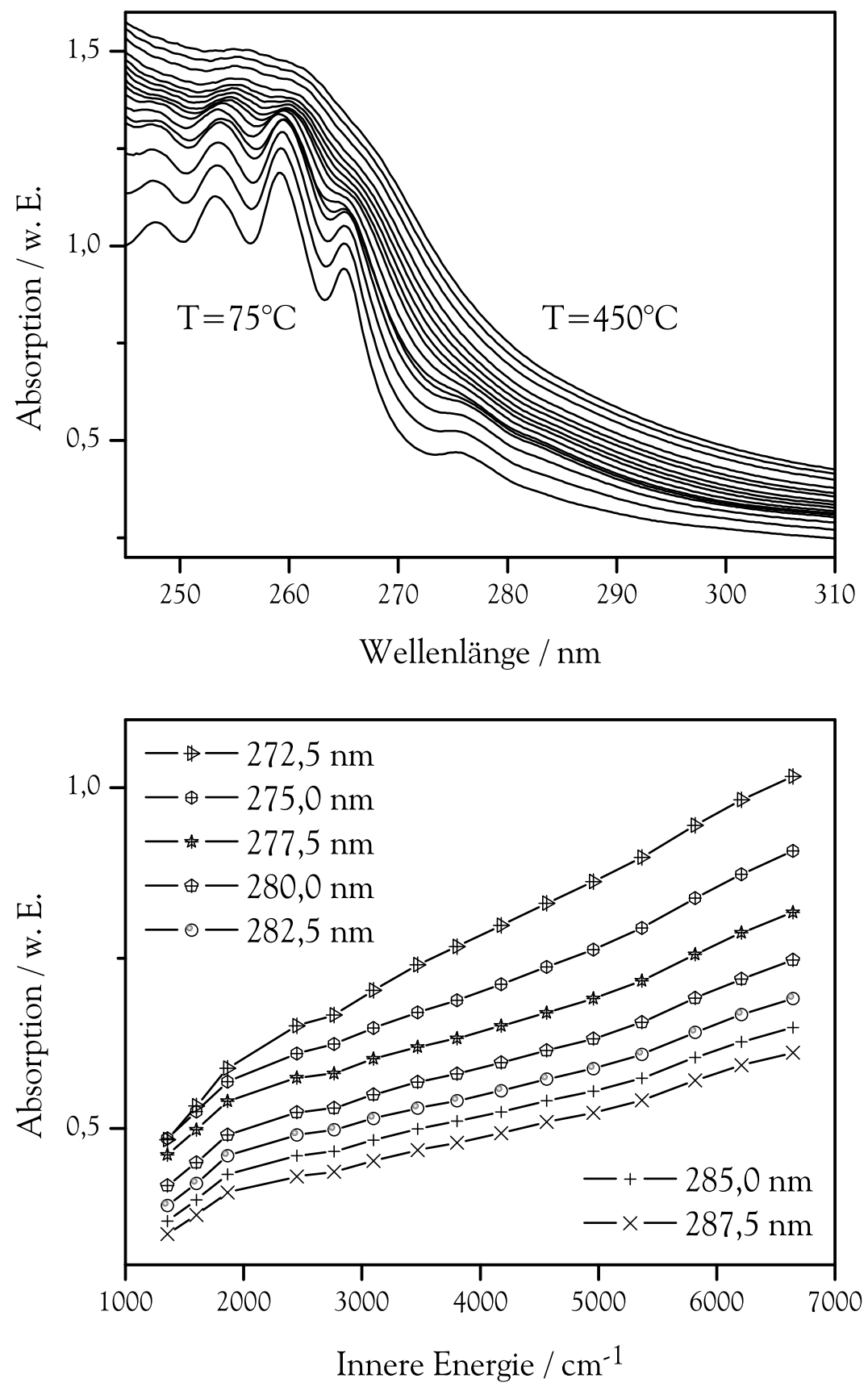

Abbildung 4.10: Temperaturabhängige UV-Absorptionsspektren des $\alpha, \alpha, \alpha$-Trifluortoluols(oben) und daraus resultierende Energieabhängigkeit der Absorption (unten) 


\subsection{Zustandsdichten}

In diesem Abschnitt sollen abschließend die fünf aromatischen Modellsysteme in Hinblick auf ihre Energieniveaustruktur der Fundamentalschwingungen und die daraus resultierende Zustandsdichte verglichen werden.

Die Frequenzen der Normalmoden jedes Modellsystems sind in Abb. 4.11 als Energieniveaudiagramm dargestellt. Entartete Schwingungen sind aus Gründen der Übersichtlichkeit nur für Benzol besonders gekennzeichnet. Darüber hinaus sind die jeweils acht Substituentenmoden der Moleküle Toluol und $\alpha, \alpha, \alpha$-Trifluortoluol mit gestrichelten Niveaulinien hervorgehoben. Sie lassen sich nicht nach dem Wilsonsystem für arylische Schwingungen klassifizieren und stellen quasi ein den phenylischen Schwingungen überlagertes Modensystem dar.

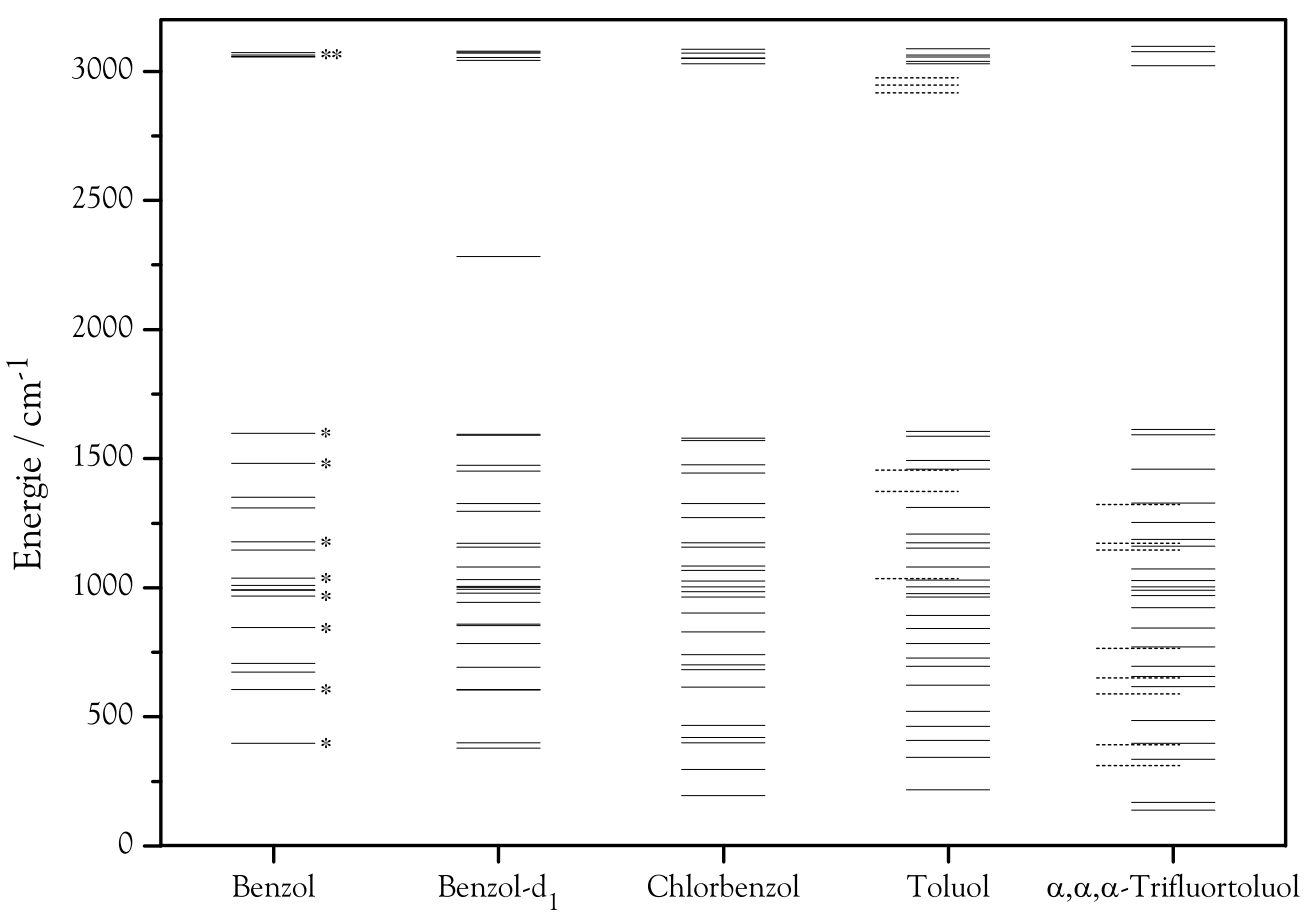

Abbildung 4.11: Energieniveau-Diagramm der Fundamentalschwingungen der fünf Modellsysteme (Stern: entartet; gestrichelt: nicht nach Wilson klassifizierbare Substituentenschwingungen) 


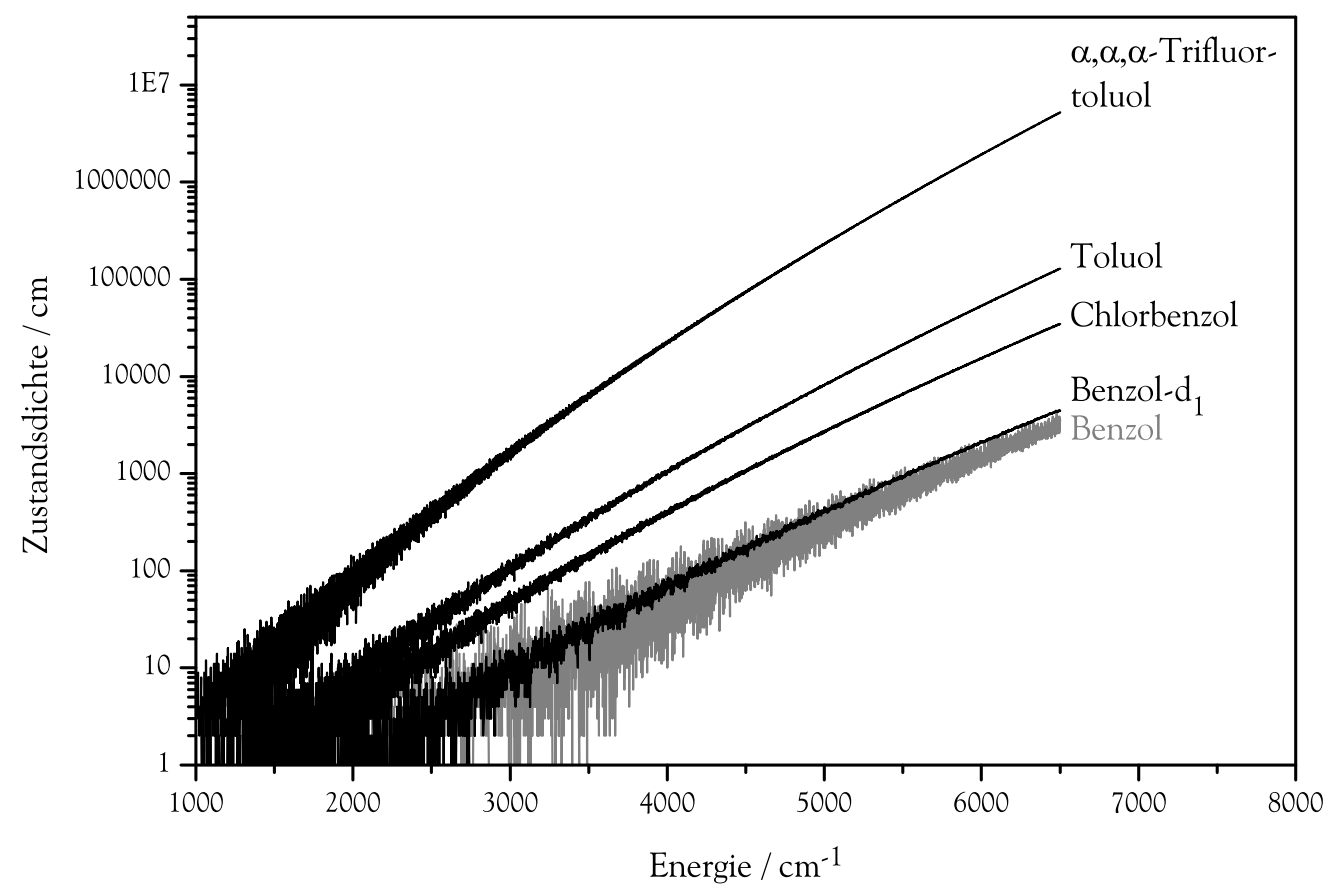

Abbildung 4.12: Vergleich der Zustandsdichten der fünf Modellsysteme

Die benzoltypische Niveaustruktur mit energetisch stark abgesetzten $\mathrm{CH}-$ Streckschwingungen bleibt auch in den substituierten Derivaten weitesgehend erhalten. Dagegen ist deutlich eine Zunahme von niederfrequenten Normalmoden $\left(\nu \leq 1000 \mathrm{~cm}^{-1}\right)$ in der Reihenfolge Benzol, Benzol- $\mathrm{d}_{1}$, Chlorbenzol, Toluol, $\alpha, \alpha, \alpha$-Trifluortoluol zu erkennen. Hierfür ist zum einen die mit der Symmetriereduktion von $\mathrm{D}_{6 \mathrm{~h}}$ auf $\mathrm{C}_{2 \mathrm{v}}$ einhergehende Aufhebung der Entartung von zehn Benzolnormalmoden verantwortlich sowie bei Toluol und $\alpha, \alpha, \alpha$-Trifluortoluol die Zunahme der Gesamtzahl von Oszillatoren. Zum anderen sind Kraftkonstanten der CX-Bindungen (X = Subst.) kleiner als die der CH-Bindung, wodurch Schwingungen mit großem Substituentenanteil energetisch abgesenkt werden.

Die Anzahl niederfrequenter Normalmoden ist der die Molekülzustandsdichte am stärksten beeinflussende Faktor. Abb. 4.12 zeigt die Zustandsdichten der fünf Aromaten für Innere Energien bis $6500 \mathrm{~cm}^{-1}$, was die im Experiment präparierten hellen Zustände mit einschließt. Für die Moleküle Benzol, Benzol- $\mathrm{d}_{1}$ und Chlorbenzol handelt es sich um reine Schwingungszustandsdichten, deren Berechnung 
Kapitel 4 Modellsysteme

mittels des Beyer-Swinehardt-Zählalgorithmus [90,91] auf der Basis der Normalmodenfrequenzen erfolgte. Im Falle des Toluols und $\alpha, \alpha, \alpha$-Trifluortoluols wurde dieser Algorithmus modifiziert, um die Rotationszustände der Methylgruppe zu inkorporieren [92]. Details der Rechnung sind in Anhang A.2 aufgeführt.

Die augenscheinlich starken Oszillationen der Zustandsdichten bei unter $3000 \mathrm{~cm}^{-1}$ sind im Falle der Derivate (schwarze Kurven) auf die semilogarithmische Auftragung zurückzuführen. Benzol (grau) stellt dagegen einen Sonderfall dar: Hier weist die Zustandsdichte auch bei Energien, die den $\left(v_{\mathrm{CH}}=\right.$ 2)-Zuständen des Experiments entsprechen, starke Modulationen auf. Die Ursache hierfür liegt in der oben erwähnten Entartung eines Drittels der Normalmoden dieses Moleküls und darin, daß es keine sub- $400 \mathrm{~cm}^{-1}$-Schwingungen besitzt.

\section{Zustandsdichte und QNS}

Aus der Zustandsdichte eines Moleküls lassen sich einige Rückschlüsse auf die Natur seines quantum number space (QNS, siehe Abschn. 2.1.3) ziehen. So ist bei einer Zustandsdichte, die wie bei Benzol- $\mathrm{d}_{1}$, Chlorbenzol, Toluol und $\alpha, \alpha, \alpha$-Trifluortoluol monoton und unmoduliert mit der Energie ansteigt, eine isotrope Verteilung der Zustandsgitterpunkte innerhalb einer Energieschale des QNS zu erwarten. Je größer die Zustandsdichte, desto mehr Gitterpunkte liegen in einer Schale.

Es ist anzunehmen, daß sich der QNS des Benzols strukturell von denen der anderen vier Aromaten signifikant unterscheidet. Die starke Modulation der $\mathrm{Zu}-$ standsdichte läßt eine anisotrope Verteilung der Zustandspunkte im QNS vermuten. Eine Anordnung aller Zustände zu Quasiclustern mit Energiebreiten, die den lokalen Maxima und Minima der Zustandsdichte zu entnehmen sind, ist wahrscheinlich. Darüber hinaus werden aufgrund der hohen Anzahl entarteter Normalmoden entsprechend viele entartete Zustände auftreten, deren QNS-Punkte jeweils identisch sind. Daher beträgt die Gitterpunktdichte pro Energieschale beim Benzol vermutlich weit weniger als bei den übrigen Modellsystemen. 


\section{Kapitel 5}

\section{Ergebnisse}

Um die Mechanismen des intramolekularen Energietransfers von aromatischen Molekülen zu aufzuklären, fand im Rahmen dieser Arbeit eine kombinierte experimentelle und theoretische Studie des Phänomens statt. Den Ergebnissen dieser Untersuchungen ist das folgende Kapitel gewidmet. In den ersten beiden Abschnitten sind die Ergebnisse der zeitaufgelösten IR-Pump-UV-ProbeExperimente zusammengefaßt. Daran schließen sich die Resultate der Simulationsrechnungen auf Basis des IVR-Diffusionsmodells an.

Es werden zuerst die Gasphasenmessungen an den reinen Modellsystemen Benzol, Benzol- $\mathrm{d}_{1}$, Chlorbenzol, Toluol und $\alpha, \alpha, \alpha$-Trifluortoluol präsentiert. An gasförmigem Benzol wurden sowohl temperatur- als auch druckabhängige Messungen vorgenommen. Darüber hinaus wurde bei den beiden Modellsystemen Benzol und Benzol- $\mathrm{d}_{1}$ die Anregungswellenlänge variiert und somit die Relaxation unterschiedlicher heller Zustände nullter Ordnung studiert. Diese Daten ermöglichen es, den Einfluß der chemischen Konstitution und molekularen Symmetrie auf den Schwingungsenergietransfer zu bestimmen.

Der zweite Teil des Kapitels stellt die Ergebnisse der Experimente in Anwesenheit gasförmiger und überkritischer Stoßpartner vor. Die hierfür verwendeten Inertgase sind Helium, Argon, Kohlendioxid, 1,1,2-Trichlortrifluorethan und $n$ Perfluorpentan. Die Durchführung dieser Experimente gestaltete sich durchweg sehr schwierig; in einigen Fällen konnten dennoch Messungen bei verschiedenen Stoßgaskonzentrationen durchgeführt werden. Aus diesen Daten können Erkenntnisse über die Rolle der molekularen Umgebung bei der intramolekularen Relaxation gewonnen werden.

Die Observable der Experimente ist die zeitliche Änderung der optischen Dichte, $\triangle \mathrm{OD}$, bei der Wellenlänge des ultravioletten Probepulses. Um einen Vergleich 
Kapitel 5 Ergebnisse

der Daten zu erleichtern, wurden sämtliche transiente Absorptionsspuren auf ihr Signalmaximum $\Delta \mathrm{OD}_{\max }$ normiert. Die maximale Verzögerungszeit beträgt in den meisten Messungen nicht mehr als 250 ps, da für längere Zeiten ein optimal kollinearer Überlapp zwischen Pump- und Probestrahl (s. Abschn. 3.2.4) nicht mehr gewährleistet werden konnte. In allen transienten Signalen ist die „echte“ Dynamik im Zeitnullpunkt mit einem Absorptionsartefakt der IR-UVKreuzkorrelation überlagert, welches generell mind. eine Größenordnung intensiver als das maximale Signal erscheint und die Zeitauflösung der Messung begrenzt (vgl. Abschn. 3.2.4). Der Übersichtlichkeit halber wird dieses Artefakt in den folgenden Ergebnisdarstellungen nicht gezeigt.

\subsection{Gasphasenexperimente an quasi-isolierten Molekülen}

\subsubsection{Messungen an Benzol}

Die Messungen an reinem gasförmigem Benzol dienen mehreren Zwecken. Primär geben sie zunächst Auskunft über den Schwingungsenergietransfer in diesem Molekül. Darüber hinaus bilden sie aber auch die Referenz für alle weiteren Untersuchungen dieser Arbeit, sowohl in Hinblick auf den Einfluß chemischer Substitution, die einen Eingriff am Referenzmolekül selber darstellt, als auch in Bezug auf die Experimente mit zusätzlichen Stoßpartnern, bei denen die molekulare Umgebung des Referenzsystems geändert wird.

\section{Energieabhängige Messungen}

An Benzol wurden Experimente unter Variation der Meßstemperatur, des Druckes, der Pumpwellenlänge und des Badgases durchgeführt. Die Referenz all dieser Versuche stellt das in Abb. 5.1 (bis 120 ps) und im obersten Graphen der Abb. 5.2 (bis $400 \mathrm{ps)} \mathrm{gezeigte} \mathrm{transiente} \mathrm{Absorptionsprofil} \mathrm{dar.} \mathrm{Es} \mathrm{wurde} \mathrm{bei} \mathrm{einer} \mathrm{Tem-}$ peratur von $100^{\circ} \mathrm{C}$ und einem Benzoldruck von 0.5 bar unter Verwendung eines Pumppulses mit $1670 \mathrm{~nm}$ und eines Probepulses mit $270 \mathrm{~nm}$ Wellenlänge aufgenommen. Der so präparierte ZOBS (zeroth order bright state, s. S. 10) kann als $2 v_{\mathrm{CH}}$-Kombinationston von CH-Streckschwingungen mit je ca. $3000 \mathrm{~cm}^{-1}$ aufge- 
5.1 Gasphasenexperimente an quasi-isolierten Molekülen

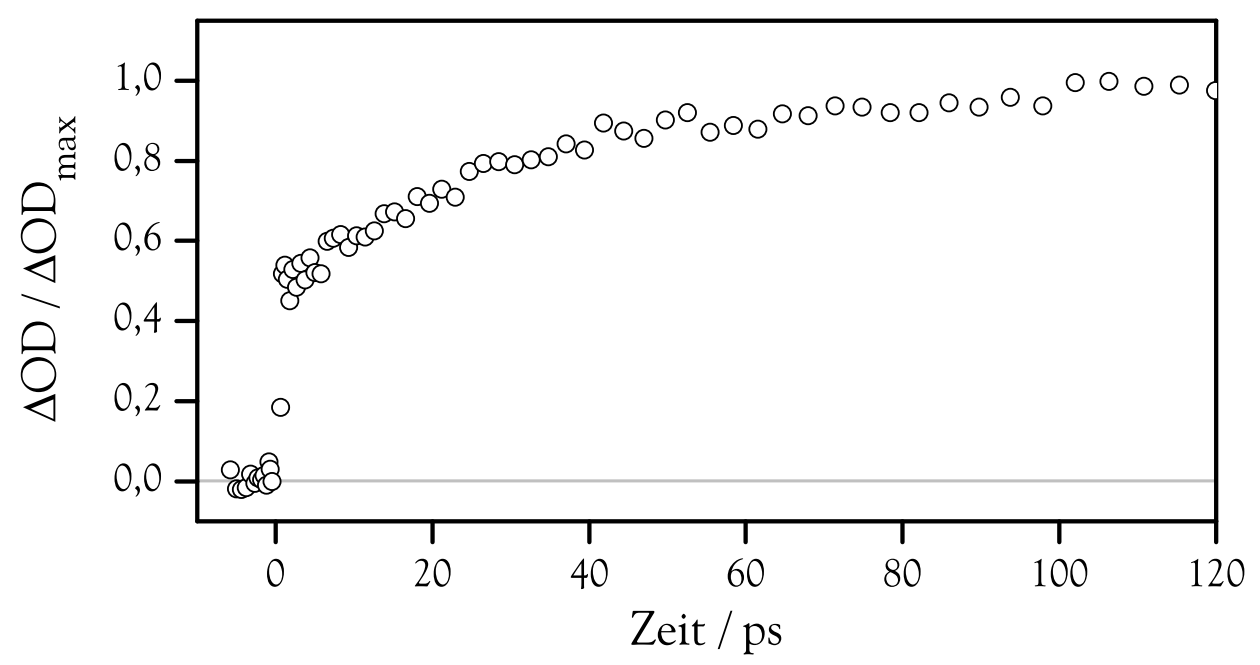

Abbildung 5.1: Hochaufgelöste transiente Absorption des reinen gasförmigen Benzols $\left(\mathrm{T}=100^{\circ} \mathrm{C} ; \mathrm{p}=0.5 \mathrm{bar}, \lambda_{\text {Pump }}=1670 \mathrm{~nm}, \lambda_{\text {Probe }}=270 \mathrm{~nm}\right)$

faßst werden (vgl. Abschn. 4.1). Die Abfrage der Dynamik erfolgte über die heißen Banden der niederfrequentesten Normalmoden des Moleküls.

Das oberste Pump-Probe-Signal in Abb. 5.2 weist einige Merkmale auf, die sich als charakteristisch für alle Gasphasenmessungen an aromatischen Modellsystemen erweisen werden. Am augenfälligsten ist das Fehlen eines Signalrückgangs auf die Grundlinie (grau), wie sie der VET-Dynamik zuzuschreiben wäre und im Beispielsignal (Abb. 3.2 auf S. 30) gezeigt ist. Die Begründung hierfür liegt in der erwünschten starken Reduktion der VET-Geschwindigkeit in der Gasphase durch die verringerte intermolekulare Stoßfrequenz. Mögliche zusätzliche Effekte von Selbststößen auf die UV-Absorption werden in Abschn. 6.1.1 diskutiert.

Der Anstieg des Signals - und damit die Dynamik der intramolekuleren Energieumverteilung - setzt sich offensichtlich aus zwei Komponenten zusammen. Innerhalb der ersten Pikosekunde erfolgt ein innerhalb der Zeitauflösung nicht vollständig aufzulösender Sprung der Intensität auf ca. 0.5 $\Delta \mathrm{OD}_{\max }$. Daran schließst zweiter Anstieg auf einer langsameren Zeitskala an, der nach ca. 100 ps endet. Die Form des Signalwachstums ist definitiv nicht-monoexponentiell; die Existenz von mehr als zwei IVR-Zeitskalen kann anhand der vorliegenden Messun- 

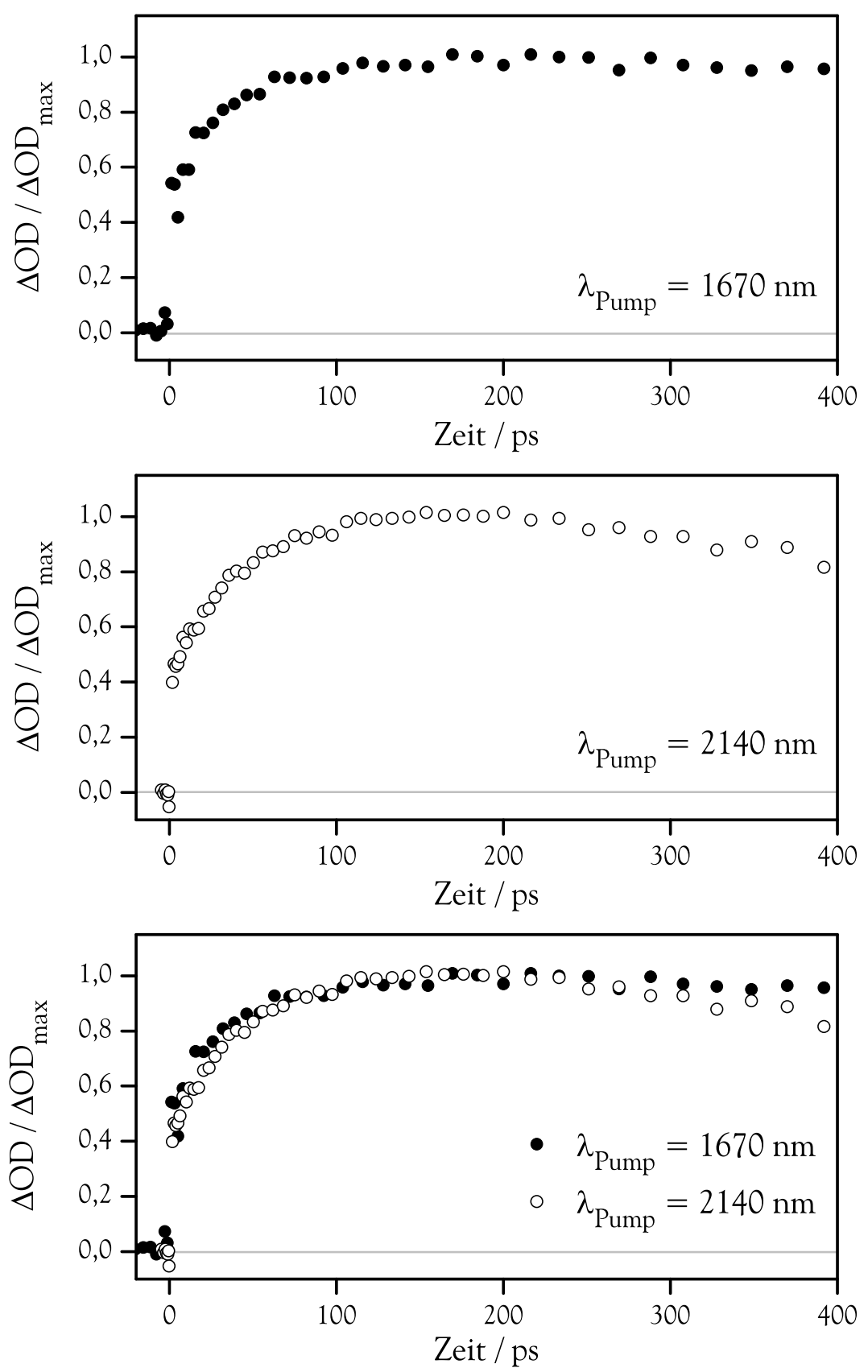

Abbildung 5.2: Transiente Absorption des reinen gasförmigen Benzols bei verschiedenen Anregungsenergien $\left(\mathrm{T}=100^{\circ} \mathrm{C} ; \mathrm{p}=0.5 \mathrm{bar}, \lambda_{\text {Probe }}=270 \mathrm{~nm}\right)$ 
5.1 Gasphasenexperimente an quasi-isolierten Molekülen

gen nicht ausgeschlossen werden. Eine genauere Analyse der funktionalen Form der Absorptions-Zeit-Profile erfolgt im Rahmen der kinetischen Modellierung der Pump-Probe-Experimente in Kap. 6.

Die mittlere Darstellung in Abb. 5.2 zeigt das transiente Absorptionsprofil nach Anregung mit infrarotem Licht der Wellenlänge $2140 \mathrm{~nm}$. In diesem Fall ist der ZOBS als Kombinationston von CH-Streck- mit CH-Biegeschwingungen (vgl. Abschn. 4.1) aufzufassen. Das Signal zeigt ebenfalls einen spontanen Anstieg innerhalb der Zeitauflösung auf ca. $0.45 \Delta \mathrm{OD}_{\max }$, dem sich eine zweite, langsamer ansteigende Komponente anschließst, bis nach ca. 100 ps das Maximum erreicht ist. Es folgt nach ca. 200 ps ein allmählicher Rückgang der Signalintensität auf $0.8 \Delta \mathrm{OD}_{\max }$ nach $400 \mathrm{ps}$.

In beiden Fällen konnte dank kollinearer Strahlführung und vielfacher Mittelungen trotz des geringen Absorptionsquerschnitts der Proben ein ausgezeichnetes Signal-zu-Rausch-Verhältnis erreicht werden.

Ein Vergleich der beiden Absorptions-Zeit-Profile mit unterschiedlicher Anregungsenergie ist im untersten Graphen der Abb. 5.2 ermöglicht. Die Natur des ZOBS hat nur eine Auswirkung auf die intramolekulare Dynamik der Energieumverteilung. Die sub-ps-Komponente des Prozesses bleibt unbeeinflußt, während die zweite, langsame Dynamik im Fall der niederenergetischeren Anregung mit $4500 \mathrm{~cm}^{-1}$ im Vergleich zu jener mit $6000 \mathrm{~cm}^{-1}$ bis $100 \mathrm{ps}$ etwas langsamer erscheint. Im Gegensatz hierzu fällt zu langen Zeiten das Signal bei $2140 \mathrm{~nm}$ Pumpwellenlänge etwas schneller als das bei $1670 \mathrm{~nm}$ ab.

\section{Temperaturabhängige Messungen}

Die hier vorgestellten Pump-Probe-Experimente an gasförmigen Substanzen stellen derzeit die Grenze des Möglichen im Bereich zeitabhängiger Absorptionsmethoden dar. Extrem niedrige Konzentrationen und geringe Absorptionsquerschnitte für Obertonanregungen erfordern einen Substanzdruck von mindestens 0.5 bar, um eine vertretbare Signalintensität zu erhalten. Je nach Siedepunkt der jeweiligen Spezies mußte daher die Meßstemperatur so gewählt werden, daß ein entsprechend hoher Dampfdruck gewährleistet war. Es ergaben sich für die fünf im Rahmen dieser Arbeit untersuchten Modellsysteme Meßtemperaturen zwischen 100 und $140^{\circ} \mathrm{C}$. Ein Vergleich der transienten Absorptionen kann allerdings nur erfolgen, wenn eine etwaige Temperaturabhängigkeit der Energietransferdynamik ausgeschlossen oder zumindest quantifiziert werden kann. Aus die- 

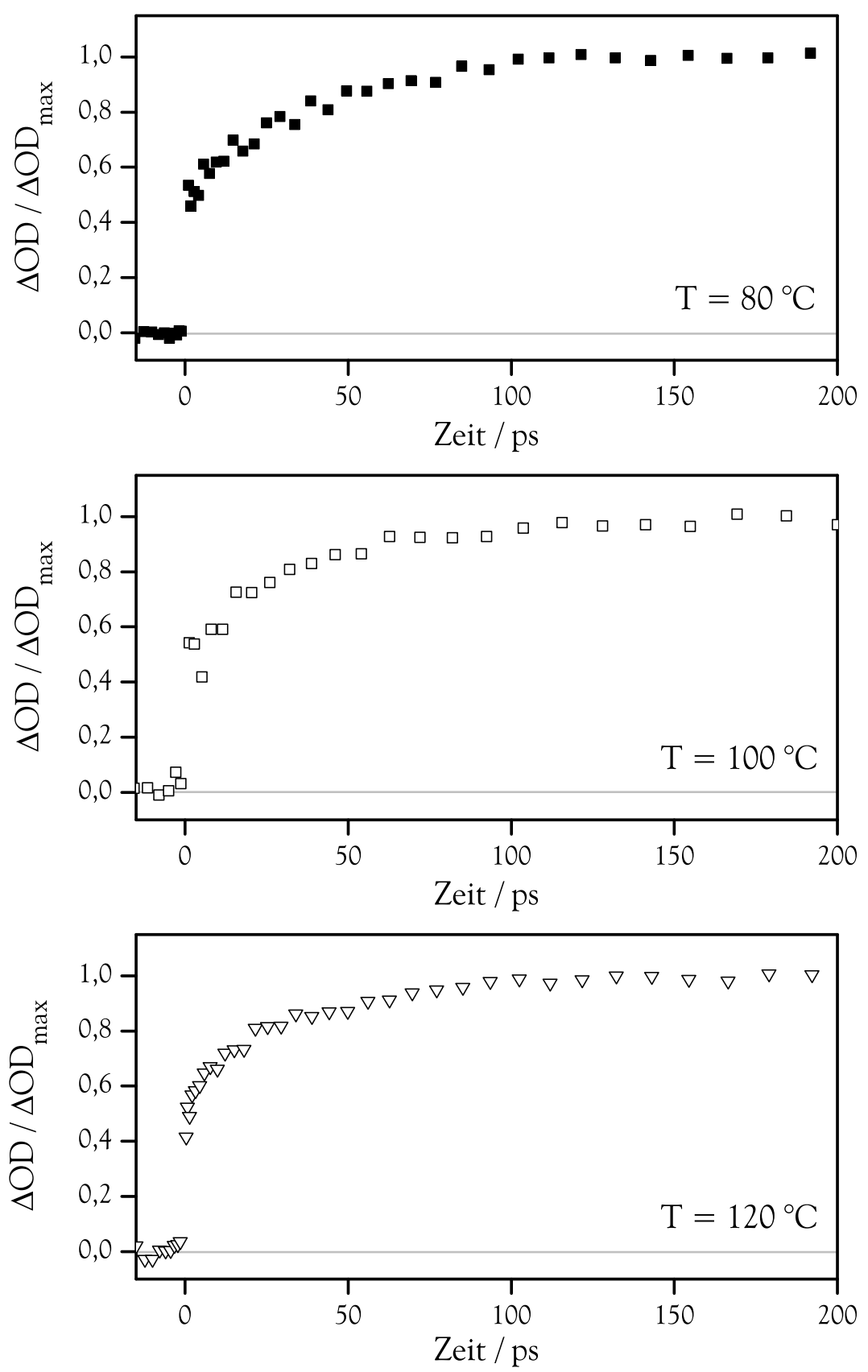

Abbildung 5.3: Transiente Absorption des reinen gasförmigen Benzols bei verschiedenen Temperaturen I $\left(\mathrm{p}=0.5 \mathrm{bar}, \lambda_{\text {Pump }}=1670 \mathrm{~nm}, \lambda_{\text {Probe }}=270 \mathrm{~nm}\right)$ 

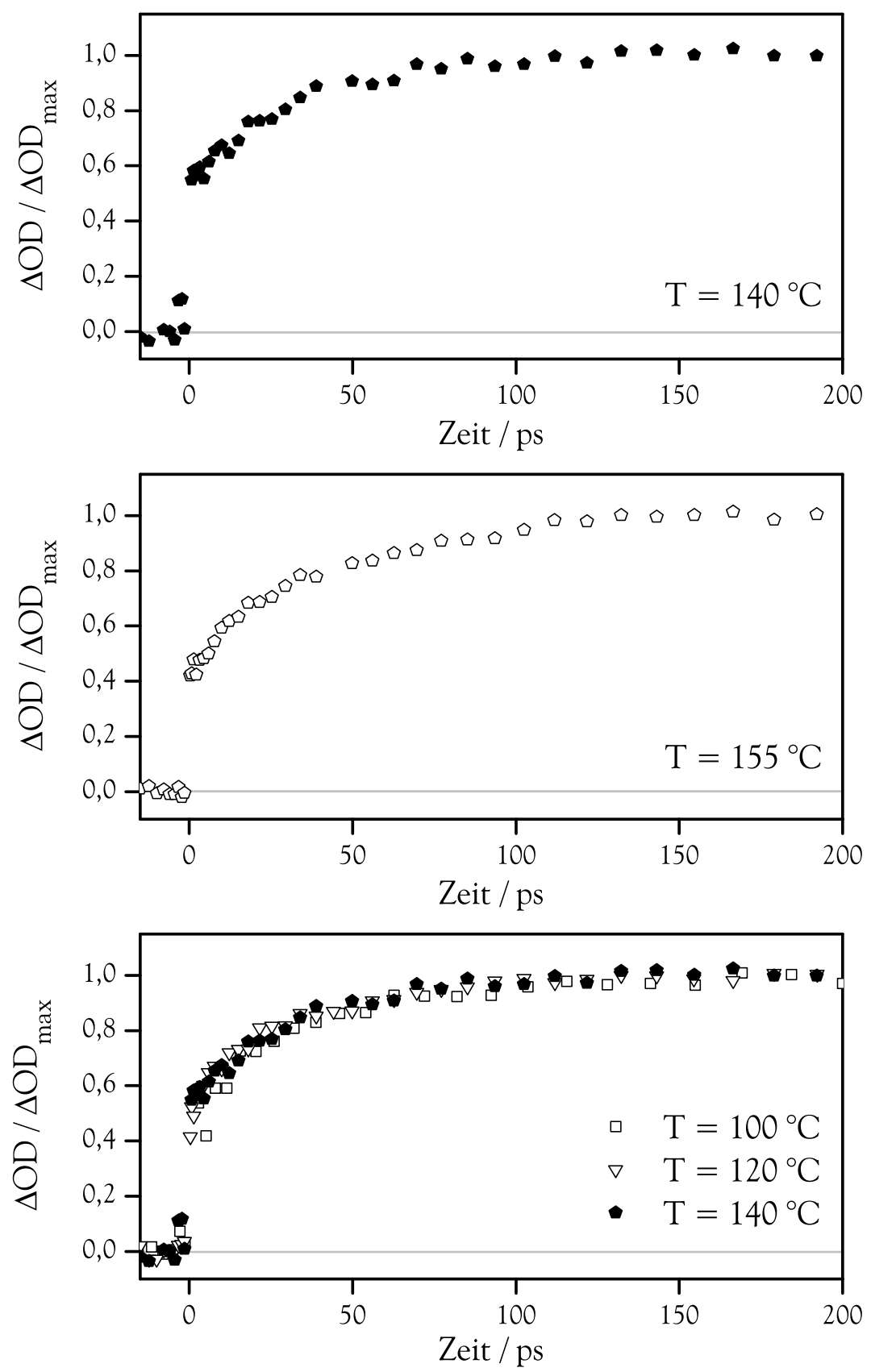

Abbildung 5.4: Transiente Absorption des reinen gasförmigen Benzols bei verschiedenen Temperaturen II ( $\left.\mathrm{p}=0.5 \mathrm{bar}, \lambda_{\text {Pump }}=1670 \mathrm{~nm}, \lambda_{\text {Probe }}=270 \mathrm{~nm}\right)$ 
sem Grund wurden am Referenzsystem Benzol Pump-Probe-Messungen bei fünf verschiedenen Meßtemperaturen im relevanten Temperaturbereich durchgeführt. Der Druck wurde bei jeweils 0.5 bar konstant gehalten, womit die mittlere Zahl von Benzol-Benzol-Stößen $(6.5 \pm 0.6) \cdot 10^{9} \mathrm{~s}^{-1}$ in allen Messungen betrug. Die Anregung erfolgte mit IR-Pulsen der Wellenlänge $1670 \mathrm{~nm}$, womit ein ZOBS mit zweifach populierten $\mathrm{CH}$-Streckschwingungen präpariert wurde. Die transiente Absorption wurde mit Probepulsen der Wellenlänge $270 \mathrm{~nm}$ gemessen.

Die Ergebnisse der temperaturabhängigen Messungen am Benzol sind in den Abb. 5.3 und 5.4 zusammengestellt. Alle Kurven zeigen den gleichen charakteristischen Verlauf, der schon in Abschnitt 5.1.1 erörtert wurde: Einem ersten, nahezu instantanen Anstieg der Absorption auf 0.5 $\Delta \mathrm{OD}_{\max }$ folgt eine zweite, langsamere Dynamik, die nach ca. 100 ps im Signalmaximum endet. Ein nennenswerter Rückgang der Signalintensität konnte im untersuchten Zeitrahmen bis 200 ps bei keiner der fünf Temperaturen beobachtet werden.

Im dritten Graphen der Abb. 5.4 sind die Ergebnisse der Messungen bei 100, 120 und $140^{\circ} \mathrm{C}$ zusammengefaßt. Im Rahmen der Meßgenauigkeit können die drei transienten Absorptionen als identisch aufgefaßt werden. Eine Temperaturabhängigkeit der IVR-Dynamik im relevanten Temperaturbereich kann somit für Benzol ausgeschlossen werden. Für die anderen Modellsysteme ist aufgrund ihrer nahen Verwandschaft zum Benzol (s. Kap. 4) das gleiche Resultat zu erwarten. Die aus Pump-Probe-Messungen bei unterschiedlichen Temperaturen ermittelten IVR-Zeitskalen der verschiedenen Modellsysteme können daher bedenkenlos direkt miteinander verglichen werden.

\section{Druckabhängige Messungen}

Aus IR-Pump-UV-Probe-Messungen an reinem gasförmigem Benzol bei verschiedenen Benzoldrücken kann die Dynamik des ideal-isolierten Moleküls extrapoliert werden, welche mit der hier verwendeten Technik experimentell aufgrund des zu geringen Absorptionsquerschnitts im Niederdruckbereich nicht direkt zugänglich ist.

Bei einer Temperatur von $140^{\circ} \mathrm{C}$ wurden Messungen bei 0.5, 1.0, 2.3 und 3.3 bar Benzoldruck vorgenommen. Höhere Drücke konnten wegen der Dampfdruckbeschränkung für Gasphasenmessungen nicht realisiert werden. Mit einer Anregungswellenlänge von $1670 \mathrm{~nm}$ wurde ein ZOBS mit zwei Quanten in CH-Streckschwingungen populiert. Die Abfragewellenlänge lag, wie bei den Expe- 

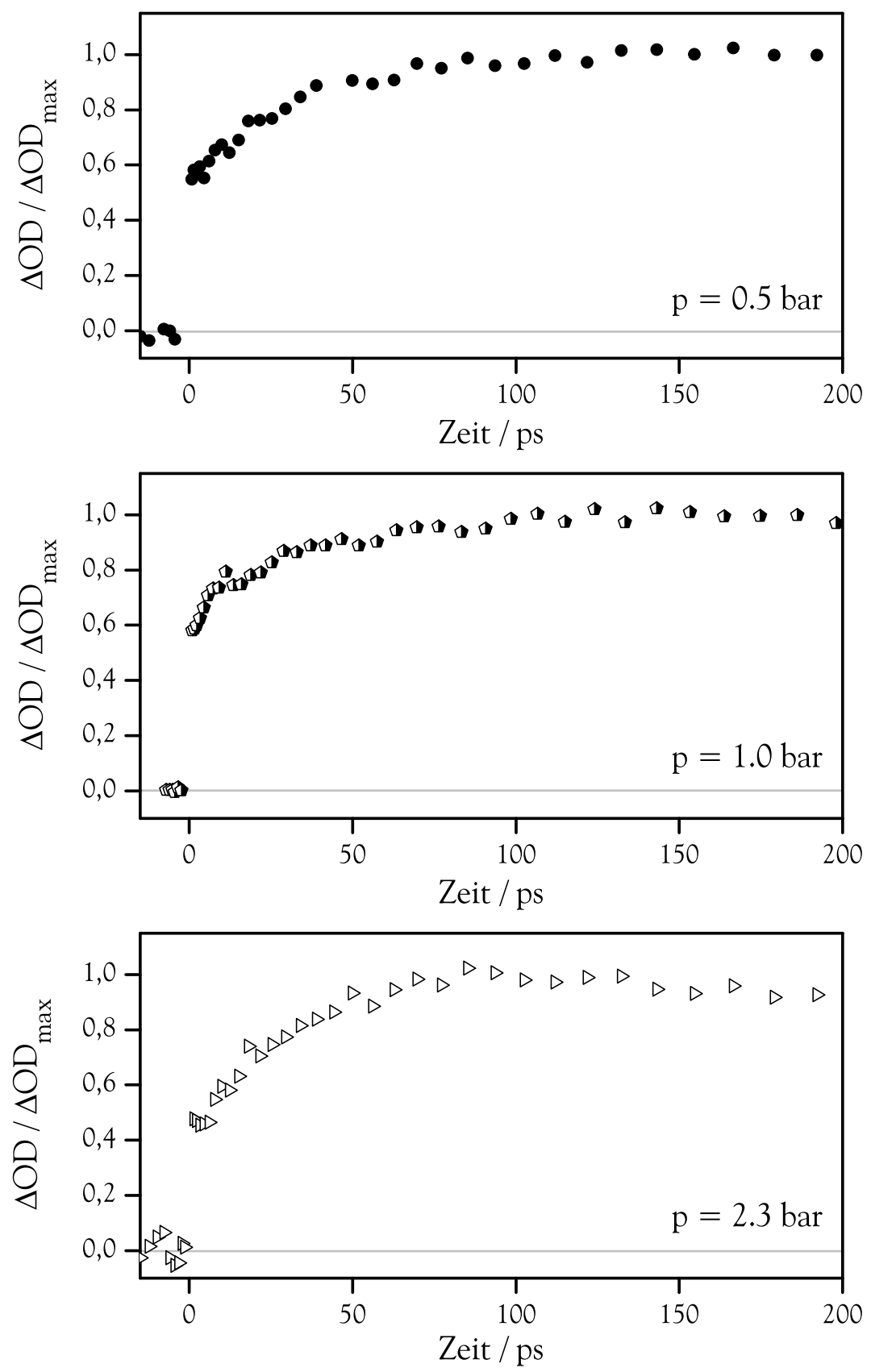

Abbildung 5.5: Transiente Absorption des reinen gasförmigen Benzols bei verschiedenen Benzoldrücken I $\left(\mathrm{T}=140^{\circ} \mathrm{C}, \lambda_{\text {Pump }}=1670 \mathrm{~nm}, \lambda_{\text {Probe }}=270 \mathrm{~nm}\right)$ 

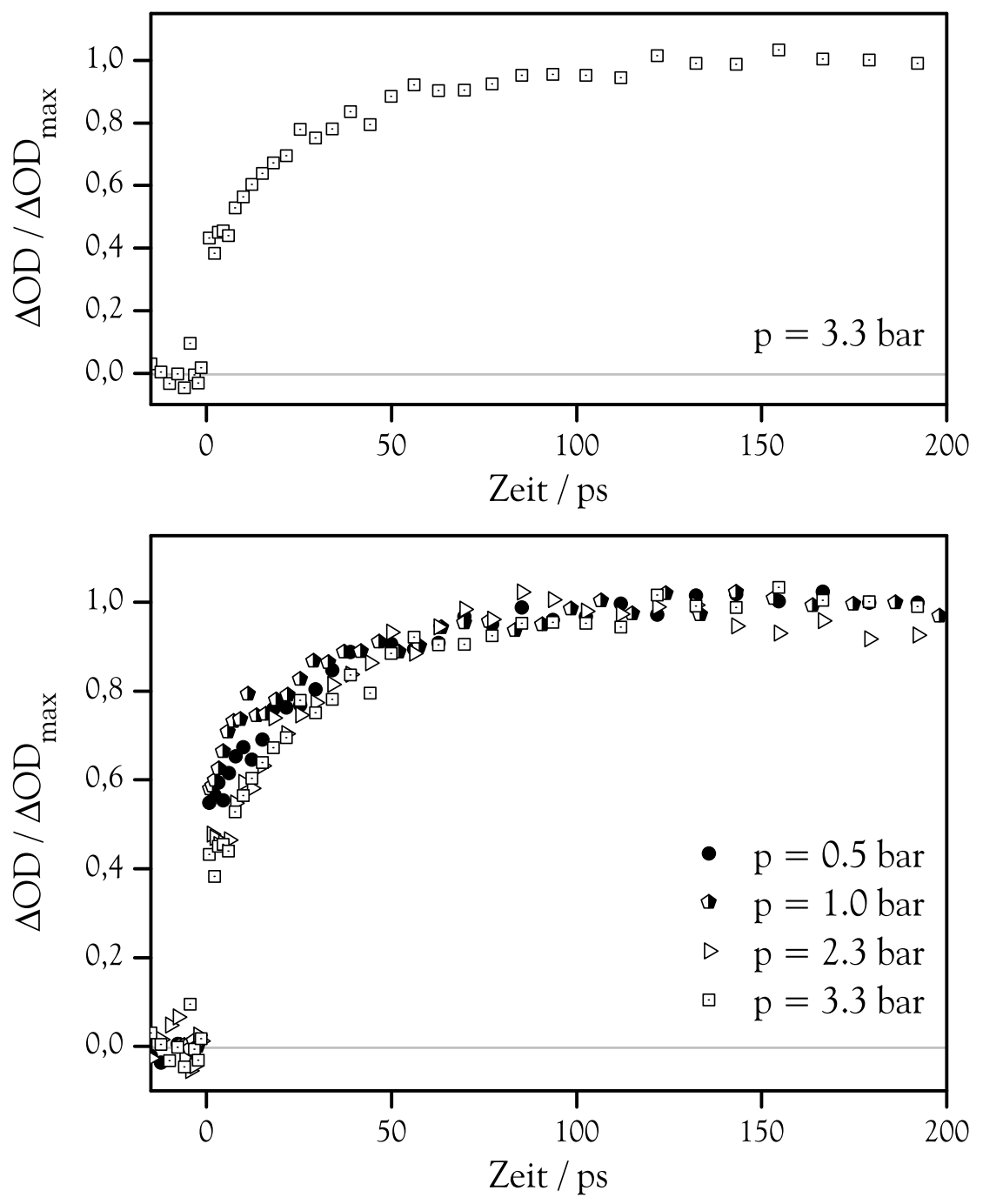

Abbildung 5.6: Transiente Absorption des reinen gasförmigen Benzols bei verschiedenen Benzoldrücken II $\left(\mathrm{T}=140^{\circ} \mathrm{C}, \lambda_{\text {Pump }}=1670 \mathrm{~nm}, \lambda_{\text {Probe }}=270 \mathrm{~nm}\right)$ 
5.1 Gasphasenexperimente an quasi-isolierten Molekülen

rimenten der vorhergehenden Abschnitte, mit $270 \mathrm{~nm}$ auf der langwelligen Flanke des ersten elektronischen Übergangs, so daß der Energiegehalt der niederfrequentesten Benzolschwingungen über deren heiße Banden gemessen wurde.

Die entsprechenden Absorptions-Zeit-Profile sind in den Abb. 5.5 und $5.6 \mathrm{zu}-$ sammengefaßt. Über den gesamten untersuchten Druckbereich bleibt die charakteristisch zweistufige Dynamik der intramolekularen Umverteilung erhalten. Dabei weisen alle Messungen einen unter der experimentellen Zeitauflösung instantanen Anstieg auf mindestens $0.4 \Delta \mathrm{OD}_{\max }$ auf. Nach $100 \mathrm{ps}$ ist die zweite Stufe des IVR-Prozesses in allen Fällen abgeschlossen. Bis 200 ps bleiben die Signalintensitäten dann annähernd konstant auf dem maximalem Wert.

Der direkte Vergleich der druckabhängigen Benzolmessungen im unteren Teil der Abb. 5.6 zeigt geringfügige Unterschiede des langsamen Signalanstiegs bis ca. 50 ps Verzögerungszeit auf. Am deutlichsten weicht die Daten bei 3.3 bar von den übrigen ab, da hier die Absorption zu Beginn der langsamen Dynamik erst $0.4 \Delta \mathrm{OD}_{\max }$ beträgt. Darüber hinaus sind mit bloßem Auge keine eindeutigen druckabhängigen Trends der Dynamik zu quantifizieren. Hier hilft einzig die kinetische Modellierung (s. Kap. 6), mit deren Hilfe die experimentellen IVRZeitskalen quantitativ erfaßt werden können.

\subsubsection{Messungen an Benzol- $d_{1}$}

Monodeuteriertes Benzol- $\mathrm{d}_{1}$ ist unter den vier untersuchten Benzolderivaten das dem Referenzmodellsystem Benzol spektroskopisch am nächsten verwandte Molekül. Ein Vergleich der zeitaufgelösten Pump-Probe-Messungen der beiden Systeme bietet eine elegante Möglichkeit, den Effekt einer signifikanten Symmetriereduktion und damit der damit verbundenen Entartungsaufhebung auf die intramolekulare Schwingungsdynamik zu beleuchten.

\section{Energieabhängige Messungen}

Die transiente Absorption des Benzol- $\mathrm{d}_{1}$ wurde ebenfalls bei zwei verschiedenen Anregungswellenlängen untersucht. Die Messungen erfolgten bei einer Temperatur von $100^{\circ} \mathrm{C}$ und einem Druck von 0.5 bar. Die Probewellenlänge war in Anlehnung an das heiße Banden-Spektrum des undeuterierten Muttermoleküls im Bereich des ersten elektronischen Übergangs auf $270 \mathrm{~nm}$ eingestellt, wie in Abschn. 4.2 erörtert wurde. 

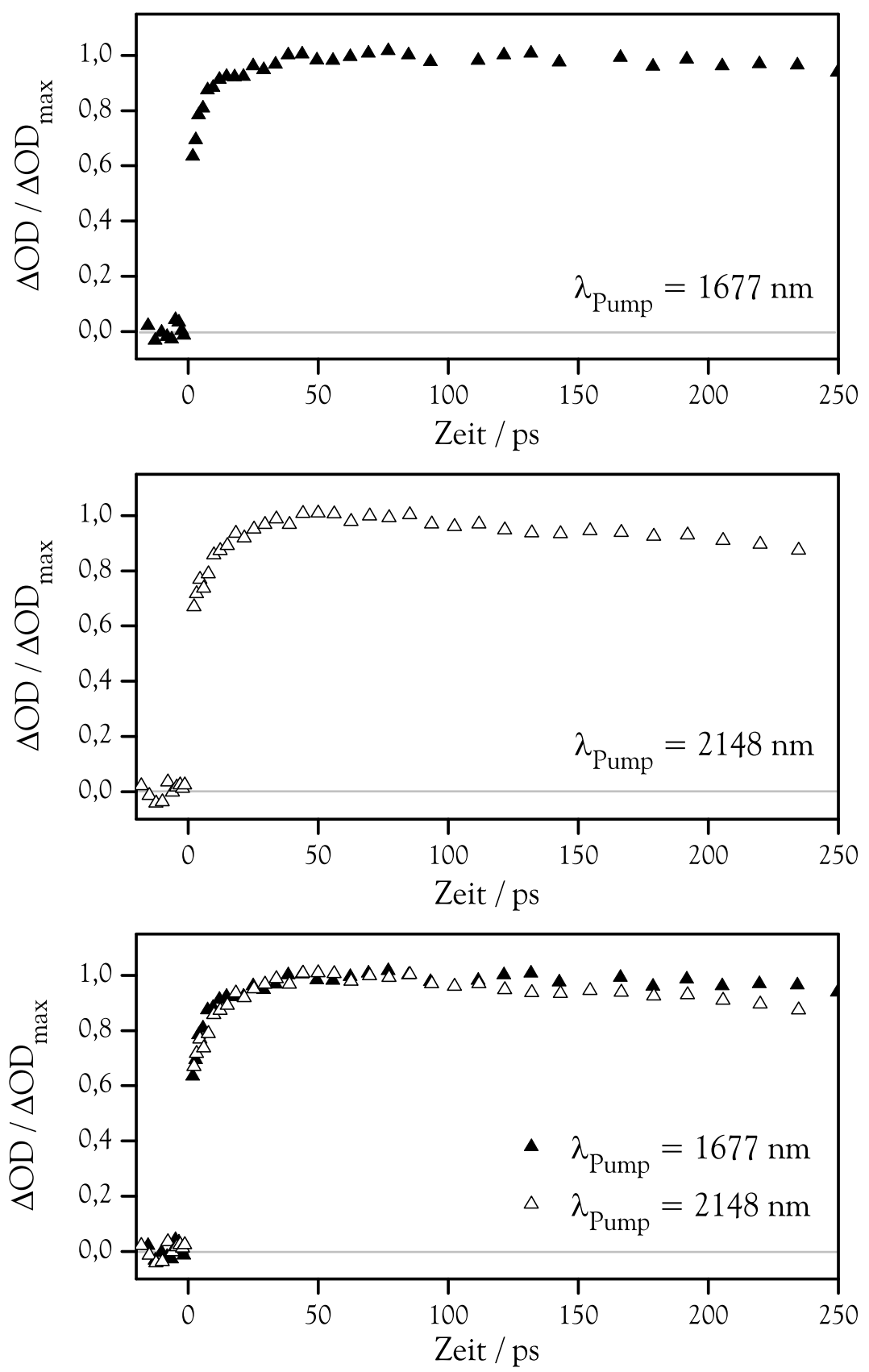

Abbildung 5.7: Transiente Absorption des reinen gasförmigen Benzol- $\mathrm{d}_{1}$ bei verschiedenen Anregungsenergien $\left(\mathrm{T}=100^{\circ} \mathrm{C} ; \mathrm{p}=0.5\right.$ bar, $\left.\lambda_{\text {Probe }}=270 \mathrm{~nm}\right)$ 
Mit der Anregungswellenlänge $\lambda_{\text {Pump }}=1677 \mathrm{~nm}$ konnte die Anregungsenergie im ersten Oberton der CH-Streckschwingungen deponiert werden, wohingegen mit $\lambda_{\text {Pump }}=2148 \mathrm{~nm}$ ein [1:1]-Kombinationston von CH-Streck- mit CH-Biegeschwingungen erzeugt wurde (s. Abschn. 4.2). Abb. 5.7 enthält die entsprechenden einzelnen Absorptions-Zeit-Profile sowie einen direkten Vergleich der Signale. Wie bei Benzol steigt die Änderung der optischen Dichte auch beim monodeuterierten Derivat eindeutig multiexponentiell an. Es existieren für dieses Molekül bei beiden Anregungsenergien mindestens zwei verschiedene IVR-Zeitskalen. Die erste Komponente des Signalanstiegs kann mit der durch den kollinearen Strahlengang bedingten Zeitauflösung nicht vollständig aufgelöst werden. Sie manifestiert sich daher als scheinbar sprunghafter Anstieg der Signalintensität auf ca. 0.6 $\Delta \mathrm{OD}_{\max }$. Hieran schließt sich eine zweite Dynamik an, die nach ca. $50 \mathrm{ps}$

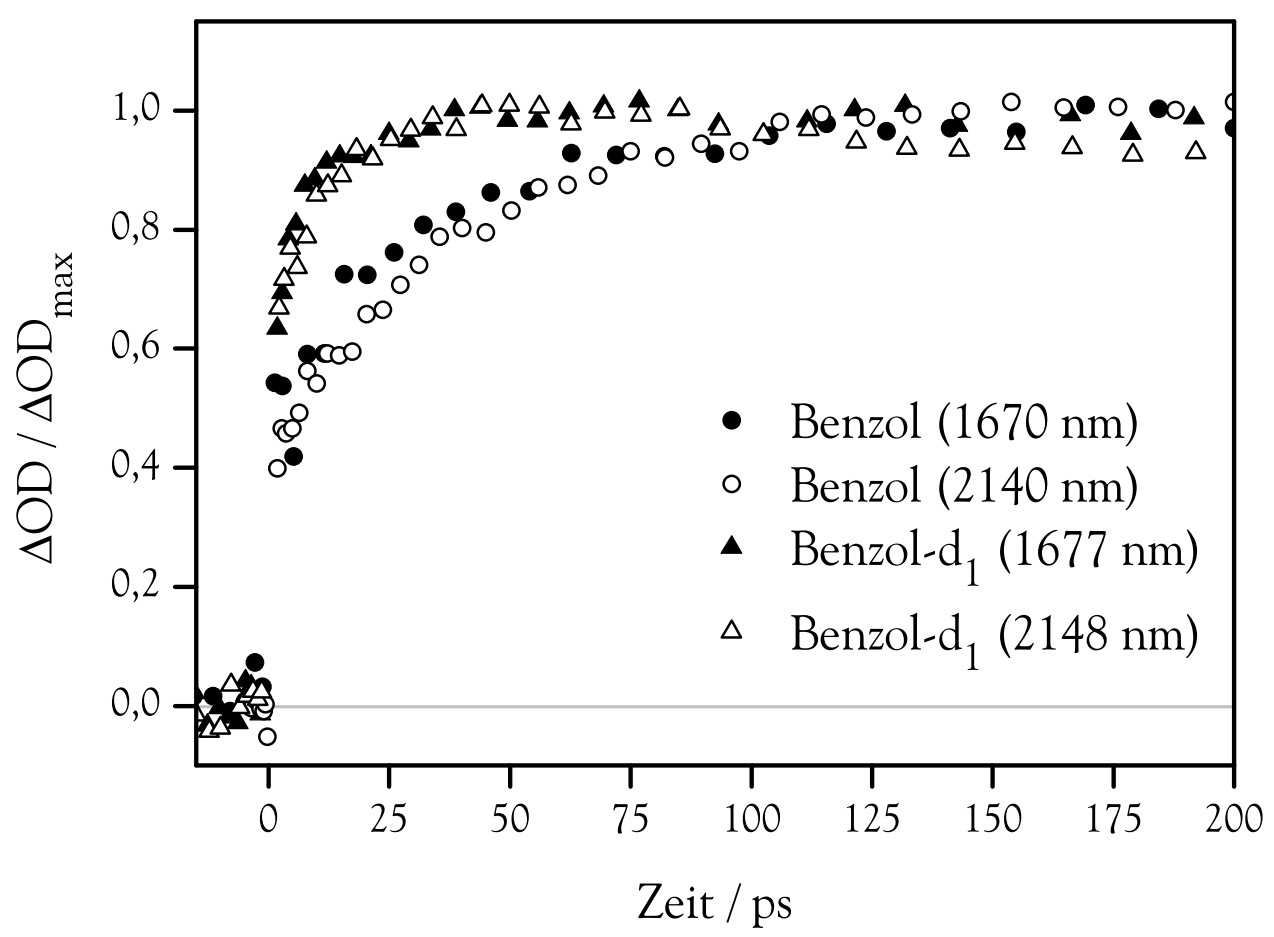

Abbildung 5.8: Vergleich der transienten Absorptionen von Benzol und Benzol- $\mathrm{d}_{1}$ $\left(\mathrm{T}=100^{\circ} \mathrm{C}, \mathrm{p}=0.5 \mathrm{bar}, \lambda_{\text {Probe }}=270 \mathrm{~nm}\right)$ 
abgeschlossen ist. Bei der hohen Anregungsenergie bleibt die Absorptionsänderung auf ihrem maximalen Niveau, während bei der niedrigeren Pumpenergie zusätzlich ein extrem langsamer Signalrückgang zu erkennen ist.

Aus dem Vergleich der transienten Absorptionen ergeben sich sehr ähnliche Trends wie für die entsprechenden Benzolmessungen. Bis ca. 100 ps zeigen die Signale einen nahezu identischen Verlauf, wobei bei der kürzeren Pumpwellenlänge eine minimal schnellere Dynamik aufzutreten scheint. Eine Abnahme der Signalintensität zu langen Zeiten findet wiederum nur bei der längeren Pumpwellenlänge statt.

Die Ergebnisse der Pump-Probe-Messungen an Benzol und Benzol- $\mathrm{d}_{1}$ bei verschiedenen Anregungsenergien sind in Abb. 5.8 zusammengefaßst. Der direkte Vergleich belegt einen deutlich beschleunigenden Einfluß der Monodeuterierung auf die langsame Komponente der intramolekularen Dynamik. Dieser Effekt manifestiert sich sowohl bei der Obertonanregung mit ca. $6000 \mathrm{~cm}^{-1}$ als auch bei der niederfrequentereren Kombinationsmode mit ca. $4500 \mathrm{~cm}^{-1}$ lokalisierter Energie. Desweiteren erscheint das Verhältnis zwischen schneller und langsamer Zeitskala durch die H-D-Substitution zugunsten der ersteren verschoben. Eine Quantifizierung der Auswirkung der Deuterierung erfolgt anhand eines mehrstufigen kinetischen Modells in Kap. 6.

\subsubsection{Messungen an Chlorbenzol}

In Pump-Probe-Messungen an monochloriertem Benzol kann die Beeinflussung des Schwingungsenergietransfers durch ein Schweratom in geminaler Position zum phenylischen Kohlenstoffring untersucht werden.

Wegen seines vergleichsweise hohen Siedepunktes $\left(\mathrm{T}_{\mathrm{s}}=132^{\circ} \mathrm{C}\right)$ wurden die Experimente an Chlorbenzol bei $140^{\circ} \mathrm{C}$ durchgeführt, um einen Substanzdruck von 0.5 bar in der Zelle sicherstellen zu können. Als ZOBS wurde der erste Oberton der $\mathrm{CH}$-Streckschwingung mit ca. $6000 \mathrm{~cm}^{-1}$ erzeugt, die Anregungswellenlänge hierfür betrug $1663 \mathrm{~nm}$. Die Abfrage der Dynamik erfolgte mit Pumppulsen der Wellenlänge $283 \mathrm{~nm}$ im Bereich der heißen Banden des ersten elektronischen Übergangs.

Im Verlauf der Messungen traten nicht näher identifizierbare Ablagerungen auf den Zellenfenstern auf, die es erforderten, die Meßzelle kontinuierlich senkrecht zum Strahlengang zu versetzen. So konnte eine reine Gasphasenabsorption ge- 


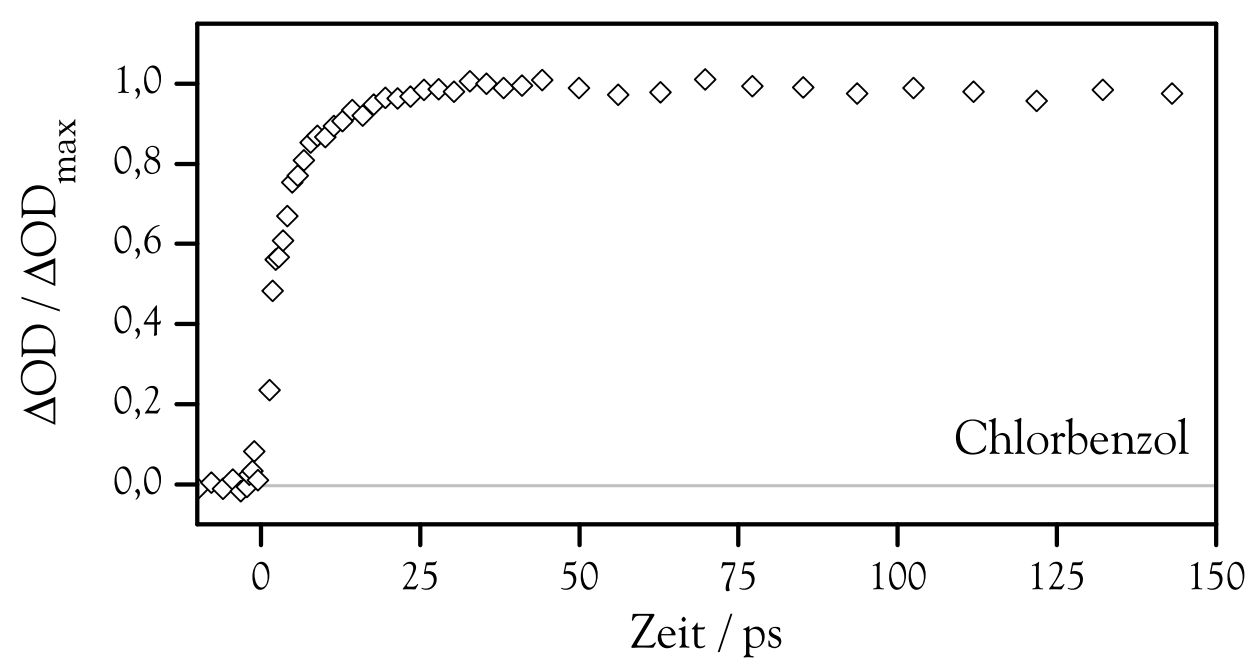

Abbildung 5.9: Transiente Absorption des reinen gasförmigen Chlorbenzols $\left(\mathrm{T}=140^{\circ} \mathrm{C}, \mathrm{p}=0.5 \mathrm{bar}, \lambda_{\text {Pump }}=1663 \mathrm{~nm}, \lambda_{\text {Probe }}=283 \mathrm{~nm}\right)$

währleistet werden. Das endgültige Signal-zu-Rausch-Verhältnis darf trotz dieser Schwierigkeiten als sehr gut bezeichnet werden.

Die Dynamik des Chlorbenzols wurde zwischen 0 und 150 ps untersucht. Das Resultat aller gemittelten Messungen zeigt Abb. 5.9. Der Anstieg der transienten Absorption konnte nicht vollständig aufgelöst werden. Eine sprunghafte Zunahme wie im Falle des Benzols und Benzol- $\mathrm{d}_{1}$ tritt beim Chlorbenzol nicht auf; dennoch erscheint auch hier der Signalverlauf zwischen 0 und $1 \Delta \mathrm{OD}_{\max }$ multiexponentiell. Die intramolekulare Umverteilung läßt die Signalhöhe innnerhalb der ersten 30 ps auf ihren Maximalwert ansteigen, auf dem sie für den Rest der untersuchten Zeitspanne verbleibt. Die Monochlorierung beschleunigt somit ebenso wie die Monodeuterierung den IVR-Prozess im Vergleich zum Referenzmolekül Benzol.

\subsubsection{Messungen an Toluol}

Toluol wurde als Modellsystem gewählt, um den Einfluß eines internen Rotators auf die intramolekulare Schwingungsdynamik in der Gasphase zu studieren. Der geringe Absorptionsquerschnitt dieser Spezies erforderte einen Mindestdruck von 


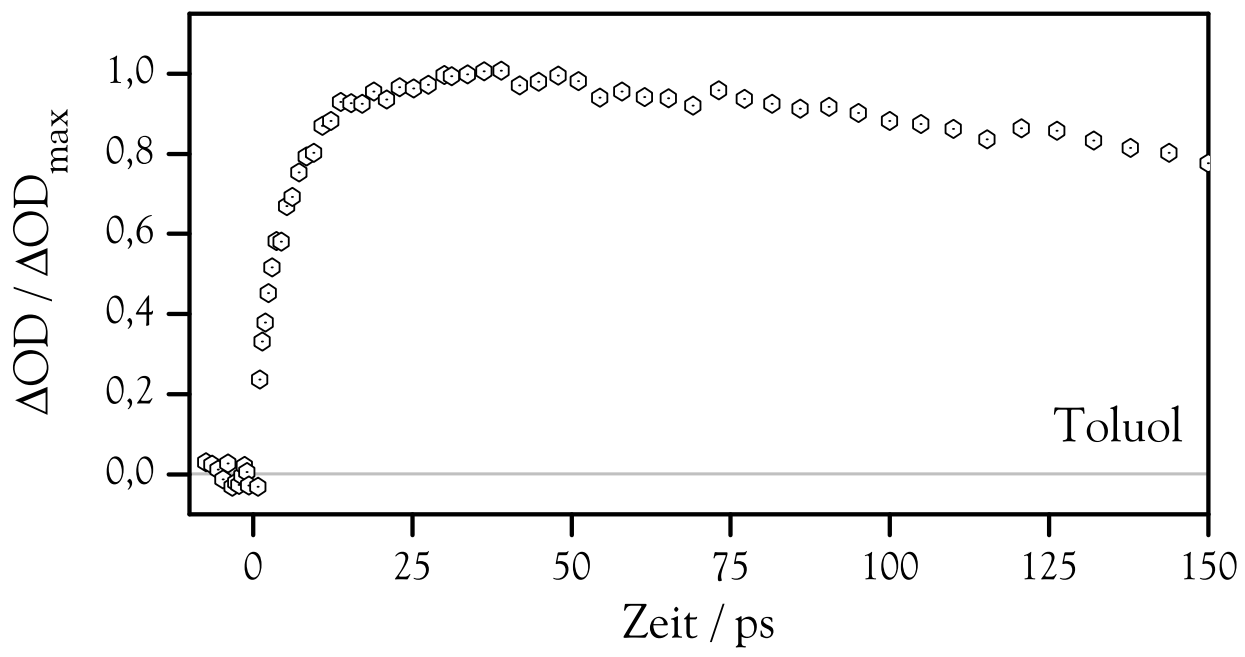

Abbildung 5.10: Transiente Absorption des reinen gasförmigen Toluols $\left(\mathrm{T}=145^{\circ} \mathrm{C}, \mathrm{p}=0.8 \mathrm{bar}, \lambda_{\text {Pump }}=1678 \mathrm{~nm}, \lambda_{\text {Probe }}=280 \mathrm{~nm}\right)$

0.8 bar bei den Pump-Probe-Messungen. Obwohl Toluol unter Normaldruck bei $110^{\circ} \mathrm{C}$ siedet, war eine Mestemperatur von $145^{\circ} \mathrm{C}$ vonnöten, um etwaige Kondensationseffekte auf den Zellfenstern zu vermeiden. Unter diesen Bedingungen konnte ein Signal-zu-Rausch-Verhältnis von über 30 erzielt werden.

Die Anregung des ZOBS erfolgte durch IR-Pulse mit $1678 \mathrm{~nm}$ Wellenlänge, so daß hauptsächlich der erste Oberton der phenylischen $\mathrm{CH}$-Streckschwingungen populiert wurde. Eine Delokalisierung der Anregungsenergie auf die methylischen $\mathrm{CH}$-Bindungen kann allerdings nicht ausgeschlossen werden (vgl. spektroskopische Eigenschaften dieses Modellsystems, Abschn. 4.4). Der Fortschritt der Energieumverteilung wurde anhand des Energiegehalts niederfrequenter FC-aktiver Toluolmoden verfolgt. Hierfür wurden UV-Probepulse mit einer Wellenlänge von $280 \mathrm{~nm}$ eingesetzt, welche von heißen Banden im $\mathrm{S}_{1} \leftarrow \mathrm{S}_{0}$-Übergang des Moleküls absorbiert werden.

Abb. 5.10 stellt das Ergebnis der gemittelten Pump-Probe-Messungen an Toluol dar. Das Signal besitzt den für Gasphasendaten typischen Verlauf mit vergleichsweise schnellem Anstieg der Intensität und relativ langsamem Rückgang derselben. Bei ca. 40 ps liegt das Maximum der Absorption. Der Anstieg der Ab- 
5.1 Gasphasenexperimente an quasi-isolierten Molekülen

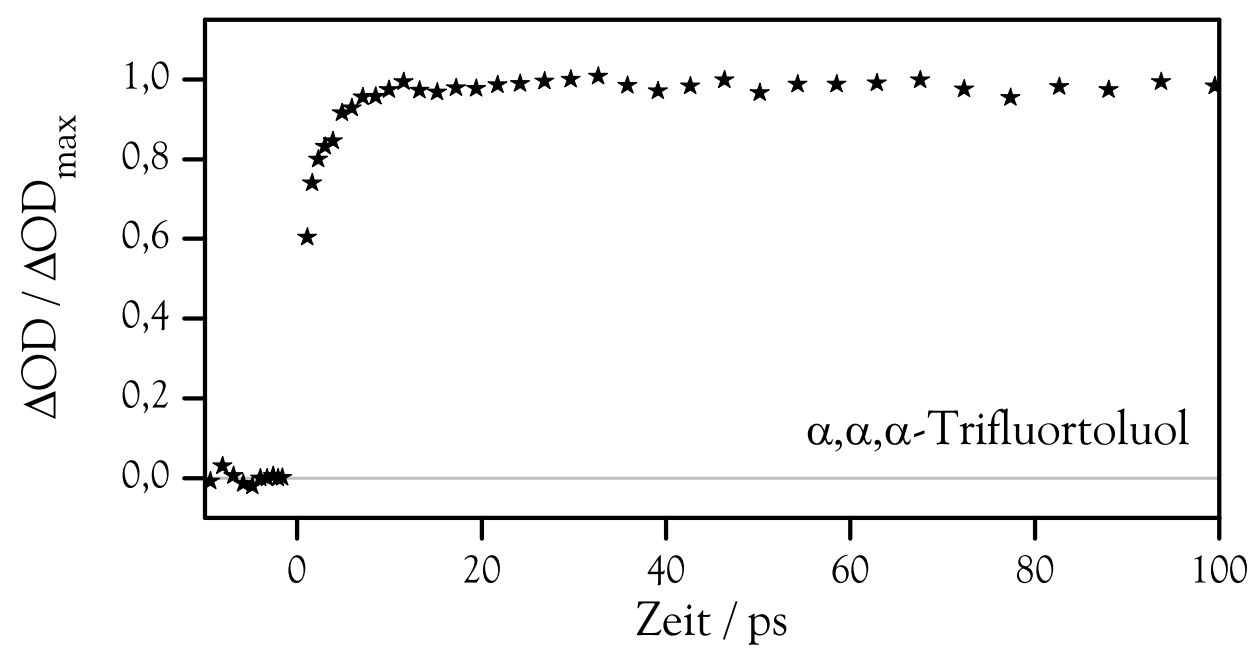

Abbildung 5.11: Transiente Absorption des reinen gasförmigen $\alpha, \alpha, \alpha$-Trifluortoluols $\quad\left(\mathrm{T}=145^{\circ} \mathrm{C}, \quad \mathrm{p}=0.8 \mathrm{bar}, \quad \lambda_{\text {Pump }}=1678 \mathrm{~nm}\right.$, $\left.\lambda_{\text {Probe }}=280 \mathrm{~nm}\right)$

sorptionsänderung konnte in diesem Fall nahezu vollständig aufgelöst werden. Er weist - mit bloßem Auge betrachtet - einen monoexponentiellen Verlauf auf. Die Abnahme des Signals zwischen 50 und 150 ps zeigt dagegen auf den ersten Blick ein quasi-lineares Verhalten. Eine genauere Untersuchung der intra- und intermolekularen Dynamik auf (multi-)exponentielle Zeitskalen erfolgt im Rahmen der Auswertung der Pump-Probe-Experimente in Kap. 6.

\subsubsection{Messungen an $\alpha, \alpha, \alpha$-Trifluortoluol}

Die Experimente an $\alpha, \alpha, \alpha$-Trifluortoluol stellen die konsequente Fortsetzung des Substitutionsmusters eines internen Rotators dar, weil hier zusätzlich eine signifikant vergrößerte Zustandsdichte durch die niederfrequenten CF-Moden gegeben ist.

Der Fokus des Experiments lag wie beim Toluol auf der intramolekularen Energieumverteilung aus dem ersten Oberton der arylischen $\mathrm{CH}-$ Streckschwingungen heraus. Der entsprechende ZOBS wurde durch Anregungspulse mit $\lambda_{\text {Pump }}=$ 
Kapitel 5 Ergebnisse

$1660 \mathrm{~nm}$ generiert. Die Wellenlänge der UV-Abfragepulse war mit $272 \mathrm{~nm}$ deutlich größer als die des (0,0)-Übergangs, so daß ausschließlich schwingungsheiße Moleküle zur Absorption beitrugen (s. Abschn. 4.5).

Der Siedepunkt des $\alpha, \alpha, \alpha$-Trifluortoluols unter Normaldruck liegt bei $101^{\circ} \mathrm{C}$. Für einen Substanzdruck von 0.5 bar unter Ausschluß von Kondensationseffekten auf den Zellfenstern mußte die Zelle dennoch auf $125^{\circ} \mathrm{C}$ temperiert werden.

Das Resultat der gemittelten transienten Absorptionsmessungen an $\alpha, \alpha, \alpha$-Trifluortoluol ist in Abb. 5.11 gezeigt. Der Verlauf des Signals ähnelt dem der übrigen monosubstituierten Modellsysteme bei Obertonanregung sehr. Aufgrund der durch kollinearen Strahlengang und lange optische Weglänge begrenzten Zeitauflösung (s. Abschn. 3.2.4) ist die intramolekulare Dynamik

- der Signalanstieg - nicht vollständig erfaßst. Vielmehr nimmt die Änderung der optischen Dichte innerhalb der ersten Pikosekunde scheinbar sprunghaft auf 0.6 $\Delta \mathrm{OD}_{\max }$ zu. Dem schlieft sich ein zweiter IVR-Schritt an, der in einem Maximum des Signals schon nach ca. 15 ps endet. Von den fünf aromatischen Modellsystemen weist $\alpha, \alpha, \alpha$-Trifluortoluol somit die schnellste intramolekulare Dynamik auf. Für den restlichen untersuchten Zeitraum verbleibt die transiente Absorption auf einem Plateau bei $1.0 \Delta \mathrm{OD}_{\max }$.

\subsubsection{Vergleich der fünf aromatischen Modellsysteme}

Dieser erste Teil des Ergebniskapitels, der den Gasphasenexperimenten an reinen Substanzen gewidmet ist, soll mit einem phänomenologischen Vergleich der transienten Absorptionsspuren aller fünf aromatischer Modellsysteme abgeschlossen werden. Dabei beschränkt sich die Gegenüberstellung auf die intramolekulare Dynamik, die nach $2 v_{\mathrm{CH}^{-}}$Obertonanregung mit ca. $6000 \mathrm{~cm}^{-1}$ beobachtet wurde. Die Resultate der Messungen an Benzol, Benzol- $\mathrm{d}_{1}$, Chlorbenzol, Toluol und $\alpha, \alpha, \alpha$-Trifluortoluol sind in Abb. 5.12 gesammelt. Die Bedingungen der Einzelmessungen können den vorigen Abschnitten entnommen werden.

Ein erster Blick auf die fünf Absorptionssignale zeigt, daß Benzol sich in seiner Dynamik deutlich von den anderen Modellsystemen unterscheidet. Die Substitution eines H-Atoms durch ein anderes Atom bzw. durch eine chemische Gruppe führt in allen Fällen zu einer Beschleunigung des intramolekularen Energieflusses. Bei $\alpha, \alpha, \alpha$-Trifluortoluol manifestiert sich dieser Effekt am stärksten. Darüber 
hinaus herrscht eine auffallende Ähnlichkeit zwischen den Signalen der Derivate, besonders bei Benzol- $\mathrm{d}_{1}$, Chlorbenzol und Toluol.

Ein genauerer Vergleich der IVR-Zeitskalen ist nach einer quantitativen Modellierung der Pump-Probe-Signale möglich, wie sie in Kap. 6 vorgestellt wird. Die hieraus gewonnnenen Zeitkonstanten dienen als Grundlage der Diskussion über den Zusammenhang zwischen molekularer Konstitution und intramolekularer Energieumverteilung in Kap. 7.

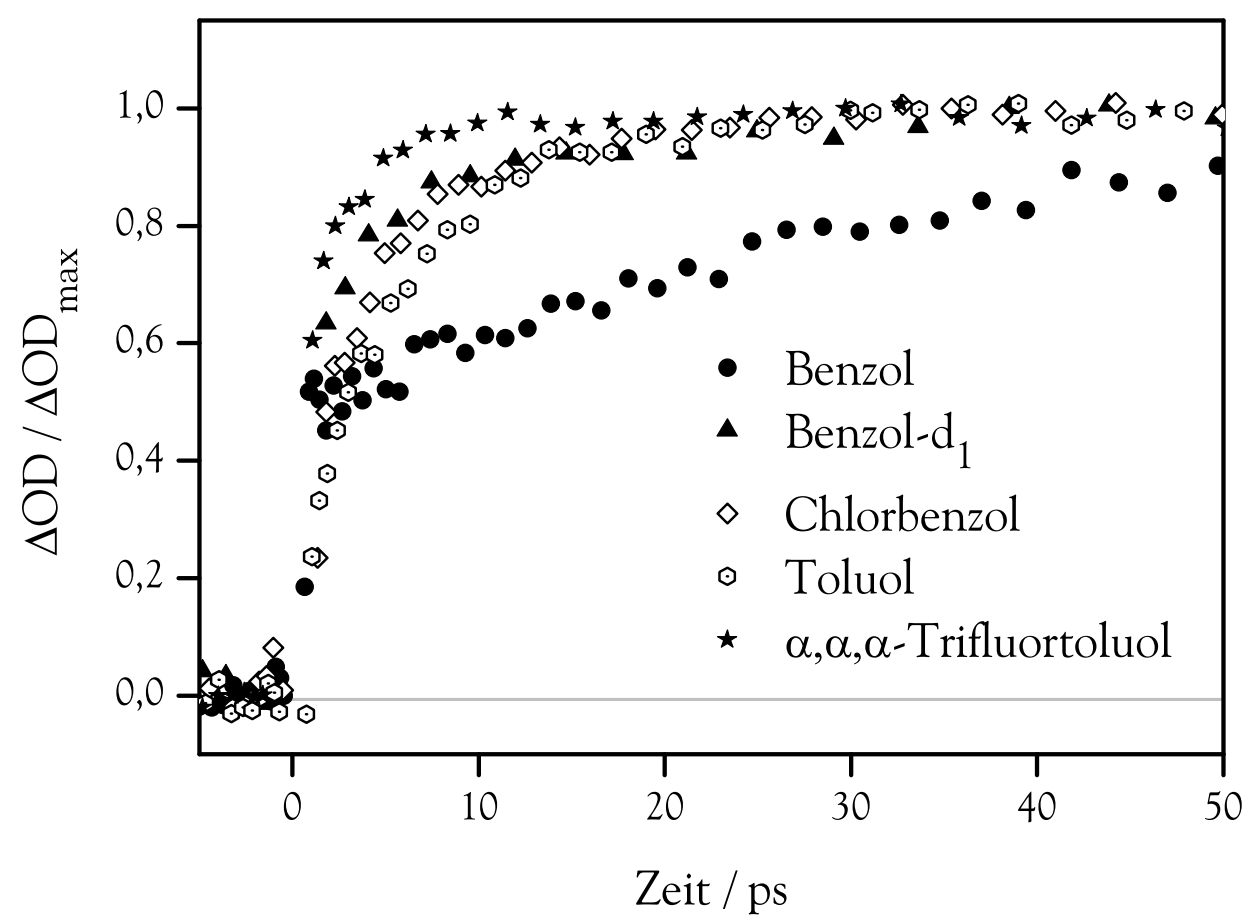

Abbildung 5.12: Vergleich der transienten Absorptionen der fünf aromatischen Modellsysteme nach $2 v_{\mathrm{CH}^{-}}$Obertonanregung 


\subsection{Experimente in unterschiedlichen molekularen Umgebungen}

In den Experimenten, die im folgenden vorgestellt werden, wurde die molekulare Umgebung eines lokal angeregten gasförmigen Aromaten variiert. Als Modellsystem, dessen intra- und intermolekulare Dynamik mit der IR-Pump-UVProbe-Technik beobachtet wird, fungierte in allen diesen Versuchen das Benzol. Ein Vorteil dieses Referenzsystems besteht in seiner auffallend langsamen sekundären IVR-Zeitskala unter quasi-isolierten Bedingungen. Diese gewährleist, daß eine eventuelle Beschleunigung der Umverteilung von bis zu einer Größenordnung immer noch zeitaufgelöst untersucht werden kann.

Der helle Zustand nullter Ordnung wurde jeweils mit Pumppulsen der Wellenlänge $1670 \mathrm{~nm}$ präpariert; es handelte sich somit jedesmal um den obertonartigen $2 v_{\mathrm{CH}^{-}}$Schwingungszustand. Die Abfrage der Dynamik erfolgte analog zu den Messungen am quasi-isolierten Molekül mit $270 \mathrm{~nm}$-Probepulsen. Der Benzoldruck betrug in allen Experimenten 0.5 bar.

Da eine selektive Anregung und Abfrage des Benzols erwünscht war, konnten nur solche Badgase und überkritische Lösungsmittel verwendet werden, die bei den Wellenlängen von Pump- und Probepuls nicht absorbieren. Dies schloß sämtliche Spezies mit CH-Bindungen als Stoßpartner aus. Um trotzdem polyatomare Kohlenwasserstoffe als molekulare Umgebung verwenden zu können, wurde auf deren perhalogenierte Derivate zurückgegriffen. Dies hat sich bereits bei Untersuchungen in kondensierter Phase bewährt [93-95].

Die Stoßgas-Messungen stellten sich aufgrund unerwarteteter Entmischungsphänomene durchweg als experimentell sehr anspruchsvoll heraus. Eine Vielzahl von Stoßgasen, z. B. Stickstoff, konnte überhaupt nicht in die Studie inkorporiert werden, da es zu Schlierenbildung und anderen anomalen Rauschquellen in der Zelle kam, die eine Absorptionsmessung unmöglich machten. Diese Erscheinungen konnten nur in wenigen Fällen durch Verwendung der Zelle für überkritische Lösungsmittel, mit der eine aktive Durchmischung der Komponenten möglich ist (s. Abschn. 3.3.2), unterdrückt werden. Allerdings wirkte sich die kürzere Schichtdicke dieser Zelle empfindlich auf die Signalintensität aus. Das exzellente Signal-zu-Rausch-Verhältnis der Messungen an den reinen Substanzen ließ sich aus diesen Gründen leider nur in den wenigsten Fällen reproduzieren. 


\subsubsection{Messungen mit Helium}

Das Edelgas Helium stellt unter den fünf untersuchten Badgasen den leichtesten monoatomaren Stoßpartner dar. Es war möglich, Messungen bei einem Badgasdruck von 158 bar und einer Temperatur von $100{ }^{\circ} \mathrm{C}$ durchzuführen. Unter diesen Bedingungen berechnet sich die Lennard-Jones-Stoßzahl von Benzol-HeliumStößen zu $1.4 \mathrm{ps}^{-1}$.

Das Resultat der Pump-Probe-Experimente an diesem System ist im obersten Graphen der Abb. 5.13 aufgetragen. Der Verlauf zeigt einen relativ schnellen Anstieg der Signalintensität innerhalb von 50 ps nach der Anregung auf ihren maximalen Wert, gefolgt von einem vergleichsweise langsamen Rückgang des Signals. Obwohl der durch die intramolekulare Energieumverteilung verursachte Anstieg nicht vollständig aufgelöst werden konnte, ist eindeutig die multiexponentielle Form zu erkennen, welche auch für die transiente Absorption des reinen Benzols charakteristisch ist.

\subsubsection{Messungen mit Argon}

Als zweites Edelgas wurde Argon eingesetzt. Bei einer Meßtemperatur von $100{ }^{\circ} \mathrm{C}$ wurde ein Gasdruck von 200 bar eingestellt, um eine mit den Helium-Messungen vergleichbare Stoßfrequenz von 1.1 Benzol-Argon-Stößen pro ps zu erhalten.

Im mittleren Teil der Abb. 5.13 ist das Ergebnis dieser Experimente dargestellt. Das Absorptions-Zeit-Profil ist dem des reinen Benzols (s. Abb. 5.2, oben) sehr ähnlich. Einem innerhalb der experimentellen Zeitauflösung nicht besser aufzulösenden Anstieg des Signals auf 0.4 $\Delta \mathrm{OD}_{\max }$ schließst sich eine zweite, annähernd monoexponentielle IVR-Komponente an. Die Kurve erreicht nach ca. 100 ps ihr Maximum, woraufhin die Signalintensität auf diesem Wert verbleibt. Erst nach 300 ps deutet sich ein leichter Signalrückgang an. Somit bleibt auch mit Argon als Stoßpartner die benzoltypische zweistufige IVR-Dynamik bestehen.

\subsubsection{Messungen mit Kohlendioxid}

Kohlendioxid wurde bereits zuvor von unserer Arbeitsgruppe als überkritisches Lösungsmittel für Benzol verwendet [43,96], um die Relation zwischen IVR- bzw. VET-Geschwindigkeit und reduzierter Lösungsmitteldichte zu erforschen. Da es sich um ein polyatomares Badgas mit zwei Rotations- und vier Schwingungsfrei- 


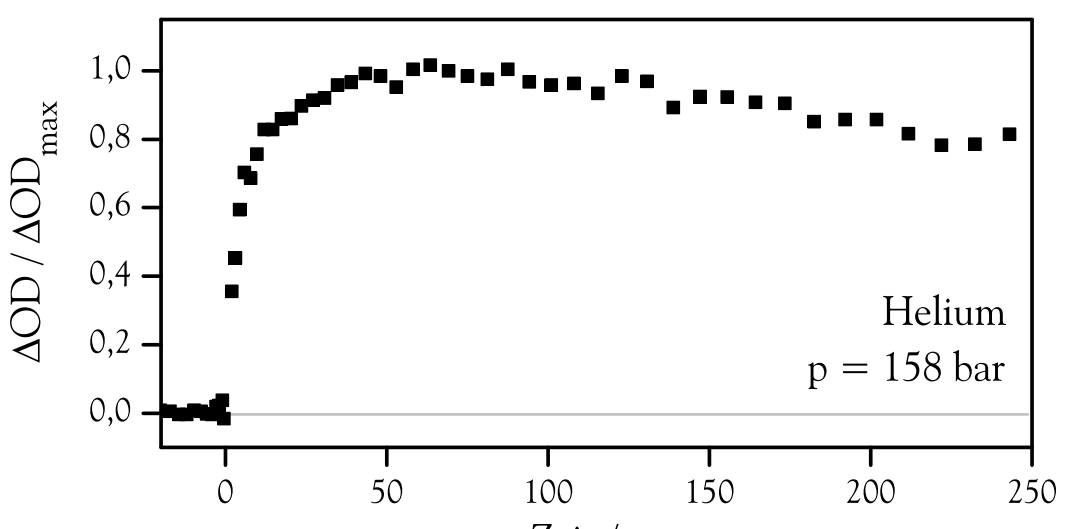

Zeit / ps
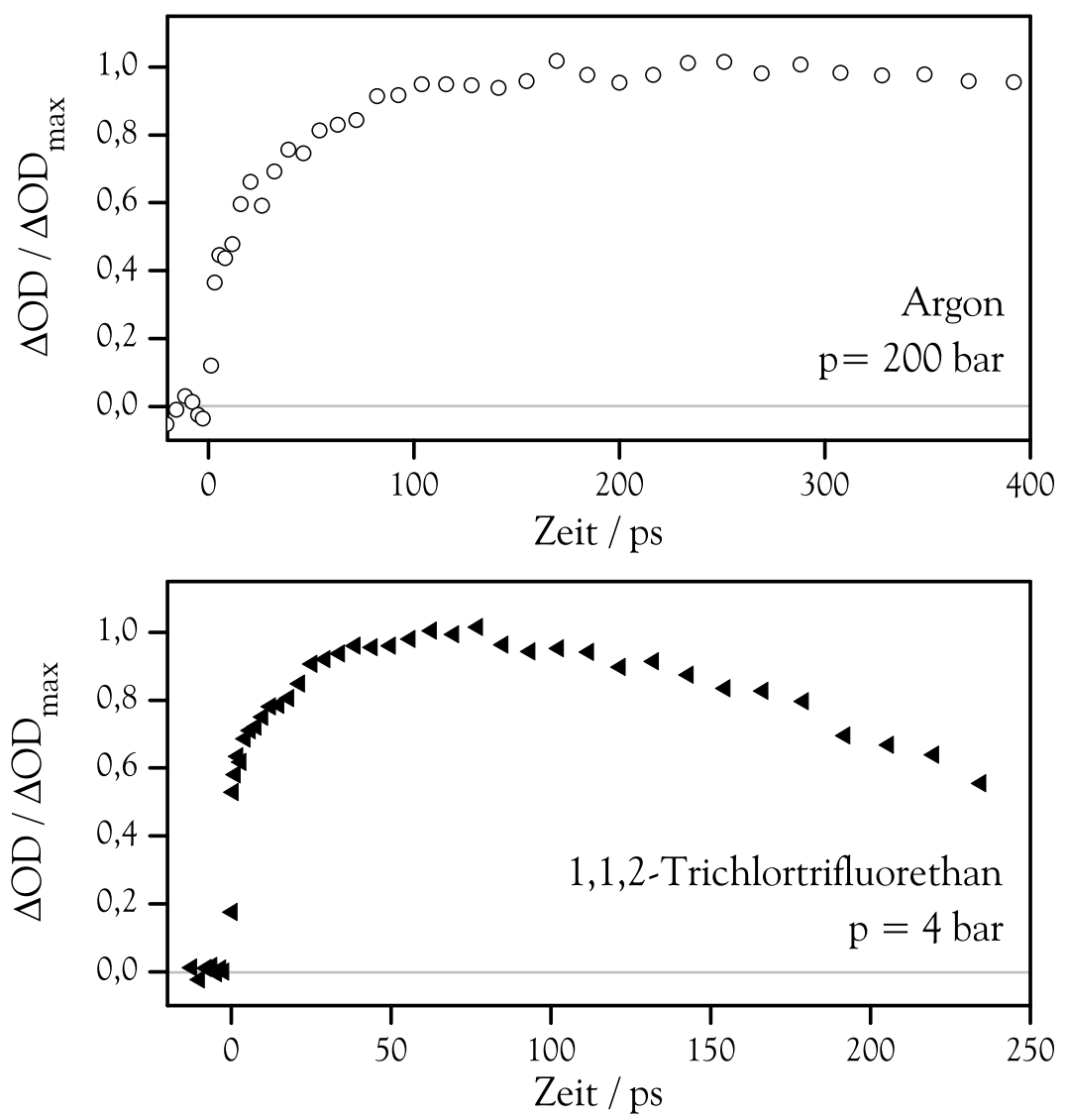

Abbildung 5.13: Transiente Absorption von Benzol in Helium, Argon und 1,1,2-Trichlortrifluorethan 
heitsgraden handelt, sind - im Gegensatz zu den Edelgasen Helium und Argon - bei diesem Stoßpartner prinzipiell auch V-R- und/oder V-V-Energietransfer möglich.

Mit der damaligen Technik konnten nur reduzierte $\mathrm{CO}_{2}$-Dichten von mehr als 0.7 untersucht werden. Im Rahmen dieser Arbeit wurde u. a. versucht, diesen Datensatz um Messungen in stark verdünnter Gasphase zu erweitern. Dies war jedoch nur in eingeschränktem Maße möglich, da bei $\mathrm{CO}_{2}$-Drücken von mehr als 20 bar die bereits erwähnten Entmischungseffekte auftraten, die zu einer erheblichen Verschlechterung der Signalqualität führten.

Drei Ergebnisse dieser Experimente sind in Abb. 5.14 dargestellt. Es handelt sich um die Messungen in gasförmigem Kohlendioxid bei 10 bzw. 18 bar (oberer und mittlerer Graph) und in überkritischer Phase bei 100 bar $\mathrm{CO}_{2}$-Partialdruck (unterer Graph). Diese Drücke entsprechen reduzierten Dichten von 0.03, 0.06 und 0.4. Die Temperatur in der Meßzelle betrug jeweils $100^{\circ} \mathrm{C}$. Es wurden nicht mehr als 0.5 bar Benzol eingefüllt, um die Anzahl von Benzol-Benzol-Selbststößen vernachlässigbar gering zu halten.

Die beiden unter Gasphasenbedingungen erhaltenen Absorptions-Zeit-Profile weisen unverkennbare Ähnlichkeiten mit den Messungen an reinem Benzol (z. B. Abb. 5.5) auf. In beiden Fällen erreicht das Signal nach ca. 70 ps ein Plateau bei $1.0 \Delta \mathrm{OD}_{\max }$. Der Signalanstieg besitzt wiederum den typisch multiexponentiellen Charakter, der bereits in den vorigen Abschnitten festgestellt wurde. In der Messung bei 10 bar nimmt die Signalintensität innerhalb der ersten Pikosekunde scheinbar instantan um $0.5 \Delta \mathrm{OD}_{\max } \mathrm{zu}$, wohingegen diese erste Komponente bei 18 bar ca. 5 ps dauert.

Im Absorptions-Zeit-Profil bei 100 bar sind sowohl die Zeitskala des Signalanstiegs als auch die des Signalrückgangs im Vergleich zu den beiden anderen Messungen verkürzt. Schon nach ca. 20 ps erreicht die Signalintensität ihr Maximum und geht anschließend bis zur Verzögerungszeit von 200 ps fast auf die Grundlinie zurück. Aufgrund der hohen und fluktuierenden Dichte des Mediums war es nicht möglich, den Anstieg der Absorption vollständig aufzulösen oder ein besseres Signal-zu-Rausch-Verhältnis zu erreichen. Dennoch sind die Unterschiede zu den übrigen $\mathrm{CO}_{2}$-Transienten deutlich zu erkennen. 

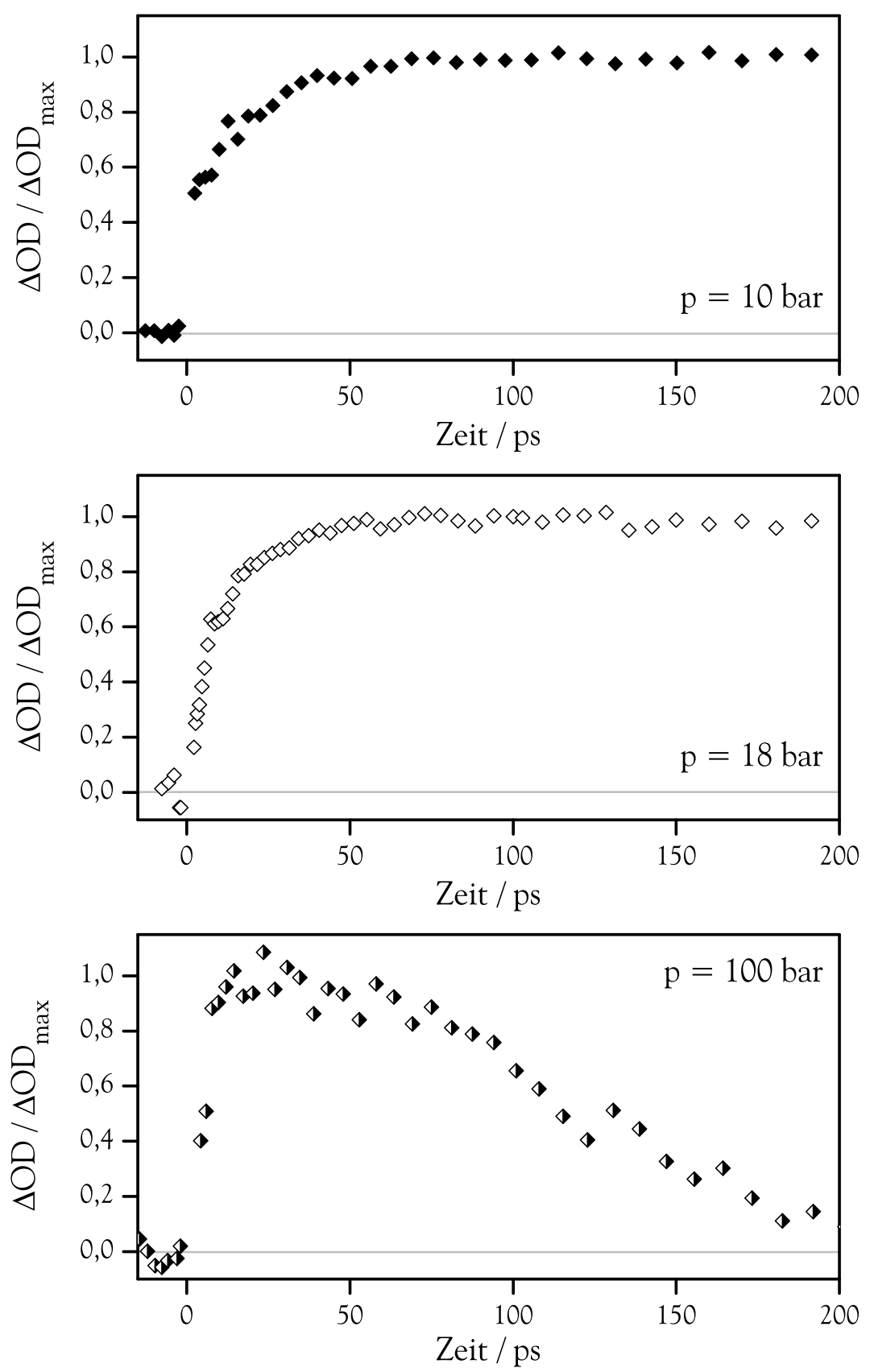

Abbildung 5.14: Transiente Absorption von Benzol in $\mathrm{CO}_{2}$ bei verschiedenen Badgasdrücken 


\subsubsection{Messungen mit 1,1,2-Trichlortrifluorethan}

1,1,2-Trichlortrifluorethan (TCTFE, $\mathrm{CF}_{2} \mathrm{ClCFCl}_{2}$ ) wurde bereits in IR-PumpUV-Probe-Experimenten an Benzol in kondensierter Phase als schwach wechselwirkendes Lösungsmittel verwendet [44, 97]. Die konsequente Fortführung dieser Studie stellen Messungen in einer gasförmigen Umgebung des gleichen Lösungsmittels dar. Bei einer Meßtemperatur von $100^{\circ} \mathrm{C}$ betrug der Sättigungsdampfdruck des Lösungsmittels ca. 4 bar, was einer reduzierten Dichte der Gasphase von 0.05 entspricht. Experimente über einen weiten Dichtebereich in überkritischem TCTFE wären zwar wünschenswert gewesen, waren mit der bestehenden Apparatur aufgrund der hohen kritischen Temperatur dieser Substanz $\left(T_{\mathrm{c}}=215^{\circ} \mathrm{C}\right)$ jedoch ausgeschlossen.

Ein Resultat der zeitaufgelösten Gasphasenmessungen in TCTFE ist in der unteren Auftragung von Abb. 5.13 gezeigt. Es lassen sich drei Schritte der Dynamik unterscheiden. Einem vom Kreuzkorrelationsartefakt überlagerten schnellen Anstieg auf $0.5 \Delta \mathrm{OD}_{\max }$ schließt sich eine langsamere IVR-Komponente an, aufgrund derer die Signalintensität nach ca. 60 ps ein Maximum durchläuft. Es folgt ein kontinuierlicher Rückgang des Signals auf $0.5 \Delta \mathrm{OD}_{\max }$ nach $250 \mathrm{ps}$. Schon bei dieser geringen Gasphasendichte tritt somit eine signifikante Beschleunigung der intraund intermolekularen Schwingungsrelaxation des Benzols auf.

\subsubsection{Messungen mit $n$-Perfluorpentan}

$n$-Perfluorpentan $\left(\mathrm{C}_{5} \mathrm{~F}_{12}\right)$ wurde als Badgas ausgewählt, da es mit siebzehn Atomen einen sehr effizienten Stoßpartner darstellt, weshalb hiermit die deutlichsten Effekte auf IVR und VET zu erwarten sind. Die kritischen Daten der Substanz $\left(T_{\mathrm{c}}=148^{\circ} \mathrm{C}, p_{\mathrm{c}}=20.5 \mathrm{bar}\right)$ ließen hoffen, daß mit diesem Lösungsmittel auch dichteabhängige Untersuchungen im Stile der $\mathrm{CO}_{2}$-Studie in $[43,96]$ möglich wären. Jedoch zeigten sich auch bei einer Meßtemperatur von $200^{\circ} \mathrm{C}$ in der Zelle für überkritische Lösungen unter Rühren so starke Schlieren, daß kein Signal detektiert werden konnte. Daher mußten die Experimente auf unterkritische Drücke beschränkt werden. Die Signalintensität betrug auch bei einem Benzoldruck von 1 bar nicht mehr als $100 \mu \Delta$ OD, so daß das durch Dichteschwankungen verursachte Rauschen keine optimale Auflösung des Signalverlaufs zuließ. Experimente unter diesen Bedingungen stellen somit die Grenze der Anwendbarkeit der IR-Pump-UV-Probe-Spektroskopie dar. 


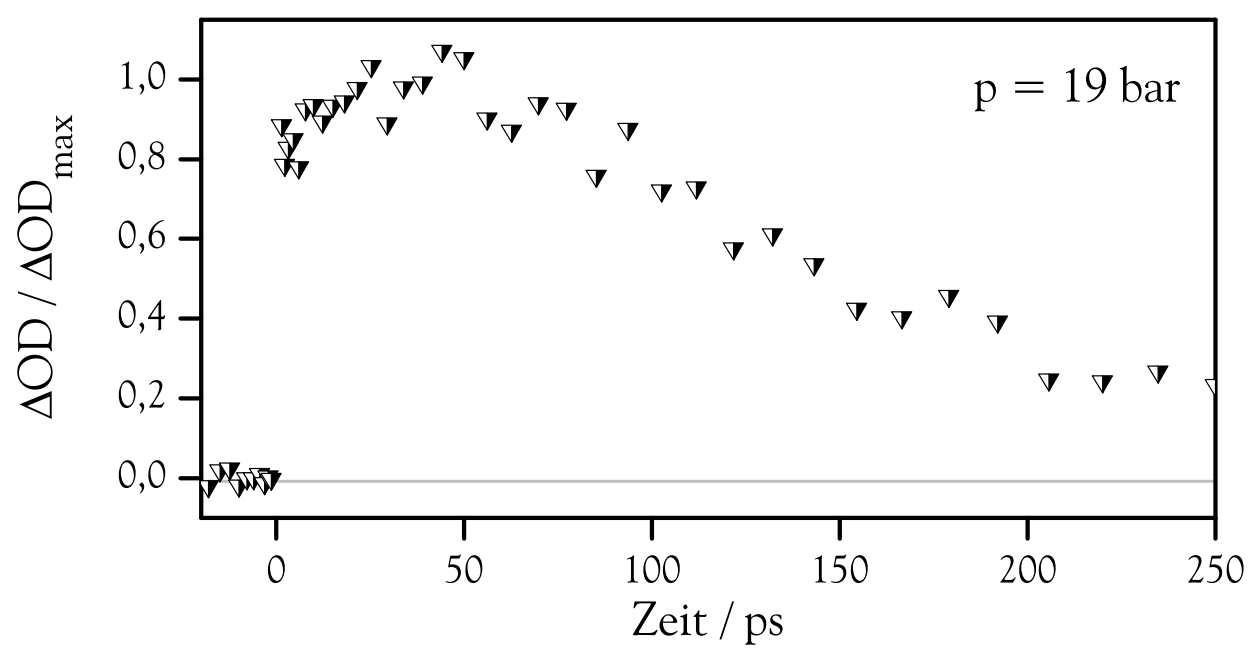

Abbildung 5.15: Transiente Absorption von Benzol in $\mathrm{C}_{5} \mathrm{~F}_{12}$

Abb. 5.15 zeigt beispielhaft das transiente Absorptionsprofil bei dem höchsten realisierbaren Perfluorpentanpartialdruck von 19 bar. Die Meftemperatur betrug $155^{\circ} \mathrm{C}$, woraus sich eine reduzierte $\mathrm{C}_{5} \mathrm{~F}_{12}$-Dichte von 0.42 ergibt. Trotz des suboptimalen Signal-zu-Rausch-Verhältnisses ist eindeutig ein Effekt dieser überkritischen Umgebung auf die beiden Energietransferprozesse des Benzols erkennbar. Eine Analyse des Signalverlaufs zeigt einen rapiden Anstieg direkt nach der Anregung auf $<0.7 \Delta \mathrm{OD}_{\max }$ dem sich die zweite IVR-Komponente anschließt. Das Maximum der transienten Absorption ist nach ca. 50 ps ereicht. Es folgt ein monoexponentieller Abfall der Intensität auf $20 \%$ der maximalen Absorption nach 200 ps.

Diese Ergebnisse zeigen, daß auch in Anwesenheit eines ,großen“ Energieakzeptors wie $n$-Perfluorpentan die im quasi-isolierten Benzolmolekül beobachtete mehrstufige IVR-Dynamik bestehen bleibt.

\subsubsection{Vergleich der Inertgasumgebungen}

Auch in diesem Teil des Kapitels soll ein abschließender Vergleich der Resultate zur Benzoldynamik in verschiedenen molekularen Umgebungen nicht fehlen. Eine 

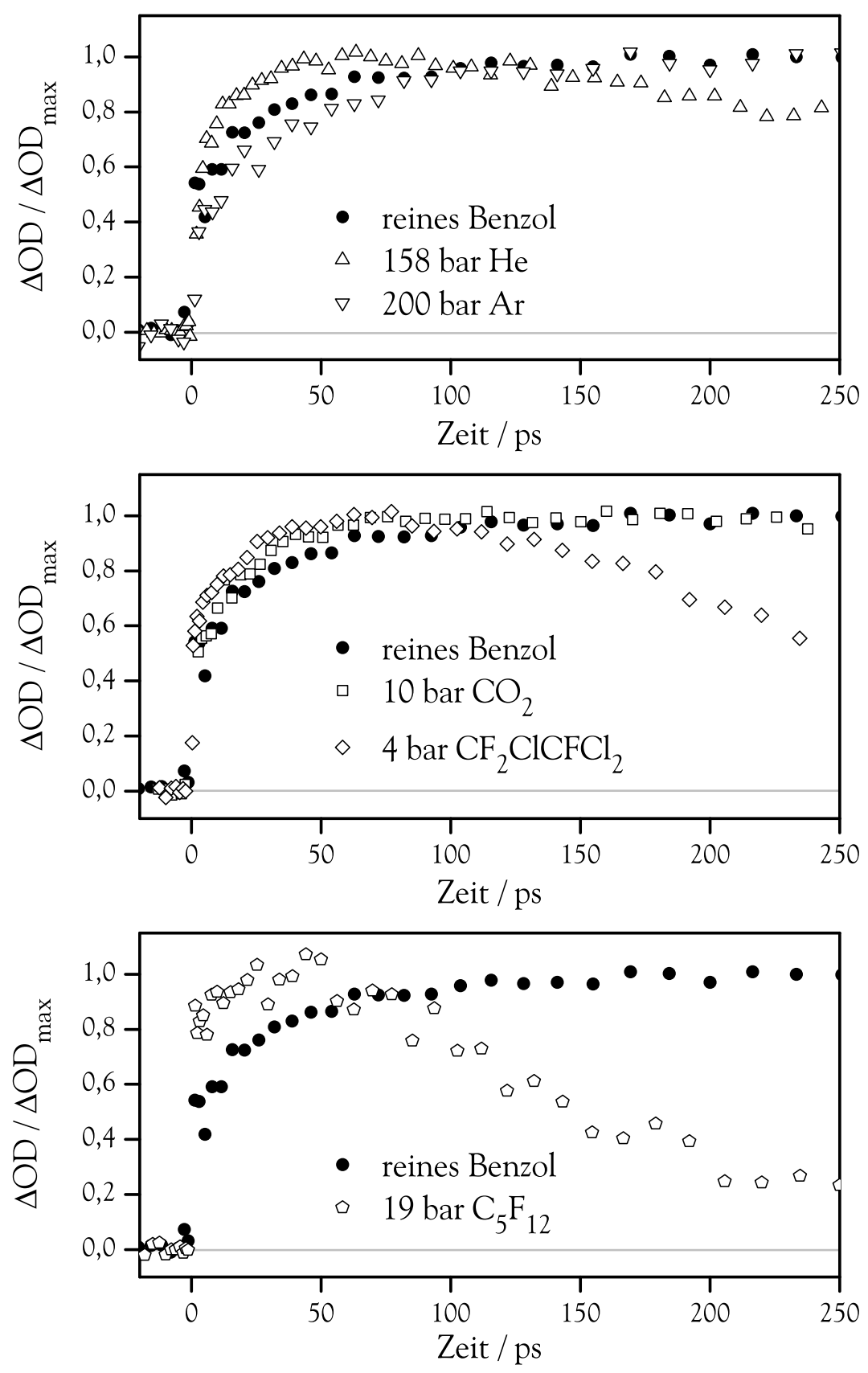

Abbildung 5.16: Vergleich der transienten Absorptionen von Benzol in verschiedenen molekularen Umgebungen 
Auswahl der in den Abschnitten 5.2.1 bis 5.2.5 präsentierten Ergebnisse wurde zu diesem Zweck in Abb. 5.16 zusammengestellt. Im Vergleich mit der transienten Absorption von reinem gasförmigem Benzol lassen sich erste qualitative Aussagen über den Effekt der molekularen Umgebung auf den Schwingungsenergietransfer treffen.

Der erste Graph in Abb.5.16 vergleicht die beiden Edelgase Helium und Argon mit reinem gasförmigem Benzol. Die Häufigkeit von Badgas-Benzol-Stößen beträgt jeweils ca. $1 \mathrm{ps}^{-1}$ (Lennard-Jones-Stoßzahlen). Auf den ersten Blick erscheint die intramolekulare Dynamik in Gegenwart von Helium beschleunigt, in Beisein von Argon hingegen verlangsamt zu werden. Eine genauere Aussage läßt sich aber erst anhand der Zeitkonstanten für die einzelnen IVR- und VETKomponenten formulieren, da bei multiexponentieller Dynamik alle Zeitskalen sowie ihr Verhältnis den Signalanstieg bestimmen.

In Abb. 5.16, Mitte, sind die Ergebnisse der $\mathrm{CO}_{2}$ - und TCTFE-Messungen dem des reinem Benzols gegenübergestellt. Obwohl es sich um vergleichsweise niedrige Badgasdrücke handelt, ist in beiden Fällen eine Beschleunigung der zweiten Komponente der intramolekularen Dynamik festzustellen. Im Gegensatz hierzu tritt nur in Gegenwart des TCTFEs auch eine Zunahme der VET-Geschwindigkeit auf. Unabhängig von der Natur der Gasphasenspezies läuft die intramolekulare Umverteilung weiterhin in mehreren (mind. zwei) Schritten ab, wovon der erste einen gleichbleibenden Beitrag von 0.5 $\Delta \mathrm{OD}_{\max }$ zum Signal leistet.

Die untere Darstellung in Abb. 5.16 veranschaulicht noch einmal den Effekt, den überkritisches $n$-Perfluorpentan auf die Zeitskalen der $2 v_{\mathrm{CH}}$-Relaxation im Benzol hat. Neben einer deutlichen Kontraktion der langsamen IVR-Zeitskala und damit verbundenen Verschiebung des Signalmaximums zu kürzerer Verzögerungszeit ist ein verstärkter Energiefluß aus dem angeregten Molekül in seine Umgebung zu beobachten. 


\subsection{QNS-Simulationen}

Der folgende Abschnitt widmet sich den Ergebnissen, die in Simulationsrechnungen zum intramolekularen Schwingungsenergietransfer mittels des in Abschn. 2.1.4 vorgestellten QNS-Diffusionsmodells erhalten wurden. Mit Hilfe der in Abschn. 2.1.5 eingeführten Methoden zur Reduktion der Rechenzeit durch isotrope Verdünnung des QNS und einer Verbesserung des Simulationsalgorithmus konnten erstmals Systeme mit mehr als sechs Atomen im Bereich experimenteller Anregungsenergien simuliert werden. Aufgrund ihrer vergleichsweise kleinen Zustandsdichte fiel die Wahl auf Benzol und Benzol- $\mathrm{d}_{1}$, um die Gültigkeit des IVR-Diffusionsmodells für aromatische Modellsysteme zu überprüfen. Die Resultate der Pump-Probe-Messungen dieser Arbeit ermöglichen es für beide Moleküle, sowohl für eine CH-Streck-CC-Streckkombinationsanregung als auch für eine $\mathrm{CH}-$ Streckobertonanregung Experiment und Simulation zu vergleichen.

\subsubsection{Generierung der QNS-Signale}

Bevor die einzelnen Simulationsergebnisse vorgestellt werden, muß an dieser Stelle kurz die Signalgenerierung aus den QNS-Rohdaten erläutert werden.

Die Propagation der Besetzung des Startzustandes im QNS liefert zunächst für jeden Schwingungszustand die zeitabhängige Besetzungswahrscheinlichkeit $p_{i}$ (s. S. 21). Hieraus kann durch Rückprojektion der Besetzungswahrscheinlichkeit eines Zustands auf die Normalmoden des Moleküls die zeitabhängige Besetzung der Normalmoden $P(\nu)$ ermittelt werden. Um jedoch ein experimentelles Signal zu rekonstruieren, muß die molekülspezifische Beteiligung verschiedener FCaktiver Normalmoden an der Absorption des Probepulses berücksichtigt werden. Im Fall des Benzols setzt sich das Signal aus den Moden $\nu_{27}$ und $\nu_{29}$ zusammen, wie das hochaufgelöste UV-Absorptionsspektrum von Callomon et al. belegt. Das Intensitätsverhältnis der Banden beträgt hierbei ca. 5:1 [68]. Darüber hinaus existiert eine lineare Abhängigkeit der Absorptionsintensität von der Besetzung der FC-aktiven Moden, da mit dem Probepuls eine Sequenz von Schwingungsbanden abgefragt wird (siehe S. 29). Das simulierte Signal des Benzols wurde daher als $P\left(\nu_{27}\right)+0.2 P\left(\nu_{29}\right)$ berechnet.

Die Zeitauflösung der Pump-Probe-Experimente betrug ca. 1 ps. Zum Zweck eines realistischen Vergleichs wurden daher auch die simulierten Signale mit einer Gaussfunktion entsprechender Breite gefaltet. 


\subsubsection{Kalibration der QNS-Parameter und Simulation experimenteller Absorptions-Zeit-Profile}

Die Kalibration der QNS-Parameter $V_{k}$ (mittleres kubisches Kopplungsmatrixelement), $\lambda$ (Gewichtung des energy gap laws) und $\gamma$ (Gewichtung der Kopplungsordnung) erfolgte anhand des transienten Absorptionsprofils von undeuteriertem Benzol bei einer Pumpwellenlänge von $2140 \mathrm{~nm}$. Der entsprechende Kombinationston als Startzustand im QNS enthält jeweils ein Quant in den Moden $\nu_{4}$ und $\nu_{7}$. Er entspricht somit dem experimentell präparierten ZOBS (s. Abschn. 4.1). Die Breite des Energieintervalls um die Anregungsenergie wurde auf $\Delta E= \pm 5 \mathrm{~cm}^{-1}$ festgelegt. Um eine verläßliche Kalibration zu gewährleisten, wurde die Simulationszeit auf 20 ps ausgedehnt.

Das Ergebnis der Kalibrationssimulationen ist in Abb. 5.17 gezeigt. Mit

$$
V_{k}=4.5 \mathrm{~cm}^{-1}, \quad \gamma=2.5, \quad \lambda=0.25
$$

wurde eine exzellente Reproduktion des transienten Benzolsignals nach Kombinationsanregung erzielt (durchgezogene Linie in Abb. 5.17, oben).

Der zweistufige Signalverlauf innerhalb der ersten 20 ps mitsamt relativer Amplitude und Zeitskala der langsamen IVR-Komponente wird ohne zusätzliche Skalierungsfaktoren der FC-Amplituden korrekt vom QNS-Modell wiedergegeben.

Der Vergleich mit den von Assmann durchgeführten Simulationen an kleinen aliphatischen Molekülen mit Grundton- und/oder Kombinationstonanregung [13] zeigt, daß die Werte von $\lambda$ und $\gamma$ relativ unabhängig vom untersuchten Modellsystem sind. Das mittlere kubische Matrixelement dagegen ist mit $4.5 \mathrm{~cm}^{-1}$ etwas kleiner als bei den Alkylsimulationen.

Bemerkenswerterweise sagt die Simulation für Benzol- $\mathrm{d}_{1}$ unter Verwendung des obigen Parametersatzes bei gleicher Signalzusammensetzung bereits den experimentell beobachteten Trend einer beschleunigten IVR-Dynamik korrekt voraus (gestrichtelte Linie in Abb. 5.17, oben). Den Startzustand der Modellierung bildete ebenfalls die Kombinationsschwingung $1 \nu_{4}+1 \nu_{7}$. Die exakte relative Beteiligung der FC-aktiven Moden an der UV-Absorption durch heiße Banden ist für dieses Molekül bislang leider nicht untersucht worden. Wie in Abschn. 4.2 bereits begründet wurde, darf jedoch angenommen werden, daß in Benzol und 

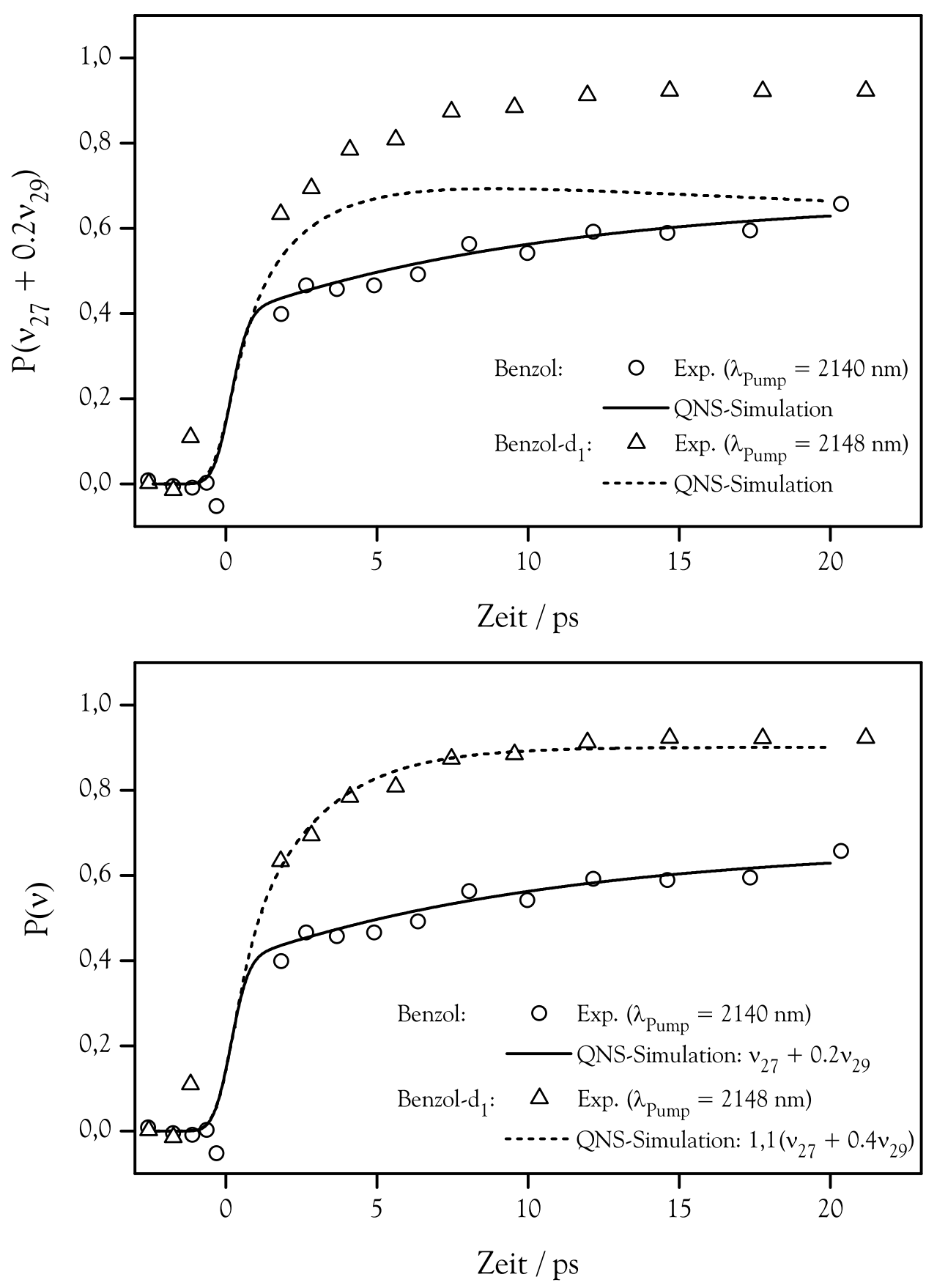

Abbildung 5.17: QNS-Simulation für Kombinationstonanregung $\left(1 \nu_{4}+1 \nu_{7}\right)$ in Benzol und Benzol- $\mathrm{d}_{1}$ 
Kapitel 5 Ergebnisse

Benzol- $\mathrm{d}_{1}$ die gleichen Normalmoden FC-aktiv sind. Gleichzeitig ist eine geringfügige Veränderung der FC-Faktoren dieser Moden durch die Deuterierung nicht auszuschließen. Aus diesem Grund wurde versucht, das experimentelle Signal von Benzol- $\mathrm{d}_{1}$ ausschließlich durch Variation der relativen Beiträge von $\nu_{27}$ und $\nu_{29}$ zu reproduzieren ${ }^{1}$. Mit einer Signalzusammensetzung von 1.1 $\left(\nu_{27}+0.4 \nu_{29}\right)$ konnte eine hervorragende Übereinstimmung mit dem Pump-Probe-Signal erreicht werden (gestrichelte Linie in Abb. 5.17, unten).

Die Ergebnisse der Kalibration - QNS-Parameter und Signalzusammensetzung - bilden die Grundlage für alle weiteren in diesem Kapitel vorgestellen QNSModellrechnungen.

\subsubsection{Vorhersage der IVR-Dynamik nach $2 \mathrm{v}_{\mathrm{CH}}$-Anregung}

Im Experiment wurden für Benzol und Benzol- $\mathrm{d}_{1}$ als ZOBS auch Obertonschwingungen mit ca. $6000 \mathrm{~cm}^{-1}$ Anregungsenergie präpariert. Beide Moleküle besitzen bei diesen Energien mehr als 1500 isoenergetische Schwingungszustände pro $\mathrm{cm}^{-1}$. Eine volldimensionale QNS-Simulation mit dem Experiment nachempfundener Anregungsenergiebreite $\Delta E \geq \pm 20 \mathrm{~cm}^{-1}$ ist zur Zeit noch ausgeschlossen. Es existieren jedoch zwei Möglichkeiten, die Größe des untersuchten QNS zu verringern. Zum einen kann das Energieintervall $\Delta E$ auf ein Minimum von $\pm 5 \mathrm{~cm}^{-1}$ begrenzt werden (ein kleinerer Wert wäre physikalisch nicht sinnvoll). Die Struktur des QNS in unmittelbarer Nähe des ZOBS bleibt hiervon unbeeinträchtigt. Die andere Vorgehensweise besteht darin, eine isotrope Verdünnung des QNS vorzunehmen. So wird die Zustandsdichte pro $N$-dimensionalem Volumenelement verringert, ohne die Isotropie bzw. Anisotropie der Gitterpunktverteilung im Zustandsraum zu verändern.

Unter Beibehaltung der QNS-Parameter aus Abschn. 5.3.2 ist zu erwarten, daß beide Methoden der Zustandsreduktion eine Veränderung der simulierten Dynamik bewirken. Ist der jeweilige Effekt bekannt, kann anhand der Rechnungen in reduzierten Zustandsräumen eine Vorhersage für nicht eingeschränkte Simulationen getroffen werden.

Die Untersuchung der Effekte von Anregungsbreite und QNS-Verdünnung erfolgte für Benzol und Benzol- $\mathrm{d}_{1}$ für die Kombinationstonanregung mit ca. $4500 \mathrm{~cm}^{-1}, 1 \nu_{4}+1 \nu_{7}$, als Startzustand. Die Ergebnisse sind in den Abbildun-

${ }^{1}$ Eine Änderung der an Benzol geeichten QNS-Parameter wurde nicht vorgenommen. 

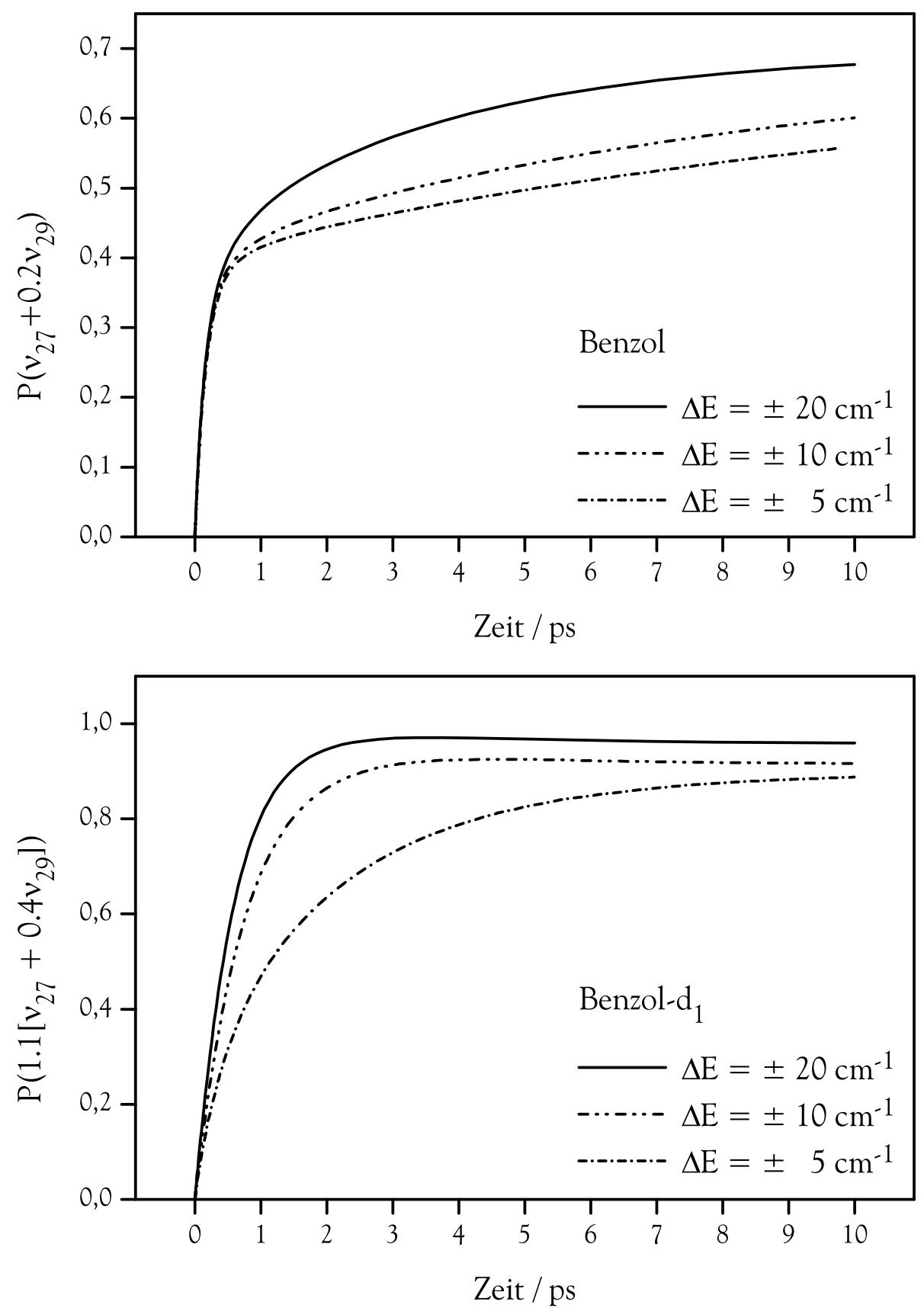

Abbildung 5.18: Effekt der Anregungsenergiebreite $\Delta E$ für Benzol und Benzol-d $\mathrm{d}_{1}$ bei Anregung von $1 \nu_{4}+1 \nu_{7}\left(\right.$ ca. $\left.4500 \mathrm{~cm}^{-1}\right)$ im unverdünnten QNS 

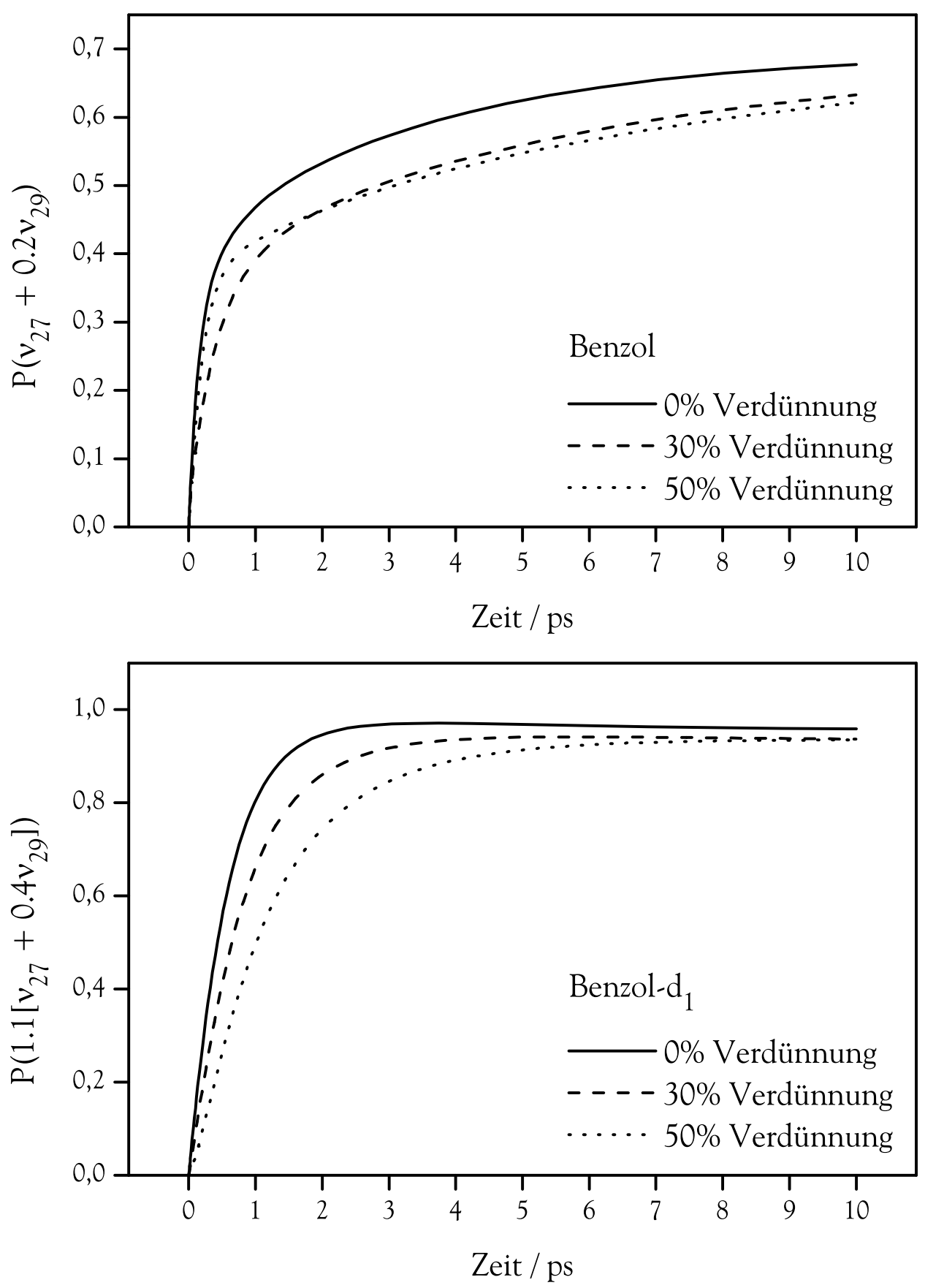

Abbildung 5.19: Effekt der isotropen QNS-Verdünnung für Benzol und Benzol- $\mathrm{d}_{1}$ bei Anregung von $1 \nu_{4}+1 \nu_{7}\left(\right.$ ca. $\left.4500 \mathrm{~cm}^{-1}\right) ; \Delta E= \pm 20 \mathrm{~cm}^{-1}$ 
gen 5.18 und 5.19 zusammengefaßst. Aufgetragen sind die in Abschn. 5.3.2 begründeten Kombinationen der FC-aktiven Moden für 10 ps Simulationszeit. In beiden Fällen unterscheiden sich die Auswirkungen auf die Dynamik von Benzol und Benzol- $\mathrm{d}_{1}$. Für das undeuterierte System ändert sich mit abnehmender Zustandsanzahl lediglich die Amplitude des Signals, während sowohl der charakteristisch sprunghafte frühe Signalanstieg als auch die Steigung des späten Signalanstiegs konstant bleiben. In Deuterobenzol bewirkt die verringerte $\mathrm{Zu}-$ standsdichte hingegen jeweils eine Verlangsamung der Dynamik, wie an der zu späteren Simulationszeiten verschobenen Lage des Maximums zu erkennen ist. Ein quantitativer Vergleich der Signalverläufe ergibt einen Verlangsamungsfaktor von 2.8 für die Verkleinerung des Energieintervalls von $\pm 20 \mathrm{~cm}^{-1}$ auf $\pm 5 \mathrm{~cm}^{-1}$ bzw. 2.2 für eine QNS-Verdünnung von $50 \%$. Eine lineare Abhängigkeit zwischen simulierter IVR-Zeitskala und der Zustandsdichte im QNS liegt nicht vor.

In den QNS-Simulationen für die $2 v_{\mathrm{CH} \text {-streck }}$-Anregung wurde in Einklang mit den IR-Auswahlregeln der Punktgruppen $\mathrm{D}_{6 \mathrm{~h}}$ und $\mathrm{C}_{2 \mathrm{v}}$ (s. Abschn. 4.1 und 4.2) die Kombinationsmode $1 \nu_{4}+1 \nu_{5}$ als Startzustand für Benzol und die Obertonschwingung $2 \nu_{5}$ als Startzustand für Benzol- $\mathrm{d}_{1}$ gewählt. In diesem Fall liegen bei Deuterobenzol selbst mit einem minimalen Energieintervall von $\pm 5 \mathrm{~cm}^{-1}$ noch $2.4 \cdot 10^{4}$ Zustände im QNS, weswegen der Zustandsraum darüber hinaus um $50 \%$ verdünnt werden mußte. Unter den gleichen Bedingungen wurden desweiteren für beide Systeme nochmals die Kombinationsanregungen $1 \nu_{4}+1 \nu_{7}$ simuliert, um einen eindeutigen Vergleich zwischen Obertons- und Kombinationstonsrelaxation zu ermöglichen.

Abb. 5.20 enthält die Resultate der Rechnungen in den reduzierten Zustandsräumen. Das auffälligste Merkmal der simulierten Pump-Probe-Signale ist, daß sowohl für Benzol als auch für das monodeuterierte Derivat eine sehr ähnliche Dynamik bei den verschiedenen Anregungsenergien vorhergesagt wird. Dies stimmt mit den experimentellen Beobachtungen hervorragend überein, wie der Vergleich mit Abb. 5.2 bzw. 5.7 zeigt. Bei näherer Betrachtung erscheint die Relaxation nach Kombinationstonanregung in beiden Molekülen marginal langsamer als nach der entsprechenden Obertonanregung fortzuschreiten. Die in Abschn. 6.2.1 ermittelten absoluten IVR-Zeitkonstanten belegen, daß dies dem experimentellen Befund entspricht.

Ferner läßt sich selbst im stark reduzierten QNS die Tendenz einer Beschleunigung des IVR-Prozesses durch die Monodeuterierung reproduzieren. Unter Be- 


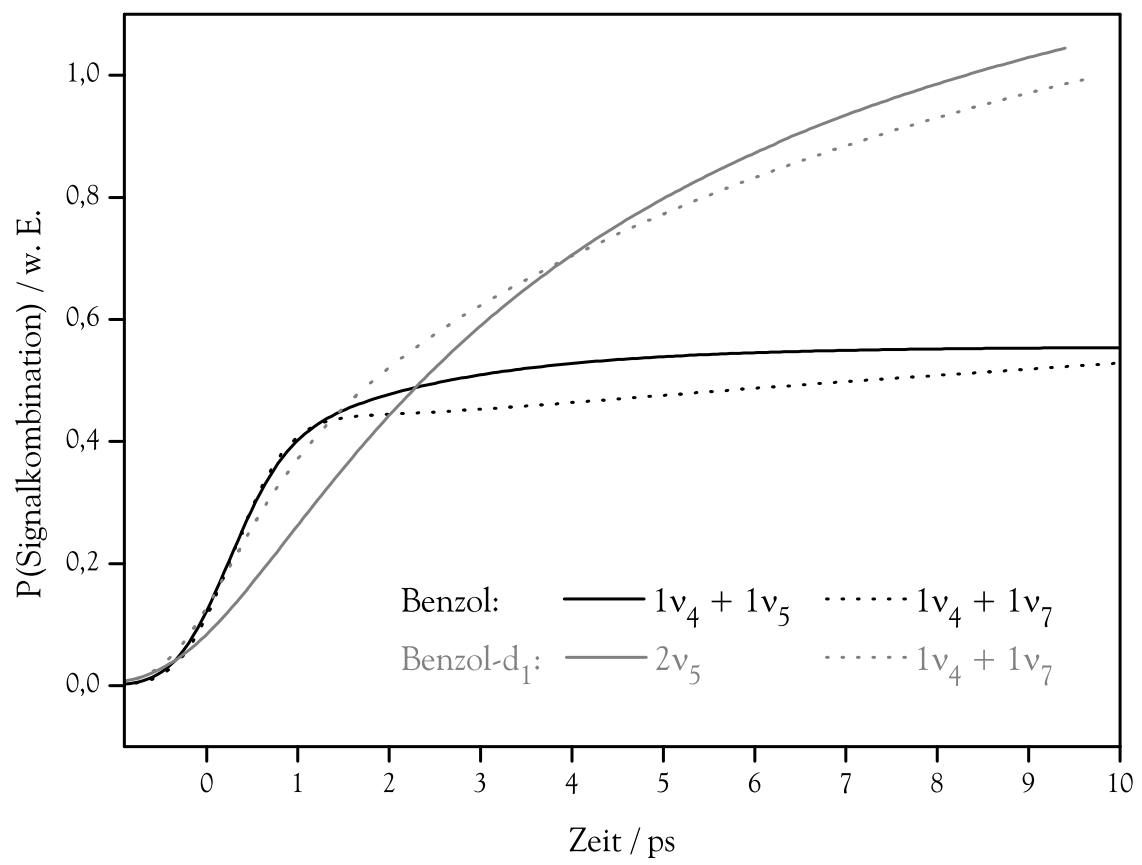

Abbildung 5.20: QNS-Simulation für Benzol und Benzol-d $\mathrm{d}_{1}$ bei 6000 bzw. $4500 \mathrm{~cm}^{-1}$ Anregungsenergie mit $\Delta E= \pm 5 \mathrm{~cm}^{-1}$ und $50 \%$ QNS-Verdünnung

rücksichtigung der IVR-Verlangsamung für Benzol-d $\mathrm{d}_{1}$ um einen Faktor ca. 5 durch Energieintervall und QNS-Verdünnung wäre in einer uneingeschränkten Simulation eine noch stärkere Ausprägung dieses Trends und damit eine bessere Wiedergabe der experimentellen Ergebnisse zu erwarten.

\subsubsection{Vorhersage der IVR-Dynamik nach $1 \mathrm{v}_{\mathrm{CH}}$-Anregung}

Im vorhergehenden Abschnitt wurde gezeigt, daßs sich Simulationen mit dem QNS-Diffusionsmodell eignen, um zeitaufgelöste IVR-Transienten mit verschiedenen Anregungsenergien zu reproduzieren. Dies konnte anhand der Pump-ProbeDaten dieser Arbeit für die Oberton- bzw. Kombinationstonanregung von sowohl $\mathrm{C}_{6} \mathrm{H}_{6}$ als auch $\mathrm{C}_{6} \mathrm{H}_{5} \mathrm{D}$ in der Gasphase verifiziert werden. 


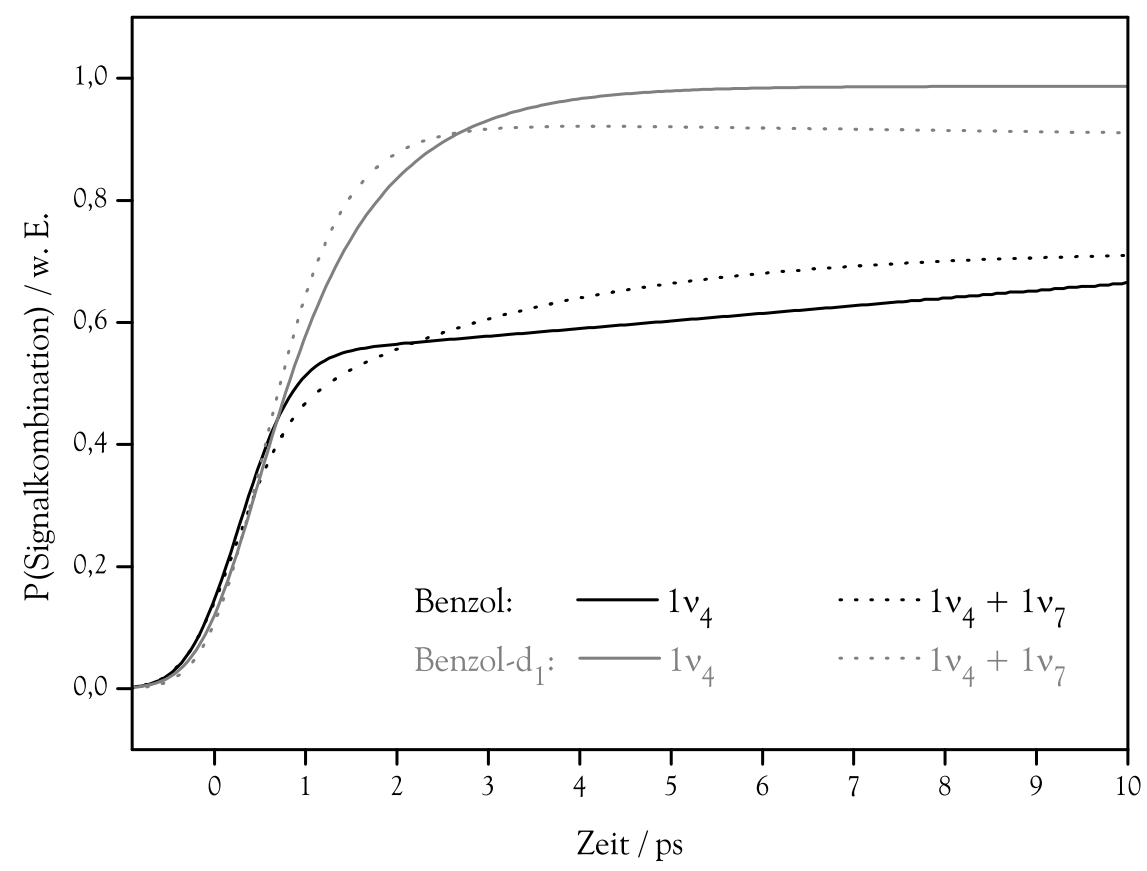

Abbildung 5.21: QNS-Simulation für Benzol und Benzol-d $\mathrm{d}_{1}$ bei 3000 bzw. $4500 \mathrm{~cm}^{-1}$ Anregungsenergie mit $\Delta E= \pm 20 \mathrm{~cm}^{-1}$ und $0 \%$ QNS-Verdünnung

In Hinblick auf die Validierung der QNS-Annahmen wäre es höchst interessant, die Relaxation einer Obertonschwingung mit der entsprechenden Fundamentalanregung zu vergleichen, da es sich in beiden Fällen um edge states derselben Mode handelt. Eine derartige Gegenüberstellung unter Verwendung einheitlicher QNS-Bedingungen ( $\Delta E$, Verdünnung) ist bei Benzol aufgrund der stark unterschiedlichen Zustandsdichte bei 3000 bzw. $6000 \mathrm{~cm}^{-1}$ jedoch mit den zurzeit verfügbaren Rechenkapazitäten ausgeschlossen. Der Vergleich mit einer Kombinationsanregung von CH- und CC-Streckmoden ist hingegen durchaus möglich. Es wurden daher an Benzol und Benzol- $\mathrm{d}_{1}$ Simulationen mit $\Delta E= \pm 20 \mathrm{~cm}^{-1}$ in unverdünntem QNS für die Startzustände $1 \nu_{4}$ und $1 \nu_{4}+1 \nu_{7}$ ausgeführt. Die Werte der QNS-Parameter $V_{k}, \gamma$ und $\lambda$ betrugen weiterhin $4.5 \mathrm{~cm}^{-1}, 2.5$ bzw. 0.25 . In Abb. 5.21 sind die berechneten Signale dargestellt. Wiederum zeichnen sich beide Signale desselben Moleküls durch einen sehr ähnlichen Verlauf aus (schwarze bzw. 
graue Kurven). Die Anregungsenergie erreicht bei $1 \nu_{4}+1 \nu_{7}$-Anregung jeweils etwas schneller die FC-aktiven Moden, was durch die größere Gitterpunktdichte in diesem Teil des QNS zu erklären ist.

Die Dynamik des deuterierten Derivates ist auch bei $3000 \mathrm{~cm}^{-1}$ Anregungsenergie gegenüber Benzol beschleunigt (durchgezogene Kurven). Hier erreicht das Signal innerhalb der Simulationszeit nach ca. 6 ps ein konstantes Niveau, während bei Benzol auch noch 10 ps noch immer eine positive Steigung vorhanden ist. Somit sagt das IVR-Diffusionsmodell unabhängig von der Art der CHStreckschwingungsanregung - als Fundamentale, Kombinationsmode oder Oberton - generell eine Beschleunigung der intramolekularen Dynamik durch die Monodeuterierung voraus.

\section{Vergleich mit experimentellen Daten}

Bislang existieren keine zeitaufgelösten IVR-Studien zur Grundtonrelaxation von $\mathrm{C}_{6} \mathrm{H}_{6}$ oder $\mathrm{C}_{6} \mathrm{H}_{5} \mathrm{D}$ in der Gasphase, anhand derer die Vorhersagekraft der QNSSimulationen überprüft werden könnte. Stewart und McDonald nahmen zwar frequenzaufgelöste Gasphasen-Fluoreszenzmessungen an Benzol vor, fanden nach CH-Streckanregung mit ca. $3000 \mathrm{~cm}^{-1}$ jedoch keine Hinweise auf intramolekulare Energieumverteilung [98]. Die Autoren führten dies auf die geringe Zustandsdichte des Systems zurück.

Die einzige zeitaufgelöste Studie zur CH-Streckschwingungsrelaxation in Aromaten wurde Dlott und Mitarbeitern an Benzol in der kondensierten Phase durchgeführt [99]. Als Lösungsmittel wählten sie $\mathrm{CCl}_{4}$, das als schwach wechselwirkend (unpolar, aprotisch) klassifiziert werden kann. Mit Hilfe der Anti-StokesRaman-Spektroskopie beobachteten sie nach Anregung von $\nu_{2}\left(3064 \mathrm{~cm}^{-1}\right)$ die zeitabhängige Populationsänderung der Moden $\nu_{4}\left(3057 \mathrm{~cm}^{-1}\right), \nu_{19}\left(993 \mathrm{~cm}^{-1}\right)$ und $\nu_{27}\left(608 \mathrm{~cm}^{-1}\right)$. Die Zeitauflösung betrug ca. 1 ps bei einer spektralen Anregungsbreite von ca. $35 \mathrm{~cm}^{-1}$. Die gleichen Bedingungen wurden IVR-Simulationen zugrundegelegt, deren QNS-Parameter weiterhin die Gasphasenwerte besaßen. Anhand der Abb. 5.22 lassen sich die Resultate wie folgt zusammenfassen.

- Der konsekutive Energiefluß durch die drei Moden in der Reihenfolge $\nu_{4} \rightarrow$ $\nu_{19} \rightarrow \nu_{27}$ wird in der Simulation korrekt wiedergegeben, ebenso wie der transiente Charakter von $\nu_{4}$. 
- Im Experiment zeigen alle drei Moden eine instantane, von der Zeitauflösung begrenzte Komponente des Signalanstiegs. Dieser Trend spiegelt sich in den modellierten Signalen ebenfalls korrekt wieder.

- Die Simulation liefert eine deutlich schnellere Abklingdynamik der hochfrequenten Mode $\nu_{4}$ als das Experiment. Gleichzeitig ist in der Modellierung auch nach 30 ps ein Restenergiegehalt in dieser Schwingung zu verzeichnen.

- Die zweite, langsamere Komponente der Populationszunahme in $\nu_{19}$ sowie der Signalrückgang dieser Mode fehlen in der berechneten Dynamik.

- Für $\nu_{27}$ stimmen Experiment und Simulation sehr gut überein.

Ein Vergleich der experimentellen und berechneten Modenpopulationen gestattet es, eine qualtitative Abschätzung des Lösungsmitteleinflusses auf den IVRProzess vorzunehmen.

Unter der Annahme, daß die Wechselwirkung zwischen Solvat- und Solvensmolekül durch Stöße vermittelt wird, kann die exakte Reproduktion der sub-psIVR-Komponente in der Gasphasensimulation dadurch erklärt werden, daß der entsprechende IVR-Schritt in der Zeit zwischen zwei Stößen bereits vollzogen ist und daher nur einen geringen Lösungsmitteleinfluß spürt.

Der im Vergleich zur Simulation beschleunigte Signalrückgang in der Mode $\nu_{19}$ läßt sich auf intermolekularen Schwingungsenergietransfer an die Lösungsmittelumgebung zurückführen, welcher im QNS-Modell nicht berücksichtigt wird. Des weiteren ist es wahrscheinlich, daß die Unterschiede zwischen Theorie und Experiment in der relativ kleinen Zustandszahl im QNS $(<500)$ begründet sind. Hierdurch werden eventuelle Anisotropien der Gitterpunktverteilung im QNS stärker gewichtet.

Abschließend ist festzuhalten, daß eine qualitative Reproduktion der konsekutiven Bevölkerung der drei Moden mit dem Gasphasenmodell eines sequentiellen Energieflusses gelungen ist. Dies deutet darauf hin, daß die IVR-Mechanismen des isolierten Moleküls in der schwach wechselwirkenden Lösungsmittelumgebung prinzipiell erhalten bleiben.

An diesem Punkt ist eine Gegenüberstellung des Ergebnisses von Dlott et al. mit den IR-Pump-UV-Probe-Messungen in Ref. [94] zur Oberton- und Kombinationstonrelaxation von Benzol in Lösung angebracht. Da $\nu_{27}$ den Hauptteil der Franck-Condon-Aktivität des Moleküls trägt, läßt sich die IVR-Zeitskala aus 


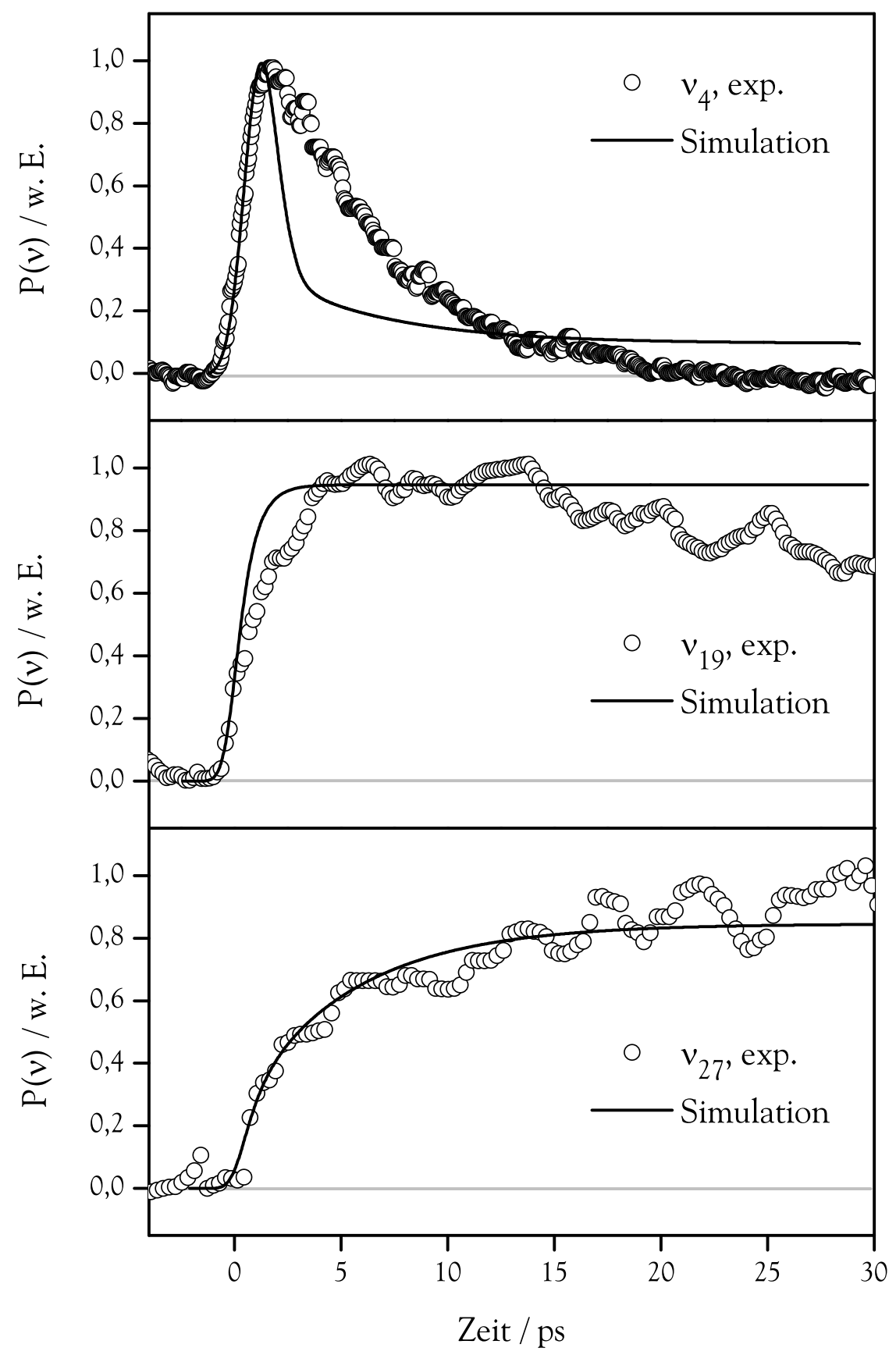

Abbildung 5.22: QNS-Simulation für Benzol nach $1 \nu_{2}$-Anregung, Vergleich mit Lösungsexperiment von Dlott et al. [99] 
den transienten Absorptionsexperimenten in [94] gut mit der von Dlott gemessenen Dynamik vergleichen. In Lösung findet die intramolekulare Umverteilung der Anregungsenergie sowohl nach $2 \nu_{\mathrm{CH} \text {-streck }}-$ als auch nach $\left(\nu_{\mathrm{CH}-\text { streck }}+\nu_{\mathrm{CC} \text {-streck }}\right)$ Anregung mit 6000 bzw. $4500 \mathrm{~cm}^{-1}$ auf einer effektiven 2 ps-Zeitskala statt. Sie erfolgte damit sehr viel schneller als nach $1 \nu_{\mathrm{CH}-\text { streck}}$-Anregung. Genaugenommen erinnert die Dynamik in Abb. 5.22, unten, eher an typische Gasphasenzeitskalen. Dies spricht dafür, daß die bekanntermaßen [98] äußerst ineffiziente Kopplung des CH-Streck-Grundtons an die wenigen isoenergetischen Hintergrundzustände selbst durch Wechselwirkung mit dem Lösungsmittel nicht signifikant verbesser werden kann. Diese Vermutung wird durch die korrekte Vorhersage der $\nu_{27}$-Dynamik in $\mathrm{CCl}_{4}$ mittels des Gasphasen-IVR-QNS-Modells unterstützt. 



\section{Kapitel 6}

\section{Auswertung}

Ziel der Pump-Probe-Experimente ist es, aus den gemessenen transienten Absorptionen Informationen über den intramolekularen sowie den intermolekularen Energietransfer zu erhalten. Daher wird in diesem Kapitel zunächst der Zusammenhang zwischen der zeitabhängigen Absorptionsänderung und der Energieumverteilung verdeutlicht. Anschließend wird das kinetische Modell zur Simulation der experimentellen Daten vorgestellt. Aus der Anpassung der simulierten an die gemessenen transienten Absorptionen ergeben sich die molekülspezifischen IVRund VET-Zeitkonstanten, welche die Grundlage der Diskussion des Energietransfers in Kap. 7 bilden werden.

\subsection{Grundlagen der Auswertung}

\subsubsection{Interpretation der Pump-Probe-Signale}

Das Konzept der IR-Pump-UV-Probe-Spektroskopie basiert auf der Anregung und Abfrage unterschiedlicher, indirekt gekoppelter Schwingungsfreiheitsgrade desselben Moleküls. Entsprechend der in Abschn. 2.1 eingeführten Terminologie bildet die Superposition der vom Pumppuls angeregten hellen Zustände hierbei den sog. ZOBS (zeroth order bright state) des Systems. Zur Absorption des Probepulses tragen hingegen Hintergrundzustände mit Beteiligung FC-aktiver Moden bei, welche nicht direkt vom Anregungspuls populiert werden. Eine Zuordnung der mit den Laserpulsen wechselwirkenden Normalmoden ist anhand einer Analyse der Absorptions- bzw. Fluoreszenzemissionsspektren des jeweiligen Modellsystems möglich (s. Kap. 4). 
Eine genauere Betrachtung des Lokalisierungsgrades der Anregungsenergie im Verlauf eines typischen Pump-Probe-Experiments liefert den Ansatz für die Interpretation der transienten Absorptionssignale.

Zum Zeitpunkt $t=0$ deponiert ein fs-IR-Puls die Anregungsenergie im ZOBS des Moleküls. Die Überschußenergie beträgt bei Pulsen mit ca. $1600 \mathrm{~nm}$ Wellenlänge ca. $6000 \mathrm{~cm}^{-1}$, bei $2140 \mathrm{~nm}$-Pulsen dagegen ca. $4500 \mathrm{~cm}^{-1}$. Obwohl aufgrund der hohen Zustandsdichte der aromatischen Modellsysteme bei diesen Energien keine selektive Anregung eines einzelnen Schwingungsfreiheitsgrades erreicht wird, kann trotzdem von einer stark lokalisierten Anregung zu Beginn des Experiments gesprochen werden, weil die Anzahl zum ZOBS beitragender Zustände im Vergleich zur Anzahl der isoenergetischen dunklen Zustände äußerst gering ausfällt.

Da es sich bei dem ZOBS um keinen Eigenzustand des molekularen Hamiltonoperators handelt, wird das System eine Umverteilung der lokalisierten Schwingungsenergie $E_{\text {lok }}$ anstreben. Diese Equilibrierung kann sowohl durch intra- als auch durch intermolekulare Kopplungen vermittelt werden. Wie in Abschn. 2.2 bereits dargelegt, findet die Redistribution aus hochfrequenten Schwingungsfreiheitsgraden, zu denen die hier präparierten CH-Streckschwingungsobertöne zählen, bevorzugt über intramolekulare Kanäle statt, da

- generell eine exponentielle Abhängigkeit des intermolekularen Energietransfers von der Ordnung der Kopplung und somit vom Frequenzunterschied des Energiedonors und -akzeptors vorliegt;

- in einer verdünnten molekularen Umgebung, wie sie in den Gasphasenexperimenten gegeben ist, die Frequenz bimolekularer Stöße, in denen Schwingungsenergie übertragen werden kann, niedrig im Vergleich zur intrinsischen Zeitskala des IVR-Prozess ist.

Es existieren somit gleichzeitig eine qualitative und eine quantitative Einschränkung der intermolekularen Relaxationsmöglichkeiten. Bevorzugte Kopplungspartner des ZOBS werden aus diesem Grund die intramolekular zugänglichen Hintergrundzustände sein. Die Energieumverteilung auf alle nicht-angeregten Schwingungsfreiheitsgrade des Modellsystems entspricht dann der Ausbildung eines mikrokanonischen Ensembles. Mit den Bezeichnungen $E_{\text {lok }}$ für die lokalisierte Anregungsenergie und $E_{\text {mik }}$ für die mikrokanonisch umverteilte Schwingungsenergie 
kann diese komplexe Equilibrierung stark vereinfachend als globaler Prozeß erster Ordnung beschrieben werden:

$$
E_{\mathrm{lok}} \stackrel{k_{\mathrm{IVR}}}{\longrightarrow} E_{\text {mik }}
$$

Die Größe $E_{\text {mik }}$ repräsentiert hierbei den Zustand des Systems nach abgeschlossener IVR-Dynamik, wenn die Anregungsenergie auf sämtliche isoenergetischen Zustände umverteilt ist. Unter Berücksichtigung des in Abschn. 2.1.2 vorgestellten Stufenmodells sequentiellen Energieflusses ist eine explizite Formulierung der IVR-Kinetik als Folgereaktion gemäß

$$
E_{\mathrm{lok}} \stackrel{k_{\mathrm{IVR}_{1}}}{\longrightarrow} E_{\mathrm{red}_{1}} \stackrel{k_{\mathrm{IVR}_{2}}}{\longrightarrow} E_{\mathrm{red}_{2}} \ldots \stackrel{k_{\mathrm{IVR}_{n}}}{\longrightarrow} E_{\mathrm{mik}}
$$

allerdings angemessener. In diesem Fall bezeichnet $E_{\text {red }_{i}}$ den Zustand des Systems nach abgeschlossener Umverteilung der Energie auf den $i$-ten tier. Die globale IVR-Geschwindigkeit ist dann als Summe der $i$ Einzelschritte aufzufassen.

Wie äußert sich nun die intramolekulare Energieumverteilung in den transienten Absorptionsprofilen des Experiments? In den Kapiteln 4.1 bis 4.5 wurde ausführlich dargelegt, daß die Absorption eines UV-Pulses auf der roten Flanke des ersten elektronischen Übergangs durch heiße Banden FC-aktiver Normalmoden verursacht wird. Somit stellt die Absorption eines entsprechenden Pulses ein direktes Maß für die Population dieser Schwingungsfreiheitsgrade dar. Es hat sich außerdem für alle Modellsysteme bestätigt, daß der ZOBS keine signifikanten Anteile der FC-Moden trägt. Eine instantane Besetzung dieser Schwingungen durch den IR-Anregungspuls kann folglich ausgeschlossen werden. Stattdessen können diese Freiheitsgrade im Zuge der intramolekularen Umverteilung populiert werden. Unter der Voraussetzung, daß die FC-aktiven Moden vorwiegend an Zuständen späterer Umverteilungsstufen mitwirken, stellt ihre Bevölkerung - und somit die Zunahme der UV-Absorption - ein Maß für den Fortschritt des gesamten IVR-Prozesses, d.h. der Ausbildung von $E_{\text {mik }}$, dar. Da im Falle der Aromaten die FC-aktiven Moden generell zu den niederfrequentesten Molekülschwingungen zählen, welche aufgrund des großen Abstands der Energieniveaus bei niedriger Ordnung schlecht an den hochfrequenten ZOBS koppeln, ist obige Voraussetzung erfüllt.

Durch die Umverteilung erreicht die Schwingungsenergie zunehmend niederfrequente Moden, die effektiver an das Energiebad koppeln. Auch haben mit 
Kapitel 6 Auswertung

zunehmender Verzögerungszeit zwischen Pump- und Probepuls mehr Stöße mit „kalten“ Badgasteilchen stattgefunden. Der intramolekularen Equilibrierung folgt somit der verstärkte intermolekulare Transfer der Anregungsenergie in die molekulare Umgebung. Dies bewirkt die sukzessive Abkühlung des mikrokanonischen Ensembles zu einer letztlich thermischen Verteilung der Energie $E_{\text {eq }}$ über alle Freiheitsgrade des Gesamtsystems. Die damit einhergehende Entvölkerung der FC-aktiven Moden resultiert dann in einem Rückgang der Absorption des UV-Probepulses. Im einfachsten Fall läßt sich die intermolekulare Equilibrierung als Prozeß erster Ordnung gemäß

$$
E_{\mathrm{mik}} \stackrel{k_{\mathrm{VET}}}{\longrightarrow} E_{\mathrm{eq}}
$$

auffassen.

Für transiente Absorptionsprofile aus Messungen in sehr dichten molekularen Umgebungen erlaubt diese Interpretation des VET-verursachten Signalrückgangs die direkte Bestimmung der intermolekularen Wechselwirkungszeitskalen. In Abwesenheit eines Inertgases gestaltet sich diese Analyse hingegen problematisch. Durch Selbststöße werden hier in V-V-Transferschritten die FC-aktiven Moden kalter aromatischer Moleküle bevölkert, die sich in ihrem Absorptionsverhalten von dem ursprünglich angeregten Teilchen nicht unterscheiden lassen. Da so die Anregungsenergie erst durch Diffusion der Akzeptormoleküle aus dem Probevolumen (ns-Zeitskala) bzw. durch V-R- und V-T-Transfer für den UV-Probepuls „unsichtbar" wird, findet die Signalabnahme auf einer künstlich verlängerten Zeitskala statt. Bei linearer Abhängigkeit der optischen Dichte von der inneren Energie wäre im Falle reinen V-V-Transfers in der experimentell zugänglichen Zeitspanne von einigen hundert Pikosekunden gar kein Absorptionsrückgang zu erwarten.

Aus Gasphasen-IR-Pump-UV-Probe-Experimenten bestimmte Zeitskalen intermolekularer Dynamik stellen somit keine echten VET-Zeiten dar. Dennoch sind sie im kinetischen Modell, welches im Abschnitt 6.1.2 vorgestellt wird, in Analogie zu früheren Arbeiten (z. B. [44]) als VET-Zeitskalen inkorporiert, um eine Simulation des gesamten Signalverlaufs zu ermöglichen. Eine alternative Modellierung könnte einen zusätzlichen exponentiellen Term beinhalten, welcher das Aufheizen der Umgebung mit der gleichen Geschwindigkeit wie die Abkühlung des angeregten Teilchens beschreibt. Für eine eindeutige Anpassung der damit verbundenen zusätzlichen Parameter könnten aus $\langle\Delta E\rangle$-Werten und Lennard- 
Jones-Stoßzahlen berechnete VET-Zeiten benutzt werden. Allerdings sind nur für einige der in dieser Arbeit untersuchten Aromat-Stoßgas-Kombinationen $\langle\Delta E\rangle$ Werte dokumentiert. Zudem sind einige dieser Werte zu fehlerbehaftet, um eine verläßliche Vorhersage des VET zu gestatten. Aus diesen Gründen wurde von einer Modellierung inklusive Aufheizterm abgesehen.

\subsubsection{Kinetische Modellierung}

Die ersten kinetischen Modelle zur Simulation von IR-Pump-UV-ProbeMessungen wurden formuliert, um experimentelle Daten aus der kondensierten Phase anzupassen $[13,44]$. Im einfachsten Fall einer Folgereaktion entsprechend

$$
E_{\mathrm{lok}} \stackrel{k_{\mathrm{IVR}}}{\longrightarrow} E_{\mathrm{red}} \stackrel{k_{\mathrm{VET}}}{\longrightarrow} E_{\mathrm{eq}}
$$

ergab sich aus der Lösung der zugehörigen Differentialgleichung eine Anpassungsfunktion mit monoexponentiellem Anstieg und anschließendem monoexponentiell abfallendem Verlauf.

Im Fall der transienten Absorptionen aus den Gasphasenmessungen dieser Arbeit (s. Kap. 5) ist sofort ersichtlich, daß eine lediglich monoexponentielle Beschreibung der intramolekularen Dynamik völlig unzulänglich wäre. In allen Absorptionsprofilen existieren mindestens zwei Komponenten des Signalanstiegs, was mit der Vorstellung eines sequentiellen intramolekularen Energieflusses gut zu vereinbaren ist. Die VET-bedingte Signalabnahme kann hingegen mit einer monoexponentiellen Form modelliert werden.

Aus diesen Überlegungen ergibt sich eine phänomenologische Anpassungsfunktion $F(t)$ mit drei exponentiellen Termen.

$$
F(t)=\exp \left(\frac{-t}{\tau_{\mathrm{VET}}}\right)-\left[A_{\text {inst }} \exp \left(\frac{-t}{\tau_{\mathrm{IVR}_{1}}}\right)+\left(1-A_{\text {inst }}\right) \exp \left(\frac{-t}{\tau_{\mathrm{IVR}_{2}}}\right)\right]
$$

Es wird hierbei der einfachste Fall eines konsekutiven IVR-Prozesses mit zwei Schritten angenommen. Die Zeitkonstanten $\tau_{\mathrm{IVR}_{1}}$ und $\tau_{\mathrm{IVR}_{2}}$ bestimmen die Dynamik der Absorptionszunahmen, während die Zeitkonstante $\tau_{\text {VET }}$ die Abnahme der Absorption quantifiziert. Der Zusammenhang zu den Geschwindigkeitskonstanten der Gleichungen (6.2) und (6.3) wird über die Relation $\tau=1 / k$ hergestellt. Der Wert von $\tau_{\mathrm{IVR}_{1}}$ ist durch die Zeitauflösung des Experiments begrenzt (und festgelegt). 
Die relativen Amplituden $A_{\text {inst }}$ bzw. $\left(1-A_{\text {inst }}\right)$ legen den Anteil des ersten, ,instantanen“, und des zweiten IVR-Schrittes an der gesamten intramolekularen Dynamik fest. Da die experimentellen und simulierten Daten standardmäßig auf $\Delta \mathrm{OD}_{\max }$ normiert werden, müssen im Gegensatz zu früheren Formulierungen dieses Modells [94] außer $A_{\text {inst }}$ keine weiteren Amplitudenkoeffizienten berücksichtigt werden.

\section{Vorgehensweise zur Signalanpassung}

Die Simulation der experimentellen Daten erfolgte mit dem Programm MathCad (Mathsoft Engineering \& Education, V. 12.0).

Die Zeitkonstanten $\tau$ und relative Amplitude $A_{\text {inst }}$ wurden zunächst anhand einer Abschätzung festgelegt und in die Anpassungsfunktion $F(t)$ eingegeben. Aus $F$ wurde die zeitabhängige innere Energie des Moleküls $E(t)$ gemäß

$$
E(t)=E_{\mathrm{Pump}} \cdot F(t)+E_{\mathrm{T}}
$$

berechnet. Die hierfür benötigte Pumpenergie $E_{\text {Pump }}$ ergab sich direkt aus der Wellenlänge der verwendeten Anregungspulse. Einen zweiten, zeitunabhängigen Beitrag zur inneren Energie leistet die thermische Energie $E_{\mathrm{T}}$ des Moleküls bei der jeweiligen Meßtemperatur, welche anhand von Gl. (4.1) bestimmt werden konnte.

Für eine Erläuterung der Kalibration der transienten Absorption, welche einen fundamentalen Aspekt der Auswertung darstellt, sei der Leser auf die Einleitung zu Kap. 4 verwiesen. Die Eichkurven zur Energieabhängigkeit der optischen Dichte sind in den Abschnitten 4.1-4.5 ermittelt worden. Es handelt sich um Polynome 3. Grades, deren Koeffizienten in Anhang A.1 aufgeführt sind. Durch Einsetzen von Gl. (6.6) in diese Eichpolynome konnte die zeitabhängige Änderung der optischen Dichte berechnet werden.

$$
\Delta \mathrm{OD}(t)=\sum_{n=0}^{3} a_{n}[E(t)]^{n}
$$

Die Zeitauflösung des Experiments ist in der Gerätefunktion

$$
g(t)=\exp \left[-4 \ln 2\left(\frac{t-t_{0}}{w_{\mathrm{G}}}\right)^{2}\right]
$$


berücksichtigt. Es wurde eine gaußförmige Kreuzkorrelation mit einer der Zeitauflösung entsprechenden Halbwertsbreite $w_{\mathrm{G}}$ und Maximum bei $t_{0}$ angenommen. Die endgültige Simulationsfunktion $S(t)$ resultierte aus einer Faltung der optischen Dichte mit dieser Gerätefunktion.

$$
S(t)=\Delta \mathrm{OD}(t) * g(t)
$$

Nach der Normierung auf $\Delta \mathrm{OD}_{\max }$ wurde die Güte der Signalanpassung anhand der Residuen

$$
\operatorname{res}(t)=\Delta \mathrm{OD}_{\exp }(t)-S(t)
$$

beurteilt. Eine mehrmalige Iteration der Prozedur unter Variation einzelner Parameter lieferte schließlich für alle untersuchten Modellsysteme eine sehr genaue Anpassung an die experimentellen Daten. 


\subsection{Resultate der Auswertung}

\subsubsection{Messungen an quasi-isolierten Aromaten}

In diesem Abschnitt werden die Resultate der Signalanpassungen für die Messungen an den reinen aromatischen Modellsystemen präsentiert. Eine Gegenüberstellung der experimentellen und simulierten Signale samt der zugehörigen Residuen sind in den Abb. 6.1-6.5 zu finden. Die Parameter der Anpassungsfunktion F sind in den Tabellen 6.1-6.4 zusammengefaßst.

Die Modellierungsfunktion mit biexponentieller IVR-Dynamik und monoexponentiellem VET-Term führte in allen Fällen zu einer sehr guten Anpassung der experimentellen Absorptions-Zeit-Profile. Die Residuen der einzelnen Anpassungen am Benzol (Abb. 6.1-6.3) zeigen lediglich eine leichte systematische Abweichung nach unten im Bereich von 50-100 ps. Hier wäre unter Zuhilfenahme eines dritten IVR-Terms eine Verbesserung der Anpassung zwar möglich; allerdings würde dies die Anpassungsfunktion zu flexibel gestalten, so daß die Verläßlichkeit der absoluten Parameterwerte eingeschränkt würde.

Den simulierten Signalen des monodeuterierten Benzols liegt das Kalibrationspolynom des Benzols zugrunde (vgl. Abschn. 4.2). Die beiden transienten Absorptionsprofile bei 1677 bzw. $2148 \mathrm{~nm}$ Anregungswellenlänge konnten so ohne systematische Abweichungen mit Gl. (6.5) simuliert werden (Abb. 6.4).

Auch für die Messungen an Chlorbenzol, Toluol und $\alpha, \alpha, \alpha$-Trifluortoluol liefert die Anpassung mit biexponentiellem Anstieg und monoexponentiellem Rückgang der optischen Dichte hervorragende Ergebnisse, wie in Abb. 6.5 zu sehen ist. Nach den ersten fünf Pikosekunden kann von einem statistischen Verlauf der Residuen um die Nulllinie gesprochen werden. Schwierigkeiten bei der Reproduktion des frühen Absorptionsanstiegs sind auf die Überlagerung des Signals mit dem Kreuzkorrelationsartefakt zurückzuführen.

Eine ausführliche Diskussion der Auswirkung von Temperatur, Druck, Anregungsenergie und chemischer Konstitution auf die Zeitskalen der intramolekularen Energieumverteilung wird in Kap. 7 vorgenommen. 

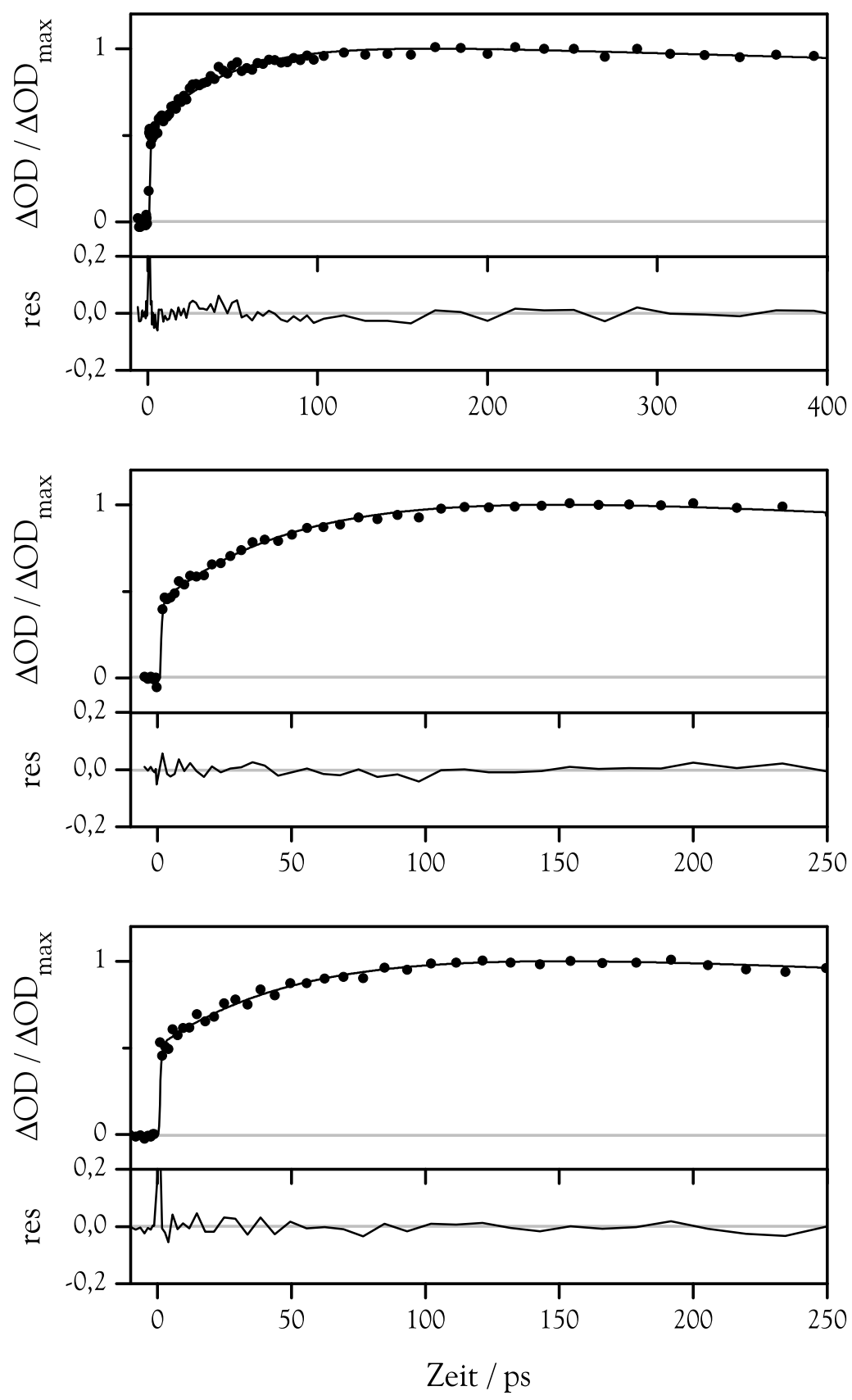

Abbildung 6.1: Anpassung der transienten Absorption von Benzol I (oben: $\lambda_{\text {Pump }}=1670 \mathrm{~nm}$; Mitte: $\lambda_{\text {Pump }}=2140 \mathrm{~nm}$; unten: $\mathrm{T}=80^{\circ} \mathrm{C}$ ) 

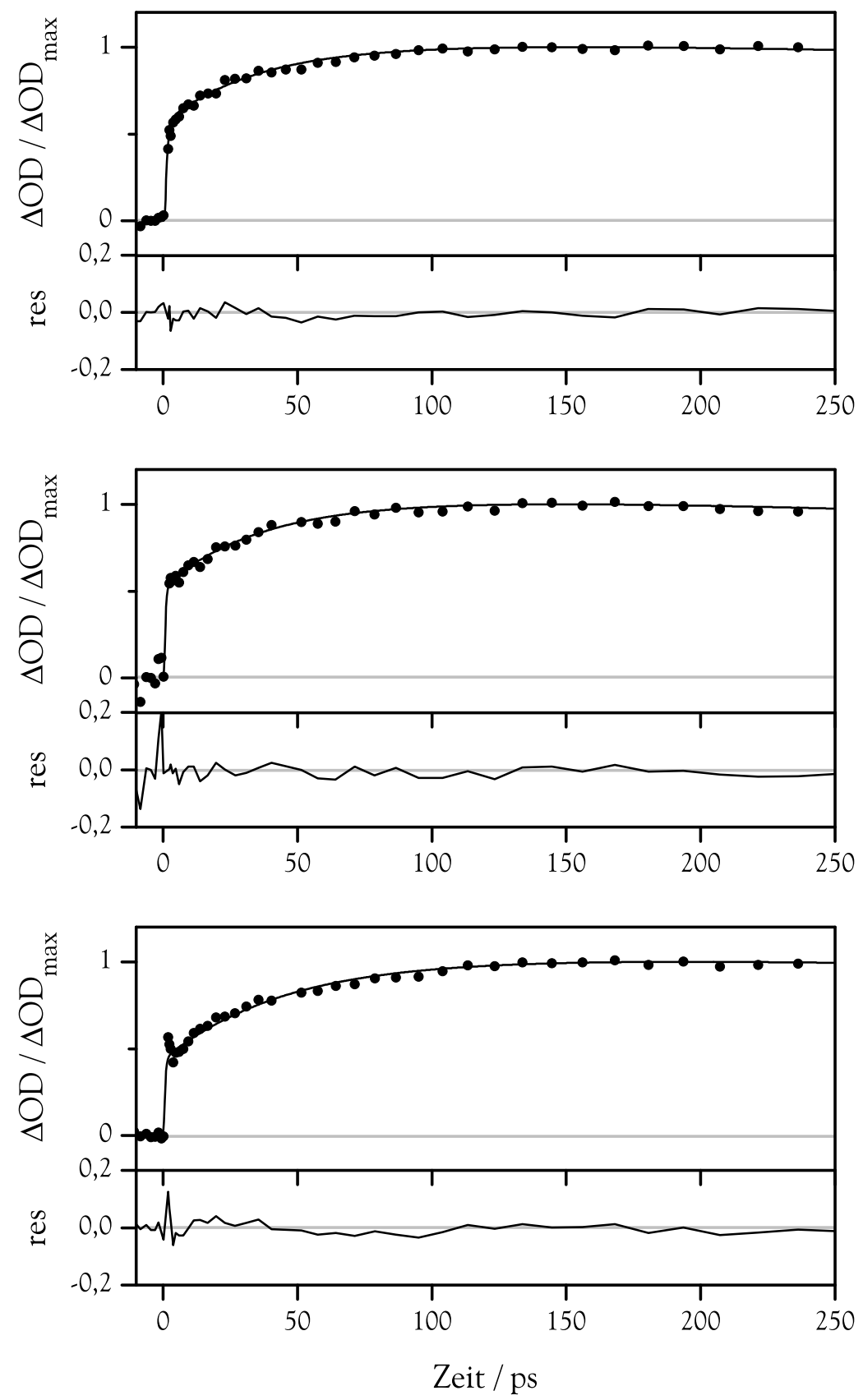

Abbildung 6.2: Anpassung der transienten Absorption von Benzol II (oben: $\mathrm{T}=120^{\circ} \mathrm{C}$; Mitte: $\mathrm{T}=140{ }^{\circ} \mathrm{C}$; unten: $\mathrm{T}=155^{\circ} \mathrm{C}$ ) 

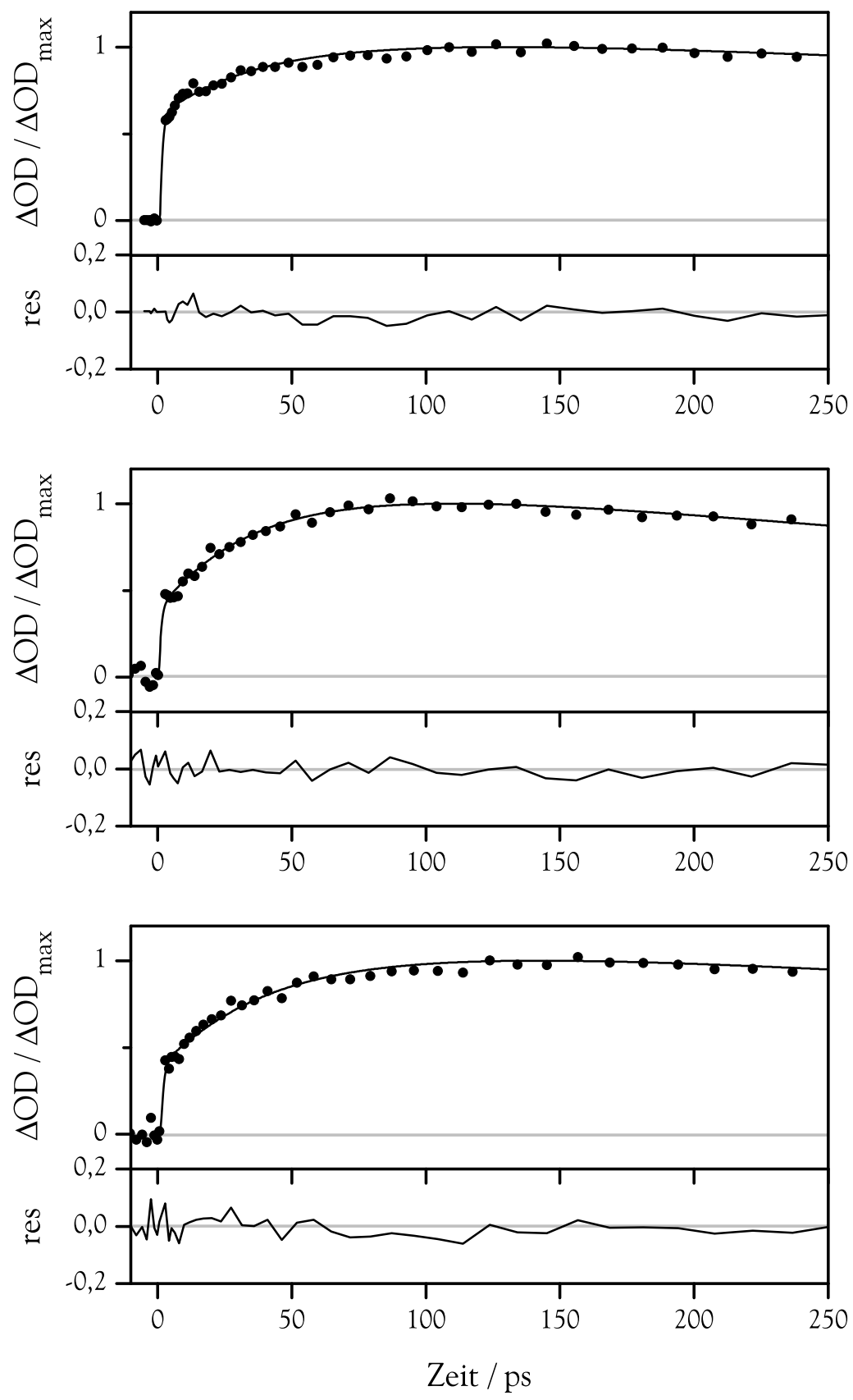

Abbildung 6.3: Anpassung der transienten Absorption von Benzol III (oben: $\mathrm{p}_{\text {Benzol }}=1.0$ bar; Mitte: $\mathrm{p}_{\text {Benzol }}=2.3$ bar; unten: $\mathrm{p}_{\text {Benzol }}=3.3$ bar $)$ 

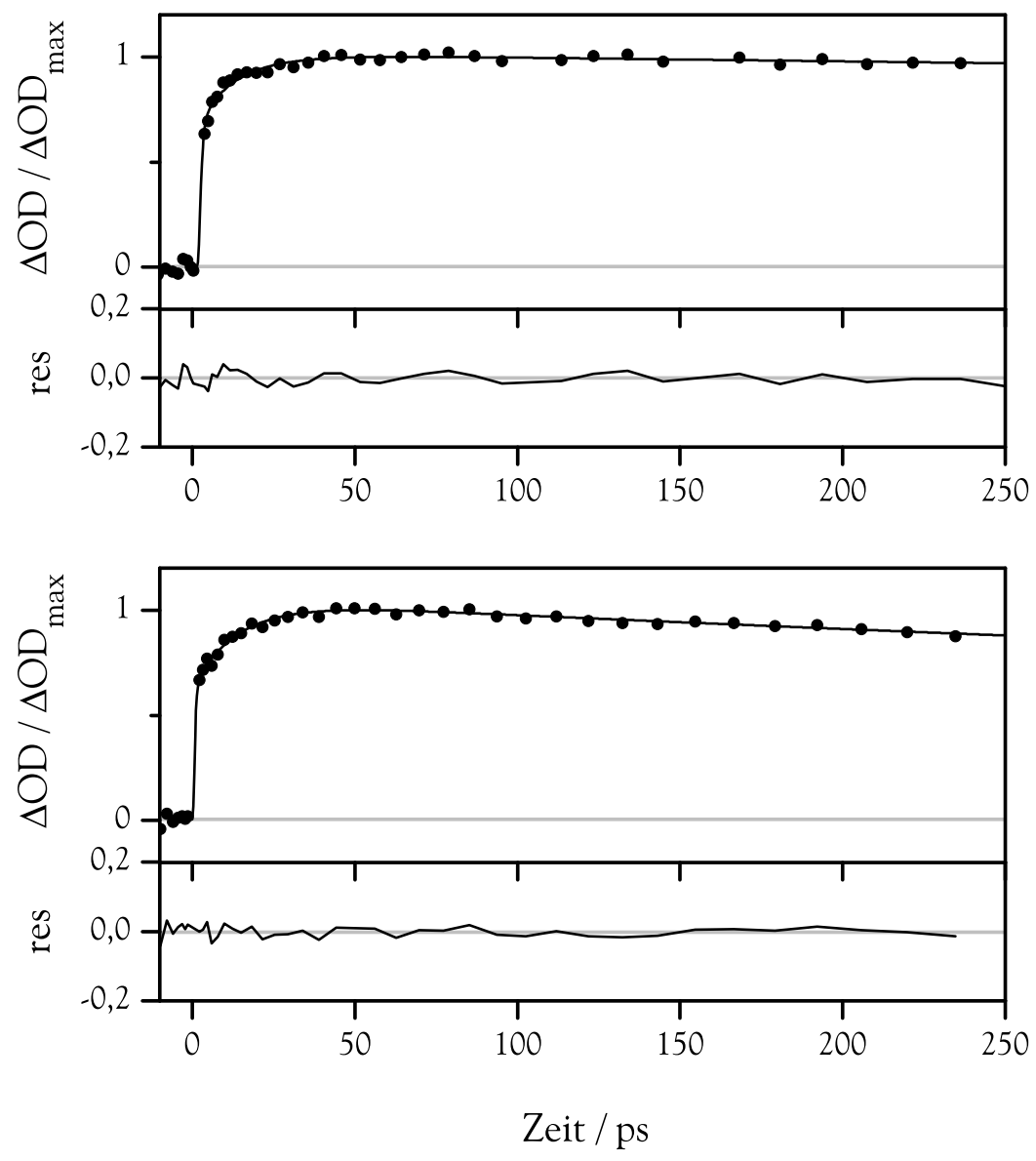

Abbildung 6.4: Anpassung der transienten Absorption von Benzol- $\mathrm{d}_{1}$ (oben: $\lambda_{\text {Pump }}=1677 \mathrm{~nm}$; unten: $\lambda_{\text {Pump }}=2148 \mathrm{~nm}$ ) 

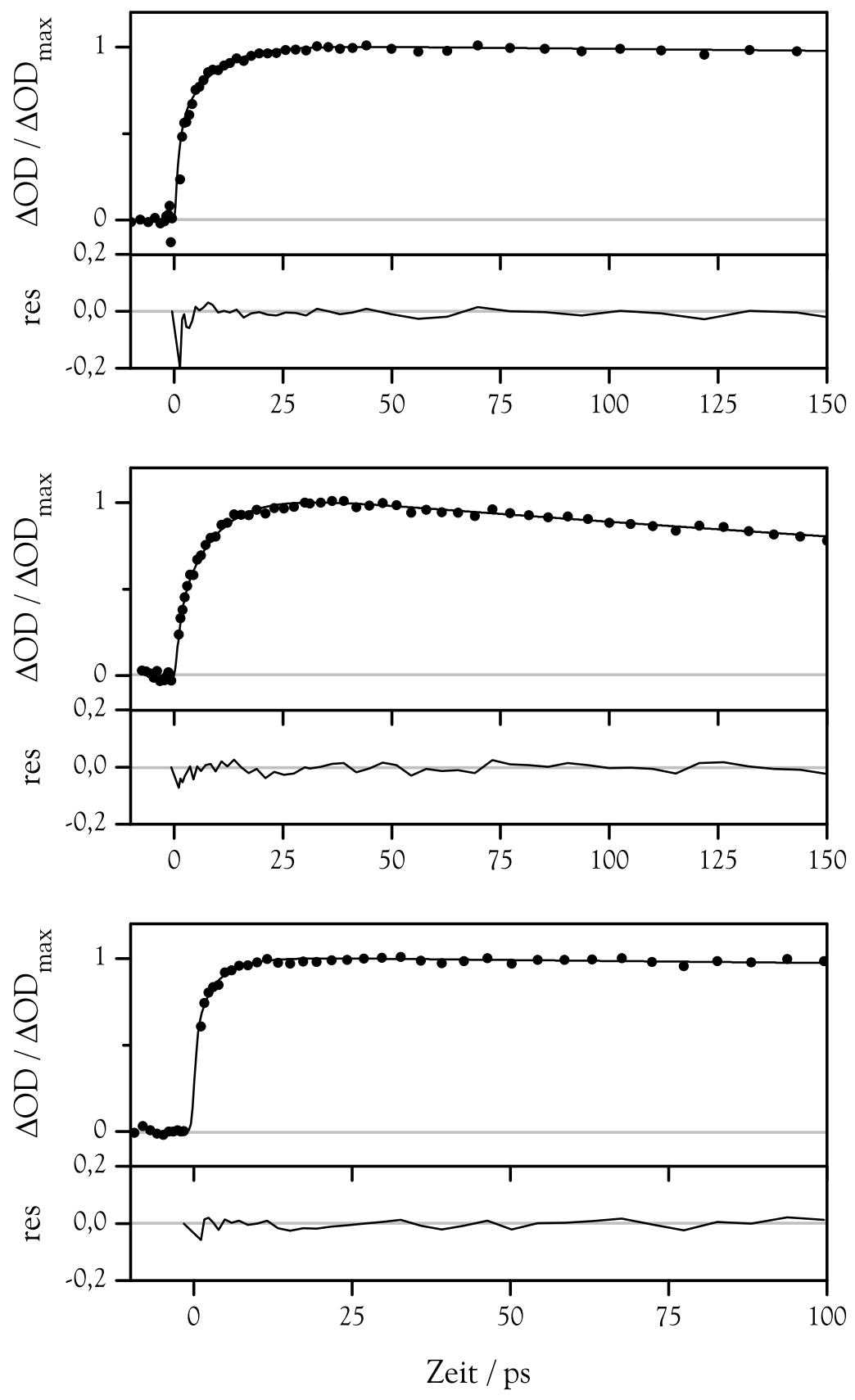

Abbildung 6.5: Anpassung der transienten Absorption von Chlorbenzol (oben), Toluol (Mitte) und $\alpha, \alpha, \alpha$-Trifluortoluol (unten) 
Kapitel 6 Auswertung

\begin{tabular}{|lcccc|}
\hline $\boldsymbol{\lambda}_{\text {Pump }}$ & $\boldsymbol{\tau}_{\mathbf{I V R}_{\mathbf{1}}} / \mathbf{p s}$ & $\boldsymbol{\tau}_{\mathbf{I V R}_{\mathbf{2}}} / \mathbf{p s}$ & $\mathbf{A}_{\text {inst }}$ & $\boldsymbol{\tau}_{\text {VET }} / \mathbf{p s}$ \\
\hline \hline $1670 \mathrm{~nm}$ & 0.5 & $48 \pm 5$ & $0.45 \pm 0.05$ & $4000 \pm 500$ \\
$2140 \mathrm{~nm}$ & 0.5 & $56 \pm 5$ & $0.41 \pm 0.05$ & $1100 \pm 100$ \\
\hline
\end{tabular}

Tabelle 6.1: Zeitkonstanten für die Anpassungen der Messungen an Benzol bei verschiedenen Anregungswellenlängen

\begin{tabular}{|ccccc|}
\hline Temperatur & $\boldsymbol{\tau}_{\text {IVR }_{\mathbf{1}}} / \mathbf{p s}$ & $\boldsymbol{\tau}_{\mathbf{I V R}_{\mathbf{2}}} / \mathbf{p s}$ & $\mathbf{A}_{\text {inst }}$ & $\boldsymbol{\tau}_{\text {VET }} / \mathbf{p s}$ \\
\hline \hline $80^{\circ} \mathrm{C}$ & 0.5 & $52 \pm 6$ & $0.43 \pm 0.05$ & $1100 \pm 100$ \\
$100^{\circ} \mathrm{C}$ & 0.5 & $48 \pm 5$ & $0.45 \pm 0.05$ & $4000 \pm 500$ \\
$120^{\circ} \mathrm{C}$ & 0.5 & $47 \pm 5$ & $0.47 \pm 0.05$ & $2200 \pm 200$ \\
$140^{\circ} \mathrm{C}$ & 0.5 & $50 \pm 5$ & $0.43 \pm 0.05$ & $1500 \pm 200$ \\
$155^{\circ} \mathrm{C}$ & 0.5 & $54 \pm 6$ & $0.37 \pm 0.05$ & $2000 \pm 200$ \\
\hline
\end{tabular}

Tabelle 6.2: Zeitkonstanten für die Anpassungen der Messungen an Benzol bei verschiedenen Meßstemperaturen

\begin{tabular}{|ccccc|}
\hline $\mathbf{p}_{\text {Benzol }}$ & $\boldsymbol{\tau}_{\mathbf{I V R}_{\mathbf{1}}} / \mathbf{p s}$ & $\boldsymbol{\tau}_{\mathbf{I V R}_{\mathbf{2}}} / \mathbf{p s}$ & $\mathbf{A}_{\text {inst }}$ & $\boldsymbol{\tau}_{\mathbf{V E T}} / \mathbf{p s}$ \\
\hline \hline $0.5 \mathrm{bar}$ & 0.5 & $48 \pm 5$ & $0.45 \pm 0.05$ & $4000 \pm 500$ \\
$1.0 \mathrm{bar}$ & 0.5 & $50 \pm 5$ & $0.47 \pm 0.05$ & $1100 \pm 100$ \\
$2.3 \mathrm{bar}$ & 0.5 & $45 \pm 5$ & $0.27 \pm 0.05$ & $580 \pm 60$ \\
$3.3 \mathrm{bar}$ & 0.5 & $53 \pm 5$ & $0.28 \pm 0.05$ & $900 \pm 80$ \\
\hline
\end{tabular}

Tabelle 6.3: Zeitkonstanten für die Anpassungen der Messungen an Benzol bei verschiedenen Benzoldrücken 
6.2 Resultate der Auswertung

\begin{tabular}{|lcccc|}
\hline Modellsystem & $\boldsymbol{\tau}_{\mathbf{I V R}_{\mathbf{1}}} / \mathbf{p s}$ & $\boldsymbol{\tau}_{\mathbf{I V R}_{\mathbf{2}}} / \mathbf{p s}$ & $\mathbf{A}_{\text {inst }}$ & $\boldsymbol{\tau}_{\text {VET }} / \mathbf{p s}$ \\
\hline \hline \multicolumn{4}{c}{$\lambda_{\text {Pump }}$} & $\approx 1670 \mathrm{~nm}$ \\
Benzol-d & & & \\
Chlorbenzol & 0.5 & $14 \pm 2$ & $0.61 \pm 0.05$ & $3500 \pm 500$ \\
Toluol & 0.5 & $6.5 \pm 0.8$ & $0.65 \pm 0.05$ & $6000 \pm 800$ \\
$\alpha, \alpha, \alpha$-Trifluortoluol & 0.8 & $8.2 \pm 0.8$ & $0.30 \pm 0.05$ & $600 \pm 70$ \\
& 0.8 & $3.8 \pm 0.5$ & $0.65 \pm 0.05$ & $2800 \pm 400$ \\
Benzol-d $_{1}$ & \multicolumn{2}{c}{$\lambda_{\text {Pump }}=2148 \mathrm{~nm}$} & & \\
& 0.5 & $16 \pm 2$ & $0.60 \pm 0.05$ & $1200 \pm 100$ \\
\hline
\end{tabular}

Tabelle 6.4: Zeitkonstanten für die Anpassungen der Messungen an Benzol- $\mathrm{d}_{1}$, Chlorbenzol, Toluol und $\alpha, \alpha, \alpha$-Trifluortoluol

\subsubsection{Messungen an Benzol-Inertgas-Mischungen}

Dieser Abschnitt widmet sich den Ergebnissen der $\Delta$ OD-Simulationen von Benzol in den verschiedenen gasförmigen und überkritischen Umgebungen. In den Abb. 6.6-6.8 sind jeweils die simulierten den experimentellen Signalen gegenübergestellt. Zur besseren Bewertung der Anpassungsgüte sind auch die jeweiligen Residuen gezeigt. Die Parameter, welche die Signale jeweils optimal reproduzieren, sind in den Tabellen 6.5-6.7 am Ende dieses Abschnitts aufgelistet.

Im Vergleich zu den reinen Gasphasenmessungen zeichnen sich die transienten Absorptionen der Benzol-Inertgas-Messungen oftmals durch ein kleineres Signalzu-Rausch-Verhältnis aus. Dennoch kann in allen Fällen die Anwendbarkeit des oben formulierten kinetischen Modells auf die zugrundeliegende Schwingungsdynamik eindeutig beurteilt werden.

Die Reproduktion des Signalanstiegs und -maximums mit Hilfe des biexponentiellen IVR-Ansatzes gelingt in allen Fällen sehr gut. Abweichungen im Bereich des Signalursprungs können durch die überlagerte Kreuzkorrelationsabsorption erklärt werden und beeinflußen die ermittelten IVR-Zeitskalen nur marginal.

Auch die auf intermolekularen Energietransfer zurückzuführende Absorptionabnahme zu langen Verzögerungszeiten wird bis auf zwei Fälle in zufriedenstellender Weise wiedergegeben. Die Ausnahmen bilden die Messungen in überkritischem $\mathrm{CO}_{2}$ bei 100 bar (Abb. 6.7, unten) und in überkritischem $\mathrm{C}_{5} \mathrm{~F}_{12}$ bei 19 bar (Abb. 6.8, unten). Hier treten bei ca. 100 ps systematische Abweichungen der Residuen nach oben auf, was auf einen multiexponentiellen Charakter der Ab- 
sorptionsabnahme hinweist. Dieser wäre wahrscheinlich durch Inkorporation eines zweiten VET-Terms in Gl. (6.5) zu erfassen. Ein solcher Ansatz hat sich bereits für die Simulation der transienten Absorptionen von Aromaten in der kondensierten Phase bewährt [93-95]. Allerdings würde eine derartige Erweiterung zum einen die Anpassungsfunktion zu flexibel werden lassen und zum anderen die Vergleichbarkeit der Zeitkonstanten mindern. Es wurde daher auf eine exakte Simulation des VET-Anteils dieser beiden Signale verzichtet. Die dokumentierten Zeitkonstanten $\tau_{\text {VET }}$ können dennoch als effektive Gesamtrelaxationszeiten in die Diskussion einfließen. 

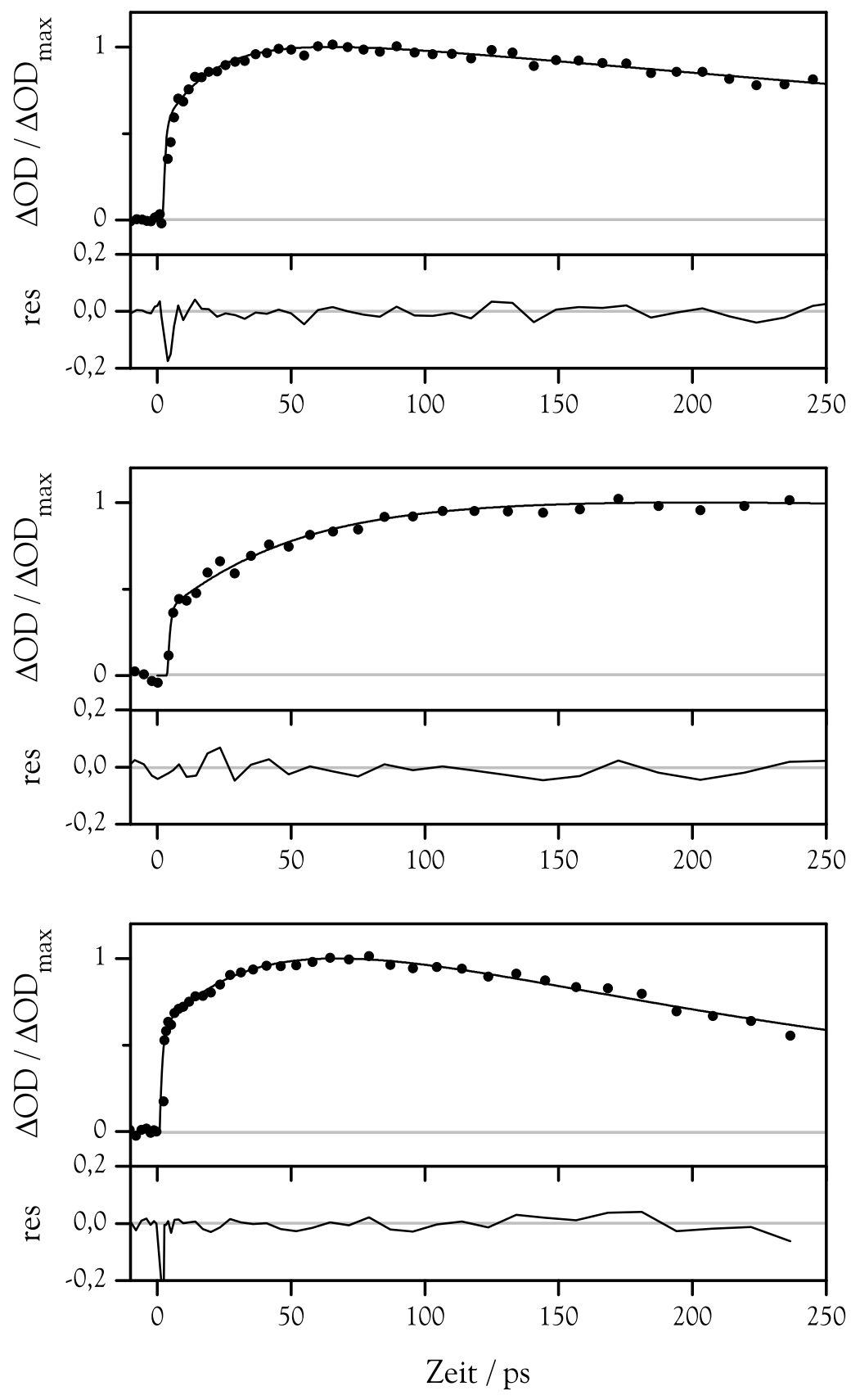

Abbildung 6.6: Anpassung der transienten Absorption von Benzol in Helium (oben), Argon (Mitte) und 1,1,2-Trichlortrifluorethan (unten) 

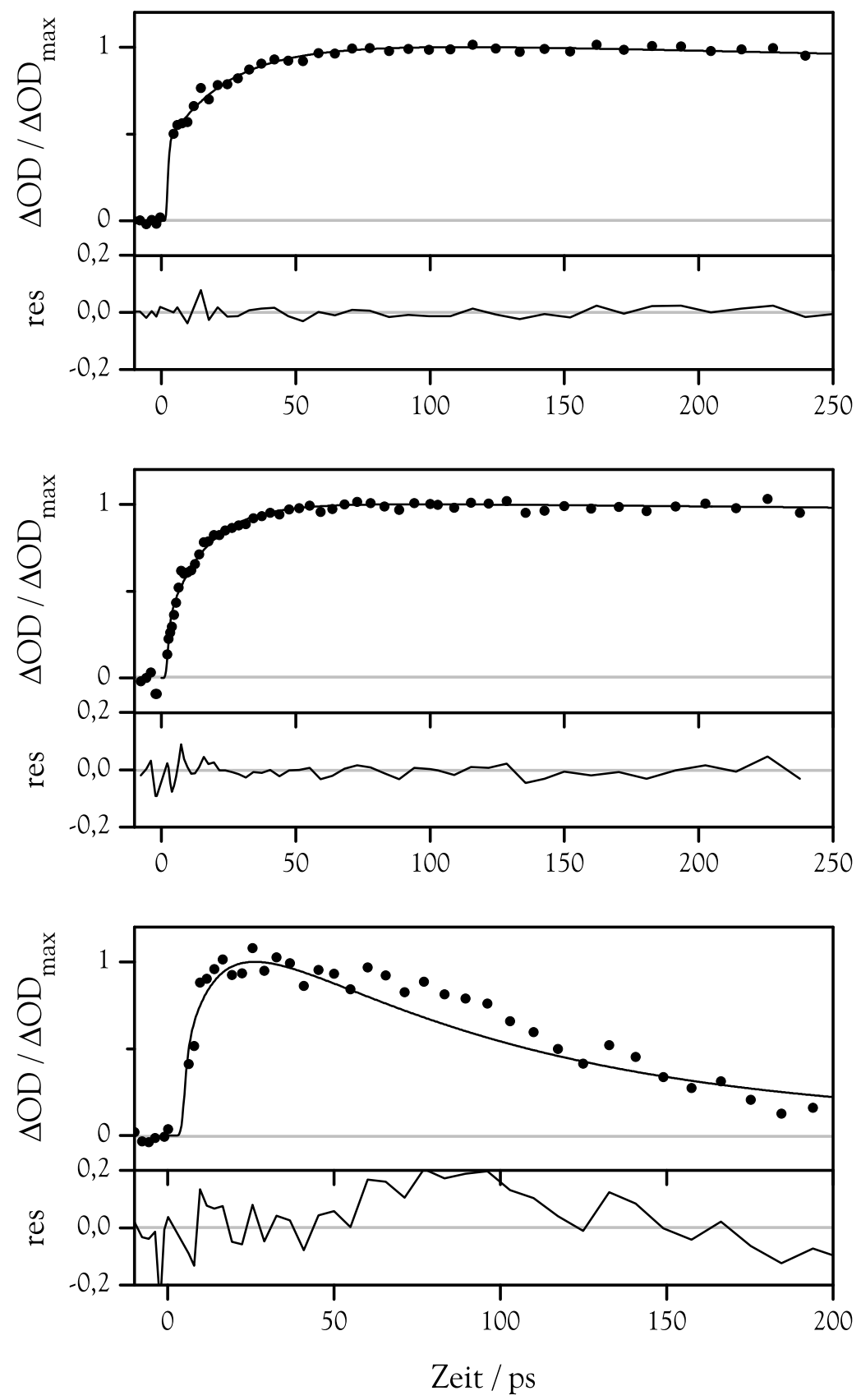

Abbildung 6.7: Anpassung der transienten Absorption von Benzol in $\mathrm{CO}_{2}$ (oben: $\mathrm{p}_{\mathrm{CO}_{2}}=10$ bar; Mitte: $\mathrm{p}_{\mathrm{CO}_{2}}=18$ bar; unten: $\mathrm{p}_{\mathrm{CO}_{2}}=100$ bar $)$ 

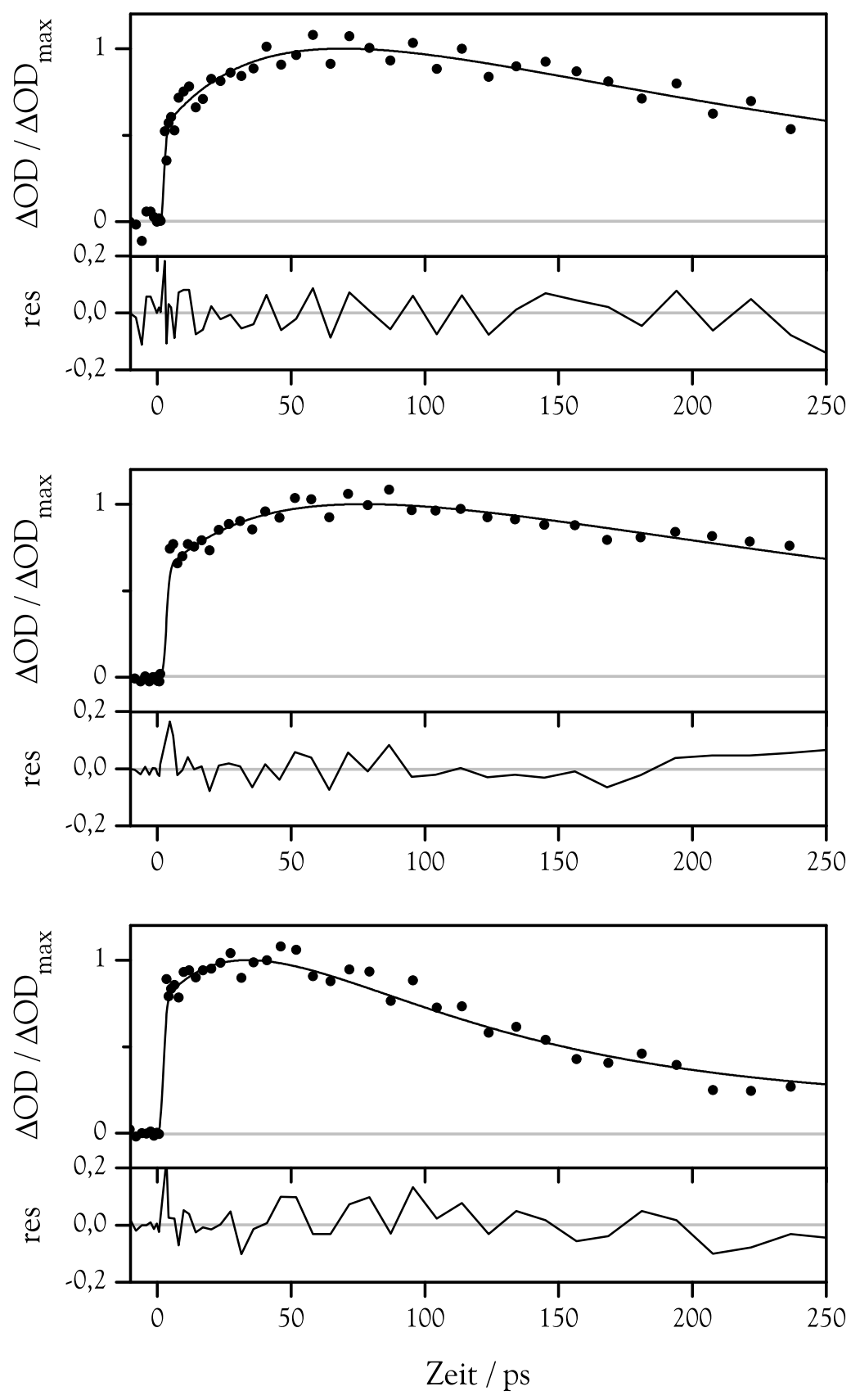

Abbildung 6.8: Anpassung der transienten Absorption von Benzol in $\mathrm{C}_{5} \mathrm{~F}_{12}$ (oben: $\mathrm{p}_{\mathrm{C}_{5} \mathrm{~F}_{12}}=6$ bar; Mitte: $\mathrm{p}_{\mathrm{C}_{5} \mathrm{~F}_{12}}=15$ bar; unten: $\mathrm{p}_{\mathrm{C}_{5} \mathrm{~F}_{12}}=19$ bar) 


\begin{tabular}{|lcccc|}
\hline Inertgas & $\boldsymbol{\tau}_{\mathbf{I V R}_{\mathbf{1}}} / \mathbf{p s}$ & $\boldsymbol{\tau}_{\mathbf{I V R}_{\mathbf{2}}} / \mathbf{p s}$ & $\mathbf{A}_{\text {inst }}$ & $\boldsymbol{\tau}_{\mathbf{V E T}} / \mathbf{p s}$ \\
\hline \hline Helium & 0.5 & $23 \pm 3$ & $0.45 \pm 0.05$ & $580 \pm 80$ \\
Argon & 0.5 & $62 \pm 5$ & $0.32 \pm 0.05$ & $2000 \pm 200$ \\
$\begin{array}{l}\text { 1,1,2-Trichlor- } \\
\text { trifluorethan }\end{array}$ & 0.5 & $42 \pm 5$ & $0.34 \pm 0.05$ & $230 \pm 50$ \\
\hline
\end{tabular}

Tabelle 6.5: Zeitkonstanten für die Anpassungen der Messungen an Benzol in verschiedenen Badgasen

\begin{tabular}{|ccccc|}
\hline $\boldsymbol{\rho}_{\text {red,CO}}$ & $\boldsymbol{\tau}_{\mathbf{I V R}_{\mathbf{1}}} / \mathbf{p s}$ & $\boldsymbol{\tau}_{\mathbf{I V R}_{\mathbf{2}}} / \mathbf{p s}$ & $\mathbf{A}_{\text {inst }}$ & $\boldsymbol{\tau}_{\mathbf{V E T}} / \mathbf{p s}$ \\
\hline \hline 0.03 & 0.5 & $29 \pm 5$ & $0.39 \pm 0.05$ & $2000 \pm 500$ \\
0.06 & 0.5 & $18 \pm 3$ & $0.29 \pm 0.05$ & $4500 \pm 500$ \\
0.4 & 0.5 & $10 \pm 3$ & $0.30 \pm 0.05$ & $100 \pm 20$ \\
\hline
\end{tabular}

Tabelle 6.6: Zeitkonstanten für die Anpassungen der Messungen an Benzol in überkritischem $\mathrm{CO}_{2}$

\begin{tabular}{|ccccc|}
\hline $\boldsymbol{\rho}_{\text {red, } \mathbf{C}_{\mathbf{5}} \mathbf{F}_{\mathbf{1 2}}}$ & $\boldsymbol{\tau}_{\mathbf{I V R}_{\mathbf{1}}} / \mathbf{p s}$ & $\boldsymbol{\tau}_{\mathbf{I V R}_{\mathbf{2}}} / \mathbf{p s}$ & $\mathbf{A}_{\text {inst }}$ & $\boldsymbol{\tau}_{\mathbf{V E T}} / \mathbf{p s}$ \\
\hline \hline 0.09 & 0.5 & $40 \pm 5$ & $0.34 \pm 0.05$ & $250 \pm 50$ \\
0.28 & 0.5 & $45 \pm 5$ & $0.41 \pm 0.05$ & $310 \pm 50$ \\
0.42 & 0.5 & $30 \pm 3$ & $0.37 \pm 0.05$ & $100 \pm 20$ \\
\hline
\end{tabular}

Tabelle 6.7: Zeitkonstanten für die Anpassungen der Messungen an Benzol in überkritischem $\mathrm{C}_{5} \mathrm{~F}_{12}$ 


\section{Kapitel 7}

\section{Diskussion}

Die IR-Pump-UV-Probe-Spektroskopie stellt ein vielseitig einsetzbares Werkzeug dar, um die Relaxation lokal schwingungsangeregter Moleküle zeitaufgelöst zu studieren. Dabei lassen die Ergebnisse einzelner Experimente an quasi-isolierten Molekülen in der Gasphase bereits erste Rückschlüsse auf die zugrundeliegenden Mechanismen der Energieumverteilung zu. Doch erst in Reihenuntersuchungen an chemisch ähnlichen Modellsystemen wird das Potential der transienten Absorptionstechnik voll ausgenutzt. Durch den Vergleich mit der Dynamik eines „Referenzsystems" können intramolekulare Relaxationskanäle identifiziert und ihre Beeinflussung durch chemische Faktoren beleuchtet werden.

In der vorliegenden Arbeit wurde eine Vielzahl chemischer Faktoren hinsichtlich ihres Einflusses auf die intramolekulare Schwingungsenergieumverteilung in aromatischen Molekülen untersucht. Die Diskussion der einzelnen Effekte gliedert sich in zwei Teile. Der erste Abschnitt widmet sich der Auswirkung der chemischen Konstitution eines angeregten Moleküls auf seine IVR-Dynamik. Anschließend wird die Rolle der molekularen Umgebung und ihrer chemischen Natur für die intramolekulare Relaxation erörtert.

\subsection{IVR in quasi-isolierten Molekülen}

\subsubsection{Benzol}

In der vorliegenden Arbeit stellt Benzol $\mathrm{C}_{6} \mathrm{H}_{6}$ das Referenzsystem dar. Die Ergebnisse der Pump-Probe-Messungen an quasi-isolierten Benzolmolekülen in der Gasphase belegen eindeutig, daß die intramolekulare Umverteilung der Schwingungsenergie nach einer $2 v_{\mathrm{CH} \text {-streck-Anregung }}\left(\lambda_{\mathrm{Pump}}=1670 \mathrm{~nm}\right)$ in mindestens 
zwei Schritten abläuft. Die erste IVR-Komponente liegt im Subpikosekundenbereich $\left(\tau_{\mathrm{IVR}_{2}}<0.5 \mathrm{ps}\right)$, während die zweite mit einer Zeitkonstante von $\tau_{\mathrm{IVR}_{2}}=$ $48 \pm 5$ ps um zwei Größenordnungen langsamer fortschreitet. Die Existenz weiterer IVR-Zeitskalen ist anhand der experimentellen Daten nicht auszuschließen. Jedoch führt der biexponentielle IVR-Ansatz des auf S. 139 eingeführten kinetischen Modells bereits zu einer sehr zufriedenstellenden Reproduktion der transienten Absorptionssignale, so daß sich die Diskussion des intramolekularen Energieflusses für Benzol im folgenden auf die beiden oben genannten Zeitskalen konzentriert.

Die bloße Existenz mehrerer IVR-Zeitskalen beweist bereits den nicht-statistischen Charakter des IVR-Prozesses im Benzol. Im Falle einer gleichberechtigten Kopplung aller isoenergetischen Hintergrundzustände an den hellen Zustand, wie sie Fermis Goldener Regel entspräche, wäre lediglich eine monoexponentielle IVR-Dynamik zu erwarten. In dem Referenzsystem Benzol ist der Energiefluß hingegen offensichtlich sequentieller Natur. Eine Erklärung, warum sich dieser hierarchische Charakter in den zeitaufgelösten Pump-Probe-Experimenten wiederspiegelt, bietet das in Abschn. 2.1.2 vorgestellte IVR-Stufenmodell. Die Absorption des Probepulses hängt von der Besetzung weniger FC-aktiver Normalmoden des Moleküls ab. Sind nun diese Moden an $\mathrm{zu}$ verschiedenen Umverteilungsstufen gehörenden Zuständen beteiligt, ändert sich ihre Anregung im Zuge der intramolekularen Relaxationskaskade mit der Geschwindigkeit, die dem Energiefluß durch die IVR-Stufen entspricht. Die zweistufige Zunahme der transienten Absorption, welche bei Benzol beobachtet wurde, läßt somit vermuten, daß mindestens zwei unterschiedliche Untergruppen von Zuständen mit dem Probepuls abgefragt werden. Allerdings kann anhand der zeitaufgelösten Messungen nicht beurteilt werden, ob es sich um verschiedene FC-aktive Moden in verschiedenen IVR-Stufen handelt.

Ein Vergleich mit zustandsaufgelösten Gasphasenspektren des Benzols im Bereich des ersten Obertons der CH-Streckschwingungen zeigt die Konkurrenzfähigkeit der zeitaufgelösten mit der frequenzaufgelösten Spektroskopie auf. In IR-Sättigungsexperimenten an Benzol in Überschallexpansionen bestimmten Page et al. die Bandenbreite des $(v=2)$-Übergangs $\mathrm{zu} \tilde{\Gamma} \approx 30 \mathrm{~cm}^{-1}$, woraus sich über

$$
\tau=(2 \pi c \tilde{\Gamma})^{-1}
$$

eine Lebensdauer des ersten CH-Streckobertons von ca. 200 fs ergab [64]. Quack und Mitarbeiter wandten (IR $+\mathrm{UV})$-Doppelresonanzspektroskopie an und identi- 
7.1 IVR in quasi-isolierten Molekülen

fizierten mittels Linienbreitenanalysen drei Zeitskalen der intramolekularen Redistribution in Benzol, wovon zwei im Subpikosekundenbereich liegen $(\tau \approx 100$ bzw. $350 \mathrm{fs}$ ), was mit dem Ergebnis von Page gut übereinstimmt. Die dritte Zeitkonstante wird von ihnen als Untergrenze eines langsamen IVR-Schrittes mit $\tau>1.3 \mathrm{ps}$ angegeben [100]. Spektrale Signaturen einer langsamen IVRKomponente für $\mathrm{S}_{0}$-Benzol fanden sich ebenfalls in den rotationsaufgelösten Fluoreszenz-Spektren von Nicholson und Lawrance, die der irreversiblen Umverteilung eine untere Grenze von 21 ps zuwiesen [101]. Schmalere Linienprofile der Ringmoden lassen sogar deutlich längere IVR-Lebensdauern vermuten. Auch Scoles und Mitarbeiter beobachteten in IR-IR-Doppelresonanzspektren einen zweiten, langsamen IVR-Schritt, der je nach Rotationsanregung des hellen Zustands 10-20 ps dauerte [58]. Die Übereinstimmung der in der Frequenzdomäne ermittelten IVR-Zeitskalen mit den im Rahmen dieser Arbeit zeitaufgelöst bestimmten ist bemerkenswert.

Die scheinbare Diskrepanz in der langsamen Zeitskala (ca. Faktor 2) ist auf die unterschiedlichen Beobachtungsfenster der experimentellen Methoden zurückzuführen. Zum einen handelt es sich bei der transient gemessenen Zeitkonstante $\tau_{\mathrm{IVR}_{2}}$ möglicherweise um einen Mittelwert mehrerer langsamer IVR-Schritte. Zum anderen sind in frequenzaufgelösten Untersuchungen nur helle - und damit allenfalls intermediäre - Zustände zugänglich, so daß die aus Linienbreiten bestimmten Zeitskalen nur untere Grenzen darstellen können.

Der große Unterschied in den Zeitskalen der IVR-Komponenten legt nahe, daß der helle Zustand besser an die Zustände der ersten IVR-Stufe koppelt als diese an die restlichen Hintergrundzustände. In Anbetracht der bekannten Fermiresonanz zwischen den in-plane CH-Streck- und CH-Biegeschwingungen des Benzols (s. Abschn. 4.1) ist es verlockend, dem ersten Satz von Zuständen die Schwingungen innerhalb der Molekülebene zuzuordnen. Schließlich konnten theoretische Modelle, deren Hamiltonian lediglich ein planares Benzolmolekül zuließ, die Charakteristiken der Spektren aus [64] gut reproduzieren [103, 104]. Auch Wyatt et al. zeigten in quantendynamischen Simulationen zur $2 v_{\mathrm{CH}-\text { streck }}$-Relaxation, daß der Hauptteil der Anregungsenergie (35\%) innerhalb der ersten 200 fs von den $\mathrm{CH}-$ Biegeschwingungen parallel zur Molekülebene aufgenommen wird. Allerdings stellten sie bei Berücksichtung aller 30 Benzolschwingungen darüber hinaus eine beträchtliche Beteiligung (20\%) von out-of-plane-Schwingungen an der frühen Umverteilung fest $[102,105]$. Somit stellt die Interpretation der ersten IVR-Stufe 
als in-plane-Fermi-Resonanz lediglich eine (physikalisch durchaus sinnvolle) Näherung dar.

Wyatts Berechnungen ergaben weiterhin, daß IVR in Benzol nach 9.6 ps nicht abgeschlossen ist, sondern unter zunehmender Population der niederfrequenten Ringmoden auf einer Pikosekundenzeitskala fortschreitet. Folglich können spätere IVR-Stufen durch eine verstärkte Beteiligung dieser Schwingungen charakterisiert werden.

Ein Blick auf die Simulationsergebnisse für die FC-aktiven Moden $\nu_{27}$ $\left(608 \mathrm{~cm}^{-1}\right)$ und $\nu_{29}\left(398 \mathrm{~cm}^{-1}\right)$ zeigt, daß sie in sehr unterschiedlicher Weise an der Umverteilung der Anregungsenergie teilnehmen (Abb. 7.1). Der Energiegehalt von $\nu_{29}$ nimmt innerhalb der ersten Pikosekunde stark zu und verbleibt für die restliche Simulationzeit auf einem konstanten Niveau. Im Gegensatz hierzu ist für $\nu_{27}$ ein steter Zuwachs an Energie über die gesamte Simulationsdauer zu vermerken. Unter der Annahme, daß beide Moden zum Pump-Probe-Signal des Benzols beitragen, kann von einer sehr guten Übereinstimmung der experimentell beobachteten Trends mit den theoretischen Berechnungen gesprochen werden.

Die Amplitude $\mathrm{A}_{\text {inst }}$ ist direkt mit der relativen Beteiligung der beiden IVR-Komponenten am Gesamtprozess korreliert. Eine solche Größe ist in der Frequenzdomäne nicht zugänglich. Im Falle des Benzols haben die beiden IVR-

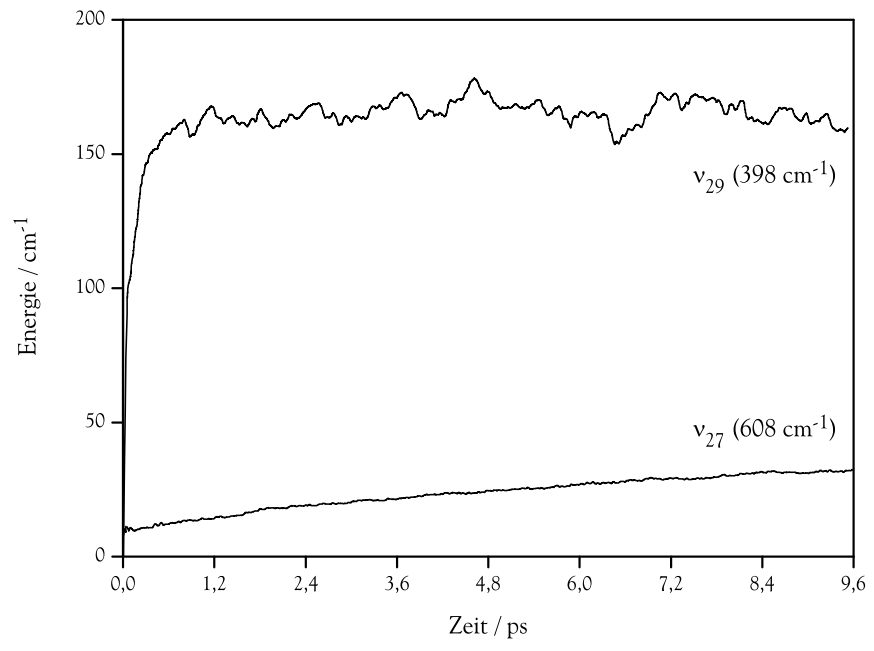

Abbildung 7.1: Quantendynamische Simulation von Wyatt et al., übernommen aus [102] 
7.1 IVR in quasi-isolierten Molekülen

Schritte ungefähr gleich großen Anteil an der Umverteilung der Anregungsenergie auf die FC-aktiven Moden des Moleküls.

\section{Energieabhängigkeit}

In den Pump-Probe-Experimenten an Benzol wurden mit IR-Pulsen unterschiedlicher Energie selektiv helle Zustände präpariert, die entweder als reine $\mathrm{CH}$-Streckbewegung oder gemischte $\mathrm{CH}$ - und CC-Streckschwingung beschrieben werden können. Auf diese Weise kann untersucht werden, ob und wie die Natur des ZOBS die Stärke der intramolekulare Kopplungen beeinflußt. Es sei an dieser Stelle nochmals darauf hingewiesen, daß es sich auch bei dem ZOBS mit $6000 \mathrm{~cm}^{-1}$ Überschußenergie um einen Kombinationston verschiedener CH-Streckschwingungen handelt, da die direkte Anregung von Obertönen mit gerader Quantenzahl in der Punktgruppe des Benzols verboten ist (vgl. Abschn. 4.1).

Bei Benzol wurde für den schnellen IVR-Schritt innerhalb der limitierenden Zeitauflösung keine Änderung nach Variation der Anregungsenergie beobachtet. Die zweite, langsame IVR-Komponente weist dagegen eine Verlangsamung von mehr als $10 \%$ bei Verwendung des niederenergetischeren Pumppulses auf. Gleichzeitig geht der Anteil des schnellen Schrittes an der gesamten intramolekularen Relaxation von 0.45 auf 0.41 zurück.

Die leicht energieabhängige Dynamik läßt darauf schließen, daß der helle Zustand mit $2 v_{\mathrm{CH}}$-Charakter tendentiell besser an die Hintergrundzustände koppelt als die $v_{\mathrm{CH}} v_{\mathrm{CC}}-$ Kombination. Da beide ZOBS im QNS als surface states gelten (Abschn. 2.1.3), ist die Dimension des für die Kopplung verfügbaren Zustandsraums in beiden Fällen gleich. Die Anregungsenergie wird jeweils instantan über zwei Schwingungsfreiheitsgrade delokalisiert. Somit bietet die bloße Lage der Zustände im QNS für Benzol keinen zufriedenstellenden Erklärungsansatz für die schnellere Relaxation der reinen CH-Streckschwingung.

Allerdings bestimmt neben der Delokalisierung der Überschußenergie auch die Anharmonizität der angeregten Mode die Güte der intramolekularen Kopplungen. Die relative Rotverschiebung der entsprechenden Banden im IRAbsorptionsspektrum zeigt, daß die Anharmonizität der $2 v_{\mathrm{CH}^{-}}$Schwingungen generell stärker ausgeprägt ist als die der $v_{\mathrm{CH}} v_{\mathrm{CC}}$-Moden. Dies sollte die Kopplung der reinen CH-Streckanregung an die dunklen Hintergrundzustände begünstigen. 
Darüber hinaus liegt $\mathrm{zu}$ dem höherenergetischen $2 v_{\mathrm{CH}^{-}}$-Kombinationston eine zehnmal größere Gesamtanzahl von Hintergrundzuständen vor, wie der energieabhängigen Schwingungszustandsdichte des Moleküls auf S. 91 entnommen werden kann. Damit steigt die Wahrscheinlichkeit von Kopplungen über Resonanzen niedriger Ordnung, wodurch die intramolekulare Umverteilung ebenfalls beschleunigt wird.

Somit ist die schnellere IVR-Dynamik bei einer Anregungswellenlänge von $1670 \mathrm{~nm}$ sowohl auf die stärkere Ausprägung als auch auf die höhere Anzahl intramolekularer Kopplungen der reinen CH-Streckschwingung zurückzuführen.

Ein analoger Trend wurde auch im Fall des monodeuterierten Benzols beobachtet, obwohl es sich hier bei dem $2 v_{\mathrm{CH}}$-ZOBS um einen echten edge state handelt. Hier scheint die bessere anharmonische Kopplung im insgesamt größeren QNS die Einschränkung der Diffusionsrichtung zu kompensieren.

\section{Temperaturabhängigkeit}

Wie in Abschn. 5.1.1 bereits erläutert wurde, mußten die Einzelmessungen an den verschiedenen aromatischen Modellsystemen bei unterschiedlichen Meßtemperaturen im Bereich von $100-145{ }^{\circ} \mathrm{C}$ durchgeführt werden. Ein Vergleich der transienten Absorptionen darf nur erfolgen, wenn ein etwaiger Temperatureffekt auf die Energietransferdynamik quantifiziert werden kann. Aus diesem Grund wurde für das Referenzsystem Benzol eine temperaturabhängige Bestimmung der intramolekularen Umverteilungsgeschwindigkeiten vorgenommen. Die schnelle IVR-Komponente blieb in allen fünf Messungen mit $\tau_{\mathrm{IVR}_{1}}=0.5 \mathrm{ps}$ unverändert. Die Zeitkonstanten $\tau_{\mathrm{IVR}_{2}}$ des langsamen IVR-Schrittes sowie die relativen Amplitude $\mathrm{A}_{\text {inst }}$ sind in Abb. 7.2 zusammengefaßt. Eine monotone Abhängigkeit der IVR-Geschwindigkeit oder der Amplitude von der Temperatur ist nicht festzustellen. Vielmehr fällt die Variation der Zeitkonstanten um den Mittelwert von 50.2 ps so gering aus, daß sie die relativen Zeitskalen der Benzol-BenzolderivatVergleiche nicht ändert. Ähnliches trifft auf die relativen Amplituden $\mathrm{A}_{\text {inst }} \mathrm{zu}$. Innerhalb der Meßgenauigkeit darf also von einer temperaturunabhängigen IVRDynamik gesprochen werden.

Diese Beobachtungen lassen sich mit den der IR-Pump-UV-ProbeSpektroskopie zugrundeliegenden Annahmen durchaus vereinbaren. Bei einer Erwärmung von 100 auf $150^{\circ} \mathrm{C}$ nimmt die Innere Energie des Benzols um weniger als $500 \mathrm{~cm}^{-1}$ zu. Die Temperaturerhöhung äußert sich somit allenfalls in einer er- 

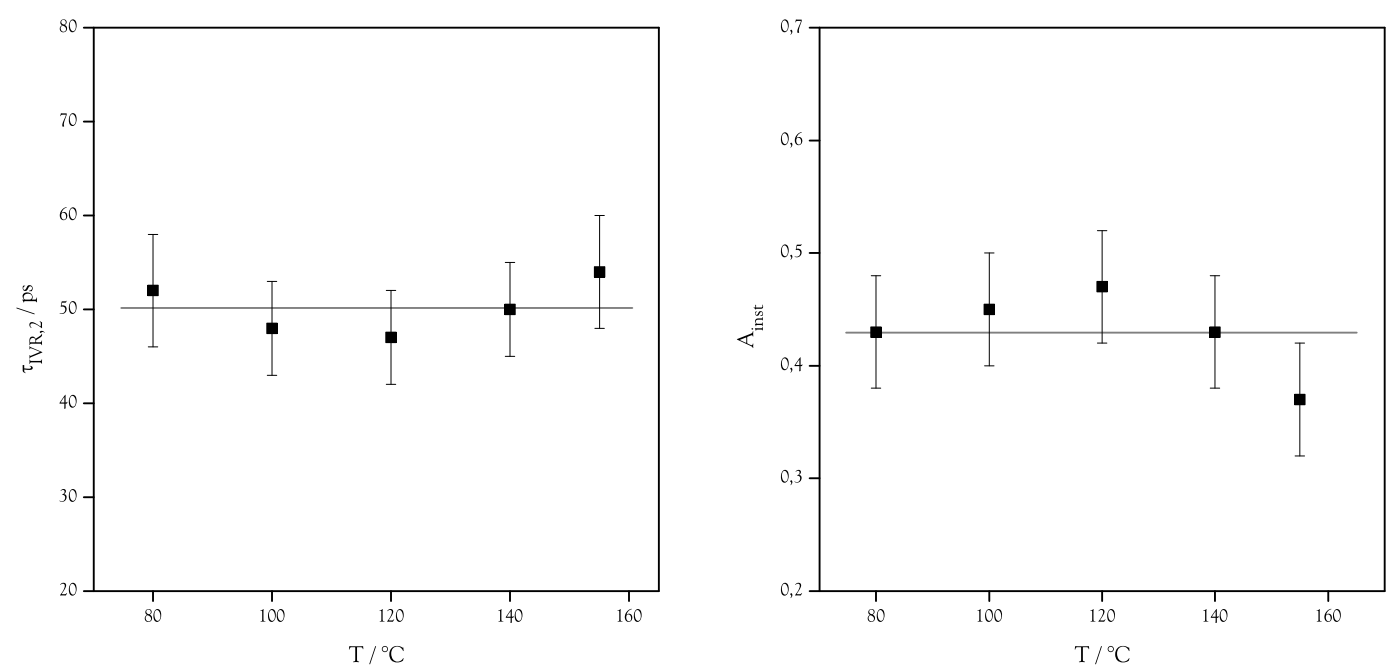

Abbildung 7.2: Temperaturabhängigkeit von $\tau_{\mathrm{IVR}_{2}}$ (links) und $\mathrm{A}_{\text {inst }}$ (rechts) des Benzols

höhten Besetzungswahrscheinlichkeit der niederfrequentesten Normalmoden des Moleküls. Dabei handelt es sich zwar hauptsächlich um FC-aktive Moden, deren Population die Absorption des UV-Probepulses verursacht, allerdings erfolgt die Detektion der Absorptionsänderung als Differenzmessung (s. Abschn. 3.2.5). Die von der thermischen Besetzung verursachte Grundabsorption wird auf diese Weise eliminiert. Detektiert wird lediglich die aus der Umverteilung der Anregungsenergie resultierende zusätzliche Besetzungsänderung der niederfrequenten Moden. Daß die IVR-Dynamik des Benzols sich mit zunehmender Temperatur nicht signifikant ändert, weist somit direkt auf temperaturunabhängige intramolekulare Relaxationskanäle hin.

In der Literatur finden sich bislang erstaunlich wenige Studien zur Temperaturabhängigkeit der IVR-Geschwindigkeit, die zum Vergleich herangezogen werden könnten. Eine Untersuchung von Pate und Mitarbeitern vergleicht die Relaxationsgeschwindigkeiten einfach angeregter acetylenischer CH-Streckschwingungen in ultrakalten Molekularstrahlen und der Raumtemperaturgasphase [106]. Die beobachtete Beschleunigung des IVR-Prozesses um einen Faktor fünf aufgrund der erhöhten Temperatur stellt jedoch allenfalls einen Näherungswert dar, da hier frequenzaufgelöste mit zeitaufgelösten Daten verglichen werden, womit eine eindeutige Korrelation der experimentellen Zeitskalen nicht möglich ist. Eine andere 
Studie von Liu et al. befaßt sich mit der Relaxation von Farbstoffmolekülen im ersten elektronisch angeregten Zustand [5]. Bei einer Temperaturerhöhung um $100^{\circ} \mathrm{C}$ wurde eine Verringerung der IVR-Lebensdauer von 400 auf 300 fs gemessen. Eine Übertragung dieses Ergebnisses auf die Systeme der vorliegenden Arbeit ist allerdings insofern ausgeschlossen, als daß es sich um Experimente in kondensierter Phase handelt, in denen der Einfluß der Lösungsmittelumgebung auf die IVR-Dynamik ungeklärt ist.

\subsubsection{Molekulare Symmetrie und IVR}

In der Literatur dokumentierte Studien zum intramolekularen Schwingungsenergietransfer in isolierten Molekülen setzen mehr oder weniger explizit die uneingeschränkte Gültigkeit der Symmetriebedingung aus der Auswahlregel für strahlungslose Übergänge voraus. Diese besagt, daß nur Zustände gleicher Symmetrie miteinander strahlungslos wechselwirken können (s. Abschn. 2.1.1). Die strenge Anwendung dieses Kriteriums schränkt die Anzahl für eine intramolekulare Kopplung zur Verfügung stehenden Zustände je nach Punktgruppe des Moleküls mehr oder weniger stark ein. Die resultierende symmetriereduzierte Zustandsdichte wird im folgenden als $\rho_{\text {sym }}$ bezeichnet. Im statistischen IVR-Regime müßte Fermis Goldene Regel (Gl. (2.9) in Abschn. 2.1.1) dementsprechend zu

$$
k_{\mathrm{FGR}}=\frac{2 \pi}{\hbar} \rho_{\mathrm{sym}}\langle V\rangle^{2}
$$

umformuliert werden. Für zwei strukturell eng verwandte Moleküle $a$ und $b$, die sich lediglich in ihrer Symmetrie unterscheiden, aber ähnliche Gesamtzustandsdichten besitzen, sagt Gl. (7.2) eine Änderung der IVR-Geschwindigkeit um den Faktor $\rho_{\mathrm{sym}, a} / \rho_{\mathrm{sym}, b}$ voraus, solange das mittlere Kopplungsmatrixelement $\langle V\rangle$ konstant bleibt.

\section{Benzol/Benzol-d}

Mit den Molekülen Benzol und Benzol- $\mathrm{d}_{1}$ steht ein Modellsystem zur Verfügung, an dem diese Vorhersage auf elegante Weise überprüft werden kann. Wie bereits in Abschn. 4.2 ausgeführt, stellt die Monodeuterierung einen äußerst geringen Eingriff in die chemische Natur des Benzols dar. Die Absolutwerte der meisten Normalmodenfrequenzen ändern sich durch die Substitution kaum. Dementsprechend weicht auch die Gesamtzustandsdichte des $\mathrm{C}_{6} \mathrm{H}_{5} \mathrm{D}$ von der des $\mathrm{C}_{6} \mathrm{H}_{6}$ nur 
7.1 IVR in quasi-isolierten Molekülen

\begin{tabular}{|r|cc|}
\hline & Benzol & Benzol-d $_{1}$ \\
\hline \hline Punktgruppe & $\mathrm{D}_{6 \mathrm{~h}}$ & $\mathrm{C}_{2 \mathrm{v}}$ \\
Anzahl irreduzibler Darst. & 12 & 4 \\
Symmetrie des ZOBS & $\mathrm{E}_{1 \mathrm{u}}$ & $\mathrm{A}_{1},\left(\mathrm{~B}_{1}, \mathrm{~B}_{2}\right)$ \\
$\rho / \mathrm{cm}$ & 1600 & 2000 \\
$\rho_{\mathrm{sym}} / \mathrm{cm}$ & 133 & 500 (Fall 1) bzw. 1500 (Fall 2) \\
\hline
\end{tabular}

Tabelle 7.1: Symmetriereduzierte Zustandsdichten von Benzol und Benzol- $\mathrm{d}_{1}$ (berechnet für $6000 \mathrm{~cm}^{-1}$ )

um $20 \%$ ab. Die symmetriereduzierte Zustandsdichte hingegen nimmt durch die Monodeuterierung sehr stark zu, was in Tab. 7.1 veranschaulicht ist. Bei Benzol darf nur 1/12 der Gesamtzustandsdichte mit dem ZOBS koppeln. Durch die Deuterierung ändert sich die Punktgruppe des Moleküls von $\mathrm{D}_{6 \mathrm{~h}}$ auf $\mathrm{C}_{2 \mathrm{v}}$, womit die Zahl irreduzibler Darstellungen von zwölf auf vier sinkt. Gleichzeitig erlauben die optischen Auswahlregeln nun Übergänge zu allen Zuständen, die eine andere Symmetrie als $\mathrm{A}_{2}$ besitzen.

Im nIR-Absorptionsspektrum des Deuterobenzols wird die intensivste Absorptionsbande um $6000 \mathrm{~cm}^{-1}$ eindeutig von ersten Obertönen der $\mathrm{CH}$ Streckschwingungen verursacht, die totalsymmetrisch sind. Der ZOBS besäße demnach primär $\mathrm{A}_{1}$-Symmetrie. Somit ständen dem ZOBS 1/4 aller Zustände in $\rho$ als Kopplungspartner zur Verfügung (Fall 1 in Tab. 7.1). Allerdings ist der ZOBS nur eine phänomenologische Beschreibung des nicht-stationären Schwingungszustands bei $t=0$ (s. S. 9); in der Realität erzeugt der ultrakurze Laserpuls eine Superposition von hellen Zuständen, die sehr wahrscheinlich auch $\mathrm{B}_{1}$ - und $\mathrm{B}_{2}$-Komponenten enthält. Unter der Annahme, daß der superponierte angeregte Zustand simultan an alle beteiligten Symmetriespezies koppeln darf, wären 3/4 der Gesamtzustandsdichte für den Energietransfer zugänglich (Fall 2) ${ }^{1}$. Effektiv ergäbe sich hieraus nach Fermis Goldener Regel eine IVR-Beschleunigung infolge der Symmetriereduktion von

$$
\frac{\rho_{\text {sym }, \mathrm{D}}}{\rho_{\text {sym }, \mathrm{H}}} \approx 4 \quad\left(\text { Fall 1) bzw. } \quad \frac{\rho_{\text {sym }, \mathrm{D}}}{\rho_{\text {sym }, \mathrm{H}}} \approx 11 \quad(\text { Fall } 2)\right.
$$

\footnotetext{
${ }^{1}$ Dürfte hingegen jeder an der Superposition beteiligte Zustand nur innerhalb seiner eigenen Symmetrie koppeln, läge ebenfalls Fall 1 vor.
} 
Die im Rahmen dieser Arbeit experimentell ermittelten IVR-Zeitkonstanten können diese Vorhersage für Fall 2 nicht bestätigen. Sowohl für die Obertonanregung mit ca. $6000 \mathrm{~cm}^{-1}$ als auch für die Kombinationsanregung mit ca. $4500 \mathrm{~cm}^{-1}$ ist der IVR-Prozess in Benzol- $\mathrm{d}_{1}$ nur um einen Faktor 3-4 schneller als in Benzol.

An dieser Stelle muß vor einer vorschnellen Interpretation der Übereinstimmung mit Fall 1 gewarnt werden. Mehrere Faktoren sprechen dafür, daß diese Übereinstimmung eher zufälliger Natur ist:

- Die Beschleunigungen in (7.3) ergeben sich aus einer konsequenten Anwendung von Fermis Goldener Regel. Diese gilt allerdings nur für eine statistische und dementsprechend simultane Umverteilung der Anregungsenergie an allen dunklen Hintergrundzustände. Der multiexponentielle Charakter der IVR-Dynamik beweist jedoch eindeutig, daß IVR weder in Benzol noch in Benzol- $\mathrm{d}_{1}$ statistisch beschrieben werden kann.

- Die Symmetrie hoch angeregter CH-Streckschwingungen des Benzols ist in $\mathrm{D}_{6 \mathrm{~h}}$ nicht immer klar zu definieren, wie daran zu sehen ist, daß für ihre Beschreibung sowohl eine Normalmoden- als auch eine Lokalmodenbasis erfolgreich verwendet werden kann (s. Abschn. 4.1). Es ist nicht auszuschließen, daß in der Realität eine niedrigere Punktgruppe als $\mathrm{D}_{6 \mathrm{~h}}$ vorliegt, wodurch selbst bei strenger Anwendung des Symmetriekriteriums eine größere Anzahl von Zuständen miteinander koppeln dürften.

Ferner treten auch unter den quasi-isolierten Bedingungen der Pump-ProbeMessungen noch vereinzelte intermolekulare Stöße auf $\left(<10^{-2} \mathrm{ps}^{-1}\right)$, welche die Symmetrie des Systems ebenfalls stören können.

- An einer Stelle der Relaxationskaskade muß ein Übergang zwischen verschiedenen Symmetrien stattfinden, da sich auch nach Laseranregung bei $t=\infty$ schließlich ein thermisches Ensemble ausbildet. Falls das Symmetriekriterium überhaupt zu irgendeinem Zeitpunkt streng gilt, dann ist dies in der frühen Phase der Umverteilung am wahrscheinlichsten. Ein Effekt der Monodeuterierung wurde jedoch ausschließlich auf der sekundären, langsamen IVR-Zeitskala beobachtet.

All dies weist darauf hin, daß die Symmetriereduktion bei näherer Betrachtung keine befriedigende Erklärung für die beobachtete IVR-Beschleunigung liefern 
kann. Im folgenden wird daher ein alternativer Erklärungsansatz für den Effekt Monodeuterierung auf die intramolekulare Dynamik vorgestellt.

Die Zustandsdichte des Benzolmoleküls zeigt aufgrund der Entartung der zehn Normalmoden mit $\mathrm{e}_{\mathrm{xx}}$-Symmetrie bei gleichzeitigem Fehlen von sub- $400 \mathrm{~cm}^{-1}$ Schwingungen starke Modulationen (s. Abb. 4.12 auf S. 91). Im Bereich der $2 v_{\mathrm{CH}}$-Anregung schwankt $\rho$ innerhalb weniger $\mathrm{cm}^{-1}$ um bis zu 600 Zustände pro $\mathrm{cm}^{-1}$. Als Konsequenz ist im Zustandsraum eine anisotrope Anordnung der potentiell koppelnden Zustände anzunehmen, die sich in einer Ausbildung von Substrukturen mit hoher Gitterpunktdichte äußert. Erwartungsgemäß sollte innerhalb eines solchen „Zustandsclusters“ die Energieumverteilung effizienter sein als zwischen zwei verschiedenen Clustern, da in letzterem Fall die Dimension des Energiediffusion eingeschränkt wird ${ }^{2}$.

Durch die Substitution eines Wasserstoffatoms durch Deuterium wird die Entartung der Normalmoden aufgehoben. Hierdurch verliert die Zustandsdichte des Moleküls ihre energieabhängige Strukturierung und geht in eine nahezu monoton ansteigende Funktion über. Dies bewirkt eine Reduktion der Anisotropie im QNS, d. h. bei gleichbleibender Gesamtzahl von Gitterpunkten sind diese gleichmäßiger um den ZOBS verteilt. Die Besetzungsdiffusion ist somit nicht mehr primär auf einen Zustandscluster limitiert, sondern kann effektiv in alle Richtungen des QNS erfolgen. Die beschleunigte IVR-Dynamik wäre dann auf die Elimierung der Engpässe zwischen den Substrukturen zurückzuführen.

Diese Hypothese kann anhand des in Abschn. 2.1.4 eingeführten QNSDiffusionsmodells überprüft werden. Es wurden zu diesem Zweck Simulationen des IVR-Prozesses in Benzol und Benzol- $\mathrm{d}_{1}$ nach Kombinationstonanregung mit $4500 \mathrm{~cm}^{-1}$ durchgeführt, in denen sich lediglich die Normalmodenfrequenzen der Moleküle unterschieden. Die Symmetrie der koppelnden Zustände wurde nicht berücksichtigt. In Abschn. 5.3 sind die Ergebnisse dieser Modellrechnungen vorgestellt. Zusammenfassend ist festzustellen, daß sowohl die charakteristischen Merkmale der intramolekularen Benzoldynamik als auch die Beschleunigung dieser Dynamik in Folge der aufgehobenen Entartung vollständig in den Simulationen reproduziert wurden. Dies ist als deutlicher Hinweis zu werten, daß die Änderung der QNS-Struktur eine wichtige Rolle für die experimentell beobachtete IVRBeschleunigung spielt.

\footnotetext{
${ }^{2}$ Es liegt sozusagen ein Engpaß, engl. bottleneck, der Umverteilung vor.
} 
Kapitel 7 Diskussion

Neben der Vorhersage der Pump-Probe-Signale gestatten die QNSSimulationen auch eine Analyse der zugrundeliegenden Relaxationskanäle. Eine solche Analyse wird für Benzol und Benzol-d $d_{1}$ für den Fall der $\left(1 \nu_{4}+\right.$ $1 \nu_{7}$ )-Anregung im folgenden Abschnitt vorgestellt.

\section{QNS-Analyse}

Die Visualisierung der zeitabhängigen Besetzungsdiffusion im 30-dimensionalen QNS erfordert zwangsläufig eine Projektion des Energieflusses auf drei Dimensionen. Eine Möglichkeit zur Darstellung bietet die Matrix p, die sich aus der Lösung des Differentialgleichungssystems Gl. (2.26) ergibt. Sie beinhaltet die zeitabhängigen Besetzungswahrscheinlichkeiten aller $i$ Zustände im betrachteten QNS. Eine Auftragung der $p_{i}(t)$ veranschaulicht folglich den Populationsfluß im Zustandsraum unter Ausblendung der Richtungsinformation.

Im folgenden werden die beiden Simulationen analysiert, mit denen auch die experimentellen Absorptions-Zeit-Profile reproduziert wurden (s. Abschn. 5.3.2). Da diese Modellierungen auf einer Anregungsenergiebreite von $\Delta E= \pm 5 \mathrm{~cm}^{-1}$ basieren, besteht der QNS des Benzols aus 1908 und der des deuterierten Benzols aus 2231 Schwingungszuständen. Die Zustände wurden nach aufsteigender Energie sortiert und mit ganzzahligen Indizes versehen. Zustände gleicher Energie sind nach zunehmender Zahl von Schwingungsquanten in den drei niederfrequentesten Moden sortiert. Die Graphen der Abb. 7.3 und 7.4 zeigen die Besetzungsdiffusion für eine Simulationszeit von 10 ps. Es ist zu beachten, daß die Zeitachse nichtlinear skaliert wurde. Die gepunktete Linie markiert in allen vier Graphen $t=1 \mathrm{ps}$. Prinzipiell symbolisieren die Zustandsindizes zusätzlich eine (allerdings nicht linear verknüpfte) Energieskala von $\left(E_{\text {Pump }}-5\right) \mathrm{cm}^{-1}$ bis $\left(E_{\text {Pump }}+5\right) \mathrm{cm}^{-1}$, welche aus Gründen der Übersichtlichkeit nicht abgebildet ist. Auf der vertikalen Achse ist die Besetzungswahrscheinlichkeit des Schwingungszustands aufgetragen. Um die Bevölkerung der Hintergrundzustände hervorzuheben, wurde die vertikale Skala in den unteren Auftragungen um einen Faktor zehn gestreckt.

Die Besetzungsdiffusion im Benzol- bzw. Benzol- $\mathrm{d}_{1}-$ Zustandsraum verläuft auf sehr unterschiedliche Weise, wie im vorhergehenden Abschnitt bereits vermutet wurde. Der obere Graph in Abb. 7.3 offenbart eine ausgeprägte lokale Umverteilung der Anregungsenergie innerhalb der Energieschale des ZOBS (Index 824) während der ersten Pikosekunde. Nur drei Zustände anderer Energie erlangen in dieser Zeit eine Populationswahrscheinlichkeit von mehr als 1.5\%, während 

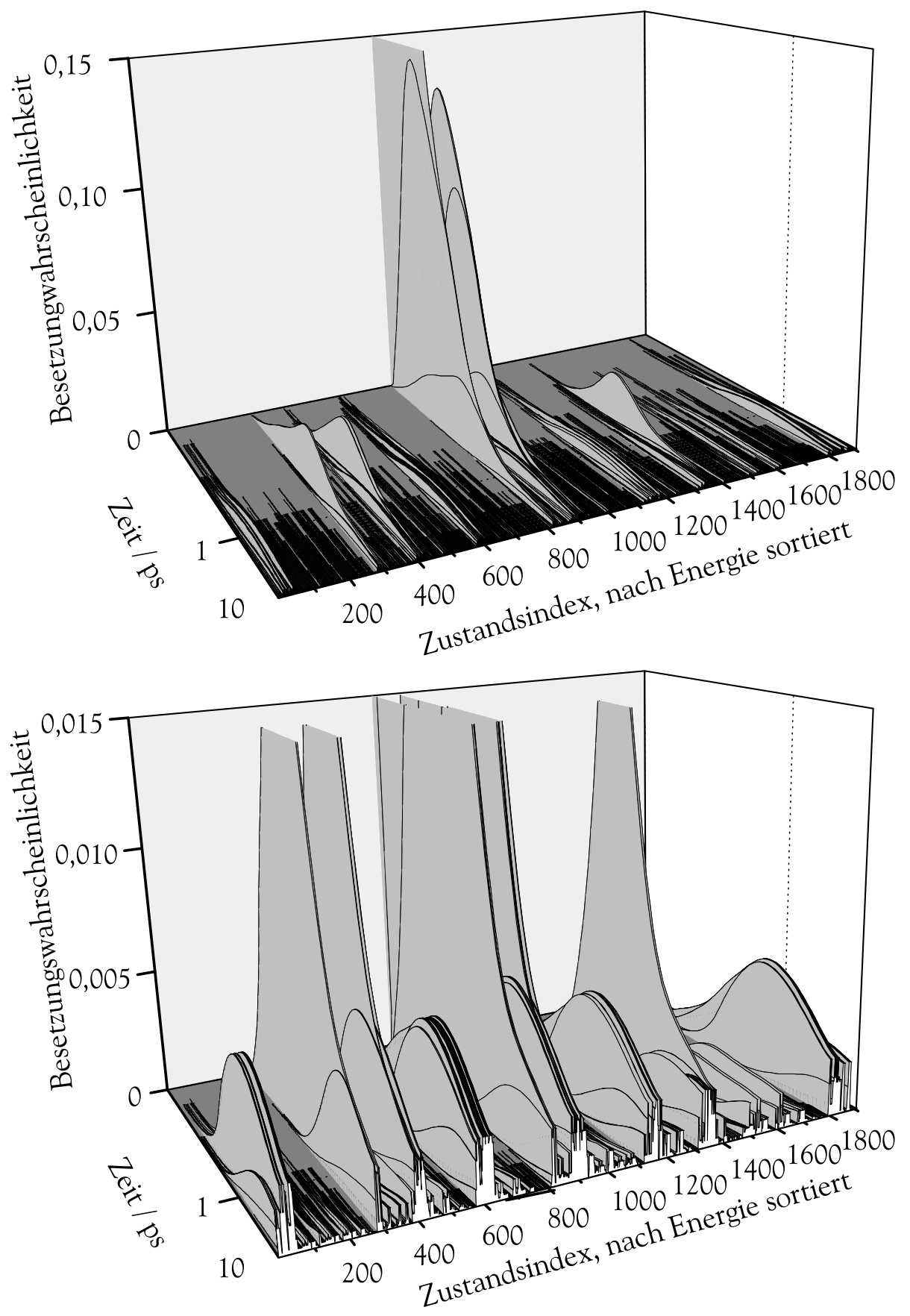

Abbildung 7.3: Zeitaufgelöste Besetzungsdiffusion im QNS des Benzols nach $\left(1 \nu_{4}+1 \nu_{7}\right)$-Anregung mit ca. $4500 \mathrm{~cm}^{-1}$ 

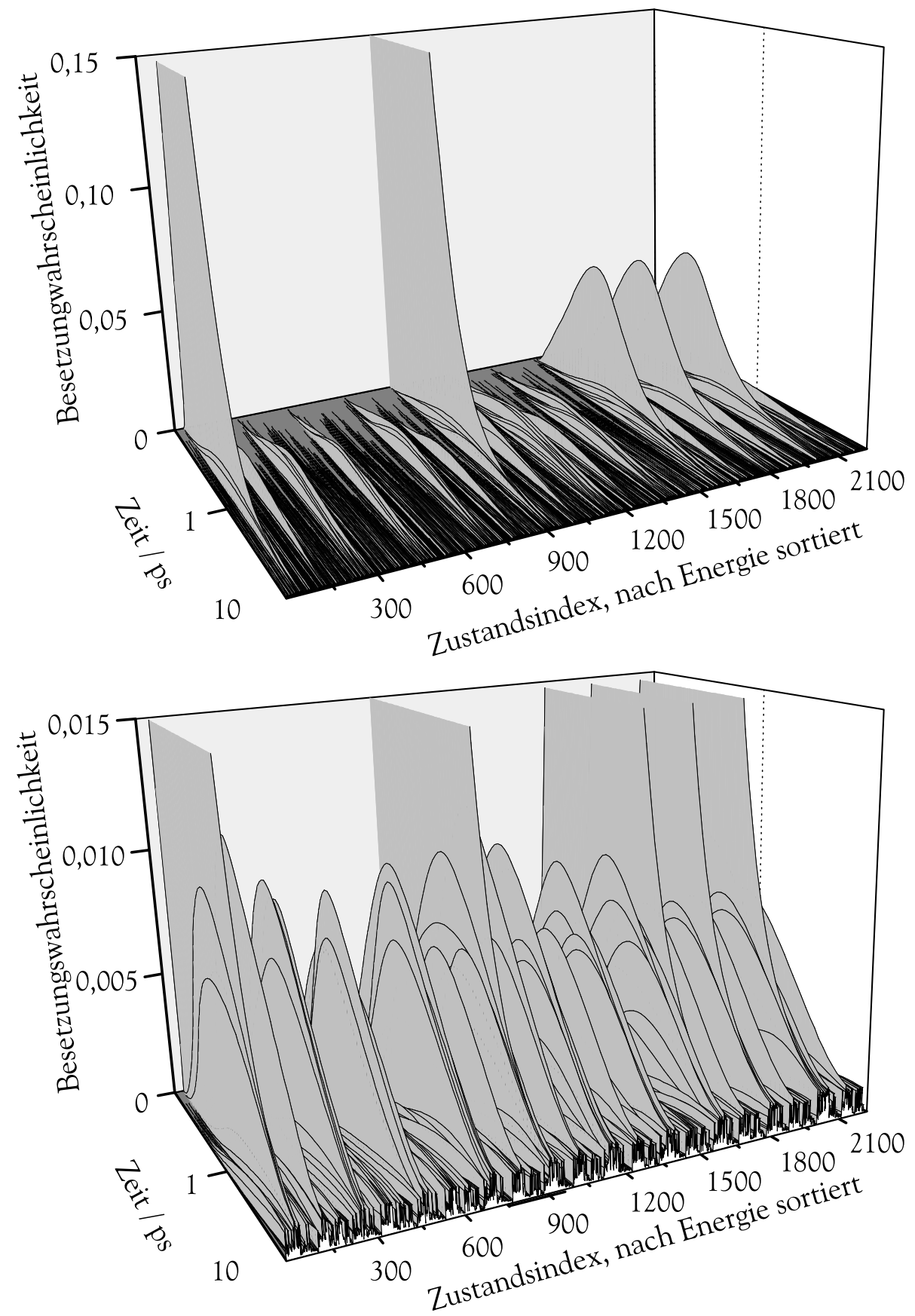

Abbildung 7.4: Zeitaufgelöste Besetzungsdiffusion im QNS des Benzol- $\mathrm{d}_{1}$ nach $\left(1 \nu_{4}+1 \nu_{7}\right)$-Anregung mit ca. $4500 \mathrm{~cm}^{-1}$ 
der Rest des Zustandsraums quasi unbevölkert bleibt. Bei diesen nicht-resonant gekoppelten Zuständen handelt es sich um Kombinationsmoden mit CH-Streck-, CC-Streck- und CCC-Torsionsanteilen. Alle drei haben transienten Charakter und könnten als Schwellenzustände des Energieflusses und damit als erste Stufe des IVR-Stufenmodells bezeichnet werden.

Die Vergrößerung der Darstellung auf max. 1.5\% Besetzungswahrscheinlichkeit im unteren Teil der Abb. 7.3 bestätigt die Annahme einer Clusterung der effizient koppelnden Zustände. Zum einen treten Zustände mit ähnlichem Verlauf von $p$ auch im Hintergrund stark gruppiert auf. Zum anderen sind nach 10 ps erst weniger als ein Drittel aller Zustände des QNS signifikant besetzt, was sich durch die Lokalisierung der Anregungsenergie innerhalb von QNS-Substrukturen erklären läßst.

Im Gegensatz hierzu zeichnet sich das deuterierte Derivat durch eine deutlich isotropere Besetzungsdiffusion aus, wie im oberen Graphen der Abb. $7.4 \mathrm{zu}$ erkennen ist. Eine primäre Umverteilung in energetisch dem ZOBS direkt benachbarte Zustände tritt nicht auf. Stattdessen erlangt in der ersten Pikosekunde eine Vielzahl isotrop verteilter Hintergrundzustände sehr ähnliche transiente Populationswahrscheinlichkeiten von weniger als $1 \%$. Gleichzeitig lassen sich ebenfalls vier nicht-resonante Schwellenzustände zu identifizieren, die denen des Benzols ähneln. Dies suggeriert, daß die enge strukturelle Verwandtschaft der beiden Systeme eine ähnliche Erschließung des QNS in unmittelbarer Umgebung des ZOBS bedingt.

In der vergrößerten Darstellung (Abb. 7.4, unten) fällt zunächst auf, daß nach 1 ps nahezu alle 2231 Zustände eine von Null verschiedene Besetzungswahrscheinlichkeit aufweisen und somit bereits am Energiefluß beteiligt sind. Nach $10 \mathrm{ps}$ liegt eine sehr gleichmäßige Verteilung der Wahrscheinlichkeit auf ca. $80 \%$ der Gesamtzustandsdichte vor. Dies steht in Einklang mit der Annahme einer uneingeschränkten Besetzungsdiffusion in alle Richtungen des multidimensionalen Zustandsraums.

In Hinblick auf die Mechanismen der intramolekularen Energieumverteilung stellt sich die Frage, welche Normalmoden zu einem bestimmten Zeitpunkt der Relaxation am stärksten populiert sind. Es wurden daher in den QNSSimulationen diejenigen Zustände bestimmt, deren Besetzungswahrscheinlichkeit nach einer bzw. zehn Pikosekunden mindestens $0.1 \%$ betrug. Summation über die Besetzungszahlen der dreißig Moden in allen diesen Zuständen liefert eine 


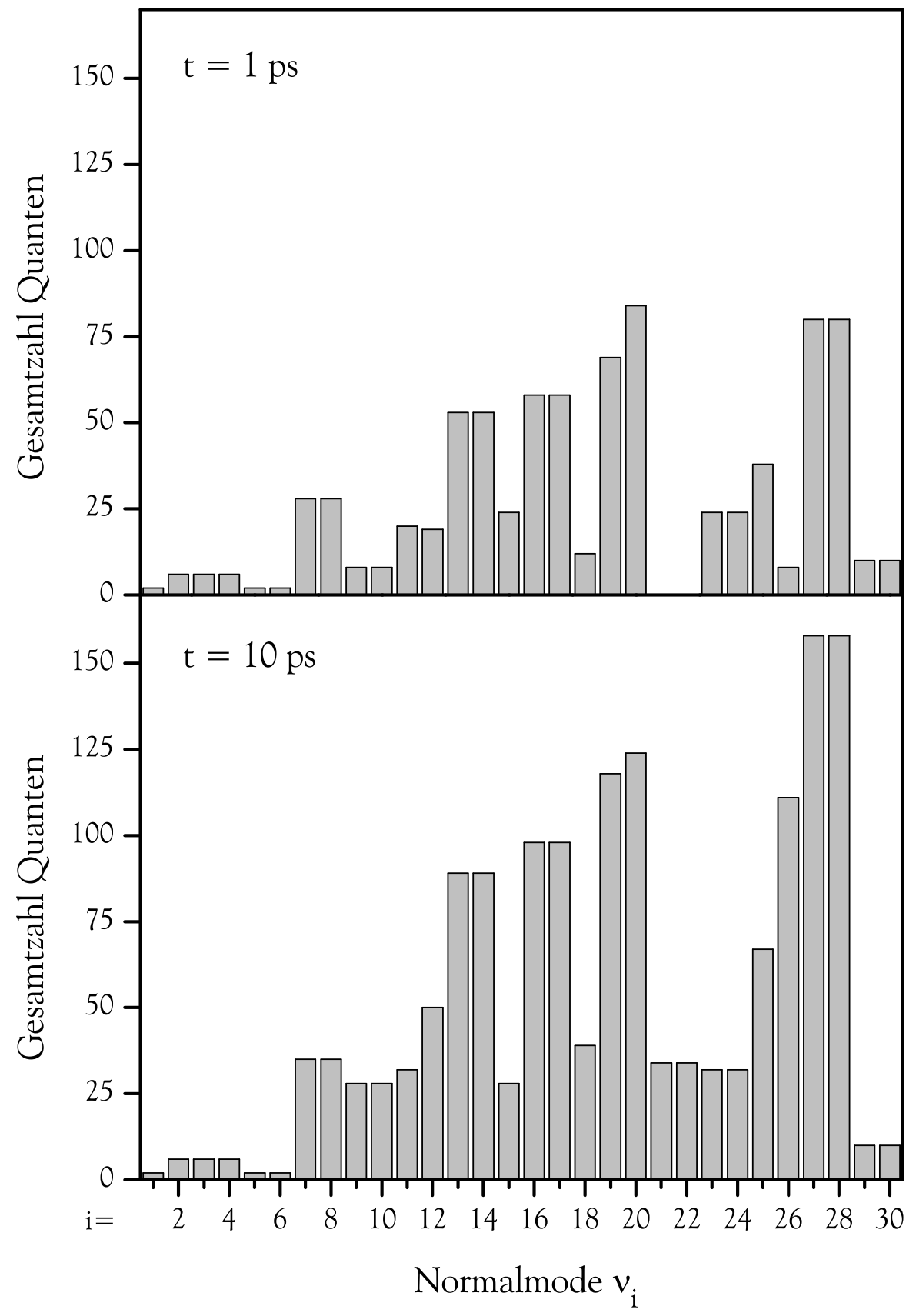

Abbildung 7.5: Statistische Besetzung der Normalmoden des Benzols 1 bzw. 10 ps nach $\left(1 \nu_{4}+1 \nu_{7}\right)$-Anregung mit ca. $4500 \mathrm{~cm}^{-1}$ 


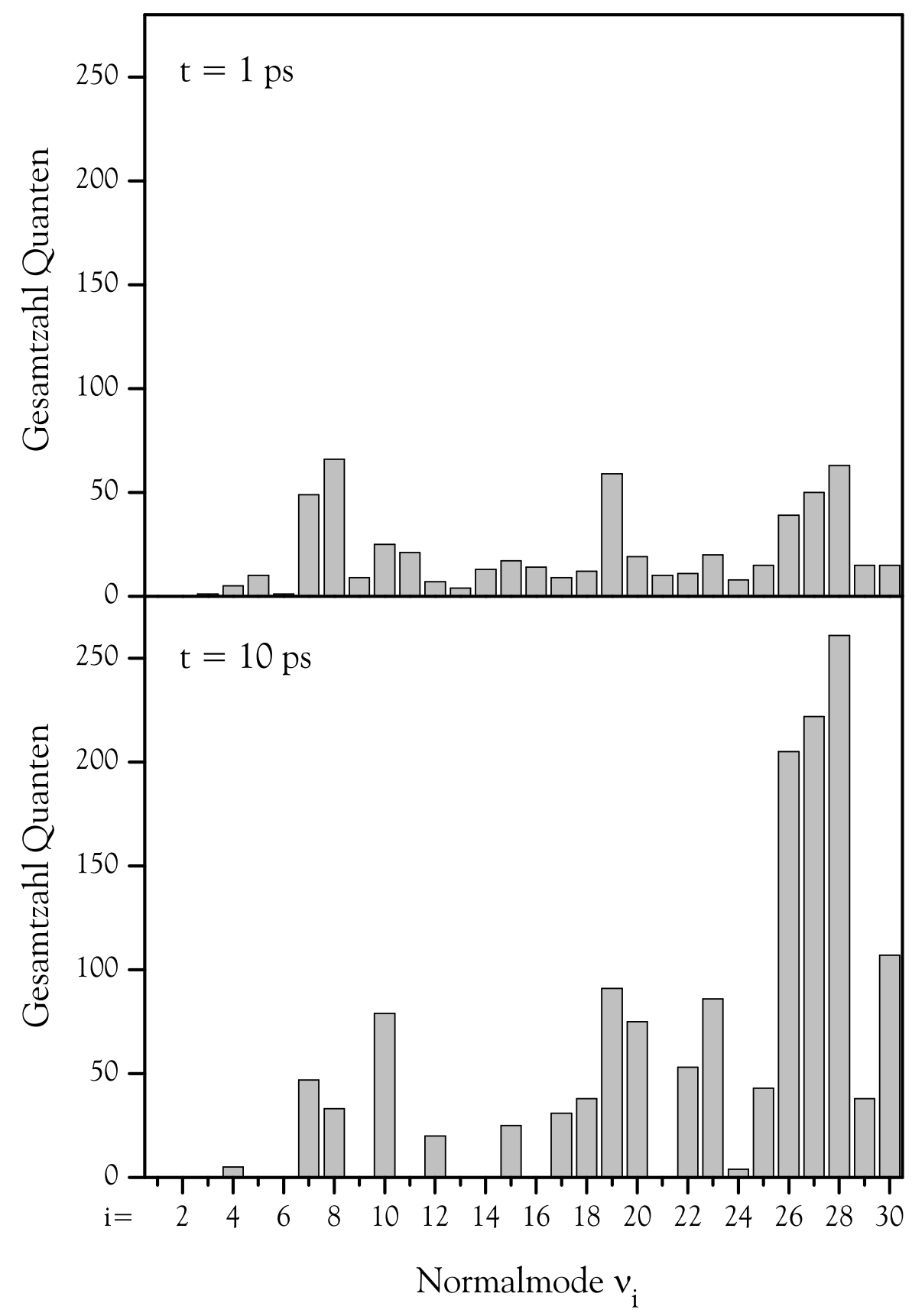

Abbildung 7.6: Statistische Besetzung der Normalmoden des Benzol-d $\mathrm{d}_{1} 1$ bzw. 10 ps nach $\left(1 \nu_{4}+1 \nu_{7}\right)$-Anregung mit ca. $4500 \mathrm{~cm}^{-1}$ 
relative Statistik der Modenbeteiligung am frühen bzw. späten Energieflußs. Für Benzol ist diese Verteilung in Abb. 7.5, für Benzol- $\mathrm{d}_{1}$ in Abb. 7.6 dargestellt. Zur Erleichterung des Vergleichs sind die entarteten Normalmoden des Benzols explizit aufgeführt.

Die Modenstatistiken der beiden Moleküle fallen sehr unterschiedlich aus. In Benzol sind bei $t=1$ ps bereits sechs der zwanzig verschiedenen Normalschwingungen angeregt. Hierbei handelt es sich um die Moden $\nu_{7 / 8}, \nu_{13 / 14}, \nu_{16 / 17}, \nu_{19}$, $\nu_{20}$ und $\nu_{27 / 28}$ (FC-aktiv). Die Gesamtzahl der Quanten in einer Mode nimmt mit sinkender Schwingungsfrequenz zu. Die Form der Verteilung bleibt nach zehn Pikosekunden nahezu unverändert, während lediglich die Gesamtquantenanzahl ansteigt. Dies deutet auf eine Umverteilung der Anregungsenergie zwischen sehr ähnlichen Schwingungszustände hin, wie sie innerhalb eines Zustandsclusters zu erwarten wäre. Im Fall des $\mathrm{C}_{6} \mathrm{H}_{5} \mathrm{D}$ hingegen ist nach 1 ps ein Transfer der Population von den hochfrequenten Moden des ZOBS über die CC-Streckschwingungen $\nu_{7}, \nu_{8}$ und $\nu_{19}$ in die niederfrequenten Ringdeformationsschwingungen erkennbar. Nach 10 ps besitzt die Verteilung ein deutliches Maximum bei den niederfrequenten Moden, während hochfrequente Schwingungen kaum noch populiert sind. Der nach einer bzw. zehn Pikosekunden bevölkerte Teil der Zustandsdichte beinhaltet demzufolge eher unterschiedliche Schwingungszustände, was für eine weitläufige Diffusion der Anfangsbesetzung im QNS spricht.

In beiden Fällen erscheint die Population der niederfrequentesten Normalmoden $\nu_{29}$ und $\nu_{30}$ am Ende der Simulationszeit zunächst erstaunlich niedrig. Hier ist jedoch zu beachten, daß die Summe der Besetzungswahrscheinlichkeiten stets eins betragen muß. Zu unendlich langen Zeiten ist diese statistisch auf ca. 2000 Zustände verteilt, welche deswegen unter die 0.1\%-Grenze fallen und somit in den abgebildeten Verteilungen fehlen.

\section{Fazit zur Isotopensubstitution}

In der Realität wird die Dynamik der intramolekularen Energieumverteilung höchstwahrscheinlich durch eine Kombination aller in diesem Abschnitt vorgestellter Faktoren - Symmetrie, Entartung der Normalmoden, Strukturierung des QNS - beeinflußt. Bemerkenswerterweise kann in Simulationen die experimentelle Beobachtung, daß die Monodeuterierung des Benzols eine IVR-Beschleunigung um einen Faktor 3-4 bewirkt, jedoch bereits durch die bloße Aufhebung der Schwingungsentartung und damit verbundene Isotropisierung des QNS zufrieden- 
7.1 IVR in quasi-isolierten Molekülen

stellend reproduziert werden. Dies darf als deutlicher Hinweis bewertet werden, daß unter den möglichen Einflüssen die Struktur des Schwingungszustandsraums eine herausragende Rolle einnimmt.

Untersuchungen, die sich explizit mit dem Einfluß der molekularen Symmetrie auf den IVR-Prozess befasse, existieren in der Literatur nur wenige. Hochaufgelöste Messungen in der Frequenzdomäne an den Modellsystemen Anthracen/Anthracen-9- $\mathrm{d}_{1}$ [107] und ${ }^{12} \mathrm{C}_{6} \mathrm{H}_{6} /{ }^{13} \mathrm{C}^{12} \mathrm{C}_{5} \mathrm{H}_{6}$ [100] konnten nur qualitativ belegen, daß ein beschleunigender Effekt der Isotopensubstitution vorliegt. Eine quantitative Einstufung des Phänomens fehlte bislang völlig. Die vorgestellte zeitaufgelöste Studie an Benzol/Benzol- $\mathrm{d}_{1}$ ist somit in ihrer Art zur Zeit einzigartig.

\section{Chlorbenzol}

Der Austausch eines Wasserstoffatoms in Benzol durch ein Chloratom bewirkt ebenfalls eine Reduktion der molekularen Symmetrie von $\mathrm{D}_{6 \mathrm{~h}}$ auf $\mathrm{C}_{2 \mathrm{v}}$. Im PumpProbe-Experiment äußert sich der Effekt der Monochlorierung durch eine Verkürzung der zweiten IVR-Zeitskala auf $6.5 \pm 0.8 \mathrm{ps}$. Somit bewirkt die Einführung eines Chloratoms als „monoatomare Seitenkette“ eine IVR-Beschleunigung um einen Faktor 7-8 im Vergleich zu Benzol und um einen Faktor 2 verglichen mit Benzol-d $\mathrm{d}_{1}$.

Da es sich um eine Schweratomsubstitution handelt, stellt sie einen beträchtlichen Eingriff in die chemische und spektroskopische Natur des Moleküls dar. Bei unveränderter Gesamtzahl von Schwingungsfreiheitsgraden wird die Frequenz einiger Fundamentalschwingungen abgesenkt (s. Abschn. 4.3), womit zwangsläufig eine Zunahme der Schwingungszustandsdichte einhergeht. Zwar beweist die multiexponentielle Dynamik des Chlorbenzols die nicht-statistische Natur der Energieumverteilung, doch steigt mit der Gesamtzahl dunkler Zustände auch die Wahrscheinlichkeit günstiger anharmonischer Kopplungen mit dem ZOBS. Folglich bietet für die beiden isosymmetrischen Modellsysteme Benzol- $\mathrm{d}_{1}$ und Chlorbenzol die vergrößerte Zahl von Hintergrundzuständen die plausibelste Erklärung für die beobachtete IVR-Beschleunigung.

An dieser Stelle ist eine Bemerkung zum in der Literatur als „Schweratomeffekt" bezeichneten Phänomen vonnöten. Dieser Begriff wurde in Zusammenhang mit der Studie zur Relaxation CH-angeregter terminalen Acetylene des Typs $\left(\mathrm{H}_{3} \mathrm{C}\right)_{3} \mathrm{X}-\mathrm{CCH}(\mathrm{X}=\mathrm{C}, \mathrm{Si}, \mathrm{Sn})$ geprägt $[25,26]$. Er bezeichnet die Verlangsamung 
der intramolekularen Energieumverteilung bei Substitution des X-Atoms durch schwerere Elemente der gleichen Gruppe. Der scheinbare Widerspruch zur oben beschriebenen Beschleunigung durch Monochlorierung hebt sich auf, wenn die Position der Substitution verglichen wird. In der Acetylenstudie handelt es sich um einen Austausch von Gerüstatomen, die zwischen angeregtem und abgefragtem Chromophor gebunden sind. Ein effektiver Energiefluß durch das lineare Molekül ist an eine Auslenkung des X-Atoms aus der Ruhelage gekoppelt, die mit zunehmender Atommasse schwieriger wird. Die Substitution am Benzol betrifft hingegen die Seitenkette. Die C-Cl-Bindung ist keine Verbrückung zwischen verschiedenen Untereinheiten des Moleküls und stellt dementsprechend auch keinen zwingenden Kanal für den Energiefluß dar. Vielmehr sind die niederfrequenten C-Cl-Schwingungen eher an Zuständen, die am Ende der Relaxationskaskade bevölkert werden, beteiligt. In Zukunft sollte dementsprechend zwischen Gerüstund Seitenketten-Schweratomeffekten unterschieden werden.

\subsubsection{Interne Rotation und IVR}

\section{Toluol}

Diese Arbeit liefert die ersten Ergebnisse zur Obertonrelaxation reinen Toluols in der Gasphase. Es wurden zwei Zeitkonstanten von 0.8 bzw. $8.2 \pm 0.8$ ps für die intramolekulare Relaxation bestimmt. Der relative Anteil der schnellen Komponente an der gesamten IVR-Dynamik beträgt $30 \pm 5 \%$. Mit diesen Daten ist es erstmals möglich, die Zeitskalen und Mechanismen des IVR-Prozesses in isoliertem Toluol und Benzol zu vergleichen [97].

Die erfolgreiche biexponentielle IVR-Modellierung der experimentellen Daten zeigt, daß auch in Toluol ein sequentieller Energiefluß mit mindestens zwei IVRSchritten stattfindet. Somit versagt auch hier die statistische Beschreibung der Energieumverteilung. Der quasi-instantane Signalanstieg ist in diesem Molekül etwas schwächer ausgeprägt, wie dem geringeren $\mathrm{A}_{\text {inst }}$-Wert $\mathrm{zu}$ entnehmen ist. Dies ist möglicherweise eine Konsequenz der unterschiedlichen FC-Faktoren der beteiligten UV-aktiven Normalmoden bzw. der größeren Probewellenlänge.

Verglichen mit dem unsubstituierten Referenzsystem Benzol ist die zweite IVRKomponente des Toluols um einen Faktor 5-6 beschleunigt. Gleichzeitig steigt durch die Einführung der Methylgruppe in das Molekül die Gesamtzustandsdichte um das 33fache an, wobei allein $30 \%$ des Zuwachses auf neue Schwin- 
7.1 IVR in quasi-isolierten Molekülen

gungszustände zurückgehen, da das Molekül nun acht zusätzliche Oszillatoren in der Methylgruppe besitzt. Die innere Rotation des $\mathrm{CH}_{3}$-Substituenten addiert hierzu noch eine Vielzahl von isoenergetischen Rotationsschwingungszuständen (s. Abb. 4.12 in Abschn. 4.6). Selbst unter Einbeziehung der Auswahlregeln für Innere Rotations-Schwingungs-Kopplungen, wodurch die effektiv koppelnde Gesamtzustandsdichte etwas reduziert wird [16], fällt die Beschleunigung des zweiten IVR-Schrittes sehr viel geringer aus, als durch bloße Betrachtung der Zustandsdichte zu erwarten wäre.

Es stellt sich somit die Frage, inwiefern die von der Methylgruppe beigetragene zusätzliche Zustandsdichte überhaupt an der intramolekularen Umverteilung der Anregungsenergie beteiligt ist. Aus Gasphasenexperimenten von Nesbitt et al. sowie Lehmann und Mitarbeitern ist bekannt, daß die IVR-Lebensdauer des $\mathrm{CH}-\mathrm{Chromophors} \mathrm{in} \mathrm{substituierten} \mathrm{Acetylenen} \mathrm{von} \mathrm{der} \mathrm{Natur} \mathrm{des} \mathrm{Substituenten}$ kaum beeinflußt wird. Selbst bei einer Zunahme der Gesamtzustandsdichte um $10^{7}$ ändern sich die beobachteten IVR-Geschwindigkeiten um weniger als Größenordnung. Offensichtlich bleibt hier das große Reservoir zusätzlicher Hintergrundzustände, welches der Substituent anbietet, bei der Energieumverteilung ungenutzt, da es nicht an den ZOBS koppelt.

In der IR-Pump-UV-Probe-Spektroskopie, die zur Untersuchung der Toluolrelaxation im Rahmen dieser Arbeit angewandt wurde, ist ein Zugang zur methylischen Zustandsdichte ebenfalls nur indirekt möglich. Schließlich findet die IR-Anregung selektiv in CH-Streckschwingungen des Benzolringes statt, und auch die Abfrage der Dynamik erfolgt über FC-aktive Moden, die ausschließlich zum aromatischen Chromophor gehören. Für den Fall, daß zwischen ZOBS und den Methylzuständen keine effizienten Kopplungskanäle existieren, ist eine „Beobachterrolle" des Methylsubstituenten folglich durchaus vorstellbar.

In der Tat sind in der Literatur theoretische Berechnungen dokumentiert, die auf eine teilweise Entkopplung speziell des Methylsubstituenten von der Schwingungsdynamik des Phenylringes hinweisen. Martens und Reinhardt führten klassische Simulationen zur Dynamik des $p$-Fluortoluols im $\mathrm{S}_{1}$-Zustand aus [108]. Diese ergaben, daß der volldimensionale Schwingungsphasenraum des Systems näherungsweise in zwei Subsysteme aufgeteilt ist. Dem ersten gehören neben dem Methylrotator die niederfrequentesten Phenylringschwingungen an, welche sehr stark und chaotisch mit dem Rotator mischen. Innerhalb dieses Teils des Phasenraums sagen die Autoren eine schnelle und effektive Energieumverteilung 
voraus. Das zweite Subsystem konstituieren die übrigen, hochfrequenten Phenylschwingungen. Diese koppeln lediglich indirekt an den inneren Rotator, woraus eine vergleichsweise langsame IVR-Dynamik resultieren sollte. In einer anderen Studie simulierten Guan und Thompson die Schwingungsdynamik von Toluol mit klassischen Trajektorienrechnungen auf einer ab initio Potentialfläche [109]. Sie verglichen die intramolekularen Relaxationswege nach sechsfacher Anregung der Methyl- bzw. Aryl-CH-Streckschwingungen. Die Mechanismen unterschieden sich grundlegend: Während die methylische $\mathrm{CH}-$ Streckschwingung die Anregungsenergie zunächst an methylische CH-Biegeschwingungen und dann an CC-Streck- und Biegemoden des Rings abgibt, relaxiert die arylische $\mathrm{CH}$-Streckschwingung direkt in die Ringschwingungen, woraus auf eine vergleichsweise ineffiziente Kopplung an die Methylgruppe zu schließen ist.

Mit diesem Erklärungsansatz hebt sich auch der scheinbare Widerspruch zwischen den Ergebnissen dieser Arbeit und der Pionierstudie von Parmenter und Stone [15] zum Einfluß eines inneren Rotators auf den IVR-Prozess auf. In zeitaufgelösten Fluorenszenzexperimenten wurde die Dynamik von $p$-Difluorbenzol und $p$-Fluortoluol im ersten elektronisch angeregten Zustand untersucht und eine Zunahme der IVR-Geschwindigkeit um zwei Größenordnungen beobachtet - ein immenser Effekt im Vergleich zu der oben geschilderten Zunahme um lediglich 5-6. Allerdings betrugen die Überschußenergien in der Fluoreszenz-Studie weniger als $2000 \mathrm{~cm}^{-1}$. Die Anregung beschränkte sich somit auf niederfrequente Ringmoden wie die Wilsonmode 6a. Die genaue Natur des Rotatoreffektes wird noch immer kontrovers diskutiert, jedoch haben die existierenden Modelle,

- die bereits erwähnte Separation des Schwingungsphasenraums nach Martens et al. [108],

- die Änderung der Schwingungsanharmonizität durch Van der WaalsWechselwirkungen zwischen methylischen und orthoständigen arylischen Wasserstoffatomen (,intramolekulare Stöße“) [16], und

- die Verformung des Phenylrings durch periodische Hyperkonjugation mit der rotierenden Methylgruppe [110]

eins gemeinsam: Sie alle sagen eine intensive Kopplung der niederfrequenten Ringmoden an die innere Rotation voraus. Damit stehen sie in Einklang mit Parmenters Beobachtung, ohne die mögliche Beobachterrolle der Methylgruppe bei 
7.1 IVR in quasi-isolierten Molekülen

der Relaxation von obertonangeregten CH-Streckschwingungen des aromatischen Rings auszuschließen.

\section{$\alpha, \alpha, \alpha$-Trifluortoluol}

Mit $\alpha, \alpha, \alpha$-Trifluortoluol stand in dieser Arbeit ein zweites, dem Toluol isomorphes Modellsystem zur Verfügung, um die Auswirkung innerer Rotation auf die Energieumverteilung zu untersuchen. Hier wurde im Vergleich zu Toluol nochmals eine Beschleunigung der zweiten IVR-Komponente um einen Faktor zwei beobachtet. Dieses Phänomen kann durch mehrere Faktoren verursacht worden sein. Zum einen vermögen im fluorierten Derivat theoretisch mehrere CF-Schwingungen [1:1]-Resonanzen mit niederfrequenten Ringmoden auszubilden, wodurch neue Relaxationskanäle niedriger Ordnung geschaffen würden. Zum anderen besitzt Fluor einen 1.2 mal größeren Van der Waals-Radius als Wasserstoff. Im Bild der von Parmenter et al. postulierten intramolekularen Stöße sollten die Wechselwirkungen mit den orthoständigen Wasserstoffatomen somit stärker ausgeprägt sein als bei Toluol. Allerdings eignen sich diese Erklärungsmodelle wie beim unfluorierten Molekül am besten, um eine beschleunigte Relaxation niederfrequenter Moden zu beschreiben.

Als weiterer Faktor muß somit die stark vergrößerte Anzahl von dunklen Rotationsschwingungszuständen bedacht werden. Interessanterweise steht genau wie bei Toluol die Zunahme der IVR-Geschwindigkeit in keiner (linearen) Relation zur 35fachen Zunahme der Zustandsdichte durch die Fluorierung des Methylsubstituenten. Dies weist darauf hin, daß wiederum nicht der gesamte vom Substituenten beigetrage rovibratorische Zustandsraum am intramolekularen Energiefluß teilnimmt. Letzten Endes kann dieser Aspekt nur in ab initio-Berechnungen analog zu [108] eindeutig beleuchtet werden. In Anbetracht der als frei einzustufenden inneren Rotation der Perfluormethylgruppe ist die partielle Entkopplung des Substituenten von der im aromatischen Ring induzierten und abgefragten Schwingungsdynamik jedoch durchaus wahrscheinlich.

\subsubsection{Beurteilung der relativen Substituenteneffekte}

Die beschleunigende Wirkung der vier untersuchten Substituenten auf die zweite IVR-Komponente des Benzols ist in Abb. 7.7 schematisch zusammengefaßst. Eben- 


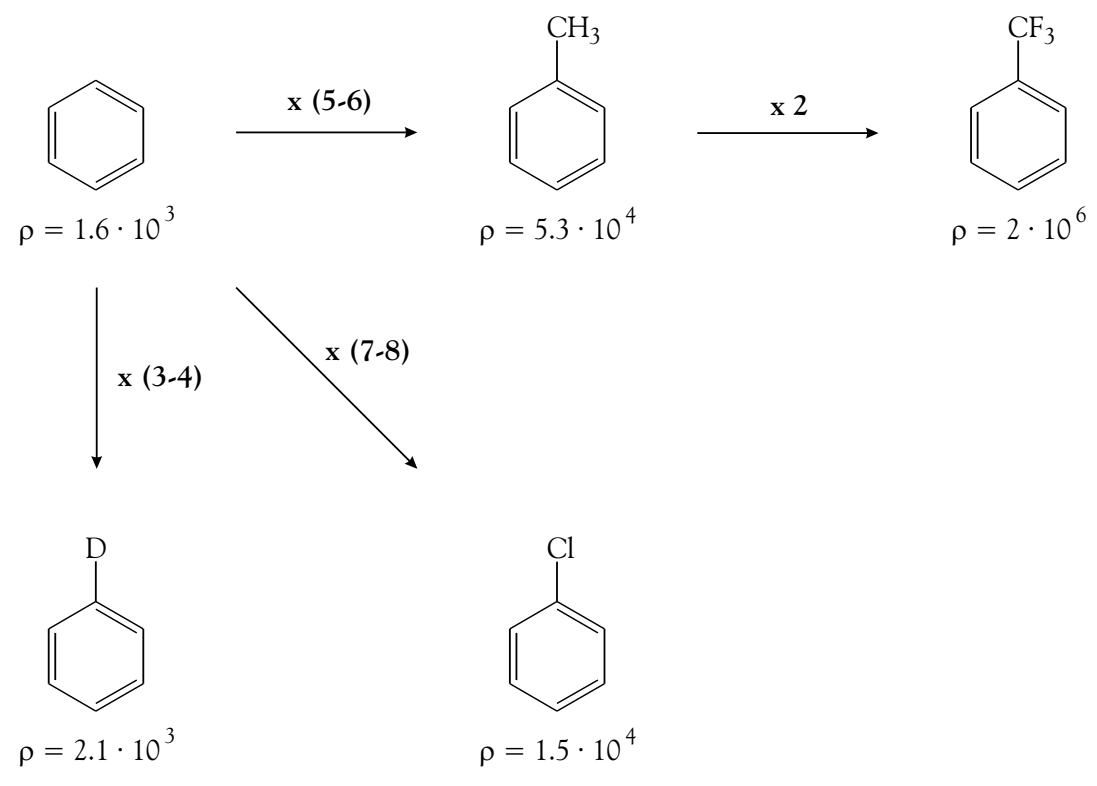

Abbildung 7.7: Relative Beschleunigung der langsamen IVR-Komponente durch chemische Substitution (fett); Zustandsdichten in cm

falls vermerkt ist die korrespondierende Zunahme der Gesamtzustandsdichte. Es liegt offensichtlich keine Fermis Goldener Regel analoge Korrelation zwischen der IVR-Beschleunigung und der Zustandsdichte vor. Besonders deutlich wird dies an den Modellsystemen Chlorbenzol und Toluol, von denen das methylierte System trotz der zusätzlichen Schwingungs- und Rotationszustände der $\mathrm{CH}_{3}$-Gruppe die langsamere IVR-Dynamik aufweist.

Angesichts der Tatsache, daß in den Pump-Probe-Experimenten für alle vier monosubstituierten Derivate der gleiche charakteristisch zweistufige Signalverlauf wie im unsubstituierten Benzol detektiert wurde, liegt es nahe, daß Augenmerk auf die in allen Molekülen identische Struktureinheit, das Phenylfragment $\mathrm{C}_{6} \mathrm{H}_{5}$, $\mathrm{zu}$ richten. In den vorhergehenden Abschnitten wurden bereits einige Anhaltspunkte dafür benannt, daß die Anregung primär innerhalb des Benzolrings delokalisiert wird:

- die Selektion phenylischer Chromophore für Anregung und Abfrage der Schwingungsdynamik; 
7.1 IVR in quasi-isolierten Molekülen

\begin{tabular}{|l|c|cccc|}
\hline & & \multicolumn{4}{|c|}{ Zahl der Kopplungen } \\
Modellsystem & $\boldsymbol{\tau}_{\mathbf{I V R}_{\mathbf{2}}} / \mathbf{p s}$ & $n=3$ & $n=4$ & $n=5$ & $n>5$ \\
\hline \hline Benzol & $48 \pm 5$ & 7 & 356 & 802 & 366500 \\
Benzol-d $_{1}$ & $14 \pm 2$ & 8 & 352 & 946 & 525131 \\
Chlorbenzol & $6.5 \pm 0.8$ & 7 & 325 & 1390 & 3698873 \\
Toluol & $8.2 \pm 0.8$ & 9 & 330 & 1291 & 2281783 \\
$\alpha, \alpha, \alpha$-Trifluortoluol & $3.8 \pm 0.5$ & 5 & 313 & 1393 & 9642584 \\
\hline
\end{tabular}

Tabelle 7.2: Nach Kopplungsordnung sortierte Zustandsdichte für die $2 v_{\mathrm{CH} \text {-streck }}$-Anregung in 30-Moden-Aromaten $\left(\Delta E= \pm 100 \mathrm{~cm}^{-1}\right)$

- die scheinbare Nichtbeteiligung interner Rotationszustände (Vergleich mit Parmenter-Experimenten) und zusätzlicher Substituentenoszillatoren $\left(\mathrm{CH}_{\text {methyl }} / \mathrm{CF}\right)$;

- die Reproduktion des Deuterierungseffektes in QNS-Simulationen mit nicht-entarteten Normalmoden.

Läge tatsächlich eine Entkopplung der Substituentenschwingungen vom benzolischen Zustandsraum vor, wäre die polyatomare (Perfluor-)Methylgruppe aus der Sicht des Phenylrings als Punktmasse zu werten. In diesem Fall wäre der Effekt unterschiedlicher Substituenten auf den IVR-Prozess lediglich auf eine abgestufte Änderung der 30 benzolischen Fundamentalfrequenzen zurückzuführen, da alle Derivate identische Symmetrie besitzen.

Die hohen Zustandsdichten von Chlorbenzol, Toluol und $\alpha, \alpha, \alpha$-Trifluortoluol im Bereich experimenteller Anregungsenergien verhindern eine Überprüfung dieser Annahme durch QNS-Simulationen. Eine für solche Systeme praktikable Alternative zur Zustandsraummodellierung stellt das sogenannte Low Order Coupling Modell (LOCM) dar. Dieses beinhaltet eine Analyse der Gesamtzustandsdichte bezüglich der Ordnung der Kopplung an den ZOBS. Mit Hilfe dieses Modells wurde für gasförmige Acetylene eine Korrelation zwischen der IVR-Lebensdauer und der Anzahl von Kopplungen niedriger Ordnung hergestellt [111], ebenso wie für Alkyliodide in schwach wechselwirkender Lösungsmittelumgebung [112].

Tab. 7.2 präsentiert die Ergebnisse von LOC-Modellierungen für die Modellsysteme dieser Arbeit zusammen mit den experimentell bestimmten IVR,2-Zeiten. Es wurde jeweils ein Startzustand mit $2 v_{\mathrm{CH}^{-}}$Anregung $\left(6000 \mathrm{~cm}^{-1}\right)$ gewählt und 
ein Energieintervall von $\pm 100 \mathrm{~cm}^{-1}$ betrachtet. Toluol und $\alpha, \alpha, \alpha$-Trifluortoluol wurden unter Vernachlässigung der acht Subsituentenschwingungen und der Torsionsmode als 30-Moden-Aromaten simuliert.

Als erstes Ergebnis ist festzuhalten, daß die Anzahl Resonanzen niedriger Ordnung $(\Delta n=3,4)$ sich für die fünf Moleküle kaum unterscheidet. Da diese Resonanzen mit großen Kopplungsmatrixelementen verbunden sind, vermitteln sie höchstwahrscheinlich Übergänge zwischen dem ZOBS und den Zuständen der ersten IVR-Stufe. Die nahezu identischen LOCM-Statistiken dieser Ordnungen suggerieren, daß die frühe IVR-Dynamik der substituierten Derivate der des Benzols gleicht, da die bevölkerten Zustände denen des Benzols entsprechen. Dies würde den auffallend ähnlichen zweistufigen Verlauf der Pump-Probe-Signale erklären.

Die langsame Zeitskala der Energieumverteilung wird hingegen eher von Kopplungen höherer Ordnung $(\Delta n \geq 5)$ diktiert. Abb. 7.8 zeigt anschaulich, daß der Gang der langsamen IVR-Geschwindigkeitskonstante $k_{\mathrm{IVR}_{2}}=1 / \tau_{\mathrm{IVR}_{2}}$ besonders

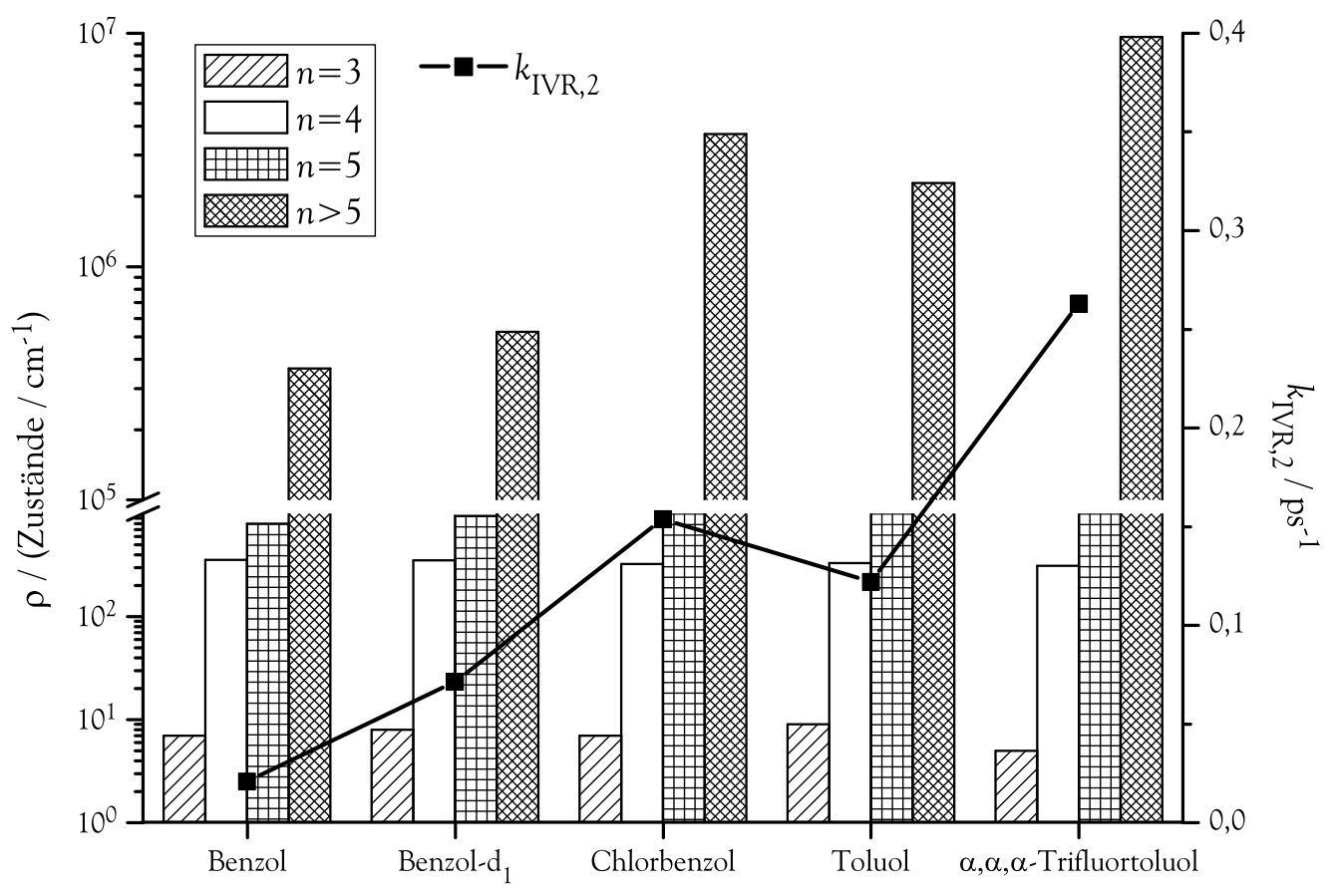

Abbildung 7.8: Korrelation zwischen IVR-Geschwindigkeit und nach Kopplungsordnung sortierter Zustandsdichte für 30-Moden-Aromaten 
mit Resonanzen zu $n>5$ korreliert ist. Eine lineare Skalierung mit der Anzahl dieser Resonanzen liegt allerdings nicht vor. Diese Beobachtungen legen nahe, daß die Masse der Hintergrundzustände indirekt, d. h. über Kopplungen an Zustände früherer IVR-Stufen, die Entvölkerung des ZOBS beeinflußt.

Zusammenfassend bleibt zu konstatieren, daß die experimentell gefundenen Substitutionstrends der IVR-Dynamik mit dem 30-Moden-Modell qualitativ für alle Modellsysteme erklärt werden kann. Dies stellt ein deutliches Indiz dar, daß Substituentenmoden in Aromaten mit frei rotierender polyatomarer Seitenkette eine untergeordnete Rolle in der intramolekularen Schwingungsenergieumverteilung einnehmen.

\subsection{IVR in unterschiedlichen molekularen Umgebungen}

\subsubsection{Dichteabhängigkeit des Energietransfers}

Die ersten Untersuchungen zum dichteabhängigen Energietransfer des Benzols wurden in überkritischem $\mathrm{CO}_{2}$ bei ca. $50^{\circ} \mathrm{C}$ durchgeführt. Die reduzierte Dichte $\rho_{\text {red }}=\rho / \rho_{\mathrm{c}}\left(\rho_{\mathrm{c}}\right.$ : kritische Dichte $)$ wurde dabei zwischen gasphasenähnlichen $\left(\rho_{\text {red }}=0.7\right)$ und lösungsähnlichen Bedingungen $\left(\rho_{\text {red }}=2.1\right)$ variiert. Die IVRDynamik bei $\rho_{\text {red }}=0$ wurde den Experimenten am quasi-isolierten Benzolmolekül entnommen. Auch bei erhöhter Lösungsmitteldichte zeigten die transienten Absorptionsprofile weiterhin den charakteristisch zweistufigen IVR-Verlauf, der auch in der reinen Gasphase gefunden wurde. Während die Zeitkonstante der ultraschnellen IVR-Komponente $\tau_{\mathrm{IVR}_{1}}$ sowie die relative Amplitude $A_{\text {inst }}$ über den gesamten Druckbereich unverändert blieben, wurde die zweite IVR-Zeitskala $\tau_{\mathrm{IVR}_{2}}$ mit steigender $\mathrm{CO}_{2}$-Dichte zunehmend verkürzt. Als erstes Ergebnis läßt sich hieraus festhalten, daß die primäre, ultraschnelle IVR-Komponente dichteunabhängig zu sein scheint.

Die Abhängigkeit des sekundären IVR-Geschwindigkeit sowie der VETGeschwindigkeit von der reduzierten Umgebungsdichte ist in Abb. 7.11 dargestellt. Aufgetragen sind jeweils die Geschwindigkeitskonstanten des Energietransfers, $k=1 / \tau$. In beiden Fällen zeigt die Zunahme der Geschwindigkeitskonstante einen nichtlinearen Verlauf. Von niedrigen zu hohen reduzierten Dichten gehend 

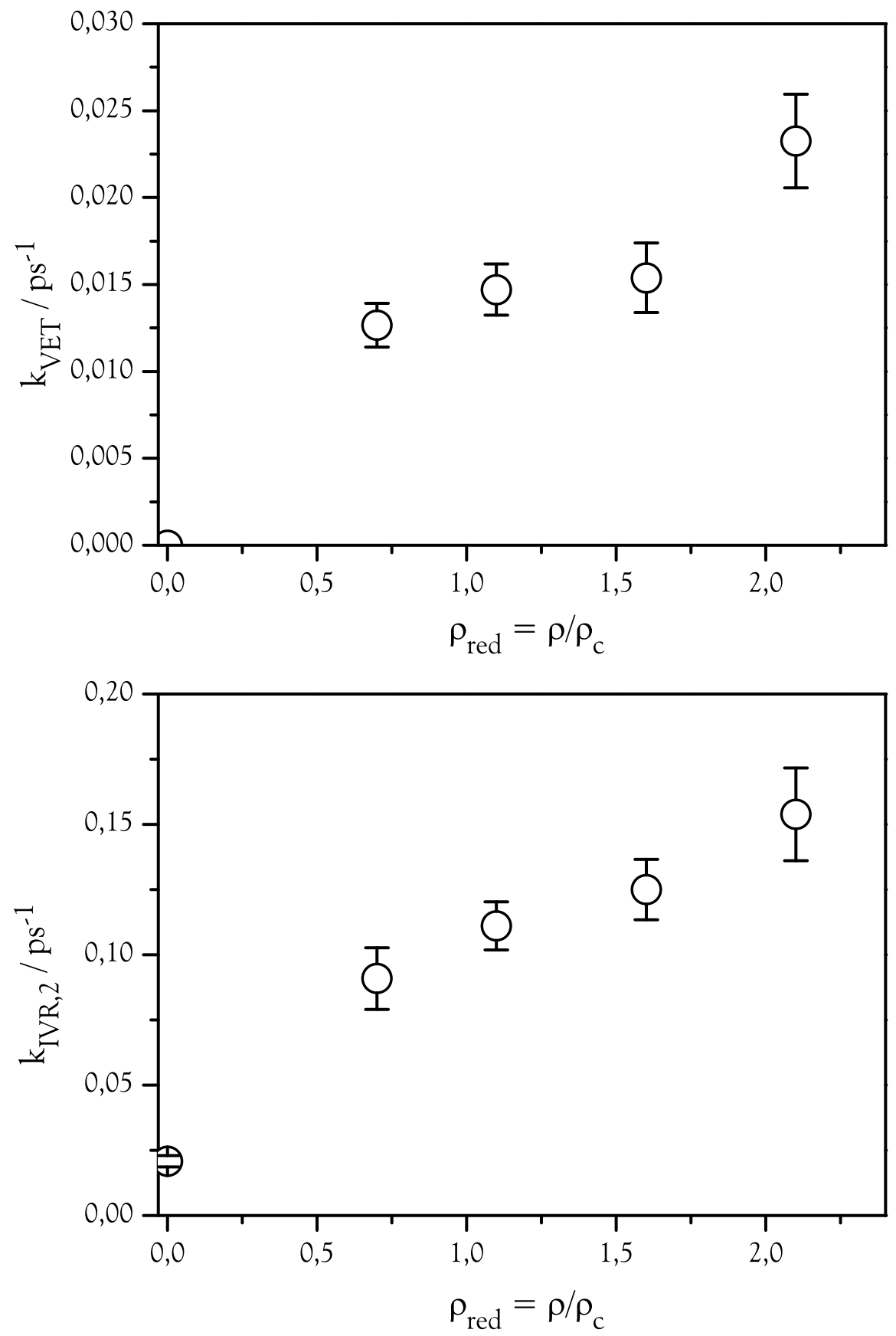

Abbildung 7.9: Dichteabhängigkeit der Geschwindigkeitskonstanten von IVR,2 und VET in überkritischem $\mathrm{CO}_{2}$ 


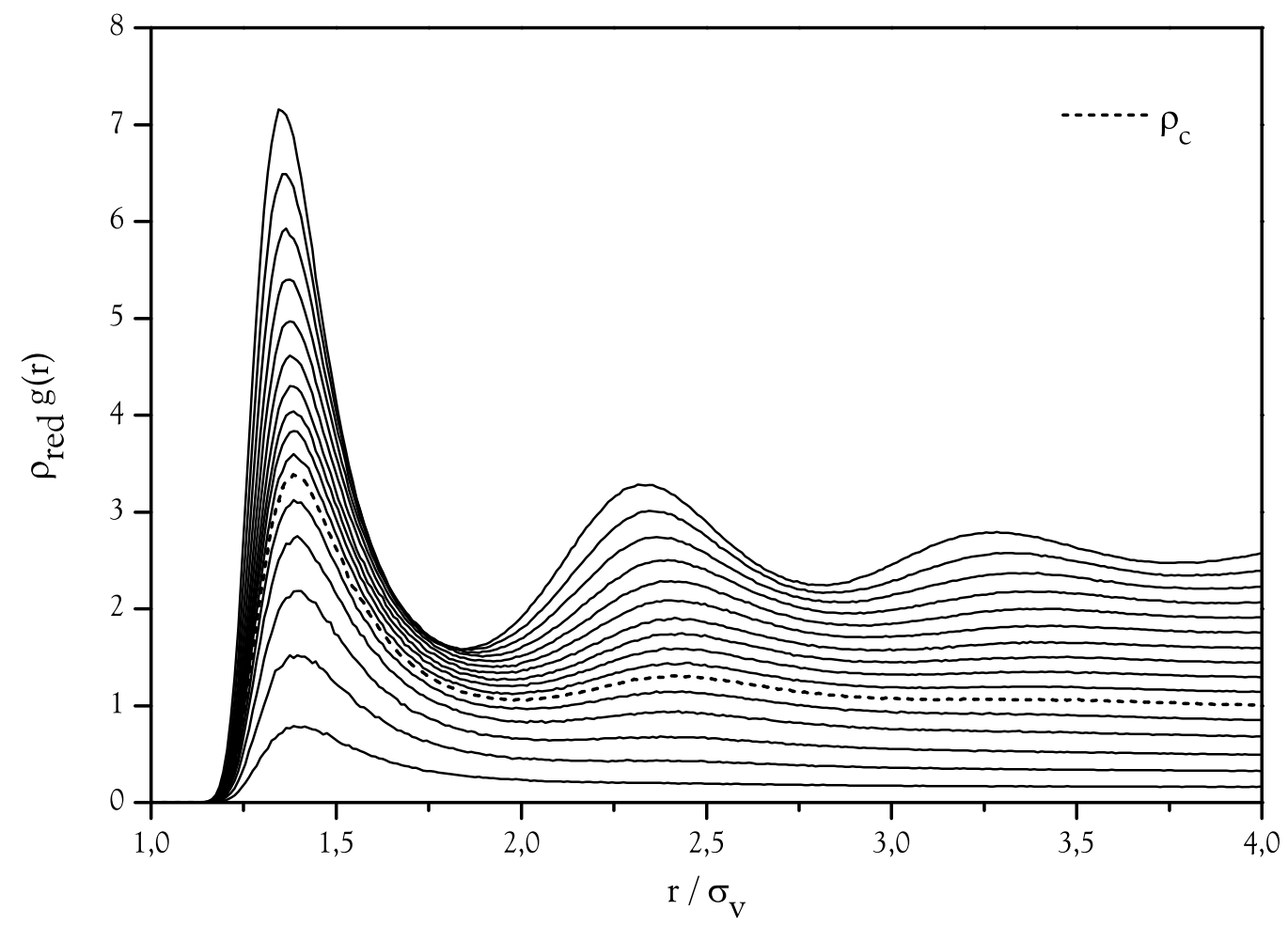

Abbildung 7.10: Radiale Dichteverteilungen um ein attraktives Lennard-JonesTeilchen (Benzol) in einem Lennard-Jones-Fluid $\left(\mathrm{CO}_{2}\right) ; \rho_{\text {red }}=0.16-2.6, \Delta \rho_{\text {red }}=$ 0.16

ist der Anstieg von $k$ zunächst relativ steil, erscheint dann im Bereich der kritischen Dichte etwas geringer ausgeprägt und nimmt anschließend wieder zu.

Dieser S-förmige Verlauf der Dichteabhängigkeit einer physikalischen Größe in überkritischen Fluiden weist oftmals auf lokale Dichteeffekte hin. Schwarzer und Mitarbeiter konnten beispielsweise die Beschleunigung des intermolekularen Energietransfers von Azulen in überkritischen Lösungsmitteln mit dieser Größe korrelieren[34, 35]. Einen Zugang zur lokalen Dichte $\rho_{l o k}$ bieten radiale Verteilungsfunktionen $g(r)$. Im Falle des Benzol- $\mathrm{CO}_{2}$-Systems wurde $g(r)$ mit der Monte Carlo-Methode unter konstanten NVT-Bedingungen berechnet, wobei sowohl Benzol als auch $\mathrm{CO}_{2}$ als Lennard-Jones-Teilchen modelliert wurden. Die Parameter der Simulationen wurden so gewählt, daß sie Benzol als Solvat $u$ und 
$\mathrm{CO}_{2}$ als überkritisches Solvens $v$ repräsentierten; sie sind in Anhang A.3 aufgeführt. Die resultierende Verteilungsfunktionen, multipliziert mit der reduzierten Dichte, zeigt die Abb. 7.10. Die unterste Kurve entspricht $\rho_{\text {red }}=0.16$, die oberste $\rho_{\text {red }}=2.6$. Das Inkrement $\Delta \rho_{\text {red }}$ zwischen einzelnen Kurven beträgt 0.16 . Die Ordinatenskala wurde auf den Lennard-Jones-Durchmesser des Solvates $\sigma_{\mathrm{v}}$ normiert. Bei gleichmäßigem Zuwachs der Gesamtdichte des Systems weist die radiale Verteilungsfunktion je nach Wert von $r$ sehr unterschiedliches Verhalten auf. Während $\rho_{\text {red }} g(r)$ bei großem Abstand vom gelösten Teilchen sehr gleichförmig ansteigt, nimmt das erste Maximum von $g$ bei $1.3 \sigma_{\mathrm{v}}$ von unten nach oben zunächst sehr stark zu. Im Bereich der kritischen Dichte (gestrichelte Linie) hingegen ist die Zunahme weniger ausgeprägt. Erst jenseits der kritischen Dichte ist wieder ein stärkerer Anstieg des Maximums zu erkennen.

Die lokale Dichte $\rho_{\text {lok }}$ ergibt sich direkt aus dem Produkt des ersten Maximums der radialen Verteilungsfunktion $g_{\max }(r)$ mit der reduzierten Dichte. Dementsprechend liefert die Projektion von $g_{\max }(r) \rho_{\text {red }}$ auf $\rho_{\text {red }}$ die Korrelation zwischen lokaler und reduzierter Dichte. Es resultiert die S-förmige Kurve, die in Abb. 7.11 der Auftragung der Geschwindigkeitskonstanten von IVR,2 und VET als durchgezogene Linie hinzugefügt wurde ${ }^{3}$. Aus der sehr guten Übereinstimmung der Modellierung mit den experimentellen Daten folgt, daß $k_{\mathrm{VET}}$ und $k_{\mathrm{IVR}_{2}}$ beide mit der lokalen Dichte von $\mathrm{CO}_{2}$-Molekülen um das angeregte Benzolmolekül skalieren. Im mikroskopischen Bild entspricht die lokale Dichte quasi einer dynamischen Koordinationszahl von Teilchen in der ersten ,Solvathülle“des Benzols, daher ist sie direkt mit der Zahl von Solvat-Solvens-Stößen korreliert. Daraus folgt, daß nicht nur die Geschwindigkeit des intermolekularen Energietransfers, der per se eine Stoßdesaktivierung darstellt, sondern auch die Geschwindigkeit der sekundären IVR-Komponente in einer überkritischen Lösungsmittelumgebung der Stoßzahl proportional ist [96].

\subsubsection{Effekt der Stoßgaseffizienz}

Einige Fragen blieben in der Benzol- $\mathrm{CO}_{2}$-Studie offen, z. B. welche Rolle die Stoßeffizienz der Umgebung für die Beschleunigung der intramolekularen Dynamik spielt, und in welcher Weise die Stoßwechselwirkung mit der Umgebung die Be-

\footnotetext{
${ }^{3}$ In der unteren Auftragung wurde $\rho_{\text {lok }}=0$ so verschoben, daß es die Geschwindigkeitskon-
} stante des isolierten Moleküls erfaßt (graue Linie). 
7.2 IVR in unterschiedlichen molekularen Umgebungen
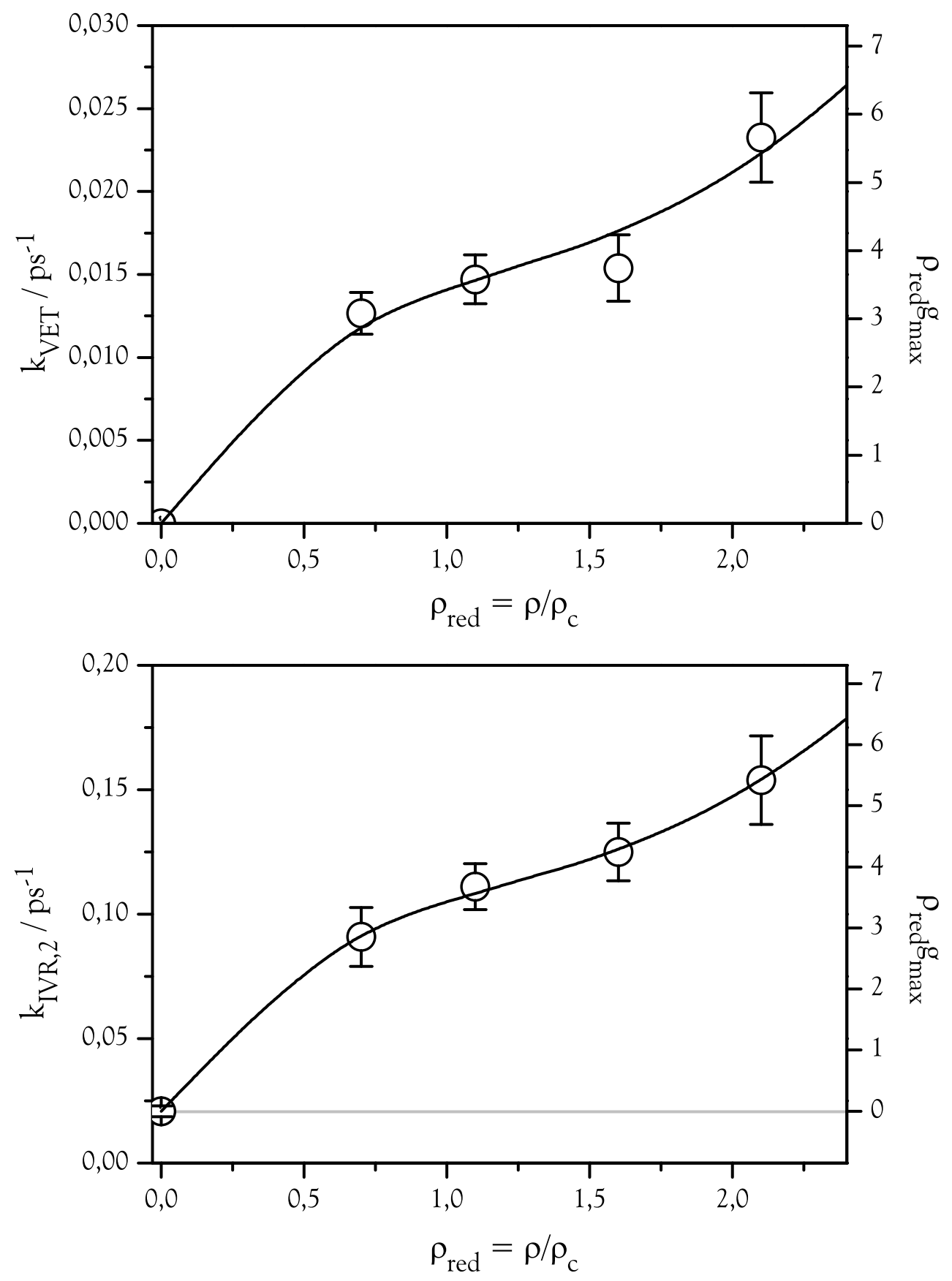

Abbildung 7.11: Korrelation der Geschwindigkeitskonstanten von IVR,2 und VET mit der lokalen $\mathrm{CO}_{2}$-Dichte 
schleunigung bewirkt. Der folgende Abschnitt widmet sich diesen Fragen in einer Erweiterung der dichteabhängigen Studie um verschiedene mono- und polyatomare Badgase.

Die Ergebnisse der Messungen an Benzol in verschiedenen Inertgasumgebungen sind als Zeitkonstanten $\tau_{\text {IVR }}$ und $\tau_{\text {VET }}$ in den Tabellen 6.5-6.7 zusammengefaßst. In dieser Diskussion werden weiterhin die zugehörigen Geschwindigkeitskonstanten $k=\tau^{-1}$ der Prozesse verwendet. Übereinstimmend mit der im vorhergehenden Abschnitt dargelegten Untersuchung nehmen für alle Badgase die langsamen IVR-Geschwindigkeitskonstanten $k_{\mathrm{IVR}_{2}}$ sowie die $k_{\mathrm{VET}}$-Werte mit steigender reduzierter Badgasdichte und folglich mit der Zahl von Benzol-Inertgas-Interaktionen $\mathrm{zu}$.

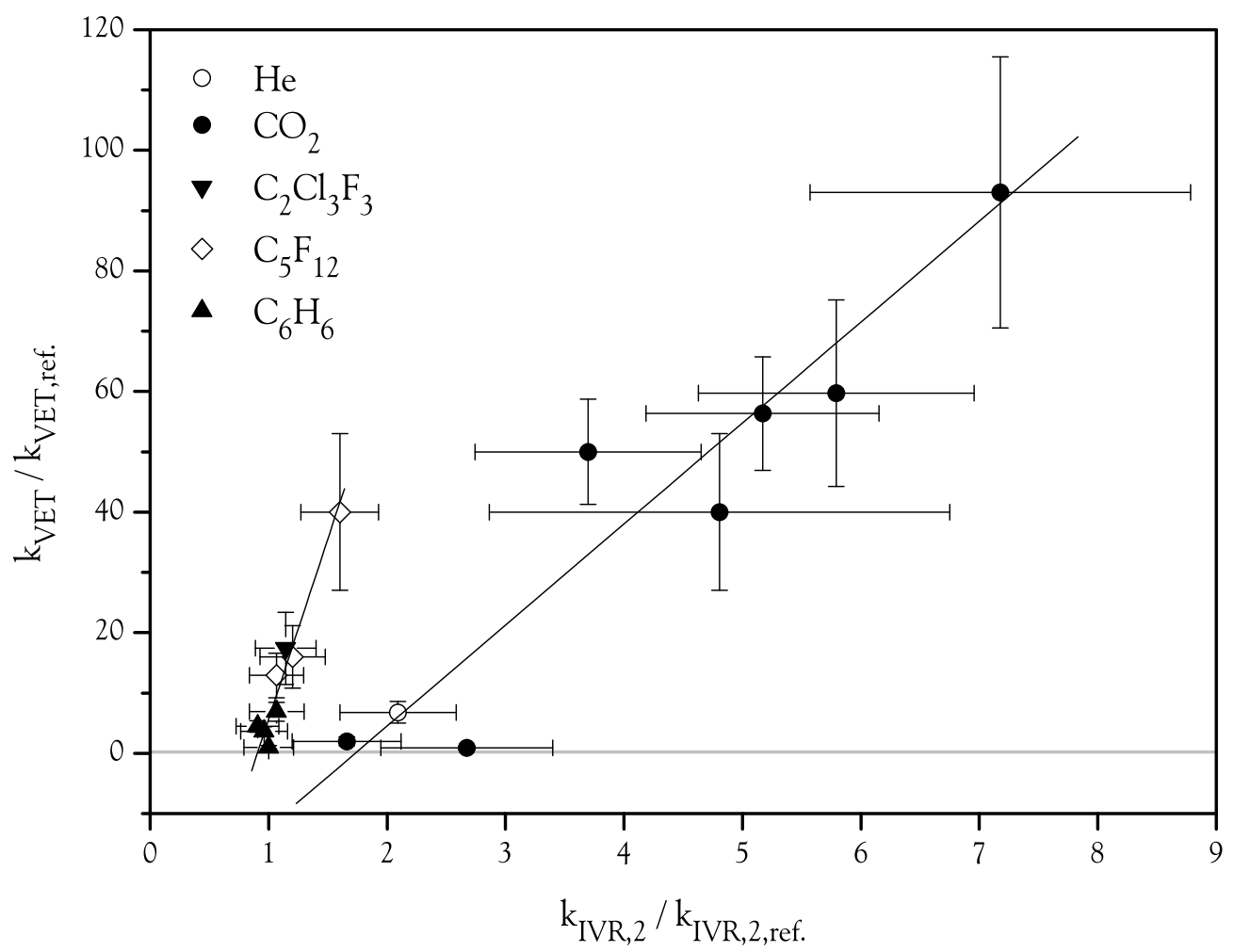

Abbildung 7.12: Korrelation der relativen Beschleunigungen von IVR,2 und VET in verschiedenen Inertgasumgebungen 
Zur Beurteilung der relativen Badgaseffekte dient die Messung an 0.5 bar reinem Benzol als Referenz. Unter diesen Bedingungen beträgt die Lennard-JonesStoßzahl der Benzol-Benzol-Selbststöße weniger als $6 \cdot 10^{-3} \mathrm{ps}^{-1}$, so daß von einer Dynamik quasi-isolierter Moleküle ausgegangen werden kann. Unter dieser Voraussetzung kann die Beschleunigung des Energietransfers durch ein zusätzlich anwesendes Inertgas anhand der Quotienten

$$
\frac{k_{\mathrm{IVR}_{2}}}{k_{\mathrm{IVR}_{2}, \text { ref. }}} \quad \text { bzw. } \quad \frac{k_{\mathrm{VET}}}{k_{\mathrm{VET}, \text { ref. }}}
$$

quantifiziert werden. Derart normierte Geschwindigkeitskonstanten ermöglichen es, die Beschleunigungen von IVR- und VET-Dynamik durch die Inertgasumgebung in Relation zueinander zu setzen. Auf diese Weise kann eine Aussage über die relative Effizienz der durch Wechselwirkung mit der Umgebung bedingten IVR- bzw. VET-Beschleunigung getroffen werden. Abb. 7.12 zeigt die Korrelation der IVR- und VET-Beschleunigungen aller untersuchten Inertgase inklusive der druckabhängigen Benzolmessungen und der Daten aus [96]. Jeder Punkt der Auftragung steht für das aus einer Messung stammende Wertepaar von IVRund VET-Geschwindigkeitskonstanten. Bei Punkten, die im gleichen Badgas aufgenommen wurden, nimmt die reduzierte Dichte des Gases von unten nach oben $\mathrm{zu}$.

Die Auftragung der IVR-VET-Beschleunigungen weist drei auffällige Merkmale auf, die im folgenden einzeln diskutiert werden. Zum einen liegen die zu einem Badgas gehörenden Punkte auf einer Geraden. Dies deutet darauf hin, daß die Beschleunigung des IVR-Prozesses auf die gleiche Weise wie die VETBeschleunigung verursacht wird. Im Falle des intermolekularen Schwingungsenergietransfers ist bekannt, daß Stöße mit den Inertgasmolekülen die Übertragung der Energie vermitteln. Demnach ist sehr wahrscheinlich auch die IVRBeschleunigung auf Stöße mit der Umgebung zurückzuführen. Diese Schlußfolgerung ist mit den Ergebnissen der ersten $\mathrm{CO}_{2}$-Benzol-Studie konsistent, in der eine Proportionalität zwischen der Geschwindigkeit des sekundären IVR-Schrittes und der Solvat-Solvens-Stoßzahl gefunden wurde (s. Abschn. 7.2.1).

Zum zweiten erscheinen die Daten in zwei Gruppen unterteilt: Die zu schweren Badgasen gehörenden Punkte beschreiben eine steilere Gerade als die $\mathrm{CO}_{2^{-}}$und He-Werte. Die Steigungen unterscheiden sich um ca. einen Faktor drei. Daß für unterschiedliche Stoßgase unterschiedliche Steigungen auftreten, bedeutet, daß ein badgasspezifisches Merkmal auf den VET eine andere Auswirkung als auf 


\begin{tabular}{|l|ccc|}
\hline Stoßgas & $\langle-\mathbf{\Delta E}\rangle / \mathbf{c m}^{-1}$ & $\boldsymbol{\sigma} / \AA$ & $\mathbf{M} /\left(\mathbf{g} \mathbf{~ m o l}^{-\mathbf{1}}\right)$ \\
\hline \hline $\mathrm{He}$ & 75 & 2.82 & 4 \\
$\mathrm{Ar}$ & 130 & 3.47 & 40 \\
$\mathrm{CO}_{2}$ & 280 & 3.94 & 44 \\
$\mathrm{C}_{6} \mathrm{H}_{6}$ & 610 & 5.46 & 78 \\
$\mathrm{C}_{2} \mathrm{~F}_{3} \mathrm{Cl}_{3}$ & - & - & 187 \\
$n-\mathrm{C}_{5} \mathrm{~F}_{12}$ & 680 & 6.81 & 288 \\
\hline
\end{tabular}

Tabelle 7.3: Stoßparameter der Badgase; $\langle-\Delta E\rangle$-Werte gemessen für die Desaktivierung von Toluol mit $52000 \mathrm{~cm}^{-1}$ Schwingungsenergie [113]

den IVR hat. In diesem Fall ist die effizientere Beschleunigung des VET durch die schweren Badgase unzweifelhaft mit den größeren mittleren pro Stoß übertragenen Energien $\langle\Delta E\rangle$ dieser Stoßpartner zu korrelieren (s. Tab. 7.3). Hieraus folgt, daß der $\langle\Delta E\rangle$-Wert eines Stoßgases für die Beeinflussung der intramolekularen Dynamik eine untergeordnete Rolle spielt. Vielmehr scheint es das Stoßereignis an sich zu sein, welches die IVR-beschleunigende Störung im Benzolmolekül induziert.

Drittens ist die Steigung beider Geraden größer als Eins. Folglich ist die Beschleunigung der intramolekularen Energieumverteilung verglichen mit der des intermolekularen Energieaustauschs relativ ineffizient. Dies deutet darauf hin, daß nicht jeder Stoß, der VET bewirkt, gleichzeitig auch den IVR-Prozeß beeinflußt. Demnach reicht das Stoßereignis allein nicht aus, um die für die IVRBeschleunigung nötige Störung im Molekül zu induzieren. Es müssen anscheinend noch zusätzliche Randbedingungen, u. a. möglicherweise eine bestimmte Stoßrichtung, erfüllt sein, um die Störung an der richtigen Stelle des intramolekularen Kopplungsgefüges zu lokalisieren.

\subsubsection{Konkurrenz von Substituenten- und Umgebungseinflüssen}

Abschließend sollen an dieser Stelle die relativen Einflüsse von chemischer Konstitution und Lösungsmittelumgebung betrachtet werden. Hierfür bietet es sich an, die Ergebnisse der Gasphasenmessungen denen der IR-Pump-UV-Probe- 
7.2 IVR in unterschiedlichen molekularen Umgebungen

\begin{tabular}{|l|ccc|}
\hline & $\boldsymbol{\tau}_{\mathbf{I V R}_{\mathbf{1}}} / \mathbf{p s}$ & $\mathbf{A}_{\text {inst }}$ & $\boldsymbol{\tau}_{\mathbf{I V R}_{\mathbf{2}}} / \mathbf{p s}$ \\
\hline & \multicolumn{3}{|c}{ Gasphase } \\
Benzol & 0.5 & $0.45 \pm 0.05$ & $48 \pm 5$ \\
Toluol & 0.8 & $0.30 \pm 0.05$ & $8.2 \pm 0.8$ \\
$\alpha, \alpha, \alpha$-Trifluortoluol & 0.8 & $0.65 \pm 0.05$ & $3.8 \pm 0.5$ \\
& \multicolumn{3}{|c}{ Lösung } \\
Benzol & 0.5 & $0.49 \pm 0.06$ & $3.8 \pm 0.8$ \\
Toluol & 0.8 & $0.22 \pm 0.06$ & $4.7 \pm 0.8$ \\
$\alpha, \alpha, \alpha$-Trifluortoluol & 0.8 & $0.62 \pm 0.08$ & $3.1 \pm 0.5$ \\
\hline
\end{tabular}

Tabelle 7.4: IVR-Zeitkonstanten von Benzol, Toluol und $\alpha, \alpha, \alpha$-Trifluortoluol in der Gasphase und in 1,1,2-Trichlortrifluorethan-Lösung

Experimente an aromatischen Modellsystemen in schwach wechselwirkenden Lösungsmitteln $[13,44]$ gegenüberzustellen.

Es handelt sich hierbei um die Systeme Benzol, Toluol und $\alpha, \alpha, \alpha$-Trifluortoluol. In kondensierter Phase lagen diese Substanzen als $1 \mathrm{M}$ Lösungen in 1,1,2Trichlortrifluorethan vor. Um einen Vergleich zu ermöglichen, wurden die transienten Absorptionssignale aus der kondensierten Phase mit dem für die Gasphasendaten verwendeten kinetischen Modell neu angepaßst. In allen drei Fällen wurde - verglichen mit der monoexponentiellen IVR-Modellierung - eine Reduktion der Anpassungsresiduen erreicht. Die Zeitkonstanten der Anpassungen in Lösung sind zusammen mit den jeweiligen Gasphasenwerten in Tab. 7.4 aufgelistet.

Die Untersuchung von Benzol in überkritischem $\mathrm{CO}_{2}$ offenbarte auch bei hohen Dichten noch eine fs- und eine ps-IVR-Zeitskala, von denen lediglich letztere mit zunehmender Lösungsmitteldichte beschleunigt wurde (s. Abschn. 7.2.1). Es ist daher gerechtfertigt, die $\tau_{\mathrm{IVR}_{1}}-$ und $\tau_{\mathrm{IVR}_{2}}$-Konstanten eines Moleküls in gasförmiger und kondensierter Phase jeweils miteinander zu korrelieren.

Die Ähnlichkeit der $\tau_{\mathrm{IVR}_{1}}$ - und $A_{\text {inst }}$-Parameter desselben Moleküls impliziert, daß die ultraschnelle Komponente der Energieumverteilung nicht signifikant vom Lösungsmittel beeinflußt wird. Im Gegensatz hierzu erfährt die langsame IVRZeitskala eine ausgeprägte Kontraktion. Bemerkenswerterweise unterscheiden sich die sekundären Geschwindigkeiten in der kondensierten Phase kaum noch. Die Zeitkonstante des Toluols ist sogar größer als die des Benzols. Dies bedeutet, daß die IVR-Beschleunigung durch die Einführung des inneren Rotators, die für das 
isolierte Molekül etabliert wurde, von der Gesamtbeschleunigung der Umverteilung in der kondensierten Phase „maskiert“ wird. Derartige Effekte müssen bei der Konzeption IVR-funktioneller Gruppen für eine modenselektive Laserchemie in Lösung berücksichtigt werden. 


\section{Anhang}

\section{A.1 Eichparameter der energieabhängigen Absorption}

Bei verschiedenen Temperaturen aufgenommene UV-Spektren geben Auskunft über die Energieabhängigkeit der Absorption eines Moleküls, wenn die Temperatur auf Grundlage statistischer Thermodynamik in die Innere Energie umgerechnet wird. Aus einer Auftragung des Absorptionskoeffizienten einer Wellenlänge gegen die Innere Energie kann ein funktioneller Zusammenhang zwischen diesen Größen gewonnen werden. Die entsprechenden Auftragungen sind in den Abb. 4.2, 4.6, 4.8 und 4.10 dargestellt. Eine Anpassung der Daten bei der jeweiligen Probewellenlänge erfolgte mit Polynomen 3. Grades, die als Eichkurven in die Auswertung einbezogen wurden. Die Parameter der Polynomanpassung sind in Tab. A.1 aufgelistet. Für Benzol- $\mathrm{d}_{1}$ wurde derselbe Parametersatz verwendet wie für Benzol.

\begin{tabular}{|c|cccc|}
\hline & $\mathbf{C}_{\mathbf{6}} \mathbf{H}_{\mathbf{6}}$ & $\mathbf{C}_{\mathbf{6}} \mathbf{H}_{\mathbf{5}} \mathbf{C l}$ & $\mathbf{C}_{\mathbf{6}} \mathbf{H}_{\mathbf{5}} \mathbf{C H}_{\mathbf{3}}$ & $\mathbf{C}_{\mathbf{6}} \mathbf{H}_{\mathbf{5}} \mathbf{C F}_{\mathbf{3}}$ \\
$\boldsymbol{\lambda}_{\text {Probe }}$ & $270 \mathrm{~nm}$ & $283 \mathrm{~nm}$ & $280 \mathrm{~nm}$ & $275 \mathrm{~nm}$ \\
\hline \hline$a_{0}$ & -0.06278 & 0.11234 & 0.04468 & 0.28621 \\
$a_{1}$ & $1.87108 \cdot 10^{-4}$ & $1.0927 \cdot 10^{-4}$ & $-2.63203 \cdot 10^{-5}$ & $1.9791 \cdot 10^{-4}$ \\
$a_{2}$ & $5.01615 \cdot 10^{-8}$ & $-2.3853 \cdot 10^{-8}$ & $1.95702 \cdot 10^{-8}$ & $-3.5123 \cdot 10^{-8}$ \\
$a_{3}$ & $-4.95954 \cdot 10^{-12}$ & $2.9811 \cdot 10^{-12}$ & $-1.02268 \cdot 10^{-12}$ & $2.9498 \cdot 10^{-12}$ \\
\hline
\end{tabular}

Tabelle A.1: Anpassungsparameter der energieabhängigen Absorption 


\section{A.2 Berechnung der Zustandsdichte eines Moleküls mit internem Rotator}

Mit einem modifizierten Beyer-Swinehardt-Algorithmus [90, 91] kann die Zustandsdichte eines Moleküls mit $s$ Schwingungsfreiheitsgraden und einem internen Rotator durch direktes Auszählen ermittelt werden. Dabei wird zunächst die Zustandsdichte des freien Rotators bestimmt, welcher diskrete Energieniveaus bei

$$
E_{\mathrm{rot}}=\sigma^{2} B J^{2}
$$

besitzt. Dabei ist $B$ die Rotationskonstante des internen Rotators, $J$ die Rotationsquantenzahl und $\sigma$ die Symmetriezahl. Die $(2 J+1)$-fache Entartung der Rotationsniveaus ist in der Rechnung berücksichtigt. Die Rotationszustandsdichte wird anschließend mit der Schwingungszustandsdichte der $s$ Oszillatoren gefaltet. Die verwendeten Parameter für Toluol und $\alpha, \alpha, \alpha$-Trifluortoluol sind in Tab. A.2 zusammengestellt. Die Programmierung erfolgte in enger Anlehnung an [92].

\begin{tabular}{|l|ccc|}
\hline & $\boldsymbol{\sigma}$ & $\mathbf{B} / \mathbf{c m}^{-1}$ & $\mathbf{s}$ \\
\hline \hline Toluol & 6 & 4.87 & 38 \\
$\alpha, \alpha, \alpha$-Trifluortoluol & 6 & 3.57 & 38 \\
\hline
\end{tabular}

Tabelle A.2: Parameter der Zustandsdichtenberechnung (nach [92])

\section{A.3 Parameter der Monte Carlo-Berechnung von $\mathrm{g}(\mathrm{r})$ für Benzol- $\mathrm{CO}_{2}$}

Die Lösungsmittelparameter $\sigma_{\mathrm{v}}$ (Lennard-Jones-Durchmesser) und $\varepsilon_{\mathrm{v}}$ (Potentialtopftiefe) wurden so gewählt, daß die kritischen Daten des Lennard-Jones-Fluids,

$$
T_{\mathrm{c}}^{*}=\frac{k_{\mathrm{B}} T_{\mathrm{c}}}{\varepsilon_{\mathrm{v}}}=1.31 \quad \text { und } \quad \rho_{\mathrm{c}}^{*}=\rho_{\mathrm{c}} \sigma_{\mathrm{v}}^{3}=0.31,
$$

denen von $\mathrm{CO}_{2}$ entsprechen $\left(T_{\mathrm{c}}=304.13 \mathrm{~K}, \rho_{\mathrm{c}}=10.6 \mathrm{moll}^{-1}\right)$. Daraus ergab sich

$$
\sigma_{\mathrm{v}}=0.365 \mathrm{~nm} \quad \text { und } \quad \frac{\varepsilon_{\mathrm{v}}}{k_{\mathrm{B}}}=232.2 \mathrm{~K} \text {. }
$$

Für Benzol wurde ein effektiver Durchmesser von $\sigma_{\mathrm{u}}=0.546 \mathrm{~nm}$ angenommen. Die Topftiefe des Solvat-Solvens-Potentials wurde auf $\varepsilon / k_{\mathrm{B}}=500 \mathrm{~K}$ angepaßt. 


\section{Abbildungsverzeichnis}

2.1 Graphische Darstellung des IVR-Stufenmodells . . . . . . . . . . . 13

2.2 Schematische Darstellung eines dreidimensionalen QNS . . . . . 15

3.1 Prinzip der IR-Pump-UV-Probe-Spektroskopie . . . . . . . . . . 28

3.2 Typisches transientes Absorptionssignal . . . . . . . . . . . . . . . . . . . 30

3.3 Prinzipieller Aufbau des Lasersystems . . . . . . . . . . . . . . . . 31

3.4 Optischer Aufbau des Hurricane ${ }^{\mathrm{TM}}-$ Systems $\ldots . . . . . .33$

3.5 Optischer Aufbau des Streckers und des Kompressors . . . . . . . 36

3.6 Optischer Aufbau des regenerativen Verstärkers (RA) . . . . . . . 37

3.7 Spektrum und Autokorrelation des Hurricane ${ }^{\mathrm{TM}} \ldots \ldots . . \quad \ldots 9$

3.8 Optischer Aufbau des NOPA-Systems . . . . . . . . . . . . . . . . 42

3.9 Spektren und Autokorrelation des NOPA-Systems . . . . . . . . . 44

3.10 Spektrum und Autokorrelation des TOPAS-Systems . . . . . . . . 47

3.11 Optischer Aufbau des Pump-Probe-Spektrometers . . . . . . . . . 49

3.12 Kreuzkorrelationen von Pump- und Probepuls . . . . . . . . . . . 53

3.13 Schematischer Aufbau der Hochdruck-/Vakuumapparatur . . . . . 56

4.1 nIR-Absorptionsspektrum des Benzols . . . . . . . . . . . 65

4.2 UV-Absorptionsspektren und Energieabhängigkeit der Absorption von Benzol . . . . . . . . . . . . . . . . . . . . 68

4.3 nIR-Absorptionsspektrum des Monodeutero-Benzols . . . . . . . 71

4.4 UV-Absorptionsspektrum des verdünnten Monodeuterobenzols . . 73

4.5 nIR-Absorptionsspektrum des Chlorbenzols . . . . . . . . . 76

4.6 UV-Absorptionsspektren und Energieabhängigkeit der Absorption des Chlorbenzols . . . . . . . . . . . . . . . . 78

4.7 nIR-Absorptionsspektrum des Toluols . . . . . . . . . . . . 82 
4.8 UV-Absorptionsspektren und Energieabhängigkeit der Absorption des Toluols . . . . . . . . . . . . . . . . . . . . . . 84

4.9 nIR-Absorptionsspektrum des $\alpha, \alpha, \alpha$-Trifluortoluols . . . . . . . . 87

4.10 UV-Absorptionsspektren und Energieabhängigkeit der Absorption des $\alpha, \alpha, \alpha$-Trifluortoluols . . . . . . . . . . . . . . . . . 89

4.11 Energieniveau-Diagramm der Fundamentalschwingungen der fünf Modellsysteme . . . . . . . . . . . . . . . . . . . . 90

4.12 Vergleich der Zustandsdichten der fünf Modellsysteme . . . . . . . 91

5.1 Hochaufgelöste transiente Absorption des reinen gasförmigen Benzols 95

5.2 Transiente Absorption des reinen gasförmigen Benzols bei verschiedenen Anregungsenergien . . . . . . . . . . . . . . . . . . . . . . . 96

5.3 Transiente Absorption des reinen gasförmigen Benzols bei verschiedenen Temperaturen I . . . . . . . . . . . . . . . . . . . . . . . . 98

5.4 Transiente Absorption des reinen gasförmigen Benzols bei verschiedenen Temperaturen II . . . . . . . . . . . . . . . . . . . . . . 99

5.5 Transiente Absorption des reinen gasförmigen Benzols bei verschiedenen Benzoldrücken I . . . . . . . . . . . . . . . . . . . . . . 101

5.6 Transiente Absorption des reinen gasförmigen Benzols bei verschiedenen Benzoldrücken II . . . . . . . . . . . . . . . . . . . . . . . 102

5.7 Transiente Absorption des reinen gasförmigen Benzol- $\mathrm{d}_{1}$ bei verschiedenen Anregungsenergien . . . . . . . . . . . . . . . . . . . . 104

5.8 Vergleich der transienten Absorptionen von Benzol und Benzol- $\mathrm{d}_{1} 105$

5.9 Transiente Absorption des reinen gasförmigen Chlorbenzols . . . . 107

5.10 Transiente Absorption des reinen gasförmigen Toluols . . . . . . . 108

5.11 Transiente Absorption des reinen gasförmigen $\alpha, \alpha, \alpha$-Trifluortoluols 109

5.12 Vergleich der transienten Absorptionen der fünf aromatischen Modellsysteme . . . . . . . . . . . . . . . . . . 111

5.13 Transiente Absorption von Benzol in Helium, Argon und 1,1,2-Trichlortrifluorethan . . . . . . . . . . . . . . . 114

5.14 Transiente Absorption von Benzol in $\mathrm{CO}_{2}$ bei verschiedenen Badgasdrücken . . . . . . . . . . . . . . . . . . . . 116

5.15 Transiente Absorption von Benzol in $\mathrm{C}_{5} \mathrm{~F}_{12} \ldots \ldots . . . .118$

5.16 Vergleich der transienten Absorptionen von Benzol in verschiedenen molekularen Umgebungen . . . . . . . . . . . . . . . . . . . 119 
Abbildungsverzeichnis

5.17 QNS-Simulation für Kombinationstonanregung $\left(1 \nu_{4}+1 \nu_{7}\right)$ in Ben-

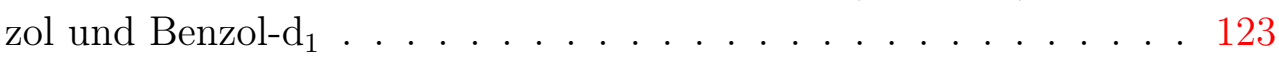

5.18 Effekt der Anregungsenergiebreite für Benzol und Benzol- $\mathrm{d}_{1}$. . . 125

5.19 Effekt der isotropen QNS-Verdünnung für Benzol und Benzol-d $\mathrm{d}_{1}$. 126

5.20 QNS-Simulation für Benzol und Benzol-d $\mathrm{d}_{1}$ bei $6000 \mathrm{bzw} .4500 \mathrm{~cm}^{-1}$ Anregungsenergie . . . . . . . . . . . . . . . . . . . . 128

5.21 QNS-Simulation für Benzol und Benzol-d $\mathrm{d}_{1}$ bei 3000 bzw. $4500 \mathrm{~cm}^{-1}$ Anregungsenergie . . . . . . . . . . . . . . . . . . . . . . . . . 129

5.22 QNS-Simulation für Benzol nach $1 \nu_{2}$-Anregung, Vergleich mit Lösungsexperiment von Dlott et al. . . . . . . . . . . . . . . . . 132

6.1 Anpassung der transienten Absorption von Benzol I . . . . . . . . 143

6.2 Anpassung der transienten Absorption von Benzol II . . . . . . . 144

6.3 Anpassung der transienten Absorption von Benzol III . . . . . . . 145

6.4 Anpassung der transienten Absorption von Benzol- $\mathrm{d}_{1}$. . . . . . . 146

6.5 Anpassung der transienten Absorption von Chlorbenzol, Toluol und $\alpha, \alpha, \alpha$-Trifluortoluol . . . . . . . . . . . . . . . . . . . 147

6.6 Anpassung der transienten Absorption von Benzol in Helium, Argon und 1,1,2-Trichlortrifluorethan . . . . . . . . . . . . . 151

6.7 Anpassung der transienten Absorption von Benzol in $\mathrm{CO}_{2}$. . . . 152

6.8 Anpassung der transienten Absorption von Benzol in $\mathrm{C}_{5} \mathrm{~F}_{12}$. . . 153

7.1 Quantendynamische Simulation von Wyatt et al. . . . . . . 158

7.2 Temperaturabhängigkeit von $\tau_{\mathrm{IVR}_{2}}$ und $\mathrm{A}_{\text {inst }}$ des Benzols . . . . . 161

7.3 Zeitaufgelöste Besetzungsdiffusion im QNS des Benzols . . . . . . 167

7.4 Zeitaufgelöste Besetzungsdiffusion im QNS des Benzol-d $\mathrm{d}_{1}$. . . . . 168

7.5 Statistische Besetzung der Normalmoden des Benzols nach 1 bzw. $10 \mathrm{ps}$ Simulationszeit . . . . . . . . . . . . . . 170

7.6 Statistische Besetzung der Normalmoden des Benzol- $\mathrm{d}_{1}$ nach 1 bzw. 10 ps Simulationszeit . . . . . . . . . . . . . . . . 171

7.7 Relative Beschleunigung der langsamen IVR-Komponente durch chemische Substitution . . . . . . . . . . . . . . . . . 178

7.8 Korrelation zwischen IVR-Geschwindigkeit und nach Kopplungsordnung sortierter Zustandsdichte für 30-Moden-Aromaten . . . . 180

7.9 Dichteabhängigkeit der Geschwindigkeitskonstanten von IVR,2 und VET in überkritischem $\mathrm{CO}_{2}$. . . . . . . . . . . . . . 182 
7.10 Radiale Dichteverteilungen um ein attraktives Lennard-JonesTeilchen in einem Lennard-Jones-Fluid . . . . . . . . . . . . . . 183

7.11 Korrelation der Geschwindigkeitskonstanten von IVR,2 und VET mit der lokalen $\mathrm{CO}_{2}$-Dichte . . . . . . . . . . . . . . . . 185

7.12 Korrelation der relativen Beschleunigungen von IVR,2 und VET in verschiedenen Inertgasumgebungen . . . . . . . . . . . . . 186 


\section{Tabellenverzeichnis}

4.1 Die 30 Normalschwingungen des Benzols . . . . . . . . . . . . . 62

4.2 Die 30 Normalschwingungen des Benzol- $\mathrm{d}_{1} \ldots \ldots$. . . . . . . 70

4.3 Die 30 Normalschwingungen des Chlorbenzols . . . . . . . . . . . . 75

4.4 Die 38 Normalschwingungen des Toluols . . . . . . . . . . . . . 80

4.5 Die 38 Normalschwingungen des $\alpha, \alpha, \alpha$-Trifluortoluols . . . . . . . 86

6.1 Zeitkonstanten für die Anpassungen der Messungen an Benzol bei verschiedenen Anregungswellenlängen . . . . . . . . . . . . . . . 148

6.2 Zeitkonstanten für die Anpassungen der Messungen an Benzol bei verschiedenen Meßstemperaturen . . . . . . . . . . . . . . 148

6.3 Zeitkonstanten für die Anpassungen der Messungen an Benzol bei verschiedenen Benzoldrücken . . . . . . . . . . . . . . . . . . . 148

6.4 Zeitkonstanten für die Anpassungen der Messungen an Benzol- $\mathrm{d}_{1}$, Chlorbenzol, Toluol und $\alpha, \alpha, \alpha$-Trifluortoluol . . . . . . . . . . 149

6.5 Zeitkonstanten für die Anpassungen der Messungen an Benzol in verschiedenen Badgasen . . . . . . . . . . . . . . . . 154

6.6 Zeitkonstanten für die Anpassungen der Messungen an Benzol in überkritischem $\mathrm{CO}_{2} \ldots \ldots \ldots \ldots$. . . . . . . . . . . 154

6.7 Zeitkonstanten für die Anpassungen der Messungen an Benzol in überkritischem $\mathrm{C}_{5} \mathrm{~F}_{12} \ldots \ldots \ldots \ldots \ldots$

7.1 Symmetriereduzierte Zustandsdichten von Benzol und Benzol- $\mathrm{d}_{1}$. 163

7.2 Nach Kopplungsordnung sortierte Zustandsdichte für die $2 v_{\mathrm{CH}-\text { streck}}$-Anregung in 30-Moden-Aromaten . . . . . . . . . . 179

7.3 Stoßparameter der Badgase . . . . . . . . . . . . . . . . 188

7.4 IVR-Zeitkonstanten von Benzol, Toluol und $\alpha, \alpha, \alpha$-Trifluortoluol in der Gasphase und in 1,1,2-Trichlortrifluorethan-Lösung . . . . . 189 
Tabellenverzeichnis

A.1 Anpassungsparameter der energieabhängigen Absorption . . . . . 191

A.2 Parameter der Zustandsdichtenberechnung . . . . . . . . . . . . . 192 


\section{Literaturverzeichnis}

[1] D. J. Nesbitt, R. W. Field, J. Phys. Chem. A 1996, 100, 12735. 3

[2] M. Gruebele, R. Bigwood, Int. Rev. Phys. Chem. 1998, 17, 91.3

[3] T. F. Deutsch, S. R. J. Brueck, Chem. Phys. Lett. 1977, 54, 258. 4

[4] J. C. Deak, Y. Pang, T. D. Sechler, Z. Wang, D. D. Dlott, Science 2004, 306, 473. 4

[5] J.-Y. Liu, W.-H. Fan, K.-L. Han, D.-L. Xu, N.-Q. Lou, J. Phys. Chem. A 2003, 107, 1914. 4, 162

[6] D. Schwarzer, P. Kutne, J. Schröder, J. Troe, J. Chem. Phys. 2004, 121, 1754. 4

[7] S. A. Schofield, G. Wolynes, J. Chem. Phys. 1993, 98, 1123. 5

[8] D. M. Leitner, P. G. Wolynes, J. Chem. Phys. 1996, 105, 11226. 5

[9] D. M. Leitner, P. G. Wolynes, J. Phys. Chem. A 1997, 101, 541. 5

[10] M. Bixon, J. Jortner, J. Chem. Phys. 1968, 48, 715. 12, 17

[11] A. A. Stuchebrukhov, R. A. Marcus, J. Chem. Phys. 1993, 98, 6044. 13

[12] M. Gruebele, Adv. Chem. Phys. 2001, 114, 193. 14

[13] J. Aßmann, Untersuchungen des zeitaufgelösten Schwingungsenergietransfers organischer Moleküle in der kondensierten Phase, Dissertation, Universität Göttingen, 2002. 17, 24, 27, 52, 60, 61, 122, 139, 189

[14] C. S. Parmenter, Disc. Faraday Chem. Soc. 1983, 75, 7. 18 
[15] C. S. Parmenter, B. M. Stone, J. Chem. Phys. 1986, 84, 4710. 22, 176

[16] D. B. Moss, C. S. Parmenter, G. E. Ewing, J. Chem. Phys. 1987, 86, 51. $22,175,176$

[17] Z.-Q. Zhao, C. S. Parmenter, D. B. Moss, A. J. Bradley, A. E. W. Knight, K. G. Owens, J. Chem. Phys. 1992, 96, 6362. 22

[18] D. B. Moss, C. S. Parmenter, J. Chem. Phys. 1993, 98, 6897. 22

[19] A. McIlroy, D. J. Nesbitt, J. Chem. Phys. 1994, 101, 3421. 22

[20] J. E. Gambogi, R. Z. Pearson, X. Yang, K. K. Lehmann, G. Scoles, J. Chem. Phys. 1995, 190, 191. 22

[21] D. S. Perry, J. Chem. Phys. 1993, 98, 6665. 22

[22] A. McIlroy, D. J. Nesbitt, J. Chem. Phys. 1990, 92, 2229. 22

[23] G. T. Fraser, B. H. Pate, G. A. Bethardy, D. S. Perry, J. Chem. Phys. 1993, 175, 223. 22

[24] G. A. Bethardy, X. Wang, D. S. Perry, Can. J. Chem. 1994, 72, 652. 22

[25] K. K. Lehmann, G. Scoles, B. H. Pate, Ann. Rev. Phys. Chem. 1994, 45, 241. 22,173

[26] J. E. Gambogi, R. P. L'Esperance, K. K. Lehmann, B. H. Pate, G. Scoles, J. Chem. Phys. 1993, 98, 1112. 22, 173

[27] L. D. Landau, E. A. Teller, Phys. Z. Sowj. Un. 1936, 10, 34. 23

[28] R. N. Schwartz, Z. I. Slawsky, K. F. Herzfeld, J. Chem. Phys. 1952, 20, 1591. 23

[29] C. J. Pursell, C. S. Parmenter, J. Phys. Chem. 1993, 97, 1615. 23

[30] J. Catlett, D. L., C. S. Parmenter, C. J. Pursell, J. Chem. Phys. 1994, 98, 3263. 23 
[31] T. Lenzer, Experimentelle und theoretische Untersuchungen zur Stoßrelaxation hoch schwingungsangeregter Benzolderivate im Bereich hoher Zustandsdichten, Dissertation, Universität Göttingen, 1995. 23

[32] R. Zwanzig, J. Chem. Phys. 1961, 34, 1931. 23, 24

[33] D. Schwarzer, J. Troe, M. Votsmeier, M. Zerezke, J. Chem. Phys. 1996, 105, 3121. 23

[34] D. Schwarzer, J. Troe, M. Zerezke, J. Chem. Phys. 1997, 107, 8380. 23, 61,183

[35] D. Schwarzer, J. Troe, M. Zerezke, J. Chem. Phys. 1998, 102, 4207. 23, 183

[36] C. Heidelbach, Computersimulationen zum Schwingungsenergietransfer in verdichteter, fluider Phase, Dissertation, Universität Göttingen, 1998. 24

[37] J. C. Owrustky, D. Raftery, R. M. Hochstrasser, Ann. Rev. Phys. Chem. 1994, 45, 519. 24

[38] S. A. Egorov, J. L. Skinner, J. Chem. Phys. 1996, 105, 7047. 24

[39] D. W. Oxtoby, Adv. Chem. Phys. 1981, 47, 487. 24

[40] R. M. Stratt, Acc. Chem. Res. 1995, 28, 201. 24

[41] G. Goodyear, R. M. Stratt, J. Chem. Phys. 1996, 105, 10050. 24

[42] J. Jang, R. M. Stratt, J. Chem. Phys. 2000, 112, 7524. 24

[43] O. Link, Zeitaufgelöste Untersuchungen des Schwingungsenergietransfers von aromatischen Molekülen in Lösung und überkritischem $\mathrm{CO}_{2}$, Diplomarbeit, Universität Göttingen, 2003. 27, 52, 60, 61, 113, 117

[44] R. von Benten, Zeitaufgelöste Untersuchungen des Schwingungsenergietransfers von aromatischen Molekülen in Lösung, Diplomarbeit, Universität Göttingen, 2002. 27, 45, 52, 60, 61, 117, 138, 139, 189

[45] D. Bingemann, M. P. Gorman, A. W. King, F. F. Crim, J. Chem. Phys. 1997, 10\%, 661. 27 
[46] D. Bingemann, A. W. King, F. F. Crim, J. Chem. Phys. 2000, 113, 5018. 27

[47] G. Herzberg, Molecular Spectra and Molecular Structure, Vol. 3: Electronic spectra and electronic structure of polyatomic molecules (Van Nostrand Reinhold), 1966. 29

[48] J. M. Hollas, High Resolution Spectroscopy (John Wiley and Sons), 1998. 29

[49] Spectra Physics Lasers, Mountain View, CA, USA, Hurricane, One-box, diode-pumped, kilohertz Ti:Sapphire regenerative amplifier system, User Manual, 1.00 Aufl., 2000. 32

[50] Spectra Physics Lasers, Mountain View, CA, USA, Mai Tai, Diode-pumped, mode-locked Ti:sapphire Laser, User's Manual, 2002. 32

[51] Spectra Physics Lasers, Mountain View, CA, USA, Evolution, Multikilohertz, intra-cavity doubled, diode-pumped Nd:YLF Laser, User Manual, Version 2.2, 2001. 32

[52] G. Gale, T. Cavallari, T. J. Driscoll, F. Hache, Optics Letters 1995, 20, 1562. 41

[53] Y. R. Shen, The principles of Nonlinear Optics (Wiley \& Sons), 1984. 42

[54] R. Laenen, H. Graener, A. Laubereau, J. Opt. Soc. Am. B 1991, 8, 1085. 46

[55] G. Mak, Q. Fu, H. M. van Driel, Appl. Phys. Lett. 1992, 60, 542.46

[56] A. Charvat, J. Aßmann, D. Schwarzer, K. Henning, K. Luther, B. Abel, J. Troe, PCCP 2001, 3, 2230. 61

[57] L. Brouwer, H. Hippler, L. Lindemann, J. Troe, J. Phys. Chem. 1985, 89, 4608. 61

[58] A. Callegari, U. Merker, P. Engels, H. K. Srivastava, K. K. Lehmann, G. Scoles, J. Chem. Phys. 2000, 113, 10583. 62, 157 
[59] E. B. Wilson jr., Phys. Rev. 1934, 45, 706. 62, 63

[60] G. Varsanyi, in Assignment for Vibrational Spectra of 700 Benzene Derivates (Akademiai Kaido, Budapest), 1973 . 63, 85

[61] W. Roth, Varsanyi's modes of benzene, http://home.arcor.de/rothw/gauss/varsanyi/molekuele/Bz/, 1999. 63

[62] M. Quack, Ann. Rev. Phys. Chem. 1990, 41, 839. 63

[63] F. Iachello, S. Oss, J. Chem. Phys. 1993, 99, 7337. 64

[64] R. H. Page, Y. R. Shen, Y. T. Lee, J. Chem. Phys. 1987, 88, 4621. 65, 156,157

[65] E. L. I. Sibert, W. P. Reinhardt, J. T. Hynes, J. Chem. Phys. 1984, 81, 1115. 65

[66] D. L. Snavely, V. A. Walters, S. D. Colson, K. B. Wiberg, Chem. Phys. Lett. 1984, 103, 423. 65

[67] F. Turkevich, M. Fred, Rev. Mod. Phys. 1942, 14, 246. 66

[68] J. H. Callomon, T. M. Dunn, I. M. Mills, Phil. Trans. Roy. Soc. Lond. 1966, A259, 499. 66, 72, 121

[69] A. Y. Bertie, J. E., C. D. Keefe, J. Mol. Struct. 2000, 550-551, 135. 69, 70,72

[70] E. R. Bernstein, J. Chem. Phys. 1970, 52, 4701. 70

[71] E. Riedle, A. Beil, D. Luckhaus, M. Quack, Molecular Physics 1994, 81, 1. 73

[72] Y. S. Jain, H. D. Bist, J. Mol. Spec. 1973, 47, 126. 75

[73] K. M. Gough, B. R. Henry, J. Phys. Chem. 1984, 87, 3433. 76

[74] I. A. Watson, B. R. Henry, Spectrochim. Acta 1981, A37, 857. 76 
[75] O. S. Mortensen, B. R. Henry, M. A. Mohammadi, J. Chem. Phys. 1981, $75,4800.76$

[76] J. Petruska, J. Chem. Phys. 1961, 34, 1120. 77

[77] A. M. Bass, H. Sponer, J. Opt. Soc. Am. 1950, 40, 389. 77

[78] H. Rudolph, J. Dreizler, J. Jaeschke, P. Wendling, Z. Naturforschung A 1967, 22, 940. 79

[79] W. A. Kreiner, H. D. Rudolph, B. Tan, J. Mol. Spec. 1973, 48, 86. 79

[80] N. Fuson, C. Garrigou-Lagrange, M. L. Josien, Spectrochim. Acta 1960, 16, 106. 79,80

[81] H. G. Kjaergaard, D. M. Turnbull, B. R. Henry, J. Phys. Chem. A 1998, 102, 6095. 81

[82] H. G. Kjaergaard, D. M. Turnbull, B. R. Henry, J. Phys. Chem. A 1997, 101, 2589. 81

[83] H. G. Kjaergaard, Z. Rong, A. J. McAlees, D. L. Howard, B. R. Henry, J. Phys. Chem. A 2000, 104, 6398. 81

[84] P. N. Gosh, P. K. Panja, C. M. Pal, Chem. Phys. Lett. 1988, 148, 337. 81

[85] K. M. Gough, B. R. Henry, J. Phys. Chem. 1984, 88, 1298. 81

[86] A. M. Bass, J. Chem. Phys. 1950, 18, 1403. 82

[87] T. Ogata, A. P. Cox, J. Mol. Spec. 1976, 61, 265. 85

[88] D. W. Scott, D. R. Douslin, J. F. Messrly, S. S. Todd, I. A. Hossenlopp, T. C. Knicheloe, J. P. McCullogh, J. Am. Chem. Soc. 1959, 81, 1015. 85, 86

[89] M. L. N. Sastri, H. Sponer, J. Chem. Phys. 1952, 20, 1428. 87

[90] T. Beyer, D. F. Swinehardt, Comm. Assoc. Comput. Machines 1973, 16, 379. 92,192

[91] S. E. Stein, B. S. Rabinovitch, J. Chem. Phys. 1973, 58, 2438. 92, 192 
[92] T. Lenzer, A. C. Symonds, A program for calculating the rovibrational density of states for Toluene, V1.0, University of Göttingen, 1995. 92, 192

[93] J. Aßmann, R. von Benten, A. Charvat, B. Abel, J. Phys. Chem. A 2003, 107, 5291. 112, 150

[94] J. Aßmann, R. von Benten, A. Charvat, B. Abel, J. Phys. Chem. A 2003, 107, 1904. 112, 131, 133, 140, 150

[95] J. Aßmann, A. Charvat, D. Schwarzer, C. Kappel, K. Luther, B. Abel, J. Phys. Chem. 2001. 112, 150

[96] R. von Benten, A. Charvat, O. Link, B. Abel, D. Schwarzer, Chem. Phys. Lett. 2004, 386, 325. 113, 117, 184, 187

[97] R. von Benten, O. Link, B. Abel, D. Schwarzer, J. Phys. Chem. A 2004, 108, 363. 117,174

[98] G. M. Stewart, J. D. McDonald, J. Chem. Phys. 1983, 78, 3907. 130, 133

[99] L. K. Iwaki, J. C. Deak, S. T. Rhea, D. D. Dlott, Chem. Phys. Lett. 1999, 303, 176. 130, 132

[100] M. Hippler, R. Pfab, M. Quack, J. Phys. Chem. A 2003, 107, 10743. 157, 173

[101] J. A. Nicholson, W. D. Lawrance, Chem. Phys. Lett. 1995, 236, 336. 157

[102] T. J. Minehardt, J. D. Adcock, R. E. Wyatt, Chem. Phys. Lett. 1999, 303, 537. 157,158

[103] Y. F. Zhang, R. A. Marcus, J. Chem. Phys. 1992, 96, 6065. 157

[104] Y. F. Zhang, R. A. Marcus, J. Chem. Phys. 1992, 97, 5283. 157

[105] T. J. Minehardt, J. D. Adcock, R. E. Wyatt, J. Chem. Phys. 1999, 110, 3326. 157

[106] H. S. Yoo, D. A. McWhorter, B. H. Pate, J. Phys. Chem. A 2004, 108, 1380. 161 
[107] L. W. Peng, B. W. Keelan, D. H. Semmes, A. H. Zewail, J. Phys. Chem. 1988, 92, 5540. 173

[108] C. C. Martens, W. P. Reinhardt, J. Chem. Phys. 1990, 93, 5621. 175, 176, 177

[109] Y. Guan, D. L. Thompson, J. Chem. Phys. 1990, 92, 313. 176

[110] D. R. Borst, D. W. Pratt, J. Chem. Phys. 2000, 113, 3658. 176

[111] J. E. Gambogi, K. K. Lehmann, B. H. Pate, G. Scoles, X. Yang, J. Chem. Phys. 1993, 98, 1748. 179

[112] J. Aßmann, M. Kling, B. Abel, Angew. Chem. Int. Ed. 2003, 42, 2226. 179

[113] H. Hippler, J. Troe, H. J. Wendelken, J. Chem. Phys. 1983, 78, 6709. 188 


\section{Lebenslauf}

Am 1. Juni 1978 wurde ich, Rebekka Susanne von Benten, als Tochter der Eheleute Dr. Charlotte Elisabeth von Benten, geb. Brandt, und Dr. Klaus von Benten in Braunschweig geboren. Dort lebte ich bis 1997. Von 1984 bis 1988 besuchte ich die Grundschule „Pestalozzistraße“. Nach dem Besuch der Orientierungsstufe „Diesterweg“ wechselte ich 1990 auf das Gymnasium Martino-Katharineum, an dem ich im Juni 1997 das Zeugnis der Allgemeinen Hochschulreife erwarb.

Zum Wintersemester 1997/98 nahm ich an der Georg-August-Universität zu Göttingen den Studiengang Humanmedizin auf. Zum Sommersemester 1998 wechselte ich an dieser Universität zum Diplomstudiengang Chemie. Die DiplomVorprüfung legte ich im April 2000 ab. Nach einem dreimonatigen Forschungspraktikum im „Arrhenius Laboratoriet“ der Stockholms Universitet bei Prof. Dr. Jan Bäckvall in der Zeit vom April 2001 bis Juli 2001 begann ich im Oktober 2001 unter der Leitung von Prof. Dr. Jürgen Troe am Institut für Physikalische Chemie der Universität Göttingen meine Diplomarbeit zum Thema „Zeitaufgelöste Untersuchungen des Schwingungsenergietransfers von aromatischen Molekülen in Lösung“" Im Juli 2002 absolvierte ich das Diplom-Hauptexamen.

In den folgenden drei Jahren erforschte ich als Stipendiatin des Graduiertenkollegs 782 „Spektroskopie und Dynamik molekularer Knäuel und Aggregate“ den Schwingungsenergietransfer aromatischer Moleküle in verschiedenen molekularen Umgebungen. Als Ergebnis dieser Arbeit entstand die vorliegende Dissertationsschrift. 